\title{
Population Dose Commitments Due to Radioactive Releases from Nuclear Power Plant Sites in 1988
}

Manuscript Completed: January 1992

Date Published: January 1992

Prepared by

D. A. Baker

Pacific Northwest Laboratory

Richland, WA 99352

\section{Prepared for}

Division of Computer and Telecommunications Services

Office of Information Resources Management

U.S. Nuclear Regulatory Commission

Washington, DC 20555

NRC FIN B2243 


\section{DISCLAIMER}

This report was prepared as an account of work sponsored by an agency of the United States Government. Neither the United States Government nor any agency Thereof, nor any of their employees, makes any warranty, express or implied, or assumes any legal liability or responsibility for the accuracy, completeness, or usefulness of any information, apparatus, product, or process disclosed, or represents that its use would not infringe privately owned rights. Reference herein to any specific commercial product, process, or service by trade name, trademark, manufacturer, or otherwise does not necessarily constitute or imply its endorsement, recommendation, or favoring by the United States Government or any agency thereof. The views and opinions of authors expressed herein do not necessarily state or reflect those of the United States Government or any agency thereof. 


\section{DISCLAIMER}

Portions of this document may be illegible in electronic image products. Images are produced from the best available original document. 
PREVIOUS REPORTS IN THIS SERIES

1. Population Dose Commitments Due to Radioactive Releases from Nuclear Power Plant Sites in 1975, PNL-2439, October 1977.

2. Population Dose Commitments Due to Radioactive Releases from Nuclear. Power Plant Sites in 1976, NUREG/CR-1125, PNL-2940, December 1979.

3. Population Dose Commitments Due to Radioactive Releases from Nuclear Power Plant Sites in 1977, NUREG/CR-1498, PNL-3324, October 1980.

4. Population Dose Commitments Due to Radioactive Releases from Nuclear Power Plant Sites in 1978, NUREG/CR-2201, PNL-4039, June 1982.

5. Population Dose Commitments Due to Radioactive Releases from Nuclear Power Plant Sites in 1979, NUREG/CR-2850, PNL-4221, Vol. 1, December 1982.

6. Population Dose Commitments Due to Radioactive Releases from Nuclear Power Plant Sites in 1980, NUREG/CR-2850, PNL-4221, Vol. 2, August 1983.

7. Population Dose Commitments Due to Radioactive Releases from Nuclear Power Plant Sites in 1981, NUREG/CR-2850, PNL-4221, Vo1. 3, January 1985.

8. Population Dose Commitments Due to Radioactive Releases from Nuclear Power Plant Sites in 1982, NUREG/CR-2850, PNL-4221, Vol. 4, June 1986.

9. Population Dose Commitments Due to Radioactive Releases from Nuclear Power Plant Sites in 1983, NUREG/CR-2850, PNL-4221, Vol. 5, January 1987.

10. Population Dose Commitments Due to Radioactive Releases from Nuclear Power Plant Sites in 1984, NUREG/CR-2850, PNL-4221, Vol. 6, January 1988.

11. Population Dose Commitments Due to Radioactive Releases from Nuclear Power Plant Sites in 1985, NUREG/CR-2850, PNL-4221, Vo1. 7, August 1988.

12. Population Dose Commitments Due to Radioactive Releases from Nuclear Power Plant Sites in 1986, NUREG/CR-2850, PNL-4221, Vo1. 8, September 1989

13. Population Dose Commitments Due to Radioactive Releases from Nuclear Power Plant Sites in 1987, NUREG/CR-2850, PNL-4221; Vol. 9, August 1990 


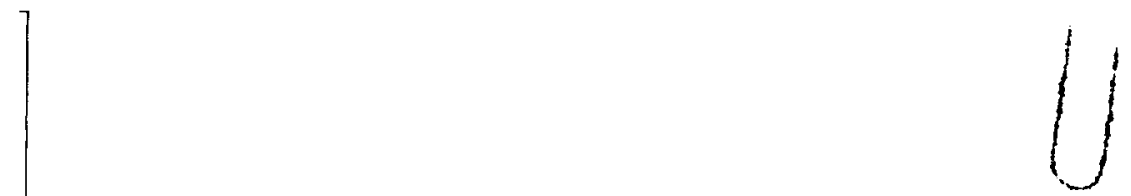

\section{ABSTRACT}

Population radiation dose commitments have been estimated from reported radionuclide releases from commercial power reactors operating during 1988. Fifty-year dose commitments for a one-year exposure from both liquid and atmospheric releases were calculated for four population groups (infant, child, teen-ager and adult) residing between 2 and $80 \mathrm{~km}$ from each of 71 reactor sites. This report tabulates the results of these calculations, showing the dose commitments for both water and airborne pathways for each age group and organ. Also included for each of the sites is a histogram showing the fraction of the total population within 2 to 80 $\mathrm{km}$ around each site receiving various average dose commitments from the airborne pathways.

The total collective dose commitments (from both liquid and airborne pathways) for each site ranged from a high of 16 person-rem to a low of 0.0011 person-rem for the sites with plants operating throughout the year with an arithmetic mean of 1.1 person-rem. The total population dose for all sites was estimated at 75 person-rem for the 150 million people considered at risk.

The site average individual dose commitment from all pathways ranged from a low of $3 \times 10^{-7}$ mrem to a high of $0.02 \mathrm{mrem}$. No attempt was made in this study to determine the maximum dose commitment received by any one individual from the radionuclides released at any of the sites. However, licensee calculation of doses to the maximally exposed individual at some sites indicated values of up to approximately 100 times average individual doses (on the order of a few millirem per year). 


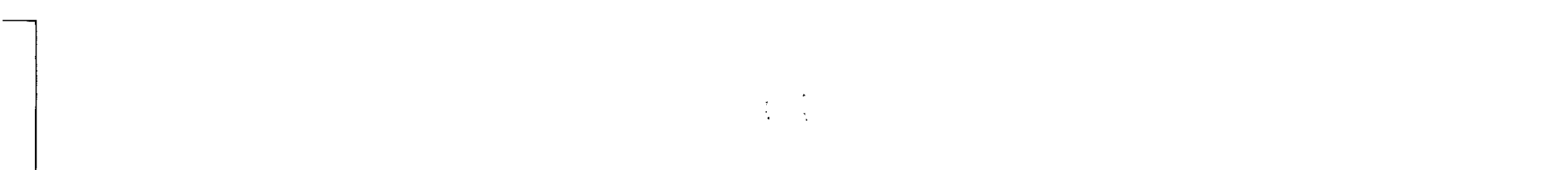

$$
\text { : }
$$

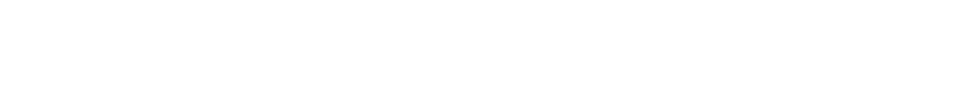

.




\section{CONTENTS}

ABSTRACT ...........................

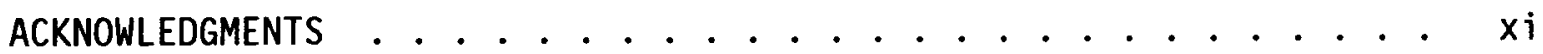

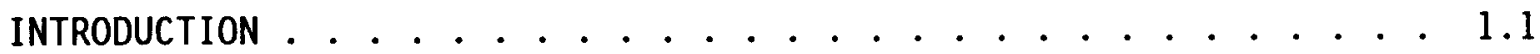

Site-Dependent Parameters . . . . . . . . . . 1.6

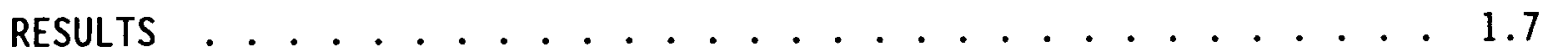

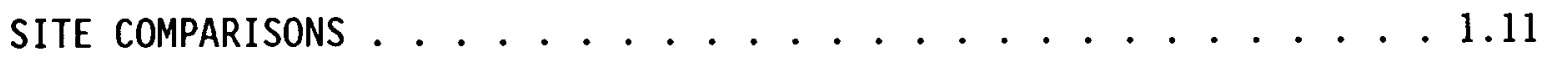

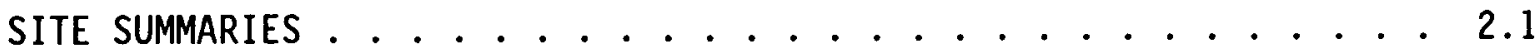

Arkansas 0 e $1 \& 2 \ldots \ldots \ldots . \ldots . \ldots . \ldots 2$

Beaver Valley $1 \& 2 \ldots \ldots . \ldots . \ldots . \ldots$

Big Rock Point . . . . . . . . . . . 2.6

Braidwood $1 \& 2 \ldots \ldots \ldots \ldots$

Browns Ferry 1, 2, \& $3 \ldots \ldots . \ldots \ldots$

Brunswick $1 \& 2 \ldots \ldots . \ldots \ldots . \ldots \ldots$

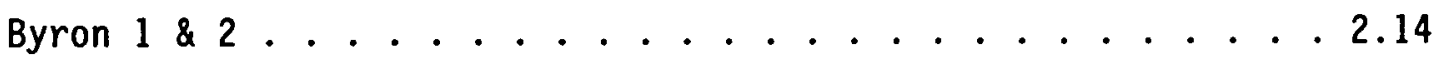

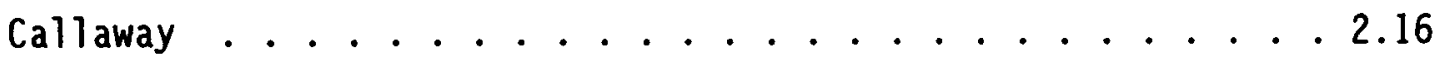

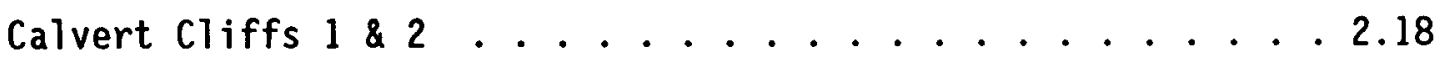

Catawba $1 \& 2 \ldots \ldots . \ldots \ldots$

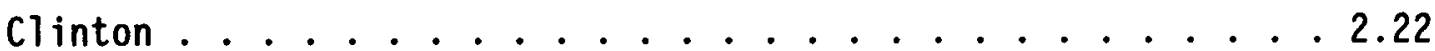

Cook $1 \& 2 \ldots \ldots \ldots \ldots \ldots . \ldots \ldots$

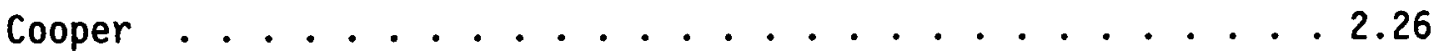

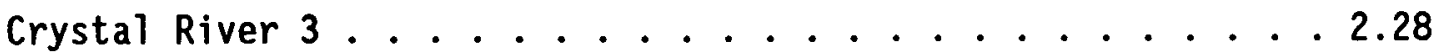

Davis-Besse . . . . . . . . . . . . 2.30

Diablo Canyon $1 \& 2 \ldots \ldots . \ldots . \ldots . \ldots . \ldots . \ldots$ 
Dresden $1,2, \& 3 \ldots \ldots \ldots \ldots \ldots$

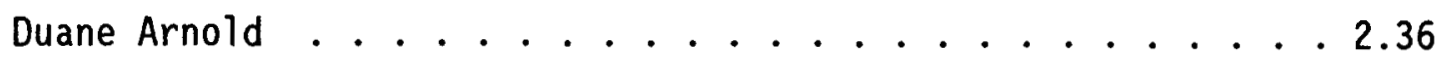

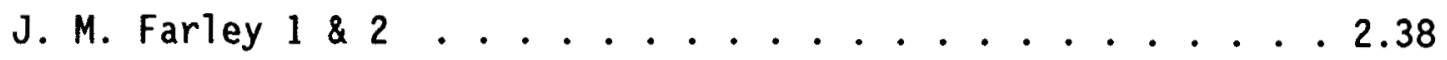

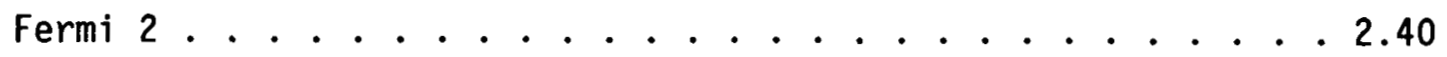

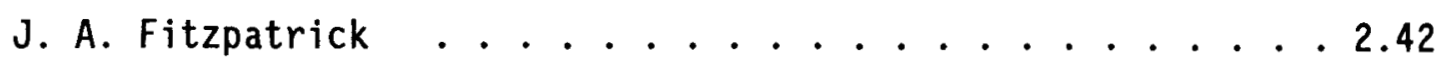
Fort Calhoun .......................... 2.44 R. E. Ginna ......................... 2.46 Grand Gulf . . . . . . . . . . . . . . 2.48 Haddam Neck . . . . . . . . . . . . . . 2.50 Harris ................... . . 2.52 Edwin I. Hatch ......................... 2.54

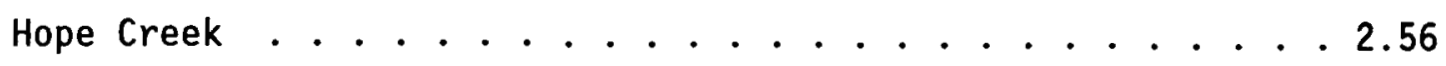
Indian Point $1,2, \& 3 \ldots \ldots . \ldots . \ldots . \ldots$ Kewaunee ......................... 2.60

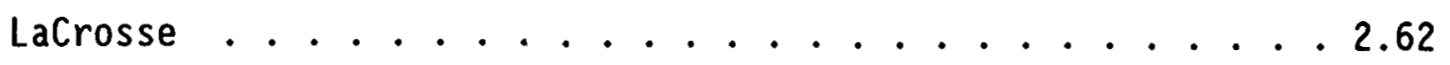
LaSalle $1 \& 2 \ldots \ldots . \ldots . \ldots . \ldots . \ldots$ Limerick . . . . . . . . . . . . . . 2.66

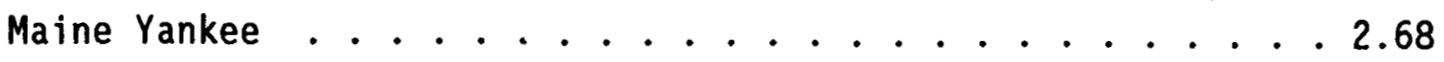
McGuire $1 \& 2 \ldots \ldots . \ldots . \ldots . \ldots . \ldots . \ldots$ Millstone $1,2 \& 3 \ldots \ldots . \ldots \ldots . \ldots \ldots$

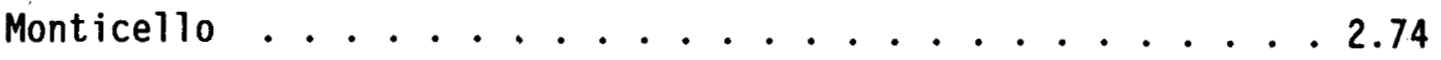
Nine Mile Point $1 \& 2 \ldots \ldots . \ldots . \ldots . \ldots$

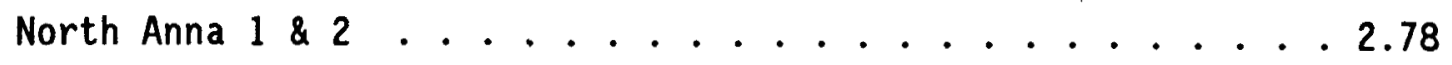
Oconee $1,2, \& 3 \ldots \ldots . \ldots \ldots$ 


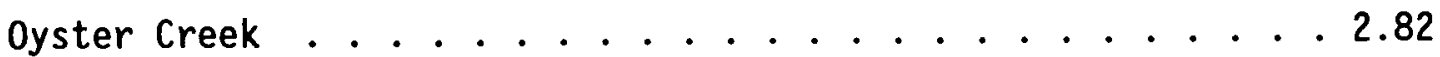

Palisades . . . . . . . . . . . . . . . 2.84

Palo Verde $1,2, \& 3 \ldots \ldots . \ldots . \ldots . \ldots 286$

Peach Bottom $2 \& 3 \ldots \ldots . \ldots . \ldots . \ldots . \ldots$

Perry ........................ 2.90

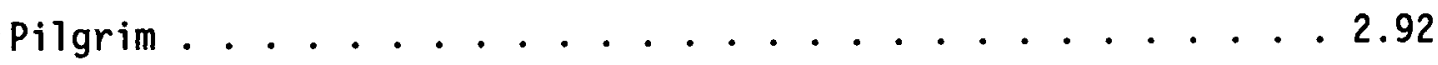

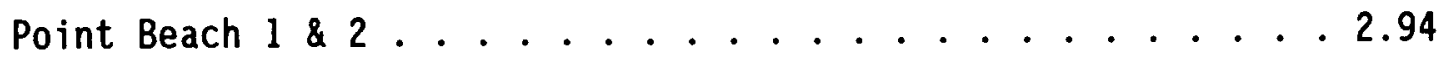

Prairie Island $1 \& 2 \ldots \ldots . \ldots . \ldots . \ldots . \ldots$

Quad Cities $1 \& 2 \ldots \ldots . \ldots . \ldots . \ldots . \ldots 98$

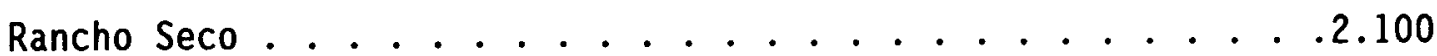

River Bend . . . . . . . . . . . . . 2. . . . . . . . . . .

H. B. Robinson 2....................... . . . . . .

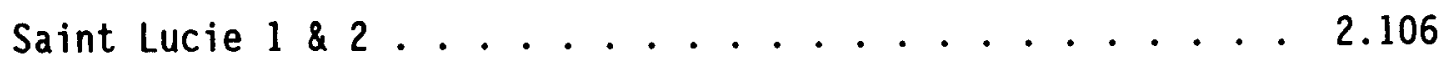

Salem $1 \& 2 \ldots \ldots \ldots . \ldots \ldots$

San Onofre 1, 2, \& $3 \ldots \ldots \ldots \ldots$

Sequoyah $1 \& 2 \ldots \ldots . \ldots \ldots . \ldots . \ldots . \ldots 2 . \ldots 112$

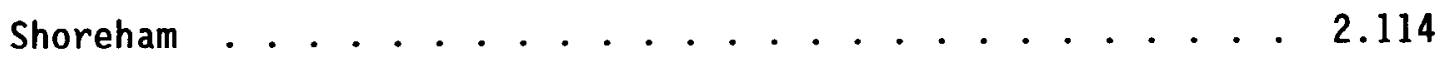

South Texas.................. 2.116

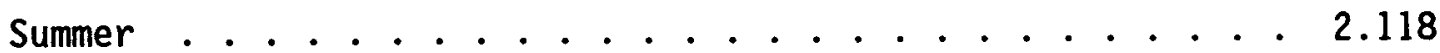

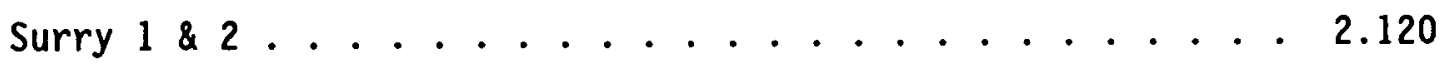

Susquehanna $1 \& 2 \ldots \ldots . \ldots . \ldots . . \ldots 2 . \ldots$

Three Mile Island $1 \& 2 \ldots \ldots . \ldots . \ldots . . \ldots 2 . \ldots$

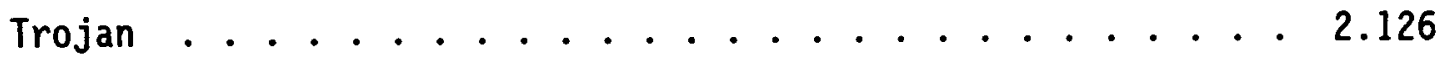

Turkey Point $3 \& 4 \ldots \ldots . \ldots . \ldots . . \ldots 2 . \ldots$ 
Vermont Yankee ................. 2.130

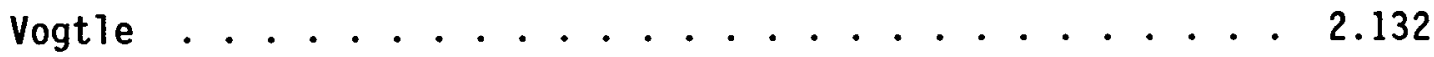

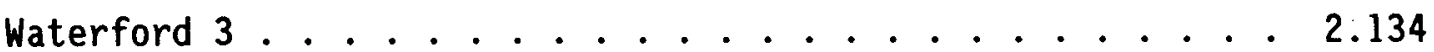

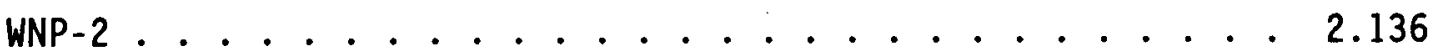

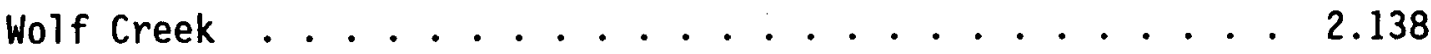

Yankee Rowe ................ . . 2. . . . . . . . . . .

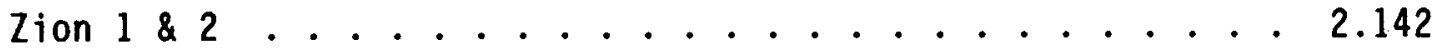

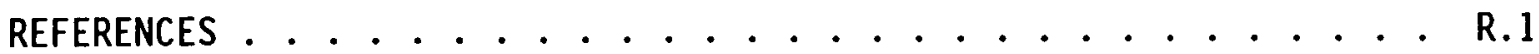

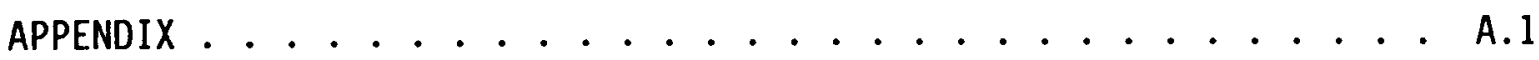




\section{FIGURES}

1. Fraction of Total Population Receiving Various Average Individual Total-Body Dose Commitments for All Sites . . . . . 1.8

2. Airborne Population Dose Commitment for the Reactor Sites, 19881.9 


\section{$\underline{\text { TABLES }}$}

1. Organs Considered in This Study . . . . . . . . . . . 1.1

2. Pathways Considered in This Study ............. 1.2

3. Environmental Statements for Power Plants Included in This Study 1.3

4. Reactor Characteristics and Population Total-Body Dose Commitments, 1988 ................. 1.4

5. Comparison of Annual Population Dose Commitments and Energy Output for the Past 14 Years . . . . . . . . . . . . . . 1.11

6. Total-Body Population Doses from Nuclear Power Plant Effluents During Normal Operations, 1975-1988 . . . . . . . . . . 1.13

7. Average Population Dose for Last Three Years, person-rem . . 1.14

8. Major Radionuclide Contributions to Population Doses from Liquid (L) and Air (A) Pathways ............. . 1.15 


\section{ACKNOWLEDGMENTS}

The author greatly appreciates the development of the population distributions around each site from census data by Donald P. Cleary of the U.S. Nuclear Regulatory Commission. The author also gratefully acknowledges the contributions and suggestions by Richard A. Hartfield, Thomas $H$. Essig, Charles A. Willis, and Kazimieras Campe, also of the U.S. Nuclear Regulatory Commission. 


\section{INTRODUCTION}

All commercial nuclear power reactors release small amounts of radioactive materials to the environment during normal operation. Because of these releases, concern was expressed about the magnitude of the collective dose received by the general population residing around these nuclear power plants. In response to this concern, the Pacific Northwest Laboratory (PNL) contracted with the Nuclear Regulatory Commission (NRC) to undertake a series of studies to estimate radiation dose commitments produced by radionuclide releases from commercial light-water power reactors starting in 1975 (see previous reports in this series, p. ii). This document is a continuation of these studies and considers the doses from releases during 1988. In this study, as in those previous, we estimated the collective (population) dose commitment(b) from both the liquid and gaseous releases to four age groups making up the population residing in the region of the site: infant ( 0 to $1 \mathrm{yr}$ ), child ( 1 to 11 $\mathrm{yr}$ ), teen-ager (11 to $17 \mathrm{yr}$ ), and adult (17 $\mathrm{yr}$ and $01 \mathrm{lder}$ ).

The particular organs of reference in this study are listed in Table 1. The major pathways by which radionuclides travel from the reactor to the individual receptors are shown in Table 2. Other possible liquid pathways such as direct exposure from waterborne activities (swimming, boating, shoreline recreation) and internal exposure through ingestion of food produced using contaminated irrigation water were not included; we have found from past experience that the doses from these pathways are generally much smaller than the doses from the pathways considered in this study.

\section{TABLE 1. Organs Considered in This Study}

\section{Organs Affected by \\ Airborne Releases}

Total body

Thyroid

Bone

GI tract

Liver

Lung
Organs Affected by Waterborne Releases

Total body

Thyroid

Bone

GI tract

Liver

(a) Operated by Battelle Memorial Institute for the Department of Energy.

(b) As used in this report, dose commitment describes the total-body or specified organ dose equivalent in rem ( 1 rem $=0.01$ sievert) received over 50 years from intake during the year in which radioactive materials were released into the environment from the power plants. 
TABLE 2. Pathways Considered in This Study

Pathways for

Airborne Releases

Air submersion

Ground irradiation

Inhalation

Ingestion of food crops

and animal products
Pathways for

Waterborne Releases

Ingestion of drinking water

Ingestion of fish and

invertebrates

The "source terms" used to estimate dose commitments produced from each site were the annual measured releases of radioactive materials as reported to the NRC by the plant operators and subsequently published in an NRC public document (Tichler, et al. 1991). Doses from carbon-14 generally are not included, because carbon-14 releases from most plants are not measured or reported.

The regional population for which we estimated dose commitments included those persons estimated to be living in a region between 2 and 80 $\mathrm{km}$ around the reactor sites during 1988. Population distributions were supplied by the NRC's Office of Nuclear Reactor Regulation. Atmospheric transport factors (annual average dilution and annual average deposition) were calculated for the region around each site using appropriate meteorological data supplied by the NRC or the utility. To calculate the doses, we used models approved by the NRC. We incorporated these models into two computer programs to expedite the dose calculations involved for each site.

Site-specific parameters other than releases, meteorology, and population were obtained from environmental statements [both final (FES) and draft (DES)] for the various reactors when available (see Table 3 ). Such parameter values include the total population drinking contaminated water, river flow, dilution flow from the reactors (for sites not on rivers), fish and invertebrate harvest for the region, and dilution factors for drinking water and aquatic foods. In those cases in which site-specific data are not readily available and the particular pathway is not expected to result in a large dose, conservative assumptions have been used to estimate doses. The use of more realistic data should result in lower dose estimates in most cases.

The reactors included in this study, their type, licensed thermal power rating and net electrical output for 1988 are listed in Table 4. Populations at risk and the dose commitments derived in the study are also shown in this table. 


\section{TABLE 3. Environmental Statements for Power Plants Included in This Study}

\begin{tabular}{|c|c|}
\hline $\begin{array}{l}\text { Site } \\
\text { Number }\end{array}$ & Reactor site \\
\hline 1 & Blo Rock Point \\
\hline 2 & Browns ferry \\
\hline 3 & Cooper \\
\hline 4 & Dresden \\
\hline 3 & Beaver valley \\
\hline 6 & Humbo Idt Bay \\
\hline 7 & Lacrosse \\
\hline 8 & millstone Point \\
\hline 9 & mont lcello \\
\hline 10 & Nine Mile Point \\
\hline 11 & Oyster creek \\
\hline 12 & Peach Bottom \\
\hline 13 & Pilgrim \\
\hline 14 & Quad clties \\
\hline 13 & Vermont rankee \\
\hline 16 & st. Lucle \\
\hline 17 & Brunswick \\
\hline 18 & Duane Arnold \\
\hline 19 & J. A. Fitzpatrlck \\
\hline 20 & E. 1. Hatch \\
\hline 21 & Arkansas one \\
\hline 22 & $\begin{array}{l}\text { Connect I cut rankee } \\
\text { (Haddam Neck) }\end{array}$ \\
\hline 23 & Fort Calhoun \\
\hline 24 & H. B. Robinson \\
\hline 25 & Indian polnt \\
\hline 26 & Salem \\
\hline 27 & Kewa unee \\
\hline 28 & malne Yankee \\
\hline 29 & Oconee \\
\hline 30 & Palísades \\
\hline 31 & Point Beach \\
\hline 32 & Prairle isiand \\
\hline 33 & R. E. CInna \\
\hline 34 & San onofre \\
\hline 35 & Surry \\
\hline 36 & Three Mlle island \\
\hline 37 & Turkey Point \\
\hline 38 & Yankee Rowe \\
\hline 39 & zion \\
\hline 40 & calvert cilfts \\
\hline 41 & Cook \\
\hline 42 & Trojan \\
\hline 43 & Rancho seco \\
\hline 44 & Crystal River \\
\hline 45 & Davis-Besse \\
\hline 46 & J. M. Farley \\
\hline 47 & North Anna \\
\hline 48 & sequoyah \\
\hline 49 & Mcculte \\
\hline 50 & Lasalle \\
\hline 31 & Summer \\
\hline 52 & Susquehanna \\
\hline 53 & Grand culf \\
\hline 54 & Callaway \\
\hline 55 & Limer lick \\
\hline 56 & Dlablo Canyon \\
\hline 57 & WNP-2 \\
\hline 58 & Palo verde \\
\hline 59 & Byron \\
\hline 60 & waterford \\
\hline 61 & wolf Creek \\
\hline 62 & Catawba \\
\hline 63 & FermI \\
\hline 64 & Shorehan \\
\hline 65 & Hope Creek \\
\hline 66 & Perry \\
\hline 67 & River bend. \\
\hline 68 & Brafionood \\
\hline 69 & clinton \\
\hline 70 & Marris \\
\hline 71 & vogtle \\
\hline 72 & South Texas Prolect \\
\hline
\end{tabular}

\begin{tabular}{|c|c|c|c|}
\hline \multicolumn{2}{|c|}{$\begin{array}{l}\text { Docket } \\
\text { Number }\end{array}$} & \multicolumn{2}{|r|}{ Date } \\
\hline & & JuI & 71 \\
\hline $50-298$ & & Feb & 73 \\
\hline $50-237$ & . $50-249$ & Nov & 73 \\
\hline $50-334$ & & juI & 73 \\
\hline $50-409$ & & Jun & 76 \\
\hline 50-245. & $50-336$ & Jun & 73 \\
\hline $50-263$ & & Nov & 72 \\
\hline $50-220$ & & Jan & 74 \\
\hline $50-219$ & & Dec & 74 \\
\hline 50-277. & $50-278$ & Apr & 73 \\
\hline $50-293$ & & may & 72 \\
\hline $30-254$ & $50-265$ & Sep & 72 \\
\hline $50-271$ & & Jul & 72 \\
\hline $50-335$ & & Jun & 73 \\
\hline $50-324$ & $50-325$ & Jun & 73 \\
\hline $50-331$ & & $\operatorname{mar}$ & $\begin{array}{l}73 \\
73\end{array}$ \\
\hline $50-333$ & & $\operatorname{mar}$ & $\begin{array}{l}73 \\
72\end{array}$ \\
\hline $\begin{array}{l}50-321 \\
50-313\end{array}$ & & oct & $\begin{array}{l}72 \\
73 \text {. Sep } 72\end{array}$ \\
\hline $\begin{array}{l}50-313 \\
50-213\end{array}$ & $50-368$ & $\begin{array}{l}\text { Feb } \\
o c t\end{array}$ & 73 \\
\hline $50-285$ & & Aug & 72 \\
\hline $50-261$ & & Apr & 74 \\
\hline $50-247$ & & sep & 72 \\
\hline 50-272, & . $50-311$ & ADr & 73 \\
\hline $50-305$ & & Dec & 72 \\
\hline 50-309 & & Jut & 72 \\
\hline 50-269, & $, 50-270,50-287$ & mar & 72 \\
\hline 50-255 & & Jun & 72 \\
\hline $50-266$ & . 50-301 & may & 72 \\
\hline 50-282. & $50-306$ & may & 73 \\
\hline $50-244$ & & DeC & 73 \\
\hline $50-206$ & & oct & 73 \\
\hline $50-281$ & & Iun & 72 \\
\hline $50-289$ & & $\begin{array}{l}\text { Dec } \\
\text { feb }\end{array}$ & $\begin{array}{l}72 \\
72\end{array}$ \\
\hline $50-250$ & $50-251$ & feb & 72 \\
\hline $50-295$ & 50-304 & Dec & 72 \\
\hline $50-317$ & & Apr & 73 \\
\hline $50-315$ & & Aug & 73 \\
\hline $50-344$ & & Jan & 73 \\
\hline $50-312$ & & mar & 73 \\
\hline $50-302$ & & May & 73 \\
\hline $50-346$ & & Mar & 73 \\
\hline $50-348$ & . $50-364$ & Jun & 72 \\
\hline 30.338 & $\begin{array}{l}50-339 \\
50-328\end{array}$ & ADr & 73 \\
\hline 50-327. & $\begin{array}{r}50-328 \\
50-370\end{array}$ & Feb & 74 \\
\hline $50-369$ & $\begin{array}{r}50-370 \\
50-374\end{array}$ & ADr & $\begin{array}{l}76 \\
78\end{array}$ \\
\hline $\begin{array}{l}50-373 \\
50-395\end{array}$ & $50-374$ & $\begin{array}{l}\text { Nov } \\
\text { Jan }\end{array}$ & $\begin{array}{l}78 \\
73\end{array}$ \\
\hline 50-387. & 50-388 & Jun & 73 \\
\hline $50-416$ & $50-417$ & Aug & 73 \\
\hline $50-483$ & $50-486$ & $\operatorname{mar}$ & 75 \\
\hline $50-352$ & $50-353$ & nov & 73 \\
\hline $50-275$ & $50-323$ & may & 73 \\
\hline $50-397$ & & Dec & 72 \\
\hline $30-528 t$ & thru $50-530$ & sep & 75 \\
\hline $50-454$ & $50-455$ & jul & 74 \\
\hline $50-382$ & & mar & 73 \\
\hline $50-482$ & & Jun & 82 \\
\hline 50-413. & . $50-414$ & Dec & 73 \\
\hline $50-341$ & & Ju! & 72 \\
\hline $50-322$ & & SeD & 72 \\
\hline $50-354$ & . $50-355$ & Feb & 74 \\
\hline $50-440$ & $50-441$ & Apr & 74 \\
\hline $50-458$ & . 50-459 & Sep & 74 \\
\hline $50-456$ & $50-457$ & mat & 74 \\
\hline $\begin{array}{l}50-461 \\
50-400\end{array}$ & $\begin{array}{l}50-462 \\
\text { thru } 50-403\end{array}$ & $\begin{array}{l}\text { Oct } \\
\text { mar }\end{array}$ & $\begin{array}{l}74 \\
74\end{array}$ \\
\hline $50-424$ & $50-425$ & Mar & 74 \\
\hline $50-498$ & 50-499 & mar & 72 \\
\hline
\end{tabular}

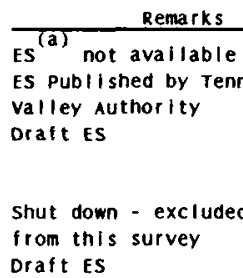

from this survey

Draft ES

Draft ES

FES of Indian Point 2 used

FES of Surry 2 used

Draft ES

ES not avallable

Draft ES

(a) Environmental statement.

(b) site added for 1988. 


\section{IABLE 4. Reactor Characteristics and Population Total-Body Dose Commitments, 1988}

\begin{tabular}{|c|c|c|c|c|c|c|c|c|c|}
\hline \multirow[b]{2}{*}{ site } & \multirow[b]{2}{*}{ Unit } & \multirow[b]{2}{*}{ Iype } & \multirow{2}{*}{$\begin{array}{c}\text { Licensed } \\
\text { Thermal } \\
\text { power } \\
\text { (Mw) } \\
\end{array}$} & \multirow{2}{*}{$\begin{array}{c}\text { Electric } \\
\text { Energy } \\
\text { ceneration } \\
1988 \text { (a) } \\
\text { (Tw.hr) } \\
\end{array}$} & \multicolumn{3}{|c|}{$\begin{array}{c}\text { Populat ion Dose Commi tment } \\
\text { (per son-rem) }\end{array}$} & \multirow{2}{*}{$\begin{array}{l}\text { Population } \\
\text { at Risk } \\
\end{array}$} & \multirow{2}{*}{$\begin{array}{c}\text { Average } \\
\text { Individual } \\
\text { Total-Body } \\
\text { Dose } \\
\text { Commi tment } \\
\text { (mr em) } \\
\end{array}$} \\
\hline & & & & & Llquid & $\Lambda \mid r^{(C)}$ & Total & & \\
\hline $\begin{array}{l}\text { Arkansas one } \\
\text { Arkansas one }\end{array}$ & $2^{1}$ TOTAL & $\begin{array}{l}\text { PWR } \\
\text { PWR }\end{array}$ & $\begin{array}{l}2568 \\
2815 \\
5383\end{array}$ & $\begin{array}{l}3.95 \\
4.95 \\
8.90\end{array}$ & 1.0 & 0.034 & 1.0 & $1.9 E 5$ & $5.4 E-3$ \\
\hline $\begin{array}{l}\text { Beaver valley } \\
\text { Beaver valley }\end{array}$ & $\stackrel{1}{2}_{\text {TOTAL }}$ & $\begin{array}{l}\text { PWR } \\
\text { PWR }\end{array}$ & $\begin{array}{l}2660 \\
2660 \\
7980\end{array}$ & $\begin{array}{r}4.98 \\
6.48 \\
11.46\end{array}$ & 0.046 & 0.085 & 0.13 & $3.6 \mathrm{E} 6$ & $3.6 \mathrm{E}-5$ \\
\hline B Ig Rock Point $(b)$ & 1 & BWR & 240 & 0.384 & 0.75 & 0.10 & 0.85 & $1.7 \mathrm{ES}$ & $5.1 E-3$ \\
\hline Braidwood & $2_{\text {TOTAL }}^{1}$ & $\begin{array}{l}\text { PWR } \\
\text { PWR }\end{array}$ & $\begin{array}{l}3411 \\
3411 \\
6822\end{array}$ & $\begin{array}{l}3.42 \\
1.35 \\
4.77\end{array}$ & 1.1 & 0.0061 & 1.1 & $4.3 \mathrm{E} 6$ & $2.6 E-4$ \\
\hline $\begin{array}{l}\text { Browns ferry } \\
\text { Browns ferry }\end{array}$ & ${ }^{1}$ TOTAL & $\begin{array}{l}\text { BWR } \\
\text { BWR } \\
B W R\end{array}$ & $\begin{array}{l}3293 \\
3293 \\
3293 \\
9879\end{array}$ & $\begin{array}{l}0 \\
0 . \\
0 . \\
0\end{array}$ & 1.2 & 0.0011 & 1.2 & $7.5 E 5$ & $1.6 \mathrm{E}-3$ \\
\hline $\begin{array}{l}\text { Brunswick } \\
\text { Brunswick }\end{array}$ & 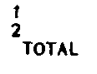 & $\begin{array}{l}\text { BWR } \\
\text { BWR }\end{array}$ & $\begin{array}{l}2436 \\
2436 \\
4872\end{array}$ & $\begin{array}{l}4.45 \\
3.92 \\
8.37\end{array}$ & 0.0047 & 0.35 & 0.040 & $2.4 E 5$ & $1.7 E-4$ \\
\hline $\begin{array}{l}\text { Byron } \\
\text { Byron }\end{array}$ & ${ }^{1}$ TOTAL & $\begin{array}{l}\text { PWR } \\
\text { PWR }\end{array}$ & $\begin{array}{l}3411 \\
3411 \\
6822\end{array}$ & $\begin{array}{r}6.29 \\
6.36 \\
12.65\end{array}$ & 0.092 & 0.029 & 0.12 & $9.4 E 5$ & $1.3 E-4$ \\
\hline Callaway & 1 & PWR & 3565 & 8.94 & 0.0001 & 0.0065 & 0.0066 & $3.7 E 5$ & $1.8 E-5$ \\
\hline $\begin{array}{l}\text { calvert clifts } \\
\text { calvert cliffs }\end{array}$ & ${ }^{1}$ TOTAL & $\begin{array}{l}\text { PWR } \\
\text { PWR }\end{array}$ & $\begin{array}{l}2700 \\
2700 \\
5400\end{array}$ & $\begin{array}{r}5.16 \\
6.60 \\
11.76\end{array}$ & 1.5 & 0.24 & 1.7 & $2.8 \mathrm{E} 6$ & $6.1 E-4$ \\
\hline $\begin{array}{l}\text { Catawba } \\
\text { Catawba }\end{array}$ & ${ }^{1}$ TOTAL & $\begin{array}{l}\text { PWR } \\
\text { PWR }\end{array}$ & $\begin{array}{l}3411 \\
3411\end{array}$ & $\begin{array}{r}7.63 \\
6.17 \\
13.80\end{array}$ & 1.4 & 0.21 & 1.6 & $1.6 \mathrm{E} 6$ & $1.0 E-3$ \\
\hline clinton & 1 & 8WR & 2894 & 5.86 & 4.0 & 0.0087 & 4.0 & $2.7 E+6$ & $1.5 E-3$ \\
\hline $\begin{array}{l}\text { cook } \\
\text { cook }\end{array}$ & ${ }^{1}$ TOTAL & $\begin{array}{l}\text { PWR } \\
\text { PWR }\end{array}$ & $\begin{array}{l}3250 \\
3391 \\
6641\end{array}$ & $\begin{array}{l}7.47 \\
2.32 \\
9.79\end{array}$ & 0.21 & 0.0059 & 0.22 & $1.2 \mathrm{E} 6$ & $1.9 E-4$ \\
\hline Cooper & 1 & BWR & 2381 & 4.20 & 0.0068 & 0.0049 & 0.012 & $1.7 \mathrm{E} 5$ & $6.8 E-5$ \\
\hline Crystal River & 3 & PWR & 2452 & 5.77 & 0.19 & 0.011 & 0.20 & $4.6 \mathrm{E} 5$ & $4.4 E-4$ \\
\hline Davis-Besse & 1 & PWR & 2772 & 1.16 & 0.0018 & 0.016 & 0.018 & $1.8 E 6$ & $9.9 E-6$ \\
\hline $\begin{array}{l}\text { Diablo Canyon } \\
\text { Diablo Canyon }\end{array}$ & ${ }^{1}$ тоTAL & $\begin{array}{l}\text { PWR } \\
\text { PWR }\end{array}$ & $\begin{array}{l}3338 \\
3411 \\
6749\end{array}$ & $\begin{array}{r}5.26 \\
6.23 \\
11.49\end{array}$ & 0.0063 & 0.015 & 0.021 & $3.1 \mathrm{E} 5$ & 6. $9 E-5$ \\
\hline $\begin{array}{l}\text { Dresden } \\
\text { Dresden }\end{array}$ & $\stackrel{1}{2}^{\frac{1}{3}}$ & $\begin{array}{l}\text { BWR } \\
\text { BWR } \\
\text { BWR }\end{array}$ & $\begin{array}{l}700 \\
2527 \\
2527 \\
5754\end{array}$ & $\begin{array}{l}0 . \\
4.32 \\
4.16 \\
8.48\end{array}$ & o & 0.11 & 0.11 & $6.5 \mathrm{E} 6$ & $1.7 E-5$ \\
\hline Duane Arnold & 1 & BWR & 1658 & 3.14 & 0 & 0.064 & 0.064 & $5.8 \mathrm{E} 5$ & 1. $1 E-4$ \\
\hline $\begin{array}{l}\text { J. M. Farley } \\
\text { J. M. Fafley }\end{array}$ & ${ }^{1}$ TOTAL & $\begin{array}{l}\text { PWR } \\
\text { PWR }\end{array}$ & $\begin{array}{l}\mathbf{2 6 5 2} \\
\mathbf{2 6 5 2} \\
\mathbf{5 3 0 4}\end{array}$ & $\begin{array}{r}5.91 \\
7.17 \\
13.08\end{array}$ & 0.020 & 0.045 & 0.065 & $3.7 E 5$ & $1,7 \varepsilon-4$ \\
\hline Fermi & 2 & BWR & 3292 & 4.06 & 0.010 & 0.0024 & 0.012 & 4. $9 E 6$ & $2.5 E-6$ \\
\hline J. A. Fitzpatrick & 1 & BWR & 2436 & $4: 36$ & 0.017 & 0.14 & 0.16 & $8.6 \mathrm{E} 5$ & $1.8 E-4$ \\
\hline Fort Calhoun & 1 & PWR & 1500 & 2.63 & 0.33 & $0.0 r 80$ & 0.34 & $7.6 \mathrm{ES}$ & $4.4 E-4$ \\
\hline R. E. Ginna & 1 & PWR & 1520 & 3.53 & 0. 16 & 0.073 & 0.23 & $1.2 E 6$ & $1.9 E-4$ \\
\hline Grand Culf & 1 & BWR & 3833 & 9.59 & 0.0004 & 0.0007 & 0.0011 & $3.4 \mathrm{E} 5$ & $3.2 E-6$ \\
\hline Haddam Neck & 1 & PWR & 1825 & 3.31 & 0.077 & 0.46 & 0.54 & $3.5 E 6$ & $1.5 E-4$ \\
\hline Harris & 1 & PWR & 2775 & 5.33 & 1.7 & 0.10 & 1.8 & $1.4 E 6$ & $1.3 E-3$ \\
\hline $\begin{array}{l}\text { E. H. Hatch } \\
\text { E. I. Hatch }\end{array}$ & ${ }^{1}$ TOTAL & $\begin{array}{l}\text { BWR } \\
\text { BWR }\end{array}$ & $\begin{array}{l}2436 \\
2436 \\
4877\end{array}$ & $\begin{array}{l}4.11 \\
4.25 \\
8.36\end{array}$ & 6.4 & 0.035 & 6.4 & $3.5 E 5$ & $1.6 E-2$ \\
\hline Hope Creek & 1 & BWR & 3293 & 6.99 & 0.96 & 0.13 & 1.1 & 4. $9 \mathrm{E} 6$ & $2.2 E-4$ \\
\hline $\begin{array}{l}\text { Indian Point } \\
\text { Indian point }\end{array}$ & $\begin{array}{l}1 \\
2 \\
3 \\
\text { TOTAL }\end{array}$ & $\begin{array}{l}\text { PWR } \\
\text { PWR } \\
\text { PWR }\end{array}$ & $\begin{array}{r}615 \\
2758 \\
2760 \\
6133\end{array}$ & $\begin{array}{r}0 . \\
6.06 \\
6.71 \\
12.77\end{array}$ & 0.092 & 0.34 & 0.43 & $1.6 \mathrm{E7}$ & $2.8 E-5$ \\
\hline Kewaunee & 1 & PWR & 1650 & 3.91 & 1.3 & 0.0022 & 1.3 & $6.3 E 5$ & 2. 1E-3 \\
\hline Lacrosse $e^{(b)}$ & 1 & BWR & 165 & 0. & 0.41 & 0.0003 & 0.41 & $3.5 E 5$ & $1.2 E-3$ \\
\hline $\begin{array}{l}\text { Lasalle } \\
\text { Lasalle }\end{array}$ & 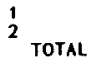 & $\begin{array}{l}\text { BWR } \\
\text { BWR }\end{array}$ & $\begin{array}{l}3323 \\
3323 \\
6646\end{array}$ & $\begin{array}{r}5.44 \\
5.66 \\
11.10\end{array}$ & 0 & 0.27 & 0.27 & 1. 1E6 & $2.5 E-4$ \\
\hline Limer ick & & BWR & 3293 & 6.67 & 0 & 0.25 & 0.25 & $6.9 E 6$ & $3.6 \mathrm{E}-5$ \\
\hline maine rankee & 1 & PWR & 2630 & 5.02 & 0.0029 & 0.0047 & 0.0076 & $6.3 \mathrm{E} 5$ & 1. $2 \mathrm{E}-5$ \\
\hline $\begin{array}{l}\text { Mccuire } \\
\text { mccuire }\end{array}$ & $2^{1}$ TOTAL & $\begin{array}{l}\text { PWR } \\
\text { PWR }\end{array}$ & $\begin{array}{l}3411 \\
3411 \\
6822\end{array}$ & $\begin{array}{r}7.39 \\
8.05 \\
15.44\end{array}$ & 16. & 0.17 & 16. & $1.8 E 6$ & 9. IE-3 \\
\hline $\begin{array}{l}\text { Millstone } \\
\text { millstone }\end{array}$ & $\begin{array}{l}1 \\
2 \\
3 \\
\text { TOTAL }\end{array}$ & $\begin{array}{l}\text { BWR } \\
\text { PWR } \\
\text { PWR }\end{array}$ & $\begin{array}{l}2011 \\
2700 \\
3411 \\
4571\end{array}$ & $\begin{array}{r}5.54 \\
5.73 \\
7.67 \\
18.94\end{array}$ & 0.0027 & 0.22 & 0.22 & $2.6 \mathrm{E} 6$ & $8.5 \mathrm{E}-5$ \\
\hline monticello & 1 & BWR & 1670 & 4.57 & $\mathbf{0}$ & 0.18 & 0.18 & $2.3 E 6$ & $8.0 E-5$ \\
\hline $\begin{array}{l}\text { Nine mile Point } \\
\text { Nine mile Point }\end{array}$ & $i_{\text {TOTAL }}^{1}$ & $\begin{array}{l}\text { BWR } \\
\text { BWR }\end{array}$ & $\begin{array}{l}1850 \\
3323 \\
5173\end{array}$ & $\begin{array}{l}0 . \\
2.51 \\
2.51\end{array}$ & 0.21 & 0.0044 & 0.21 & $8.6 E 5$ & $2.5 E-4$ \\
\hline
\end{tabular}


IABLE 4. (Contd)

\begin{tabular}{|c|c|c|c|c|c|c|c|c|c|}
\hline \multirow[b]{2}{*}{ site } & \multirow[b]{2}{*}{$\underline{\text { Unit }}$} & \multirow[b]{2}{*}{ Type } & \multirow{2}{*}{$\begin{array}{c}\text { Licensed } \\
\text { Thermal } \\
\text { Power } \\
(\text { MW }) \\
\end{array}$} & \multirow{2}{*}{$\begin{array}{r}\text { Electric } \\
\text { Energy } \\
\text { Generation } \\
1988 \text { (a) } \\
(T w . h r)(a) \\
\end{array}$} & \multicolumn{3}{|c|}{$\begin{array}{c}\text { Populat ion Dose commitment } \\
\text { (person-rem) }\end{array}$} & \multirow{2}{*}{$\begin{array}{l}\text { Population } \\
\text { at Risk } \\
\end{array}$} & \multirow{2}{*}{$\begin{array}{c}\text { Average } \\
\text { Individual } \\
\text { Total-Body } \\
\text { Dose } \\
\text { Comititment } \\
\text { (mrem) } \\
\end{array}$} \\
\hline & & & & & Liquid & $\operatorname{Alr}(C)$ & Total & & \\
\hline $\begin{array}{l}\text { Nor th Anna } \\
\text { North Anna }\end{array}$ & $\begin{array}{l}1 \\
2 \\
\\
\text { TOTAL }\end{array}$ & $\begin{array}{l}\text { PWR } \\
\text { PWR }\end{array}$ & $\begin{array}{l}2893 \\
2893 \\
5786\end{array}$ & $\begin{array}{r}6.90 \\
7.88 \\
14.78\end{array}$ & 0.01 & 0.026 & 0.036 & $1.2 \mathrm{E} 6$ & $3.1 E-5$ \\
\hline $\begin{array}{l}\text { Oconee } \\
\text { oconee }\end{array}$ & $\begin{array}{l}1 \\
2 \\
3 \\
\end{array}$ & $\begin{array}{l}\text { PWR } \\
\text { PPWR } \\
\text { PWR }\end{array}$ & $\begin{array}{l}2568 \\
2568 \\
2568 \\
7704\end{array}$ & $\begin{array}{r}7.19 \\
5.54 \\
5.97 \\
18.70\end{array}$ & 3.3 & 0.54 & 3.8 & $9.9 E 5$ & $3.9 F-3$ \\
\hline oyster creek & 1 & BWR & 1930 & 3.54 & 0.20 & 2.0 & 2.2 & $3.6 \mathrm{E} 6$ & $6.0 \mathrm{E}-4$ \\
\hline Pal/sades & 1 & PWR & 2530 & 3.44 & 0.19 & 0.025 & 0.22 & $1.1 E 6$ & $2.0 E-4$ \\
\hline Palo verde & $\begin{array}{ll}1 & \\
2 & \\
3 & \text { TOTAL }\end{array}$ & $\begin{array}{l}\text { PWR } \\
\text { PWR } \\
\text { PWR }\end{array}$ & $\begin{array}{r}3800 \\
3800 \\
3800 \\
11400\end{array}$ & $\begin{array}{r}6.67 \\
6.75 \\
0.04 \\
13.46\end{array}$ & 0 & 0.20 & 0.20 & 1. $2 \mathrm{E} 6$ & $1.7 E-4$ \\
\hline $\begin{array}{l}\text { Peach Bottom } \\
\text { Peach Bottom }\end{array}$ & ${ }^{2}$ TOTAL & $\begin{array}{l}\text { BWR } \\
\text { BWR }\end{array}$ & $\begin{array}{l}3293 \\
3293 \\
6586\end{array}$ & :. & 0.32 & 0.014 & 0.33 & $4.3 \mathrm{E} 6$ & $7.7 E-5$ \\
\hline Periy & 1 & BWR & 3579 & 7.23 & 0.11 & 0.068 & 0.18 & $2.4 E 6$ & $7.5 E-5$ \\
\hline Pilgria & 1 & BWR & 1998 & 0 & 0.0014 & 0.0006 & 0.0020 & $4.4 E 6$ & $4.6 E-7$ \\
\hline $\begin{array}{l}\text { Point Beach } \\
\text { Point Beach }\end{array}$ & $2^{1}$ TOTAL & $\begin{array}{l}\text { PWR } \\
\text { PWR }\end{array}$ & $\begin{array}{l}1518 \\
1518 \\
3036\end{array}$ & $\begin{array}{l}3.83 \\
3.72 \\
7.55\end{array}$ & 0.017 & 0.030 & 0.047 & $6.4 E 5$ & $7.4 E-5$ \\
\hline $\begin{array}{l}\text { Prairle istand } \\
\text { Prairle island }\end{array}$ & ${ }^{1}$ TотAL & $\begin{array}{l}\text { PWR } \\
\text { PWR }\end{array}$ & $\begin{array}{l}1650 \\
1650 \\
3300\end{array}$ & $\begin{array}{l}3.82 \\
3.89 \\
7.71\end{array}$ & 0.0031 & 0.077 & 0.080 & $2.3 \mathrm{E} 6$ & $3.5 E-5$ \\
\hline $\begin{array}{l}\text { Quad cities } \\
\text { Quad cities }\end{array}$ & $\frac{1}{2}$ TOTAL & $\underset{\text { BWR }}{\text { BWR }}$ & $\begin{array}{l}2511 \\
2511 \\
5022\end{array}$ & $\begin{array}{l}5.66 \\
4.18 \\
9.84\end{array}$ & 0.040 & 0.016 & 0.056 & $7.3 \mathrm{E} 5$ & $7 \cdot 3 E-5$ \\
\hline Rancho Seco & 1 & PWR & 2772 & 2.81 & 0.021 & 0.059 & 0.080 & 2. $1 E 6$ & $3.8 E-5$ \\
\hline River Bend & 1 & BWR & 2894 & 7.25 & 0.0007 & 0.0010 & 0.0017 & $7.6 E 5$ & $2.2 E-6$ \\
\hline H. B. Robinson & 2 & PWR & 2300 & 3.18 & 0.041 & 0.020 & 0.061 & $7.3 E 5$ & $8 \cdot 3 E-5$ \\
\hline $\begin{array}{l}\text { St. Lucie } \\
\text { st. Lucie }\end{array}$ & $\stackrel{1}{2}$ TOTAL & $\begin{array}{l}\text { PWR } \\
\text { PWR }\end{array}$ & $\begin{array}{l}2560 \\
2700 \\
5260\end{array}$ & $\begin{array}{r}6.25 \\
7.41 \\
13.66\end{array}$ & 0.0002 & 0.36 & 0.36 & $7.2 \mathrm{E} 5$ & $5.0 E-4$ \\
\hline $\begin{array}{l}\text { sa lem } \\
\text { sa lem }\end{array}$ & $2^{1}$ TOTAL & $\begin{array}{l}\text { PWR } \\
\text { PWR }\end{array}$ & $\begin{array}{l}3338 \\
3338 \\
6676\end{array}$ & $\begin{array}{r}7.41 \\
5.97 \\
13.38\end{array}$ & 0.28 & 0.66 & 0.94 & 4. $9 \mathrm{E} 6$ & $1.9 E-4$ \\
\hline $\begin{array}{l}\text { San onofre } \\
\text { san onofre }\end{array}$ & 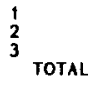 & $\begin{array}{l}\text { PWR } \\
\text { PWFR } \\
\text { PWR }\end{array}$ & $\begin{array}{l}1347 \\
3410 \\
3390 \\
8147\end{array}$ & $\begin{array}{r}1.37 \\
9.00 \\
6.13 \\
16.50\end{array}$ & 0.023 & 0.48 & 0.50 & $5.5 E 6$ & $9.1 E-5$ \\
\hline $\begin{array}{l}\text { Sequoyah } \\
\text { sequoyah }\end{array}$ & $\mathfrak{1}^{1}$ TOTAL & $\begin{array}{l}\text { PWR } \\
\text { PWR }\end{array}$ & $\begin{array}{l}2815 \\
2815 \\
5630\end{array}$ & $\begin{array}{l}0.07 \\
3.88 \\
3.95\end{array}$ & 0.26 & 0.019 & 0.28 & $8.8 \mathrm{E5}$ & $3.2 \mathrm{E}-4$ \\
\hline Shoreham & 1 & BWR & 121.8 & 0 & 0.0015 & 0 & 0.0015 & $5.2 \mathrm{E} 6$ & $2.9 E-7$ \\
\hline South Texas & 1 & PWR & $\mathbf{3 8 0 0}$ & 2.79 & 0.14 & 0.028 & 0.17 & $2.8 \mathrm{E} 5$ & 6. $1 E-4$ \\
\hline Summer & 1 & PWR & 2775 & 5.05 & 13 & 0.0070 & 13. & $9.0 \mathrm{E} 5$ & $1.4 E-2$ \\
\hline $\begin{array}{l}\text { Surry } \\
\text { surry }\end{array}$ & $\mathfrak{1}^{1}$ TOTAL & $\begin{array}{l}\text { PWR } \\
\text { PWR }\end{array}$ & $\begin{array}{l}2441 \\
2441 \\
4882\end{array}$ & $\begin{array}{l}2.69 \\
3.57 \\
6.26\end{array}$ & 0.47 & 0.040 & 0.51 & $1.9 E 6$ & $2.7 E-4$ \\
\hline $\begin{array}{l}\text { Susquehanna } \\
\text { susquehanna }\end{array}$ & $2^{1}$ TOIAL & $\begin{array}{l}\text { BWR } \\
\text { BWR }\end{array}$ & $\begin{array}{l}3293 \\
3293 \\
6586\end{array}$ & $\begin{array}{r}8.41 \\
5.90 \\
14.31\end{array}$ & 0.018 & 0.016 & 0.034 & $1.5 \mathrm{EG}$ & $2.3 E-5$ \\
\hline $\begin{array}{l}\text { Three mile Istand } \\
\text { Three Mile island }\end{array}$ & $\frac{1}{2}$ & $\underset{\text { PWR }}{\text { PWR }}$ & $\begin{array}{l}2535 \\
2772 \\
5307\end{array}$ & $\begin{array}{l}5.47 \\
0.47 \\
5.47\end{array}$ & 0.12 & 0.22 & 0.34 & $2.1 E 6$ & $1.6 E-4$ \\
\hline Trojan & 1 & PWR & 3411 & 6.34 & 0.0013 & 0.045 & 0.046 & $1.5 \mathrm{E} 6$ & 3. $1 E-5$ \\
\hline $\begin{array}{l}\text { Turkey Point } \\
\text { Turkey Point }\end{array}$ & ${ }^{3}$ TOTAL & $\begin{array}{l}\text { PWR } \\
\text { PWR }\end{array}$ & $\begin{array}{l}2200 \\
2200 \\
4400\end{array}$ & $\begin{array}{l}3.45 \\
3.26 \\
6.71\end{array}$ & 0.045 & 0.14 & 0.18 & 3.056 & $6.3 E-5$ \\
\hline vermont rankee & 1 & BWR & 1593 & 4.11 & 0 & 0.059 & 0.059 & $1.4 E 6$ & $4 \cdot 1 E-5$ \\
\hline Vogtle & 1 & PWR & 3411 & 6.79 & 0.0029 & 0.018 & 0.021 & $6.2 E 5$ & $3.4 E-5$ \\
\hline Waterford & 3 & PWR & 3390 & 6.54 & 0.063 & 0.48 & 0.54 & $1.8 \mathrm{E} 6$ & $3.0 E-4$ \\
\hline WNP- 2 & & BWR & 3323 & 6.00 & 0.0002 & 0.029 & 0.029 & $2.8 \mathrm{E} 5$ & 1. $1 E-4$ \\
\hline wolt creek & 1 & PWR & 3411 & 6.66 & 0.18 & 0.011 & 0.19 & $1.9 \mathrm{ES}$ & $1.0 E-3$ \\
\hline Vankee Rowe & 1 & PWR & 600 & 1.12 & 0.45 & 0.038 & 0.49 & $1.6 \mathrm{E} 6$ & $3.0 E-4$ \\
\hline $\begin{array}{l}\text { zion } \\
\text { zion }\end{array}$ & $\mathfrak{1}^{1}$ TOTAL & $\begin{array}{l}\text { PWR } \\
\text { PWR }\end{array}$ & $\begin{array}{l}3250 \\
3250 \\
6500\end{array}$ & $\begin{array}{r}6.34 \\
6.65 \\
12.99 \\
\end{array}$ & 6.8 & 0.44 & 7.2 & $7.3 \mathrm{E} 6$ & $1.0 E-3$ \\
\hline TOTAL FOR ALL SITES & & & & 511.21 & 65. & 9.6 & 75 & $1.5 \mathrm{~EB}$ & $\cdots$ \\
\hline Ar ithmetic mean & & & & 7.2 & 0.92 & 0.14 & 1.1 & 2. $1 E 6$ & $1.1 E-3$ \\
\hline Geometric mean & & & & 0.80 & 0.086 & 0.038 & 0.18 & 1. $2 \mathrm{E} 6$ & $1.5 E-4$ \\
\hline
\end{tabular}

(a) $1 \mathrm{Tw}-\mathrm{hr}=3.6 \mathrm{E} 15$ joules.

(b) Does not have charcoal delay beds in the gaseous effluent line from a ir ejector. 


\section{Site-Dependent Parameters}

In the Site Summaries section, the location (including latitude and longitude) for each reactor site and the estimated 1988 population within 2 to $80 \mathrm{~km}$ around each site is given. In addition, the locations of major metropolitan centers within $80 \mathrm{~km}$ are listed along with their 1988 extrapolated populations. The populations of the Standard Metropolitan Statistical Areas (SMSA) are given where applicable. Next, the site-specific data pertinent to the airborne pathways are specified. The average production rates of vegetable crops and animal products are given for the area within an $80-\mathrm{km}$ radius based upon the statewide average. This production has been reduced for sites on lakes and seacoasts to account for the presence of the body of water. An animal grazing factor is estimated for each site location. This factor accounts for the fraction of the year during which grazing animals such as milk cows and beef cattle graze on fresh pasture in the region around the site. After average production rates are given, the period of record and the percent data recovery of the meteorological data used in calculating diffusion factors are indicated.

Various site-dependent factors associated with the waterborne pathways are presented next. For lake and ocean sites, we used the average dilution of plant effluents for the year 1988 specified by Tichler, et al. (1991). For river sites, the average annual river flow is tabulated. This flow was used in place of a dilution flow from the plant to account for dilutions of liquid releases at the locations of probable intake of drinking water and aquatic food catch. Any exceptions to this scheme have been footnoted. Next is shown the estimated 1988 population utilizing drinking water drawn from supplies containing diluted effluents from the site. These are shown with an estimated dilution factor where applicable. Fish and invertebrate catch data taken from the respective plant environmental statement, when available (see Table 3 ), are listed next, along with estimated dilution factors for the lake and ocean sites. When site-specific fish and invertebrate catch data were not available, the generic consumption rates were used for the particular site. Sites on salt water were assumed to contribute no dose from drinking water. Any invertebrate catch from fresh water was not considered. 


\section{RESULTS}

This report consists of a summary of values used for site-specific parameters at each site, as explained above, and the results of population dose commitment calculations. The population dose commitments are presented in two tables facing the page summarizing site-specific parameters for that site. These tables include both waterborne and airborne pathway dose commitments for the several organs of reference for each age group investigated. They also include the dose to the whole population, which includes all age groups. The airborne population dose commitments for each of 160 segments (a) partitioning the region around the site were divided by the population residing within that segment to derive an average individual dose for that segment. These doses are summarized as a histogram showing percent of the population receiving a given dose level for each site. The fractional population dose from the waterborne pathway was not determined in this manner, because the NRC does not at present take into account the location of individuals exposed via this pathway, except those exposed through ingestion of drinking water.

Population dose commitments estimated for both the waterborne pathways and airborne pathways varied widely over the sites studied. The total dose commitments (from both pathways) varied from a high of 16 to a low of 0.0011 person-rem for plants in operation during the whole year. The arithmetic mean for the total dose from 1 iquid pathways (0.92 person-rem) and airborne pathways (0.14 person-rem) was 1.1 person-rem (see Table 4).

As in past years, most of the plants accounted for less than a personrem to their surrounding population from plant releases of radio- nuclides into liquid effluent streams. Only two sites had waterborne releases that resulted in population doses exceeding 10 person-rem. The sites were McGuire with 16 person-rem and Summer with 13. These waterborne doses were due almost totally to tritium via the drinking water pathway in the case of McGuire and cesium from fish consumption in the case of Summer.

The doses from radionuclide releases from plant vents and/or stacks into the atmosphere also accounted for less than a person-rem for most sites. Only one site had airborne releases resulting in 1 or more personrem. The site was oyster Creek with 2 person-rem. This airborne dose was the result of krypton- 88 and Xenon-135.

The total population dose commitments from all sites for 1988 were estimated to be 65 person-rem via waterborne pathways and 10 person-rem via airborne pathways for a total of 75 person-rem (see Table 4).

(a) See Appendix for definition of segments. 
Figure 1 is a histogram for all sites taken together. We can see from this plot that about $97 \%$ of the total population at risk from airborne releases (140 million) would each receive a dose commitment of less than 3 $\times 10^{-4}$ mrem. We can see further that about $9 \%$ receive a dose which is less than $1 \times 10^{-6} \mathrm{mrem}$. However, no attempt was made in this study to estimate the maximum dose commitment received by any one individual from the radionuclides released at any of the sites. However, licensee calculation of doses to the maximally exposed individual at some sites indicated values of up to approximately 100 times average individual doses (on the order of a few millirem per year).

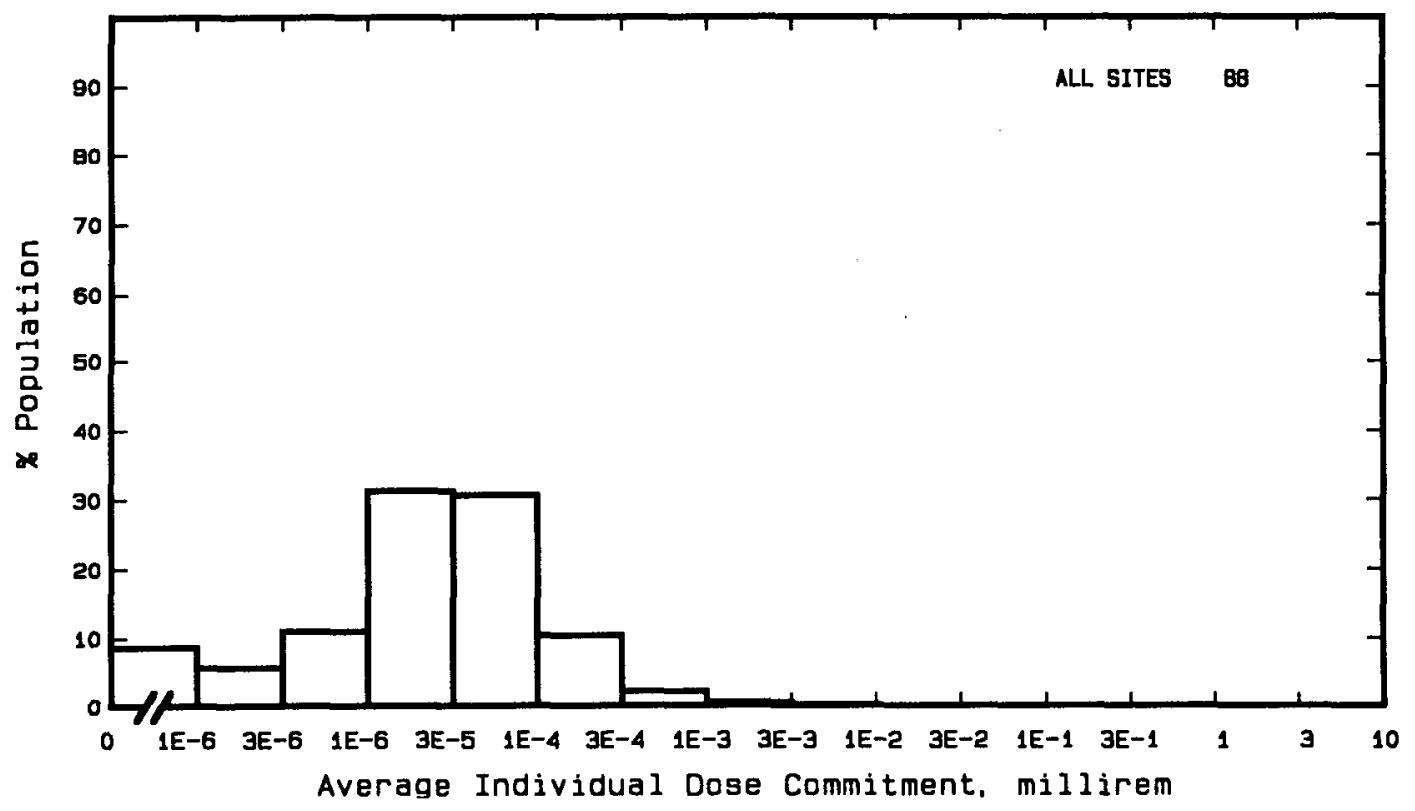

FIGURE 1. Fraction of Total Population Receiving Various Average
Individual Total-Body Dose Commitments for All Sites

Figure 2 shows graphically by "Box-and-Whisker Plots" the wide range of the airborne population dose commitments for the reactor sites. The median, upper and lower quartiles and upper and lower octiles for the distribution of doses calculated for each of 160 segments are indicated for each site. The upper and lower octiles are shown as the horizontal bars on the "whiskers;" the upper and lower quartiles are indicated as the top and bottom of the "box." The median is indicated as the horizontal line inside the box. No airborne releases were reported for the Shoreham site for 1988; therefore, none are plotted. 


\section{$6^{\circ} \mathrm{I}$}

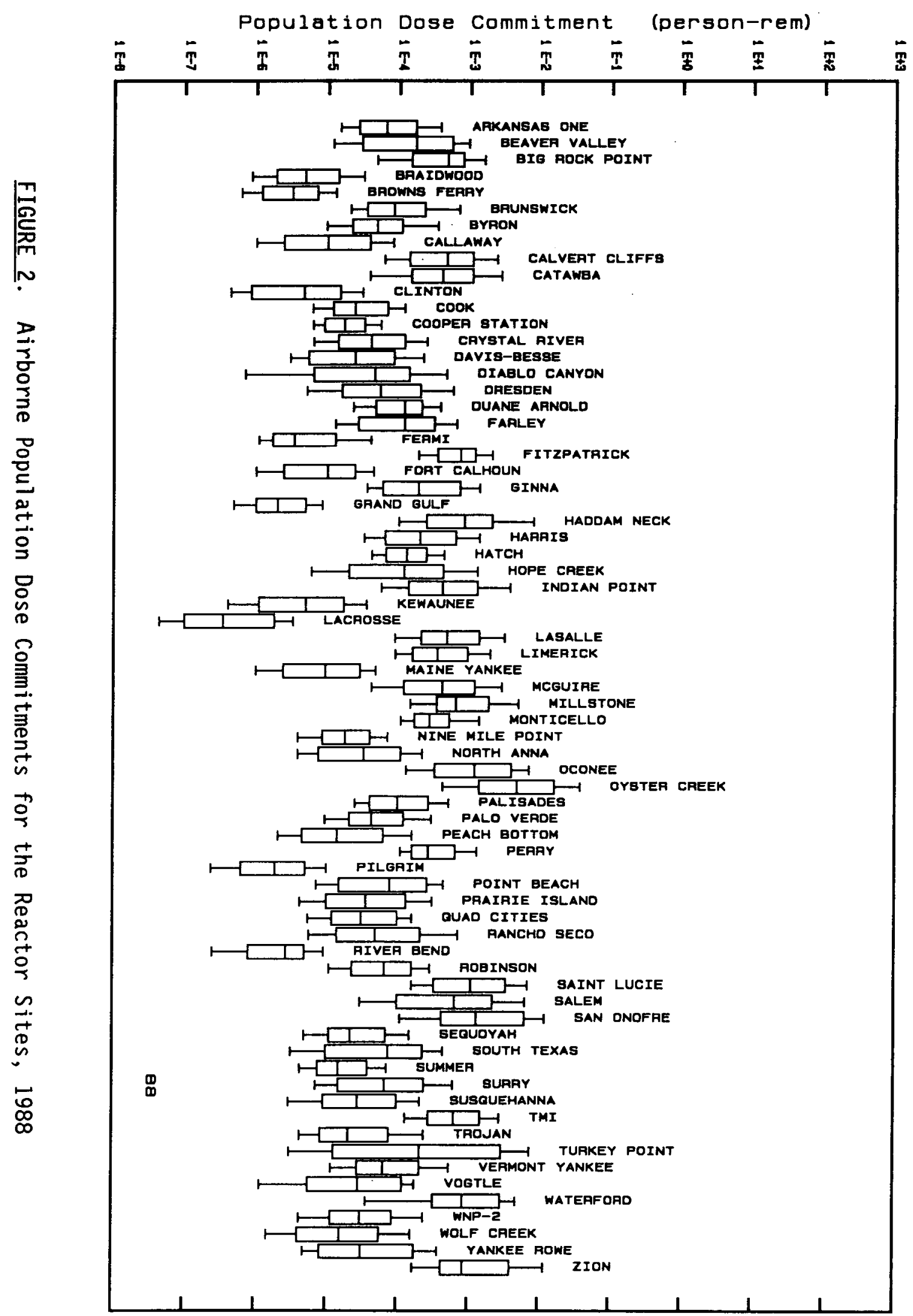


We should point out here, however, that the doses estimated in this study are extremely low compared to an average annual background dose of approximately $100 \mathrm{mrem}$ (neglecting radon daughters). We have compared dose commitments calculated in this study with annual background. However, this comparison is not quite exact, since these dose commitments are those total-body doses received from the year's effluent release over 50 years of a person's lifetime. However, most of the dose commitment calculated here is delivered in the first year, so the comparison is reasonably valid.

For comparison purposes, the doses in the site summary tables are given to two significant figures; however, the data and models used to calculate the doses limit their accuracy to at most one significant figure. 


\section{SITE COMPARISONS}

Table 5 shows the dose commitments for the past 14 years along with the energy produced in that year. The last column shows the cost/benefit for commercial nuclear power as a ratio of population dose to generated power. Except for 1979 (the year of the TMI-2 accident) this ratio has been tending downward even though the population around many of the sites has been rising.

TABLE 5. Comparison of Annual Population Dose Commitments and Energy Output for the Past 14 Years

\begin{tabular}{|c|c|c|c|c|c|}
\hline Year & Liquid & $\begin{array}{l}\text { Commi } \\
\text { Air } \\
\text { rson-r }\end{array}$ & $\begin{array}{l}\text { ts } \\
\text { Total }\end{array}$ & $\begin{array}{l}\text { Energy } \\
\text { Output } \\
(T W-h r)\end{array}$ & $\begin{array}{l}\text { Cost/Benefit } \\
\text { (person-rem } \\
\text { TW-hr) }\end{array}$ \\
\hline $\begin{array}{l}1975 \\
1976 \\
1977 \\
1978 \\
1979\end{array}$ & $\begin{array}{r}76 \\
82 \\
160 \\
110 \\
220\end{array}$ & $\begin{array}{r}1300 \\
390 \\
540 \\
530 \\
1600\end{array}$ & $\begin{array}{r}1300 \\
470 \\
700 \\
640 \\
1800\end{array}$ & $\begin{array}{l}170 \\
180 \\
250 \\
270 \\
250\end{array}$ & $\begin{array}{l}7.6 \\
2.5 \\
2.8 \\
2.3 \\
7.2\end{array}$ \\
\hline $\begin{array}{l}1980 \\
1981 \\
1982 \\
1983 \\
1984\end{array}$ & $\begin{array}{r}120 \\
87 \\
50 \\
95 \\
160\end{array}$ & $\begin{array}{r}57 \\
63 \\
87 \\
76 \\
120\end{array}$ & $\begin{array}{l}180 \\
150 \\
140 \\
170 \\
280\end{array}$ & $\begin{array}{l}250 \\
280 \\
270 \\
280 \\
320\end{array}$ & $\begin{array}{l}0.73 \\
0.54 \\
0.51 \\
0.60 \\
0.87\end{array}$ \\
\hline $\begin{array}{l}1985 \\
1986 \\
1987 \\
1988\end{array}$ & $\begin{array}{l}91 \\
71 \\
56 \\
65\end{array}$ & $\begin{array}{r}110 \\
44 \\
22 \\
10\end{array}$ & $\begin{array}{r}200 \\
110 \\
78 \\
75\end{array}$ & $\begin{array}{l}370 \\
410 \\
450 \\
510\end{array}$ & $\begin{array}{l}0.54 \\
0.27 \\
0.17 \\
0.15\end{array}$ \\
\hline
\end{tabular}

The reactor sites are compared in Table 6 as to the total population dose over the years of this study, 1975-1988. The sites were placed within six groupings by person-rem depending on resulting population dose summed over each of the years through 1988:

$\begin{array}{ll}\text { I } & \text { Greater than } 100 \\ \text { II } & 31-100 \\ \text { III } & 11-30 \\ \text { IV } & 3.1-10 \\ \text { V } & 1-3 \\ \text { VI } & \text { Less than } 1\end{array}$


Table 6 shows the sites within the groups along with the reactor manufacturer, year of commercial operation commencement, and the indicated population doses in person-rem. The manufacturer codes are as follows:

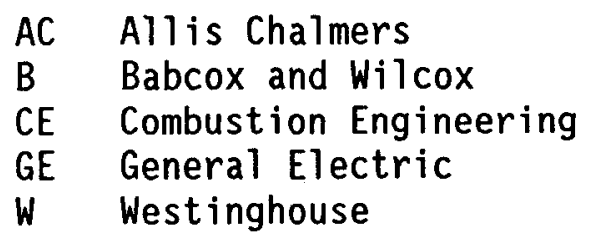

Table 7 shows the average population dose in person-rem for the last three years. The sites are listed in order of average dose with the high doses toward the top. Breaks in the table are indicated every half order of magnitude: $0.03, .01 .03$, etc.

Table 8 shows the present contributions of all radionuclides contributing over $3 \%$ to the population dose from 1988 effluents. The contributors are shown for each site for both liquid (L) and air (A) pathways. Note that only a few nuclides of those reported by the sites (Tichler, et al. 1991) usually contribute to the dose. 


\title{
TABLE 6. Total-Body Population Doses from Nuclear, Power Plant Effluents During Normal Operations, (a) (b) 1975-1988
}

rear

\author{
.
}

commerclat

Site

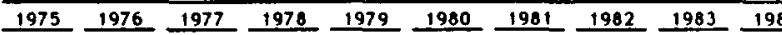

\section{1. $>100$ person-rea}

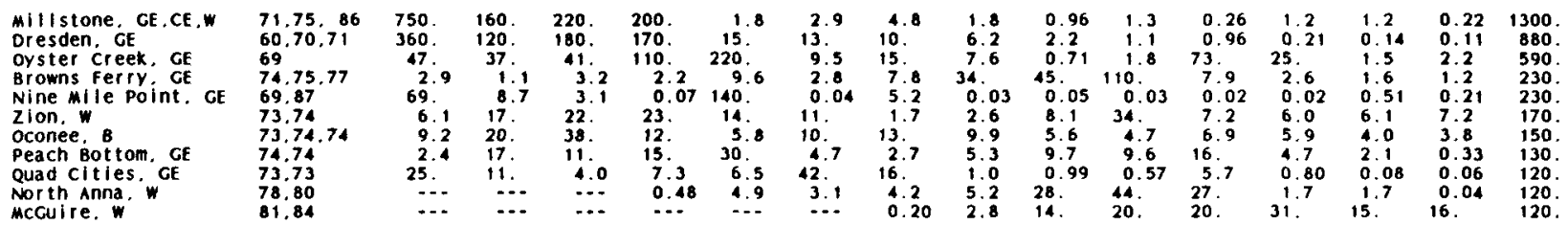

11. 31 - 100 person-rea

Pilgrim. GE

Cook.w 72.78

Indian point

Hatch. GE

Lacrosse. $A C$

Big Rock Point, GE

Crystal River.

Arkansas One, B
Rancho Seco, B

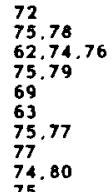

111. $11-30$ person-rem

Kewaunee.

$\begin{array}{ll}\text { Haddam Neck. W } & 74 \\ \text { Surry } & 68\end{array}$

$\begin{array}{ll}\text { Surry. W } & 72 \\ \text { Davis-8esse, B } & 77 \\ \text { Humboldt Bay, GE } & 73 \\ \text { Fitzpatrick, GE } & 73\end{array}$

$\begin{array}{ll}\text { Sumer, } & 82 \\ \text { Calvert Cliffs. CE } \quad 75.77\end{array}$

san onofre. $w$. 68.83 .84

$\begin{array}{ll}\text { Robinson. W } & 71 \\ \text { Turkey Point. w } & \mathbf{7 2 . 7 3}\end{array}$

Salem,
Three mile island, B 74.81
St. Lucie. CE

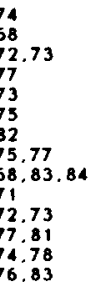

\begin{tabular}{clcl}
8.5 & 5.1 & 1.9 & 0.5 \\
0.54 & 3.7 & 2.4 & 5.6 \\
5.4 & 3.4 & 3.7 & 1.4 \\
\hdashline.- &..- & $14 . .2$ & 0.60 \\
18. & 5.8 &.- .56 &..- \\
0.09 & 1.4 & 0.56 & 0.37 \\
\hdashline 0.50 & 0.74 & 1.9 & 2.6 \\
0.28 & 1.4 & 0.78 & 1.8 \\
9.3 & 0.28 & 0.47 & 0.45 \\
0.22 & 0.28 & 0.40 & 9.1 \\
\hdashline-5 &..- & 0.12 & 0.37 \\
0.57 & 1.4 & 2.0 & 2.2 \\
..- & 0.03 & 0.65 & 1.1
\end{tabular}

\begin{tabular}{lcc}
6.2 & 14. & 52. \\
0.21 & 5.0 & 23. \\
3.8 & 9.1 & 13. \\
\hdashline. .0 & 0.03 & 35. \\
7.0 & 12. & 9.4 \\
4.6 & 7.7 & 2.6 \\
0.02 & 0.53 & 6.4 \\
\hdashline. .58 &.- .5 & 0.02 \\
0.38 & 4.5 & 1.6 \\
0.05 & 0.01 & 0.06
\end{tabular}

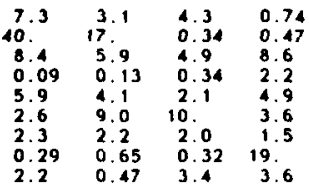

$\begin{array}{lll}2.3 & 4.1 & 0.05 \\ 0.72 & 0.21 & 0.34 \\ 5.5 & 4.6 & 3.2 \\ 2.8 & 5.8 & 8.0 \\ 7.0 & 1.8 & 1 . \\ 1.2 & 0.57 & 4.5 \\ 14.03 & 10 . & 4 . \\ 0.03 & 4.8 & 6.8 \\ 4.5 & 3.6 & 3.8\end{array}$

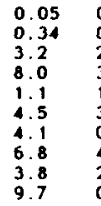

$\begin{array}{lllll}0.56 & 0.02 & 0.01 & 0.01 & 95 \\ 0.54 & 0.17 & 0.62 & 0.22 & 89 . \\ 2.8 & 3.2 & 6.2 & 0.43 & 80 . \\ 3.4 & 2.8 & 5.0 & 6.4 & 72 . \\ 1.0 & 0.81 & 0.82 & 0.41 & 58 . \\ 3.3 & 2.0 & 0.74 & 0.85 & 53 . \\ 0.18 & 0.71 & 0.62 & 0.04 & 45 . \\ 4.4 & 2.4 & 0.72 & 0.20 & 40 . \\ 2.0 & 4.7 & 1.7 & 1.0 & 38 . \\ 0.25 & 0.02 & 0.01 & 0.08 & 32 .\end{array}$

1V. 3.1 - 10 person-rea
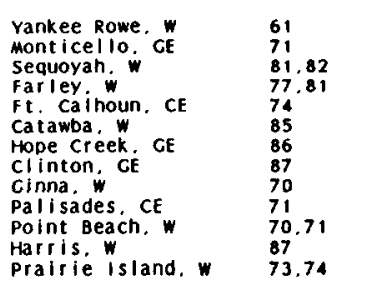

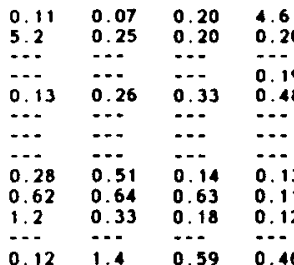
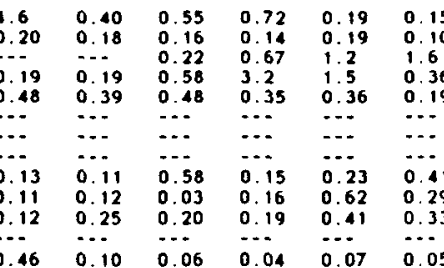

$\begin{array}{ll}0.15 & 0.48 \\ 10 & 0.05 \\ 16 & 1.8 \\ .36 & 0.23 \\ 19 & 1.9 \\ \cdots & -. . \\ \cdots & \cdots . . \\ .11 & 0.69 \\ .29 & 0.12 \\ .33 & 0.24 \\ .05 & 0.06\end{array}$
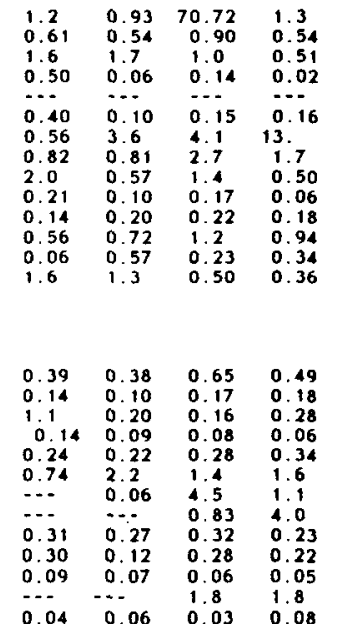

30.
29.
29.
28.
24.
24.
22.
21.
20.
13.
12.
11.
11.
10.

v. 1 - 3 per son-re

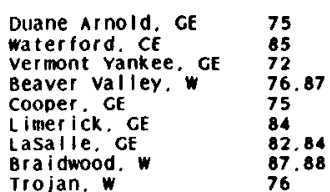

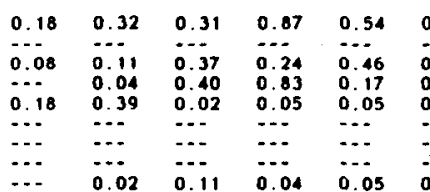

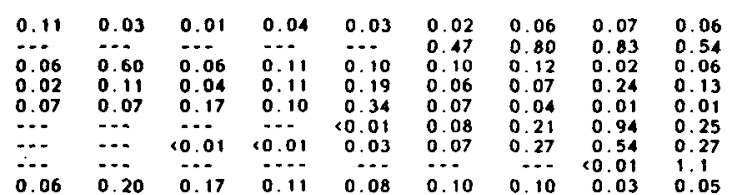

$\begin{array}{ll}0.06 & 2.7 \\ 0.54 & 2.6 \\ 0.06 & 2.5 \\ 0.13 & 2.4 \\ 0.01 & 1.6 \\ 0.25 & 1.5 \\ 0.27 & 1.2 \\ 1.1 & 1.1 \\ 0.05 & 1.1\end{array}$

vi. $C$ derson-rea

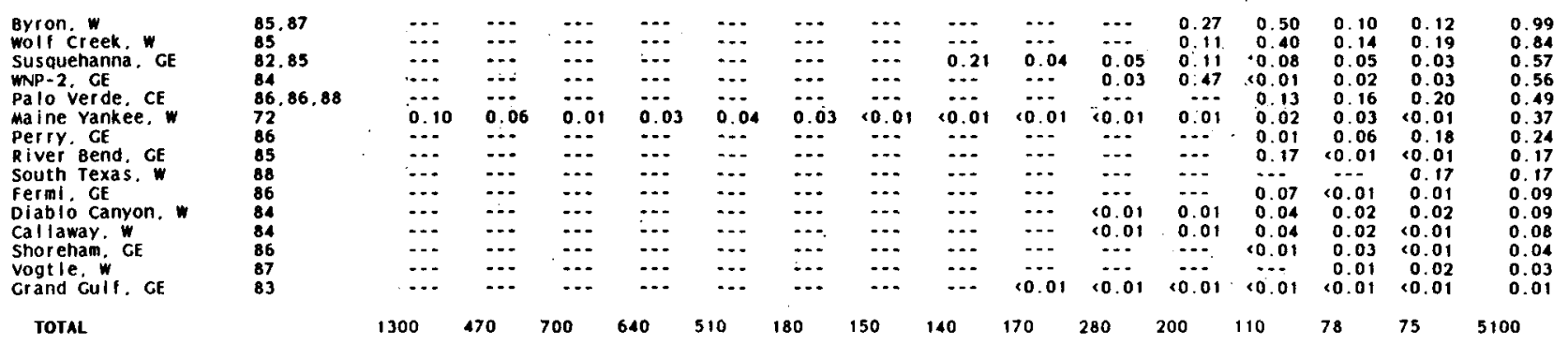

(a) Only the doses from the TMI accident, 1979 . are excluded.
(b) Does not include doses from nuclides not reported in releases, such as carbon-14. 
Table 7. Average Population Dose for Last Three Years, person-rem

\begin{tabular}{|c|c|c|c|c|}
\hline site & 1986 & 1987 & 1988 & Average \\
\hline McGuire & 31.10 & 15.17 & 16.17 & 20.8 \\
\hline $\begin{array}{l}\text { Oyster Creek } \\
\text { Summer } \\
\text { Zion } \\
\text { Hatch } \\
\text { Oconee } \\
\text { Indian Pt. }\end{array}$ & $\begin{array}{r}25.00 \\
3.60 \\
6.04 \\
2.75 \\
5.93 \\
3.20\end{array}$ & $\begin{array}{l}1.50 \\
4.11 \\
6.09 \\
4.96 \\
3.97 \\
6.20\end{array}$ & $\begin{array}{r}2.20 \\
13.01 \\
7.24 \\
6.43 \\
3.84 \\
0.43\end{array}$ & $\begin{array}{l}9.57 \\
6.91 \\
6.46 \\
4.71 \\
4.58 \\
3.28\end{array}$ \\
\hline $\begin{array}{l}\text { Arkansas One } \\
\text { Peach Bottom } \\
\text { Hope Creek } \\
\text { Browns Ferry } \\
\text { Calvert Cliffs } \\
\text { Catawba } \\
\text { Clinton } \\
\text { Harris } \\
\text { Big Rock Point } \\
\text { North Anna } \\
\text { Crystal River } \\
\text { Surry }\end{array}$ & $\begin{array}{l}4.73 \\
4.71 \\
0.06 \\
2.64 \\
0.81 \\
2.15 \\
-. . \\
-. . \\
1.97 \\
1.67 \\
2.41 \\
1.70\end{array}$ & $\begin{array}{l}1.71 \\
2.07 \\
4.50 \\
1.60 \\
2.66 \\
1.37 \\
0.83 \\
1.78 \\
0.74 \\
1.72 \\
0.72 \\
1.04\end{array}$ & $\begin{array}{l}1.03 \\
0.33 \\
1.09 \\
1.20 \\
1.74 \\
1.61 \\
4.01 \\
1.80 \\
0.85 \\
0.04 \\
0.20 \\
0.51\end{array}$ & $\begin{array}{l}2.49 \\
2.37 \\
1.88 \\
1.81 \\
1.74 \\
1.71 \\
1.61 \\
1.19 \\
1.19 \\
1.14 \\
1.11 \\
1.08\end{array}$ \\
\hline $\begin{array}{l}\text { Kewaunee } \\
\text { Salem } \\
\text { Millstone } \\
\text { San Onofre } \\
\text { Waterford } \\
\text { St. Lucie } \\
\text { LaCrosse } \\
\text { Haddam Neck } \\
\text { Yankee Rowe } \\
\text { Línerick } \\
\text { Brunswick } \\
\text { Three Mile Isl. } \\
\text { Braidwood } \\
\text { LaSalle } \\
\text { Cook } \\
\text { Quad cities }\end{array}$ & $\begin{array}{l}0.93 \\
0.72 \\
1.23 \\
0.57 \\
0.80 \\
1.30 \\
0.81 \\
0.54 \\
0.38 \\
0.21 \\
0.71 \\
0.57 \\
0.27 \\
0.27 \\
0.17 \\
0.80\end{array}$ & $\begin{array}{r}0.72 \\
1.17 \\
1.20 \\
1.43 \\
0.83 \\
0.50 \\
0.82 \\
0.90 \\
0.65 \\
0.94 \\
0.62 \\
0.23 \\
<0.01 \\
0.54 \\
0.62 \\
0.08\end{array}$ & $\begin{array}{l}1.30 \\
0.94 \\
0.22 \\
0.50 \\
0.54 \\
0.36 \\
0.41 \\
0.54 \\
0.49 \\
0.25 \\
0.04 \\
0.34 \\
1.11 \\
0.27 \\
0.22 \\
0.06\end{array}$ & $\begin{array}{l}0.98 \\
0.94 \\
0.89 \\
0.83 \\
0.72 \\
0.72 \\
0.68 \\
0.66 \\
0.50 \\
0.47 \\
0.46 \\
0.38 \\
0.37 \\
0.36 \\
0.33 \\
0.31\end{array}$ \\
\hline $\begin{array}{l}\text { Ft. Calhoun } \\
\text { Ginna } \\
\text { Nine Mile Point } \\
\text { Wolf Creek } \\
\text { Byron } \\
\text { Sequoyah } \\
\text { Pal isades } \\
\text { Turkey Point } \\
\text { Palo Verde } \\
\text { Dresden } \\
\text { Mont icello } \\
\text { Beaver Vall ey } \\
\text { Fitzpatrick } \\
\text { Robinson }\end{array}$ & $\begin{array}{l}0.22 \\
0.27 \\
0.02 \\
0.40 \\
0.50 \\
0.20 \\
0.12 \\
0.20 \\
0.13 \\
0.21 \\
0.10 \\
0.07 \\
0.10 \\
0.10\end{array}$ & $\begin{array}{l}0.28 \\
0.32 \\
0.51 \\
0.14 \\
0.10 \\
0.16 \\
0.28 \\
0.22 \\
0.16 \\
0.14 \\
0.17 \\
0.23 \\
0.15 \\
0.17\end{array}$ & $\begin{array}{l}0.34 \\
0.23 \\
0.21 \\
0.19 \\
0.12 \\
0.28 \\
0.21 \\
0.18 \\
0.20 \\
0.11 \\
0.18 \\
0.13 \\
0.16 \\
0.06\end{array}$ & $\begin{array}{l}0.28 \\
0.28 \\
0.25 \\
0.24 \\
0.24 \\
0.21 \\
0.20 \\
0.20 \\
0.16 \\
0.15 \\
0.15 \\
0.15 \\
0.14 \\
0.11\end{array}$ \\
\hline $\begin{array}{l}\text { Perry } \\
\text { Farley } \\
\text { Davis-Besse } \\
\text { Duane Arnold } \\
\text { Point Beach } \\
\text { Trojan } \\
\text { Vermont Yankee } \\
\text { River Bend } \\
\text { South Texas } \\
\text { Prairie Island } \\
\text { Susquehanna } \\
\text { Rancho Seco }\end{array}$ & $\begin{array}{l}0.00 \\
0.09 \\
0.06 \\
0.06 \\
0.07 \\
0.10 \\
0.12 \\
0.17 \\
-1 . \\
0.06 \\
0.08 \\
0.02\end{array}$ & $\begin{array}{l}0.06 \\
0.08 \\
0.14 \\
0.07 \\
0.06 \\
0.03 \\
0.02 \\
<0.01 \\
-. .03 \\
0.03 \\
0.05 \\
0.01\end{array}$ & $\begin{array}{l}0.18 \\
0.06 \\
0.02 \\
0.06 \\
0.05 \\
0.05 \\
0.06 \\
<0.01 \\
0.17 \\
0.08 \\
0.03 \\
0.08\end{array}$ & $\begin{array}{l}0.08 \\
0.08 \\
0.07 \\
0.06 \\
0.06 \\
0.06 \\
0.06 \\
0.06 \\
0.06 \\
0.06 \\
0.06 \\
0.04\end{array}$ \\
\hline $\begin{array}{l}\text { Fermi } \\
\text { Diablo Canyon } \\
\text { Callaway } \\
\text { Maine Yankee } \\
\text { Cooper } \\
\text { UNP-2 } \\
\text { Shoreham } \\
\text { Pilgrim } \\
\text { Vogtle } \\
\text { Grand Gulf }\end{array}$ & $\begin{array}{r}0.07 \\
0.04 \\
0.04 \\
0.02 \\
0.04 \\
<0.01 \\
<0.01 \\
0.02 \\
-0.01\end{array}$ & $\begin{array}{r}<0.01 \\
0.02 \\
0.02 \\
0.03 \\
0.01 \\
0.02 \\
0.03 \\
0.01 \\
0.01 \\
<0.01\end{array}$ & $\begin{array}{r}0.01 \\
0.02 \\
<0.01 \\
<0.01 \\
0.01 \\
0.03 \\
<0.01 \\
<0.01 \\
0.02 \\
<0.01\end{array}$ & $\begin{array}{l}0.03 \\
0.03 \\
0.02 \\
0.02 \\
0.02 \\
0.02 \\
0.01 \\
0.01 \\
0.01 \\
0.01\end{array}$ \\
\hline
\end{tabular}


TABLE 8. Major Radionuclide Contributions to Population Doses from Liquid (L) and Air (A) Pathways

\begin{tabular}{|c|c|c|c|c|c|c|c|c|c|c|c|c|c|c|c|c|c|c|c|c|c|c|c|}
\hline \multirow[b]{2}{*}{ site } & \multirow[b]{2}{*}{ Path } & \multirow[b]{2}{*}{$\begin{array}{l}\text { Dose } \\
\text { (Person } \\
\text { rem) }\end{array}$} & \multicolumn{8}{|c|}{ major } & \multicolumn{4}{|c|}{ contributers } & \multicolumn{3}{|c|}{ (Percent): } & \multirow[b]{2}{*}{$\begin{array}{l}1 \\
1 \\
3 \\
1\end{array}$} & \multirow[b]{2}{*}{$\begin{array}{l}\text { C5 } \\
1 \\
3 \\
4\end{array}$} & \multirow[b]{2}{*}{$\begin{array}{l}C s \\
1 \\
3 \\
7 \\
+D \\
\end{array}$} & \multirow[b]{2}{*}{$\begin{array}{l}x e \\
1 \\
3 \\
3\end{array}$} & \multirow[b]{2}{*}{$\begin{array}{l}x e \\
1 \\
3 \\
5\end{array}$} & \multirow[b]{2}{*}{$\begin{array}{l}\text { Xe } \\
1 \\
3 \\
8 \\
+D \\
\end{array}$} \\
\hline & & & $\underset{3}{H}$ & $\begin{array}{l}c \\
1 \\
4\end{array}$ & $\begin{array}{l}\mathrm{Na} \\
2 \\
4\end{array}$ & $\frac{A r}{1}$ & $\begin{array}{l}\text { Mn } \\
5 \\
4\end{array}$ & $\begin{array}{l}\mathrm{Fe} \\
5 \\
5\end{array}$ & $\begin{array}{l}\mathrm{Fe} \\
5 \\
9\end{array}$ & $\begin{array}{l}\text { co } \\
5 \\
8\end{array}$ & $\begin{array}{l}\text { co } \\
6 \\
0\end{array}$ & $\begin{array}{l}\text { Zn } \\
6 \\
5 \\
0\end{array}$ & $\begin{array}{l}\mathrm{Kr} \\
8 \\
5\end{array}$ & $\begin{array}{l}K r \\
8 \\
7 \\
+D\end{array}$ & $\begin{array}{l}K r \\
8 \\
8 \\
+D\end{array}$ & $\begin{array}{l}\mathbf{S} r \\
8 \\
9\end{array}$ & $\begin{array}{l}\text { Sr } \\
9 \\
0\end{array}$ & & & & & & \\
\hline ARKANSAS ONE & L & $\begin{array}{l}1.0 \\
0.034\end{array}$ & & & & & & & & & & & & & & & & & 34 & 65 & 53 & 44 & \\
\hline BEAVER VALLeY & A & $\begin{array}{l}0.046 \\
0.085\end{array}$ & 99 & & & & & & & & & & & & & & & & & & 3 & & \\
\hline BIG ROCK POINT & A & $\begin{array}{l}0.75 \\
0.10\end{array}$ & & & & & & & & & & & & 21 & 48 & & 3 & & 14 & 81 & & 15 & 13 \\
\hline BRAIDWOOOO & A & $\begin{array}{r}1 \\
0.0061 \\
\end{array}$ & 49 & & & 6 & 3 & & & 18 & & & & & & & & & 20 & 56 & 42. & & \\
\hline BROWNS FERRY & A. & $\begin{array}{l}1.2 \\
0.0011 \\
.9 .1 .\end{array}$ & 15 & & & & & & & & 74 & & & & & & & & 29 & $\begin{array}{r}71 \\
9 \\
-1\end{array}$ & & & \\
\hline BRUNSWICK & A & $\begin{array}{l}0.0047 \\
0.035 \\
\end{array}$ & & & & 3 & 4 & 10 & 25 & & $\begin{array}{r}35 \\
6 \\
\end{array}$ & & & & 26 & & & & 8 & 16 & 6 & 50 & \\
\hline BYRON & A & $\begin{array}{l}0.092 \\
0.029 \\
\end{array}$ & & & & & & & & & & & & & & & & & 57 & 41 & 90 & 6 & \\
\hline Caliaway & $\therefore$ & $\begin{array}{l}0.0001 \\
0.0065 \\
\end{array}$ & 30 & & & & & & & & & & & & & & & & 50 & 47 & 51. & 17 & \\
\hline CALVERT CL IfFS & A & $\begin{array}{l}1.5 \\
0.24 \\
\end{array}$ & 8 & & & & & & & 4 & & & & & & & & & 41 & $\begin{array}{r}51 \\
7 \\
\end{array}$ & 67. & $\begin{array}{r}15 \\
-.-\end{array}$ & \\
\hline CATAWBA & A. & $\begin{array}{l}1.4 \\
0.21\end{array}$ & 19 & & & 3 & & & & & & & & & & & & & 50 & 47 & $\begin{array}{r}71 \\
-. .2\end{array}$ & 5 & \\
\hline CL. INTON & L & $\begin{array}{l}4.0 \\
0.0087 \\
0.0 .6\end{array}$ & $\begin{array}{r}3 \\
68 \\
-\end{array}$ & & & & $\begin{array}{r}45 \\
5 \\
\ldots .\end{array}$ & & & 6 & $\begin{array}{r}15 \\
18 \\
.2\end{array}$ & & & & & 3 & 22 & & . & 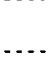 & 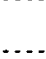 & $\therefore$ & \\
\hline cook & L & $\begin{array}{l}0.21 \\
0.0059 \\
-.2 .0\end{array}$ & $\begin{array}{l}54 \\
18 \\
-\end{array}$ & & & & & & & & & & & & 7 & & & & $\begin{array}{r}26 \\
11 \\
-\end{array}$ & $\begin{array}{l}20 \\
14\end{array}$ & 37 & 9 & \\
\hline COOPER & i & $\begin{array}{r}0.0068 \\
0.0049 \\
\end{array}$ & & & & & & & & & & & & & 50 & & & & 49 & 50 & 8 & 27 & \\
\hline CRYSTAL RIVER & L & $\begin{array}{l}0.19 \\
0.011\end{array}$ & $\begin{array}{r}17 \\
6 \\
6\end{array}$ & & & & & 20 & & 10 & 28 & & & & & & & & 9 & $\begin{array}{l}12 \\
\cdots\end{array}$ & 81 & 12 & \\
\hline DAVIS-BESSE & $\therefore$ & $\begin{array}{l}0.0018 \\
0.016 \\
\end{array}$ & 87 & & & & & & & & 4 & & & & 3 & & & & 30 & 48 & 9 & & \\
\hline DIABLO CANYON & $\therefore$ & $\begin{array}{l}0.0063 \\
0.015 \\
\end{array}$ & 76 & & & & & 22 & & 6 & 4 & & & & & & & & 37 & 25 & 18 & 4 & \\
\hline DRESDEN & i & 0.11 & 27 & & & & & & & & 15 & & & & & & & 3 & & & & 47 & \\
\hline DUANE ARNOLD & L & $\begin{array}{l}0 \\
0.064 \\
-\end{array}$ & 10 & & & & & & & & 5 & & & 7 & 26 & & & & & & 4 & $\begin{array}{r}45 \\
-.-\end{array}$ & \\
\hline FARLEY & i & $\begin{array}{l}0.020 \\
0.045 \\
0\end{array}$ & $\begin{array}{r}12 \\
74 \\
.2 .\end{array}$ & & & 8 & & & & & & & & & & & & & 24 & 62 & 6 & 9 & \\
\hline FERMI & i & $\begin{array}{r}0.010 \\
0.0024 \\
0.8\end{array}$ & 3 & & 19 & & $\begin{array}{l}4 \\
3\end{array}$ & & & 3 & 6 & $\begin{array}{r}86 \\
8 \\
-\end{array}$ & 3. & & & & 36 & & & & & 19 & \\
\hline FITZPATRICK & A & $\begin{array}{l}0.017 \\
0.14 \\
0.1 .\end{array}$ & 4 & & & & & & & & & & 4 & 5 & 59 & & & & 39 & $\begin{array}{l}56 \\
\cdots\end{array}$ & 6 & 23 & \\
\hline FORT CALHOUN & A & $\begin{array}{l}0.33 \\
0.0080 \\
0.0\end{array}$ & $\begin{array}{r}64 \\
9 \\
\cdots\end{array}$ & & & 3 & & & & & & & & & & & & & 13 & 21 & 86 & & \\
\hline CINNA & i & $\begin{array}{l}0.16 \\
0.073 \\
-0.1\end{array}$ & $\begin{array}{r}80 \\
70 \\
-70 \\
\end{array}$ & 27. & & & & & & & & & & & & & & & 9 & 11 & & 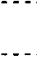 & \\
\hline GRAND GULF & A & $\begin{array}{l}0.0004 \\
0.0007\end{array}$ & 64 & & & 11 & 17 & & & & 3 & & & $\therefore$ & 12. & & & & 50 & $\begin{array}{l}28 \\
\ldots .\end{array}$ & & 5 & \\
\hline MADOAM NECK & L & $\begin{array}{l}0.077 \\
0.46 \\
0.2\end{array}$ & 34 & & & & & & & & & & & & & & & & 33 & 63 & 57 & 5 & \\
\hline HARRIS & L & $\begin{array}{l}1.7 \\
0.10\end{array}$ & 85 & & & & & & & & & & & & 6 & & & & & 12 & 83 & 9 & \\
\hline E. I. Match & A & $\begin{array}{r}6.4 \\
0.035 \\
\end{array}$ & 19 & & & & & & & & & & & & 15 & & & & 52 & 47 & 44 & $\begin{array}{r}13 \\
-.-\end{array}$ & \\
\hline HOPE CREEK & A & $\begin{array}{l}0.96 \\
0.13 \\
\end{array}$ & 93 & & & & & & 4 & & 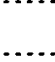 & 95 & & & $\therefore$ & & & & & & & & \\
\hline INDIAN POINT & A. & $\begin{array}{l}0.092 \\
0.34\end{array}$ & 9 & & & & & & & & 17 & & & & & & & & 40 & $\begin{array}{r}57 \\
3\end{array}$ & 56 & 11 & \\
\hline KEWAUNEE & A & $\begin{array}{l}1.3 \\
0.0022\end{array}$ & $\begin{array}{r}55 \\
95 \\
-\end{array}$ & & & & & & & & & & & & & & & & 24 & $\begin{array}{c}19 \\
\ldots .\end{array}$ & $\ldots$ & & \\
\hline LACrosse & A & 0.41103 & 99 & & & & & & & & & & & & & & & & 4 & 95 & & & \\
\hline LASALLe & i & $\begin{array}{l}0.27 \\
0.27\end{array}$ & & & & & & & & & & & 5 & & 80 & & & & & & 9 & 4 & \\
\hline LIMERICK & L & . & 71 & & & & & & & & $=-$ & & & & & & & & & & 9 & 0 & \\
\hline MAINE Yankee & A & $\begin{array}{l}0.0029 \\
0.0047 \\
0.06\end{array}$ & $\begin{array}{r}8 \\
66 \\
-6\end{array}$ & & & & & 36 & & 8 & 19 & & & & & & & & 6 & 21 & 29 & & \\
\hline MCOUIRE & A & $\begin{array}{r}16.17 \\
0.17\end{array}$ & $\begin{array}{l}86 \\
13 \\
\end{array}$ & & & & & & & & 3 & & & & 4 & & & & 3 & 4 & 65 & 13 & \\
\hline
\end{tabular}


IABLE 8. (Contd)

\begin{tabular}{|c|c|c|c|c|c|c|c|c|c|c|c|c|c|c|c|c|c|c|c|}
\hline & & & & & & & & maj & & con & ntribut & ters & (Perc) & cent) & & & & & \\
\hline site & Path & $\begin{array}{l}\text { Dose } \\
\text { (Person } \\
\text { rem) }\end{array}$ & $\stackrel{H}{3}$ & $\begin{array}{ll}\mathrm{C} & \mathrm{Na} \\
1 & 2 \\
4 & 4\end{array}$ & $\hat{a}_{1}^{r}$ & $\begin{array}{l}m_{n} \\
5 \\
4\end{array}$ & $\begin{array}{l}\mathrm{Fe} \\
5 \\
5\end{array}$ & $\begin{array}{l}\mathrm{Fe} \\
5 \\
9\end{array}$ & $\begin{array}{l}\text { co } \\
5 \\
8\end{array}$ & $\begin{array}{l}\text { co } \\
6 \\
0\end{array}$ & $\begin{array}{ll}\mathrm{Zn} & \mathrm{kr} \\
\mathbf{6} & 8 \\
\mathbf{5} & 5 \\
& \mathbf{m}\end{array}$ & $\begin{array}{l}k r \\
8 \\
7 \\
7\end{array}$ & $\begin{array}{cc}\mathrm{Kr} & \mathrm{S} \\
\mathrm{g} & 8 \\
8 & 8 \\
+\mathrm{D} & 9 \\
\end{array}$ & $\begin{array}{cc}S r & S r \\
8 & 9 \\
9 & 0 \\
& +0 \\
\end{array}$ & $\begin{array}{ll}r & 1 \\
1 & 1 \\
0 & 3 \\
0 & 1 \\
\end{array}$ & $\begin{array}{l}C S \\
1 \\
3 \\
4 \\
+D \\
\end{array}$ & $\begin{array}{l}C S \\
1 \\
3 \\
7 \\
+D \\
\end{array}$ & $\begin{array}{l}x \mathbf{x} \\
1 \\
3 \\
3 \\
\end{array}$ & $\begin{array}{ll}\text { Xe } & \text { xe } \\
1 & 1 \\
3 & 3 \\
5 & 8 \\
5 & 8 \\
+D & +0\end{array}$ \\
\hline MILLSTONE & L & $\begin{array}{l}0.0027 \\
0.22\end{array}$ & .47 & & & & 12 & 5 & 18 & 38 & 8 & 6 & & & & 6 & 10 & 15 & $\begin{array}{r}15 \quad 12 \\
.\end{array}$ \\
\hline MONTICELLO & L & $\begin{array}{l}0 \\
0.18\end{array}$ & 21. & & & & & & & & & & 16 & & & & & 22 & $5 \quad 25$ \\
\hline NINE MILE POINT & I & $\begin{array}{l}0.21 \\
0.0044\end{array}$ & $\begin{array}{c}8 \\
44\end{array}$ & & 8 & 5 & & 8 & 13 & $\begin{array}{r}20 \\
25\end{array}$ & 46 & & & & & & 9 & & 3 \\
\hline NORTH ANNA & A & $\begin{array}{l}0.01 \\
0.026 \\
0.026\end{array}$ & 77 & & & & & & & & & & & & & 40 & 56 & 18 & . \\
\hline OCONEE & $\therefore$. & $\begin{array}{l}3.3 \\
0.54 \\
-. .2\end{array}$ & $\begin{array}{c}47 \\
\ldots \\
\ldots\end{array}$ & & & & & & & & & & & & & 22 & 29 & 89 & 4. \\
\hline OYSTER CREEK & A & $\begin{array}{l}0.20 \\
2.00\end{array}$ & 4 & & & & & & & 61 & & 15. & 57 & & & 4 & 27 & & 23. \\
\hline PAL ISADES & A. & $\begin{array}{l}0.19 \\
0.025 \\
0.02\end{array}$ & $\begin{array}{r}8 \\
3 \\
-\ldots\end{array}$ & & & & & & & & & & 3 & & & 4 & 86 & 89 & \\
\hline PALO VERDE & L & $\begin{array}{l}0 \\
0.20 \\
\end{array}$ & 74 & & & & & & & & & & & & & & & 22. & 3. \\
\hline PEACH BOTTOM & L & $\begin{array}{l}0.32 \\
0.014 \\
0\end{array}$ & $\begin{array}{r}7 \\
86 \\
\end{array}$ & & & & & & & 5 & & & & & 8 & 41 & 42 & & \\
\hline PERRY & A & $\begin{array}{l}0.11 \\
0.068\end{array}$ & $\begin{array}{r}65 \\
3 \\
-3\end{array}$ & & & 3 & & 3 & & 12 & & 3 & 10 & 10 & $\ddot{0}$ & 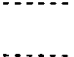 & 3 & 36 & 44 \\
\hline PILGR IM & A & $\begin{array}{l}0.0014 \\
0.0006 \\
\end{array}$ & 11 & & & & 18 & & & $\begin{array}{l}38 \\
83 \\
-. .\end{array}$ & & & & & & & 41 & & \\
\hline POINT BEACH & A & $\begin{array}{l}0.017 \\
0.030 \\
. \quad . .\end{array}$ & $\begin{array}{c}84 \\
94 .\end{array}$ & & & & & & & & & & & & & & 12 & & \\
\hline PRAIRIE ISLAND & L & $\begin{array}{l}0.0031 \\
0.077\end{array}$ & $\begin{array}{l}68 \\
, 99\end{array}$ & & & & 18 & & & 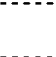 & & & 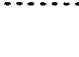 & & 6 & - & 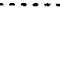 & & \\
\hline QUAD CITIES & A & $\begin{array}{r}0.040 \\
0.016 \\
0.016 \\
\end{array}$ & $\begin{array}{r}5 \\
67 \\
-67\end{array}$ & & & & & & & 22 & & & 5. & 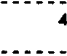 & $\because$ & 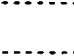 & 87 & & \\
\hline RANCHO SECO & A & $\begin{array}{r}0.021 \\
0.059 \\
\end{array}$ & 14 & & & & & & & & & & 4 & & & 21 & 77 & & 8 \\
\hline RIVER BEND & A & $\begin{array}{l}0.007 \\
0.0010\end{array}$ & 74 & & & 14 & & & & 15 & 65 & & & & & 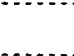 & 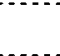 & & 19 \\
\hline ROBINSON & $\therefore$ & $\begin{array}{l}0.041 \\
0.020 \\
0.02\end{array}$ & 10 & & & & & & & & & & & & & 37 & 54 & 63 & 22 \\
\hline SAINT LUCIE & A & $\begin{array}{l}0.0002 \\
0.36 \\
-36\end{array}$ & $\begin{array}{r}10 \\
16 \\
-\end{array}$ & & & & 26 & & 7 & 17 & & 6 & & & & 19 & 18 & 44 & 32. \\
\hline SALEM & A & $\begin{array}{l}0.28 \\
0.66\end{array}$ & 84. & & & & 34 & & 22 & 15 & & & & & & 14 & 9 & 12. & \\
\hline SAN ONOFRE & A & $\begin{array}{l}0.023 \\
0.48 \\
0.9\end{array}$ & $\begin{array}{c}9 \\
5 \\
5\end{array}$ & & & & 3 & & 4 & & & & & & & 44 & 36 & 67 & 21. \\
\hline SEQUOYAH & A. & $\begin{array}{l}0.26 \\
0.019\end{array}$ & $\begin{array}{l}40 \\
\therefore 2 \\
\therefore . .\end{array}$ & & & & & & & & & & & & & 21 & 35 & 51 & 5 \\
\hline SHOREHAM & A. & $\begin{array}{l}0.0015 \\
0 .\end{array}$ & & & & & & & 100 & & & & & & & & & & \\
\hline SOUTH TEXAS & A & $\begin{array}{l}0.14 \\
0.028\end{array}$ & $\begin{array}{c}7 \\
3 \\
-1\end{array}$ & & $\begin{array}{c}96 \\
-9 .\end{array}$ & & & 23 & 65 & 5 & & & & & & & - & & \\
\hline SUMUER & L & $\begin{array}{l}13.007 \\
0.007\end{array}$ & $\begin{array}{c}5 \\
6 \\
6\end{array}$ & & & & & & & & & & 5 & & & 67 & 28 & 70 & \\
\hline SURRY & A. & $\begin{array}{l}0.47 \\
0.040 \\
\ldots . .6\end{array}$ & .44 & & & & 32 & 6 & 7 & $\begin{array}{c}26 \\
6 \\
-\end{array}$ & & & & & & $\begin{array}{r}9 \\
4 \\
-1\end{array}$ & $\begin{array}{r}20 \\
6 \\
\end{array}$ & 35 & \\
\hline SUSQUEHANNA & A. & $\begin{array}{r}0.018 \\
0.016 \\
0 .\end{array}$ & $\begin{array}{r}24 \\
81 \\
\therefore . .\end{array}$ & & & 3 & & & & & 4 & & & & & 43 & 24 & 17. & \\
\hline$T M I$ & A & $\begin{array}{l}0.12 \\
0.22\end{array}$ & $\begin{array}{r}77 \\
12 \\
.12\end{array}$ & & & & & & & & & & & 3 & 3 & 8 & 11 & 79 & \\
\hline TROJAN & A & $\begin{array}{l}0.0013 \\
0.045 \\
0.04\end{array}$ & $\begin{array}{r}16 \\
68 \\
-\end{array}$ & & & & & & & 3 & & & & & 8 & 28 & 42 & 28 & \\
\hline TURKEY POINT & $\begin{array}{l}\mathrm{t} \\
\mathrm{A}\end{array}$ & $\begin{array}{l}0.045 \\
0.14\end{array}$ & $\begin{array}{r}6 \\
60 \\
-.\end{array}$ & & 7. & & 27 & & & 51 & & & & & & 4 & 5 & 29 & \\
\hline VERMONT YANKEE & A & $\begin{array}{l}0 \\
0.059 \\
0\end{array}$ & $\begin{array}{r}96 \\
. .\end{array}$ & & & & & & & 3. & & & & & & & & & \\
\hline VOCTLE & A. & $\begin{array}{l}0.0029 \\
0.018\end{array}$ & $\begin{array}{l}18 \\
88 \\
.\end{array}$ & & 6 & 8 & 4 & 6 & 37 & 6 & 10 & & & & & & 11 & 5 & \\
\hline WATERFORD & A. & $\begin{array}{l}0.063 \\
0.48\end{array}$ & $\begin{array}{l}75 \\
37 \\
-\end{array}$ & & & & & & & & & & & & & 12 & 11 & & \\
\hline$m p-2$ & A. & $\begin{array}{l}0.0002 \\
0.029 \\
\end{array}$ & $\begin{array}{c}59 \\
6 \\
\ldots\end{array}$ & & & & & & & & $\begin{array}{l}7 \\
16 \\
\cdots\end{array}$ & & 28 & & & 17 & 13 & & \\
\hline WOLF CREEK & A & $\begin{array}{l}0.18 \\
0.011\end{array}$ & $\begin{array}{l}6 \\
80 \\
-\end{array}$ & & & & & & & & & & & & & 44 & 43 & 16 & 3 \\
\hline YANKEE ROWE & A. & $\begin{array}{r}0.45 \\
0.038 \\
0.038\end{array}$ & 11 & $\begin{array}{l}18 \\
47 \\
.\end{array}$ & & & & & & & & & 5 & & & 46 & 32 & & 21 \\
\hline Zion & $\therefore$ & $\begin{array}{r}6.8 \\
0.14 \\
-1.1\end{array}$ & $\begin{array}{r}56 \\
74 \\
-1 .\end{array}$ & & & & & & & & & & & & & 22 & 18 & 21 & 5 \\
\hline
\end{tabular}

- Contributions less than $3 x$ are not included in table. 
SITE SUMMARIES

1988

2.1 
Site: ARKANSAS ONE

POPE COUNTY, ARKANSAS

Location: $\quad$ N $35.3100^{\circ} \quad$ W $93.2308^{\circ}$

POPULATION DATA

Total Population Within 2-to-80-km Region: $1.9 E 5$

Major Metropolitan Centers Within Region:

\begin{tabular}{lccl}
\multicolumn{1}{c}{ Center } & Population & \multicolumn{2}{c}{ Location } \\
Russellville & 14,000 & $10 \mathrm{~km}$ & $\mathrm{E}$ \\
Conway & 20,000 & $76 \mathrm{~km}$ & ESE
\end{tabular}

SITE-SPECIFIC DATA - AIRBORNE PATHWAYS

Average Annual State Production

of Crops and Animal Products

In $80-\mathrm{km}$ Radius Circle

Veg: $5.8 \mathrm{E} 6 \mathrm{ki}$ logram

Milk: $4.8 E 7$ liter

Meat: $7.2 E 7$ kilogram

Regional Productivity Factor:

Animal Grazing Factor:

1

0.7

Meteorology Period of Record: 1 JAN 75 - 31 DEC 75 Recovery: $97 \%$

Drinking Water:

Fish:

SITE-SPECIFIC DATA - WATERBORNE PATHWAYS via ARKANSAS RIVER

Average River Flow

at Site: $36,000 \mathrm{ft}^{3} / \mathrm{s}$

None

Edible Harvest: $1.4^{(\mathrm{a})} \mathrm{kg} / \mathrm{yr}$

Dilution Factor: 1

(a) Average individual consumption rates as given in the Final Environmental statement (FES) (1973) were used in lieu of catch data. The reference to environmental statements here and on succeeding pages may be found in Table 3 . 


\section{POPULATION DOSE-COMMITMENT ESTIMATES AND \\ AVERAGE INDIVIDUAL DOSE-COMMITMENT HISTOGRAM FOR}

ARKANSAS ONE 1 AND 2

Dose Commitments (person-rem) from Waterborne Pathways

Total Body GI-LLI Ihyroid Bone Liver

$\begin{array}{llllll}\text { Infant } & 0.0 \mathrm{E}+00 & 0.0 \mathrm{E}+00 & 0.0 \mathrm{E}+00 & 0.0 \mathrm{E}+00 & 0.0 \mathrm{E}+00 \\ \text { Child } & 4.4 \mathrm{E}-02 & 1.8 \mathrm{E}-02 & 8.0 \mathrm{E}-03 & 2.4 \mathrm{E}-01 & 2.6 \mathrm{E}-01 \\ \text { Teen } & 8.4 \mathrm{E}-02 & 3.8 \mathrm{E}-02 & 5.8 \mathrm{E}-03 & 1.4 \mathrm{E}-01 & 2.2 \mathrm{E}-01 \\ \text { Adult } & 9.2 \mathrm{E}-01 & 3.3 \mathrm{E}-01 & 3.8 \mathrm{E}-02 & 8.3 \mathrm{E}-01 & 1.3 \mathrm{E}+00 \\ \text { TOTAL } & 1.0 \mathrm{E}+00 & 3.9 \mathrm{E}-01 & 5.2 \mathrm{E}-02 & 1.2 \mathrm{E}+00 & 1.8 \mathrm{E}+00\end{array}$

Dose Commitments (person-rem) from Airborne Pathways

Total Body GI-LLI Thyroid Bone Liver

$\begin{array}{lllllll}\text { Infant } & 4.9 \mathrm{E}-04 & 4.9 \mathrm{E}-04 & 6.7 \mathrm{E}-04 & 4.8 \mathrm{E}-04 & 4.9 \mathrm{E}-04 & 5.2 \mathrm{E}-04 \\ \text { Child } & 5.5 \mathrm{E}-03 & 5.4 \mathrm{E}-03 & 6.5 \mathrm{E}-03 & 5.3 \mathrm{E}-03 & 5.5 \mathrm{E}-03 & 5.9 \mathrm{E}-03 \\ \text { Teen } & 4.0 \mathrm{E}-03 & 4.0 \mathrm{E}-03 & 4.4 \mathrm{E}-03 & 3.9 \mathrm{E}-03 & 4.0 \mathrm{E}-03 & 4.6 \mathrm{E}-03 \\ \text { Adult } & 2.4 \mathrm{E}-02 & 2.4 \mathrm{E}-02 & 2.6 \mathrm{E}-02 & 2.4 \mathrm{E}-02 & 2.4 \mathrm{E}-02 & 2.6 \mathrm{E}-02 \\ \text { TOTAL } & 3.4 \mathrm{E}-02 & 3.4 \mathrm{E}-02 & 3.7 \mathrm{E}-02 & 3.3 \mathrm{E}-02 & 3.4 \mathrm{E}-02 & 3.7 \mathrm{E}-02\end{array}$

Production/Consumption factors: (a)

Produce: 0.15 Milk: $1.9 \quad$ Meat: 4.6

Fraction of Population Receiving an Average Individual

Total-Body Dose Commitment from Alrborne Pathways

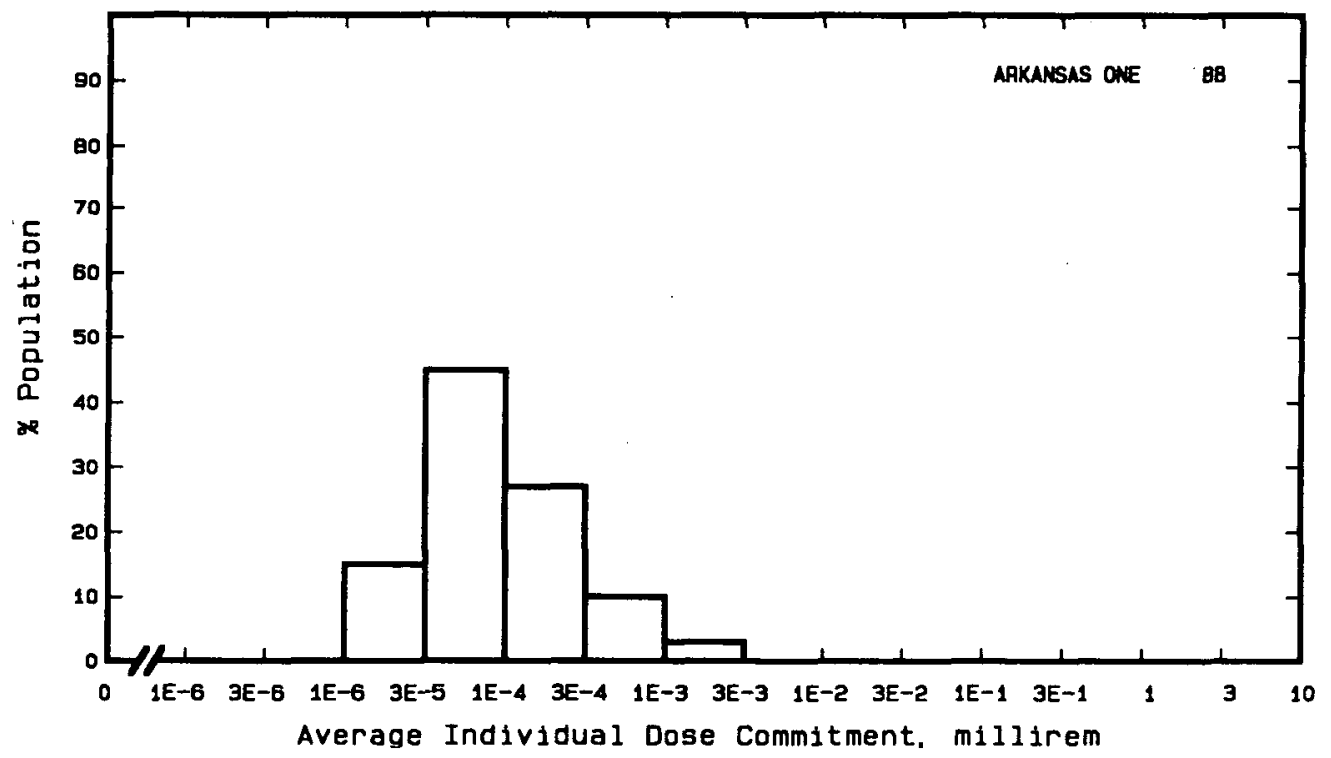

(a) See Appendix A, Page A-5, for explanation of this ratio. 
Site: BEAVER VALLEY

Location: $\quad N 40.6219^{\circ} \quad$ W $80.4339^{\circ}$

POPULATION DATA

Total Population Within 2-to-80-km Region: 3.7E6

Major Metropolitan Centers Within Region:

Center

Pittsburgh SMSA

Youngstown-Warren SMSA

Stuebenville-Weirton SMSA

Wheel ing SMSA

New Castle
Population

$2,100,000$

520,000

160,000

190,000

35,000
Location

$42 \mathrm{~km} \quad \mathrm{ESE}$

$56 \mathrm{~km} \quad \mathrm{NNW}$

$33 \mathrm{~km} \quad \mathrm{SSW}$

$66 \mathrm{~km} \quad \mathrm{SSW}$

$43 \mathrm{~km} \mathrm{~N}$

SITE-SPECIFIC DATA - AIRBORNE PATHWAYS

Average Annual State Production

of Crops and Animal Products

In $80-\mathrm{km}$ Radius Circle

Regional Productivity Factor:

Animal Grazing Factor:
Veg: $5.3 E 7$ kilogram

Milk: 5.3E8 liter

Meat: 5.4E7 kilogram

1

0.5

Meteorology Period of Record: 1 JAN 77 - 31 DEC 77 Recovery: 92\%

SITE-SPECIFIC DATA - WATERBORNE PATHWAYS via OHIO RIVER

Average River Flow

at Site: $30,000 \mathrm{ft}^{3} / \mathrm{s}$

Drinking Water:

Exposed Population: 6,200

Fish:

Edible Harvest: $410 \mathrm{~kg} / \mathrm{yr}$

Dilution Factor: 1

(a) This factor accounts for the incomplete dilution of plant effluent by river at point of drinking water intake at Midland. 


\section{POPULATION DOSE-COMMITMENT ESTIMATES AND \\ AVERAGE INDIVIDUAL DOSE-COMMITMENT HISTOGRAM FOR}

BEAVER VALLEY 1 AND 2

Dose Commitments (person-rem) from Waterborne Pathways

Total Body GI-LLI Ihyroid Bone Liver

$\begin{array}{llllll}\text { Infant } & 8.7 \mathrm{E}-04 & 8.7 \mathrm{E}-04 & 9.0 \mathrm{E}-04 & 1.4 \mathrm{E}-05 & 8.9 \mathrm{E}-04 \\ \text { Child } & 9.8 \mathrm{E}-03 & 9.9 \mathrm{E}-03 & 9.9 \mathrm{E}-03 & 1.6 \mathrm{E}-04 & 9.9 \mathrm{E}-03 \\ \text { Teen } & 3.7 \mathrm{E}-03 & 3.9 \mathrm{E}-03 & 3.8 \mathrm{E}-03 & 4.0 \mathrm{E}-05 & 3.8 \mathrm{E}-03 \\ \text { Adult } & 3.2 \mathrm{E}-02 & 3.3 \mathrm{E}-02 & 3.2 \mathrm{E}-02 & 2.5 \mathrm{E}-04 & 3.2 \mathrm{E}-02 \\ \text { TOTAL } & 4.6 \mathrm{E}-02 & 4.8 \mathrm{E}-02 & 4.7 \mathrm{E}-02 & 4.6 \mathrm{E}-04 & 4.7 \mathrm{E}-02\end{array}$

Dose Commitments (person-rem) from Airborne Pathways

Total Body GI-LLI Thyroid Bone Liver

$\begin{array}{lllllll}\text { Infant } & 1.5 \mathrm{E}-03 & 1.4 \mathrm{E}-03 & 5.4 \mathrm{E}-03 & 1.3 \mathrm{E}-04 & 1.5 \mathrm{E}-03 & 1.5 \mathrm{E}-03 \\ \text { Child } & 1.7 \mathrm{E}-02 & 1.7 \mathrm{E}-02 & 3.9 \mathrm{E}-02 & 1.3 \mathrm{E}-03 & 1.7 \mathrm{E}-02 & 1.8 \mathrm{E}-02 \\ \text { Teen } & 1.1 \mathrm{E}-02 & 1.1 \mathrm{E}-02 & 2.0 \mathrm{E}-02 & 8.7 \mathrm{E}-04 & 1.1 \mathrm{E}-02 & 1.2 \mathrm{E}-02 \\ \text { Adult } & 5.6 \mathrm{E}-02 & 5.6 \mathrm{E}-02 & 8.8 \mathrm{E}-02 & 5.0 \mathrm{E}-03 & 5.6 \mathrm{E}-02 & 6.0 \mathrm{E}-02 \\ \text { TOTAL } & 8.5 \mathrm{E}-02 & 8.5 \mathrm{E}-02 & 1.5 \mathrm{E}-01 & 7.3 \mathrm{E}-03 & 8.6 \mathrm{E}-02 & 9.1 \mathrm{E}-02\end{array}$

Production/Consumption factors:

Produce: 0.075 Milk: $1.1 \quad$ Meat: 0.19

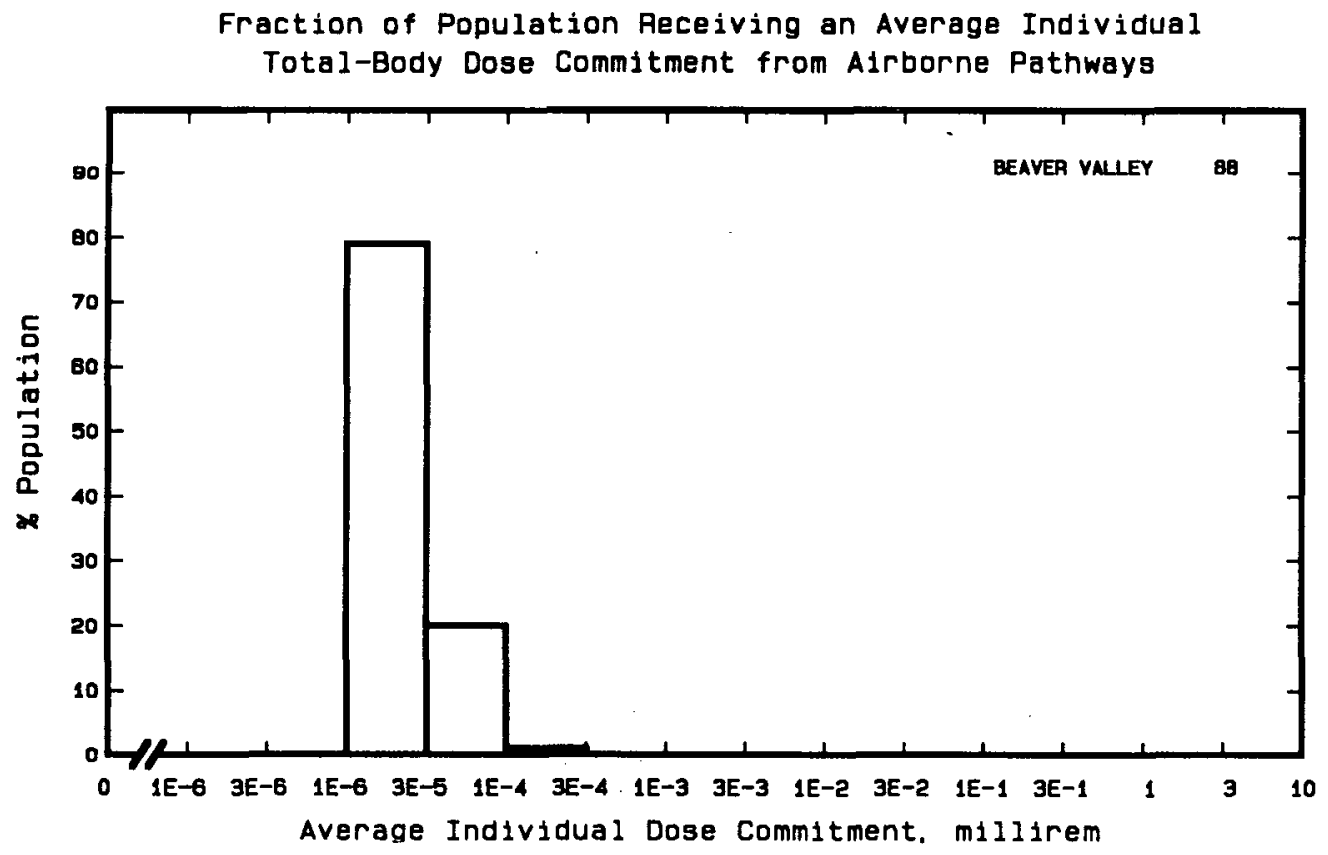


Site: BIG ROCK POINT

CHARLEVOIX COUNTY, MICHIGAN

Location: $\quad$ N $45.3592^{\circ} \quad$ W $85.1947^{\circ}$

POPULATION DATA

Total Population Within 2-to-80-km Region: $1.7 E 5$

Major Metropolitan Centers Within Region:

Center

Traverse City

Petoskey

Cheboygan
Population

16,000

6,100

5,100
Location

$75 \mathrm{~km} \quad \mathrm{SSW}$

$18 \mathrm{~km} \quad \mathrm{E}$

$65 \mathrm{~km}$ ENE

SITE-SPECIFIC DATA - AIRBORNE PATHWAYS

Average Annual State Production

of Crops and Animal Products

In $80-\mathrm{km}$ Radius Circle

Regiona1 Productivity Factor:

Animal Grazing Factor:
Veg: $6.9 \mathrm{E7}$ kilogram

Milk: 2.9E8 liter

Meat: $4.5 \mathrm{E} 7$ kilogram

0.5

0.5

Meteorology Period of Record: 9 FEB 61 - 8 FEB 63 Recovery: 85\%

Average Dilution Flow

from Plant: $92 \mathrm{ft}^{3} / \mathrm{s}$

Drinking Water:

Exposed Population: 31,000 (a)

Dilution Factor: 4.9E-5(b)

Fish:

Edible Harvest: (c) $\mathrm{kg} / \mathrm{yr}$

Dilution Factor: 0.01

(a) Population exposed to contaminated drinking water derived from information obtained from J. Hennigan, Division of Radiation Health, Bureau of Environment and Heaith. Michigan Department of Public Health.

(b) Drinking water dilution factor estimated by averaging dilution factor derived from Figure 6B-5, Vol. 1 of WASH-1258 (1973) suitably weighted for population.

(c) Generic consumption rate used (Table A-1). 


\section{POPULATION DOSE-COMMITMENT ESTIMATES AND \\ AVERAGE INDIVIDUAL DOSE-COMMITMENT HISTOGRAM FOR}

BIG ROCK POINT

Dose Commitments (person-rem) from Waterborne Pathways

Total Body GI-LLI Ihyroid Bone Liver

$\begin{array}{llllll}\text { Infant } & 9.8 \mathrm{E}-07 & 1.5 \mathrm{E}-07 & 1.7 \mathrm{E}-07 & 4.2 \mathrm{E}-06 & 1.2 \mathrm{E}-06 \\ \text { Child } & 3.4 \mathrm{E}-02 & 3.4 \mathrm{E}-03 & 7.5 \mathrm{E}-05 & 2.0 \mathrm{E}-01 & 1.9 \mathrm{E}-01 \\ \text { Teen } & 6.1 \mathrm{E}-02 & 7.3 \mathrm{E}-03 & 5.4 \mathrm{E}-05 & 1.2 \mathrm{E}-01 & 1.6 \mathrm{E}-01 \\ \text { Adult } & 6.5 \mathrm{E}-01 & 6.3 \mathrm{E}-02 & 3.5 \mathrm{E}-04 & 7.3 \mathrm{E}-01 & 9.4 \mathrm{E}-01 \\ \text { TOTAL } & 7.5 \mathrm{E}-01 & 7.4 \mathrm{E}-02 & 4.8 \mathrm{E}-04 & 1.1 \mathrm{E}+00 & 1.3 \mathrm{E}+00\end{array}$

Dose Commitments (person-rem) from Airborne Pathways

Total Body GI-LLI Thyroid Bone Liver Lung

$\begin{array}{lllllll}\text { Infant } & 1.4 \mathrm{E}-03 & 1.4 \mathrm{E}-03 & 1.6 \mathrm{E}-03 & 1.4 \mathrm{E}-03 & 1.4 \mathrm{E}-03 & 1.5 \mathrm{E}-03 \\ \text { Child } & 1.6 \mathrm{E}-02 & 1.6 \mathrm{E}-02 & 1.8 \mathrm{E}-02 & 1.6 \mathrm{E}-02 & 1.6 \mathrm{E}-02 & 1.6 \mathrm{E}-02 \\ \text { Teen } & 1.2 \mathrm{E}-02 & 1.2 \mathrm{E}-02 & 1.2 \mathrm{E}-02 & 1.2 \mathrm{E}-02 & 1.2 \mathrm{E}-02 & 1.2 \mathrm{E}-02 \\ \text { Adult } & 7.1 \mathrm{E}-02 & 7.1 \mathrm{E}-02 & 7.4 \mathrm{E}-02 & 7.0 \mathrm{E}-02 & 7.1 \mathrm{E}-02 & 7.3 \mathrm{E}-02 \\ \text { TOTAL } & 1.0 \mathrm{E}-01 & 1.0 \mathrm{E}-01 & 1.1 \mathrm{E}-01 & 9.9 \mathrm{E}-02 & 1.0 \mathrm{E}-01 & 1.0 \mathrm{E}-01\end{array}$

Production/Consumption factors:

Produce: 1.1

Mi1k: 6.5

Meat: 1.7

Fraction of Population Receiving an Average Individual Total-Body Dose Commitment from Airborne Pathways

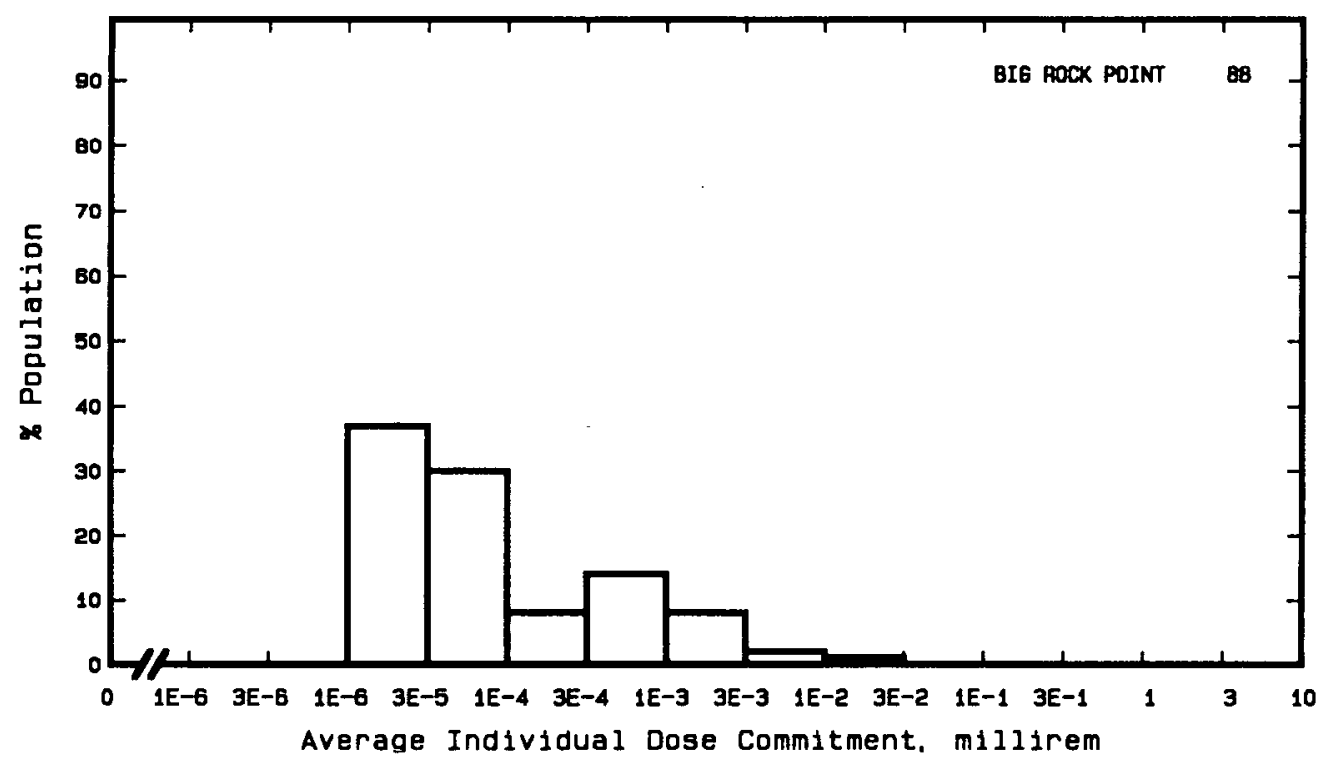


Site: BRAIDWOOD

BRAIDWOOD, ILLINOIS

Location: $\quad$ N $41.2683^{\circ}$ W $88.2133^{\circ}$

POPULATION DATA

Total Population Within 2-to-80-km Region: 4.3E6

Major Metropolitan Centers Within Region:

Center

Chicago SMSA

Gary-Hammond-E.Chicago-SMSA

Kankakee SMSA

Aurora

Jol iet
Population

$7,100,000(a)$
$640,000(a)$
100,000
81,000
78,000

\section{Location}

$70 \mathrm{~km} \quad \mathrm{NE}$

$70 \mathrm{~km} \quad \mathrm{ENE}$

$32 \mathrm{~km} \quad \mathrm{ESE}$

$55 \mathrm{~km} \quad \mathrm{~N}$

$40 \mathrm{~km} \quad \mathrm{NNE}$

\section{SITE-SPECIFIC DATA - AIRBORNE PATHWAYS}

Average Annual State Production

of Crops and Animal Products

In $80-\mathrm{km}$ Radius Circle

Regional Productivity Factor:

Animal Grazing Factor:
Veg: 1.1 E8 kilogram

Milk: 1.8E8 liter

Meat: $1.9 \mathrm{E} 8 \mathrm{ki}$ logram

0.9

0.5

Meteorology Period of Record: 1 JAN 79 - 31 DEC 82 Recovery: 98\%

SITE-SPECIFIC DATA - WATERBORNE PATHWAYS via KANKAKEE RIVER

Average River $\mathrm{Flow}$

at Site: $3,600 \mathrm{ft}^{3} / \mathrm{s}$

Drinking Water:

Fish:
None

Edible Harvest: $0.18^{\text {(b) }} \mathrm{kg} / \mathrm{yr}$ Dilution Factor: 1

(a) Population of total SMSA given; population of SMSA fraction within $80 \mathrm{~km}$ of site would be somewhat smaller.

(b) Ten percent of population are assumed to obtain fish from river. Average individual consumption rate of $5 \mathrm{~g} / \mathrm{d}$ given in FES (1974) used in 1 ieu of catch data. 


\section{POPULATION DOSE-COMMITMENT ESTIMATES AND \\ AVERAGE INDIVIDUAL DOSE-COMMITMENT HISTOGRAM FOR}

BRAIDWOOD 1 and 2

Dose Commitments (person-rem) from Waterborne Pathways

Total Body GI-LLI $\underline{\text { Thyroid }}$ Bone Liver

$\begin{array}{llllll}\text { Infant } & 0.0 \mathrm{E}+00 & 0.0 \mathrm{E}+00 & 0.0 \mathrm{E}+00 & 0.0 \mathrm{E}+00 & 0.0 \mathrm{E}+00 \\ \text { Child } & 8.3 \mathrm{E}-02 & 1.2 \mathrm{E}-01 & 2.6 \mathrm{E}-02 & 1.9 \mathrm{E}-01 & 2.4 \mathrm{E}-01 \\ \text { Teen } & 9.9 \mathrm{E}-02 & 2.7 \mathrm{E}-01 & 1.9 \mathrm{E}-02 & 1.2 \mathrm{E}-01 & 2.1 \mathrm{E}-01 \\ \text { Adult } & 9.1 \mathrm{E}-01 & 2.4 \mathrm{E}+00 & 1.3 \mathrm{E}-01 & 6.7 \mathrm{E}-01 & 1.2 \mathrm{E}+00 \\ \text { TOTAL } & 1.1 \mathrm{E}+00 & 2.8 \mathrm{E}+00 & 1.7 \mathrm{E}-01 & 9.8 \mathrm{E}-01 & 1.7 \mathrm{E}+00\end{array}$

Dose Commitments (person-rem) from Airborne Pathways

Total Body GI-LLI Thyroid Bone Liver Lung

$\begin{array}{lllllll}\text { Infant } & 7.3 \mathrm{E}-05 & 7.3 \mathrm{E}-05 & 3.7 \mathrm{E}-04 & 4.5 \mathrm{E}-05 & 7.4 \mathrm{E}-05 & 7.6 \mathrm{E}-05 \\ \text { Child } & 1.0 \mathrm{E}-03 & 1.0 \mathrm{E}-03 & 3.3 \mathrm{E}-03 & 4.9 \mathrm{E}-04 & 1.0 \mathrm{E}-03 & 1.1 \mathrm{E}-03 \\ \text { Teen } & 7.2 \mathrm{E}-04 & 7.2 \mathrm{E}-04 & 1.6 \mathrm{E}-03 & 3.6 \mathrm{E}-04 & 7.2 \mathrm{E}-04 & 7.9 \mathrm{E}-04 \\ \text { Adult } & 4.3 \mathrm{E}-03 & 4.3 \mathrm{E}-03 & 7.7 \mathrm{E}-03 & 2.2 \mathrm{E}-03 & 4.3 \mathrm{E}-03 & 4.5 \mathrm{E}-03 \\ \text { TOTAL } & 6.1 \mathrm{E}-03 & 6.1 \mathrm{E}-03 & 1.3 \mathrm{E}-02 & 3.1 \mathrm{E}-03 & 6.1 \mathrm{E}-03 & 6.4 \mathrm{E}-03\end{array}$

Production/Consumption factors:

Produce: $0.12 \quad$ Milk: $0.28 \quad$ Meat: 0.49

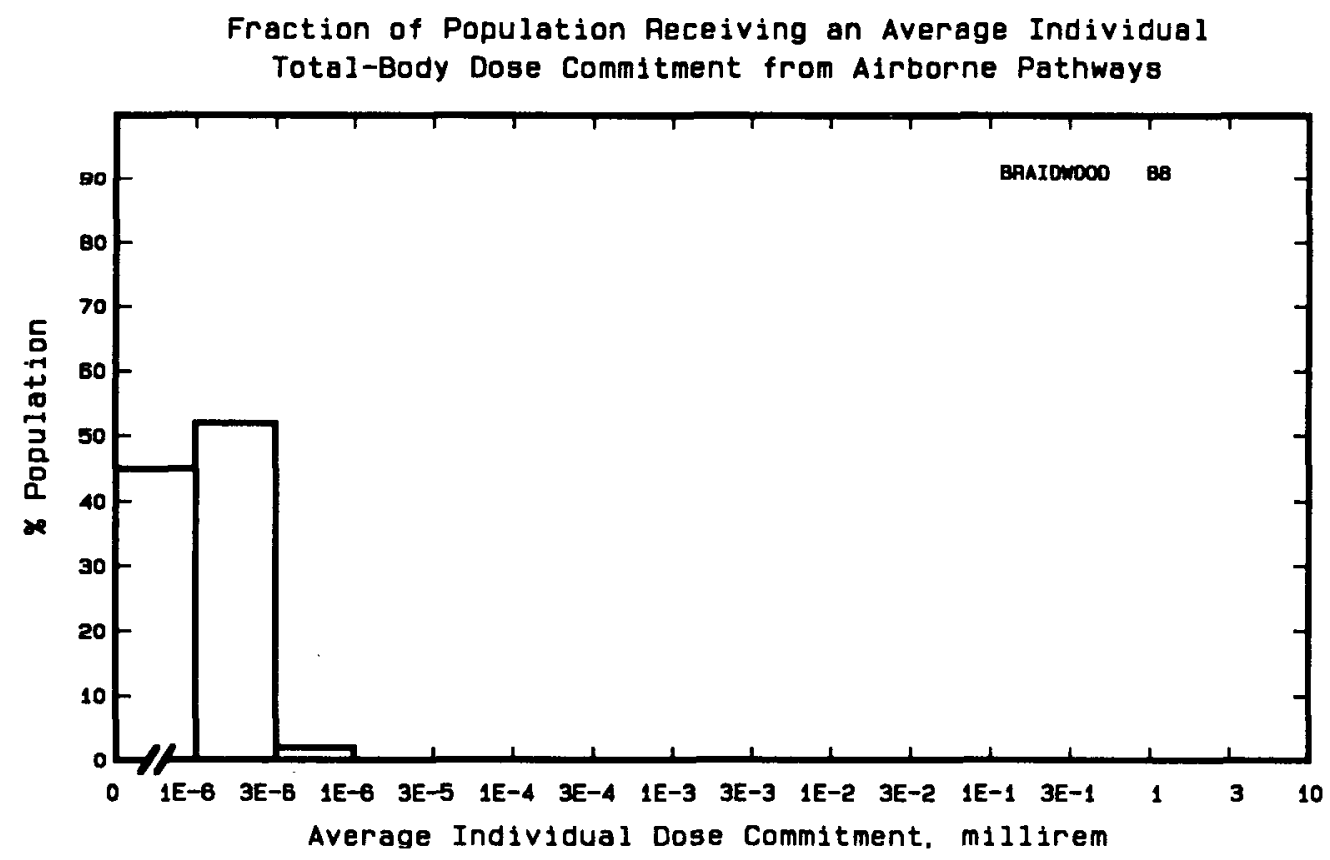


Site: BROWNS FERRY

Location: $\quad$ N $34.7042^{\circ}$ W $87.1186^{\circ}$

POPULATION DATA

Total Population Within 2-to-80-km Region: 7.5E5

Major Metropolitan Centers Within Region:

Center

Huntsville SMSA

Florence SMSA

Decatur

Athens

Cullman
Population

310,000

140,000

43,000

15,000

13,000
Location

$49 \mathrm{~km} \quad \mathrm{E}$

$52 \mathrm{~km}$ WNW

$16 \mathrm{~km} \quad \mathrm{SE}$

$17 \mathrm{~km} \quad \mathrm{NE}$

$64 \mathrm{~km}$ SSE

SITE-SPECIFIC DATA - AIRBORNE PATHWAYS

Average Annual State Production

of Crops and Animal Products

In $80-\mathrm{km}$ Radius Circle

Regional Productivity Factor:

Animal Grazing Factor:
Veg: $1.7 E 7$ kilogram

Milk: 5.7E7 liter

Meat: $8.6 \mathrm{E} 7 \mathrm{kilogram}$

1

0.7

Meteorology Period of Record: 1 JAN 74 - 31 DEC 75 Recovery: $94 \%$

Orinking Water:

Fish:
Average River Flow at Site: $45,000 \mathrm{ft}^{3} / \mathrm{s}$

Exposed Population: 26,000 Dilution Factor: 1

Edible Harvest: $1.6 \mathrm{E} 6 \mathrm{~kg} / \mathrm{yr}$ Dilution Factor: 1 


\section{POPULATION DOSE-COMMITMENT ESTIMATES AND \\ AVERAGE INDIVIDUAL DOSE-COMMITMENT HISTOGRAM FOR \\ BROWNS FERRY 1, 2, AND 3}

Dose Commitments (person-rem) from Waterborne Pathways

Total Body GI-LLI Thyroid Bone Liver

$\begin{array}{llllll}\text { Infant } & 1.8 \mathrm{E}-05 & 3.8 \mathrm{E}-06 & 7.2 \mathrm{E}-07 & 1.5 \mathrm{E}-04 & 1.9 \mathrm{E}-04 \\ \text { Child } & 4.9 \mathrm{E}-02 & 2.2 \mathrm{E}-03 & 8.7 \mathrm{E}-06 & 2.8 \mathrm{E}-01 & 3.0 \mathrm{E}-01 \\ \text { Teen } & 9.3 \mathrm{E}-02 & 4.3 \mathrm{E}-03 & 3.7 \mathrm{E}-06 & 1.7 \mathrm{E}-01 & 2.5 \mathrm{E}-01 \\ \text { Adult } & 1.0 \mathrm{E}+00 & 3.5 \mathrm{E}-02 & 3.1 \mathrm{E}-05 & 9.6 \mathrm{E}-01 & 1.5 \mathrm{E}+00 \\ \text { TOTAL } & 1.2 \mathrm{E}+00 & 4.2 \mathrm{E}-02 & 4.4 \mathrm{E}-05 & 1.4 \mathrm{E}+00 & 2.0 \mathrm{E}+00\end{array}$

Dose Commitments (person-rem) from Airborne Pathways

Total Body GI-LLI Thyroid Bone Liver $\underline{\text { Lung }}$

$\begin{array}{lllllll}\text { Infant } & 1.5 \mathrm{E}-05 & 1.4 \mathrm{E}-05 & 1.4 \mathrm{E}-05 & 1.9 \mathrm{E}-05 & 2.2 \mathrm{E}-05 & 2.9 \mathrm{E}-05 \\ \text { Child } & 1.8 \mathrm{E}-04 & 1.9 \mathrm{E}-04 & 1.6 \mathrm{E}-04 & 2.0 \mathrm{E}-04 & 2.3 \mathrm{E}-04 & 4.1 \mathrm{E}-04 \\ \text { Teen } & 1.3 \mathrm{E}-04 & 1.5 \mathrm{E}-04 & 1.2 \mathrm{E}-04 & 1.2 \mathrm{E}-04 & 1.5 \mathrm{E}-04 & 3.4 \mathrm{E}-04 \\ \text { Adult } & 8.0 \mathrm{E}-04 & 9.8 \mathrm{E}-04 & 7.0 \mathrm{E}-04 & 6.5 \mathrm{E}-04 & 8.1 \mathrm{E}-04 & 1.6 \mathrm{E}-03 \\ \text { TOTAL } & 1.1 \mathrm{E}-03 & 1.3 \mathrm{E}-03 & 9.9 \mathrm{E}-04 & 9.8 \mathrm{E}-04 & 1.2 \mathrm{E}-03 & 2.4 \mathrm{E}-03\end{array}$

Production/Consumption factors:

Produce: 0.12 Milk: $0.58 \quad$ Meat: 1.4

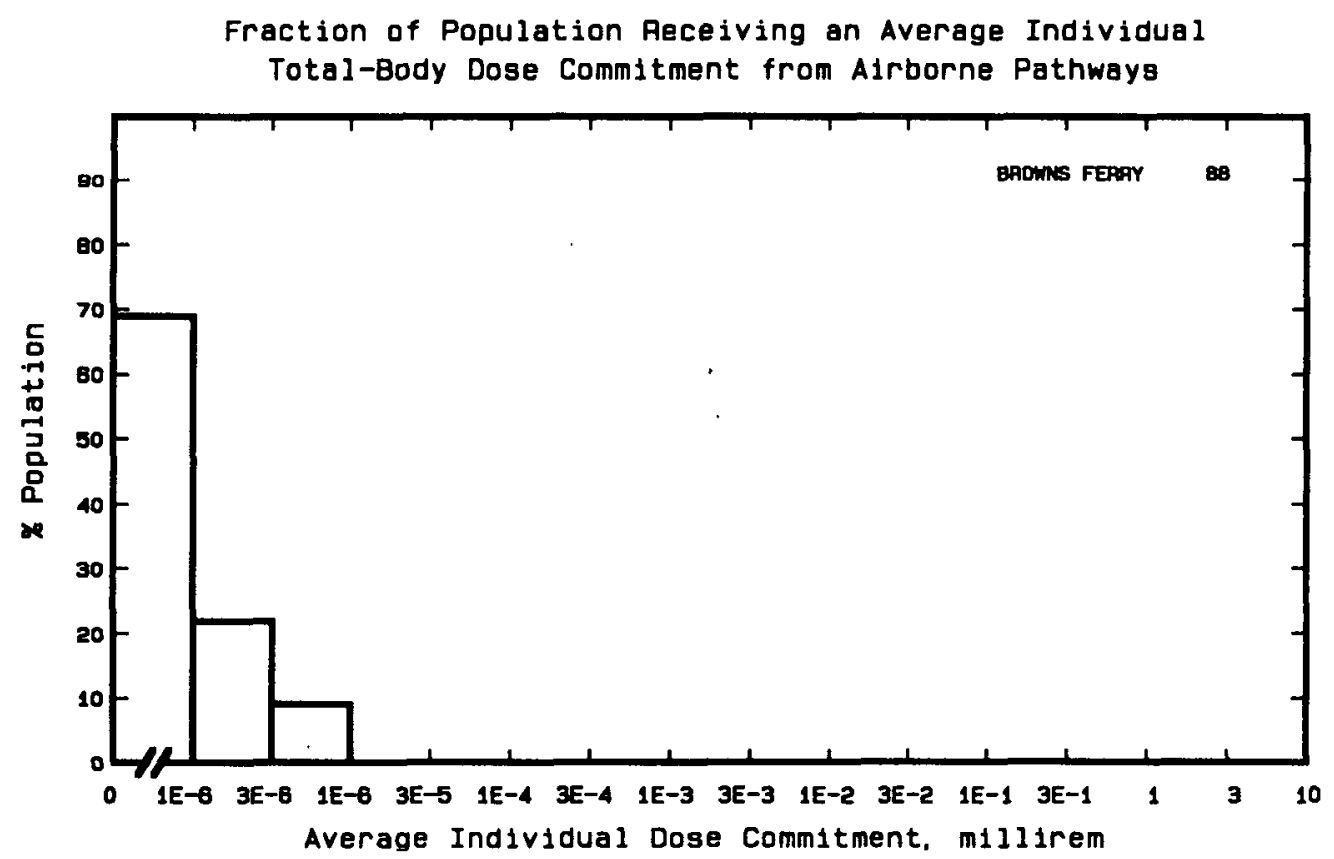


Site: BRUNSWICK

Location: $\quad$ N $33.9583^{\circ} \quad$ W $78.0106^{\circ}$
BRUNSWICK COUNTY, NORTH CAROLINA

POPULATION DATA

Total Population Within 2-to-80-km Region: 2.4E5

- Major Metropolitan Centers Within Region:

Center Population Location

$\begin{array}{lrrr}\text { Wilmington SMSA } & 140,000 & 32 \mathrm{~km} & \text { NNE } \\ \text { Whiteville } & 5,800 & 75 \mathrm{~km} & \text { WNW } \\ \text { N. Myrtle Beach } & 4,200 & 65 \mathrm{~km} & \text { WSW }\end{array}$

SITE-SPECIFIC DATA - AIRBORNE PATHWAYS

Average Annual State Production

of Crops and Animal Products

In $80-\mathrm{km}$ Radius Circle

Regional Productivity Factor:

Animal Grazing Factor:
Veg: $2.6 \mathrm{E} 7 \mathrm{ki}$ logram

Milk: $1.0 \mathrm{E} 8$ liter

Meat: $5.8 \mathrm{E} 7$ kilogram

0.3

0.7

Meteorology Period of Record: 1 JAN 75 - 31 DEC 75 Recovery: 93\%

SITE-SPECIFIC DATA - WATERBORNE PATHWAYS via ATLANTIC OCEAN

Average Dilution Flow

from Plant: $200 \mathrm{ft}^{3} / \mathrm{s}$

Fish:

Edible Harvest: $2.1 \mathrm{E5}(\mathrm{a}) \mathrm{kg} / \mathrm{yr}$ Dilution Factor: 0.001

Invertebrates:

Edible Harvest: $1.1 \mathrm{E} 5(\mathrm{a}) \mathrm{kg} / \mathrm{yr}$ Dilution Factor: 0.002

(a) Fish and invertebrate harvests together total harvest given in FES (1973). 


\section{POPULATION DOSE-COMMITMENT ESTIMATES AND \\ AVERAGE INDIVIDUAL DOSE-COMMITMENT HISTOGRAM FOR \\ BRUNSWICK 1 AND 2}

Dose Commitments (person-rem) from Waterborne Pathways

Total Body GI-LLI thyroid Bone Liver

$\begin{array}{llllll}\text { Infant } & 0.0 \mathrm{E}+00 & 0.0 \mathrm{E}+00 & 0.0 \mathrm{E}+00 & 0.0 \mathrm{E}+00 & 0.0 \mathrm{E}+00 \\ \text { Child } & 7.1 \mathrm{E}-04 & 1.2 \mathrm{E}-03 & 1.8 \mathrm{E}-04 & 1.1 \mathrm{E}-03 & 1.2 \mathrm{E}-03 \\ \text { Teen } & 5.1 \mathrm{E}-04 & 2.5 \mathrm{E}-03 & 1.3 \mathrm{E}-04 & 6.5 \mathrm{E}-04 & 1.0 \mathrm{E}-03 \\ \text { Adult } & 3.4 \mathrm{E}-03 & 2.2 \mathrm{E}-02 & 8.5 \mathrm{E}-04 & 3.8 \mathrm{E}-03 & 6.1 \mathrm{E}-03 \\ \text { TOTAL } & 4.7 \mathrm{E}-03 & 2.5 \mathrm{E}-02 & 1.2 \mathrm{E}-03 & 5.6 \mathrm{E}-03 & 8.4 \mathrm{E}-03\end{array}$

Dose Commitments (person-rem) from Airborne Pathways

Total Body GI-LLI Thyroid Bone Liver Lung

$\begin{array}{lllllll}\text { Infant } & 5.1 \mathrm{E}-04 & 5.1 \mathrm{E}-04 & 4.1 \mathrm{E}-03 & 5.2 \mathrm{E}-04 & 5.3 \mathrm{E}-04 & 5.5 \mathrm{E}-04 \\ \text { Child } & 5.7 \mathrm{E}-03 & 5.7 \mathrm{E}-03 & 2.7 \mathrm{E}-02 & 5.8 \mathrm{E}-03 & 5.8 \mathrm{E}-03 & 6.4 \mathrm{E}-03 \\ \text { Teen } & 4.1 \mathrm{E}-03 & 4.2 \mathrm{E}-03 & 1.3 \mathrm{E}-02 & 4.1 \mathrm{E}-03 & 4.2 \mathrm{E}-03 & 4.8 \mathrm{E}-03 \\ \text { Adult } & 2.5 \mathrm{E}-02 & 2.6 \mathrm{E}-02 & 5.5 \mathrm{E}-02 & 2.5 \mathrm{E}-02 & 2.5 \mathrm{E}-02 & 2.8 \mathrm{E}-02 \\ \text { TOTAL } & 3.5 \mathrm{E}-02 & 3.6 \mathrm{E}-02 & 9.9 \mathrm{E}-02 & 3.5 \mathrm{E}-02 & 3.6 \mathrm{E}-02 & 3.9 \mathrm{E}-02\end{array}$

Production/Consumption factors:

Produce: $0.17 \quad$ Milk: $1.0 \quad$ Meat: 0.91
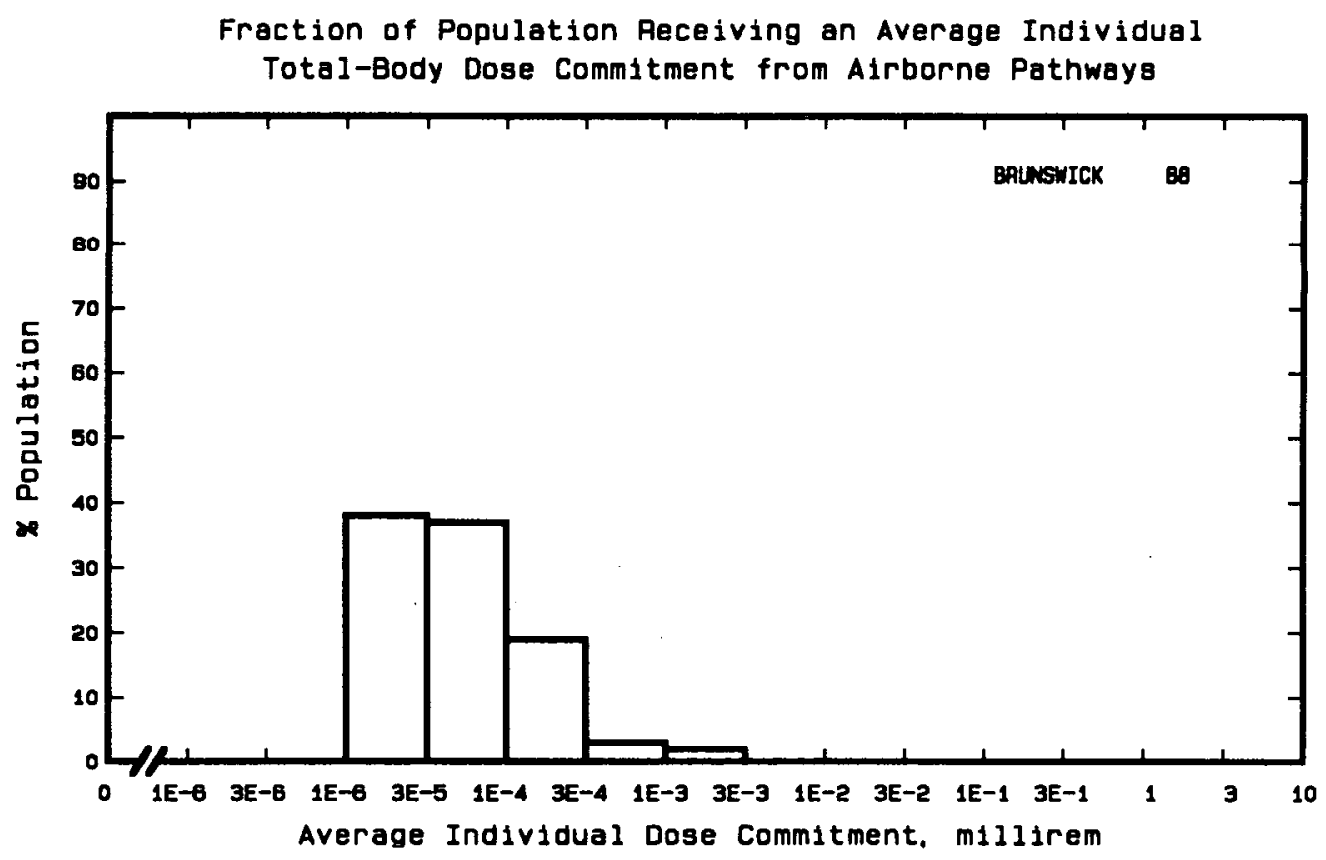
Site: BYRON

BYRON, ILLINOIS

Location: $\quad N \quad 42.1300^{\circ} \quad$ W $89.2550^{\circ}$

POPULATION DATA

Total Population Within 2-to-80-km Region: 9.4E5

Major Metropolitan Centers Within Region:

$\quad$ Center
Rockford SMSA
Freeport
Belvedere
Janesville
Beloit
De Kalb
Elgin

Population

280,000

26,000

42,000

61,000

35,000

33,000

64,000

\section{Location}

$27 \mathrm{~km} \quad \mathrm{NE}$

$35 \mathrm{~km} \quad \mathrm{NNW}$

$40 \mathrm{~km} \quad \mathrm{NE}$

$67 \mathrm{~km} \mathrm{~N}$

$48 \mathrm{~km} \quad \mathrm{NNE}$

$48 \mathrm{~km} \quad \mathrm{ESE}$

$80 \mathrm{~km} \quad \mathrm{E}$

SITE-SPECIFIC DATA - AIRBORNE PATHWAYS

Average Annual State Production

of Crops and Animal Products

In $80-\mathrm{km}$ Radius Circle

Regional Productivity Factor:

Animal Grazing Factor:
Veg: $1.1 \mathrm{E} 8 \mathrm{ki}$ logram

Milk: 1.8E8 liter

Meat: $1.9 \mathrm{E} 8 \mathrm{kilogram}$

0.5

Meteorology Period of Record: 1 JAN 74 - 31 DEC 74 Recovery: 95\%

Drinking Water: None

Fish:

Edible Harvest: $2.1 \mathrm{E} 4 \mathrm{~kg} / \mathrm{yr}$

Dilution Factor: 1 
Dose Commitments (person-rem) from Waterborne Pathways

Total Body GI-LLI Ihyroid $\underline{\text { Bone }}$ Liver

$\begin{array}{llllll}\text { Infant } & 0.0 \mathrm{E}+00 & 0.0 \mathrm{E}+00 & 0.0 \mathrm{E}+00 & 0.0 \mathrm{E}+00 & 0.0 \mathrm{E}+00 \\ \text { Child } & 4.0 \mathrm{E}-03 & 5.6 \mathrm{E}-03 & 4.5 \mathrm{E}-04 & 1.7 \mathrm{E}-02 & 2.1 \mathrm{E}-02 \\ \text { Teen } & 7.5 \mathrm{E}-03 & 1.2 \mathrm{E}-02 & 3.3 \mathrm{E}-04 & 1.0 \mathrm{E}-02 & 1.8 \mathrm{E}-02 \\ \text { Adult } & 8.0 \mathrm{E}-02 & 1.0 \mathrm{E}-01 & 2.3 \mathrm{E}-03 & 6.1 \mathrm{E}-02 & 1.1 \mathrm{E}-01 \\ \text { TOTAL } & 9.2 \mathrm{E}-02 & 1.2 \mathrm{E}-01 & 3.0 \mathrm{E}-03 & 8.9 \mathrm{E}-02 & 1.5 \mathrm{E}-01\end{array}$

Dose Commitments (person-rem) from Airborne Pathways

Total Body GI-LLI Ihyroid Bone Liver Lung

$\begin{array}{lllllll}\text { Infant } & 4.2 \mathrm{E}-04 & 4.1 \mathrm{E}-04 & 6.2 \mathrm{E}-03 & 4.2 \mathrm{E}-04 & 4.3 \mathrm{E}-04 & 4.4 \mathrm{E}-04 \\ \text { Child } & 4.6 \mathrm{E}-03 & 4.6 \mathrm{E}-03 & 5.0 \mathrm{E}-02 & 4.6 \mathrm{E}-03 & 4.7 \mathrm{E}-03 & 5.1 \mathrm{E}-03 \\ \text { Teen } & 3.4 \mathrm{E}-03 & 3.3 \mathrm{E}-03 & 2.1 \mathrm{E}-02 & 3.3 \mathrm{E}-03 & 3.4 \mathrm{E}-03 & 4.0 \mathrm{E}-03 \\ \text { Adult } & 2.0 \mathrm{E}-02 & 2.0 \mathrm{E}-02 & 8.5 \mathrm{E}-02 & 2.0 \mathrm{E}-02 & 2.0 \mathrm{E}-02 & 2.2 \mathrm{E}-02 \\ \text { TOTAL } & 2.9 \mathrm{E}-02 & 2.9 \mathrm{E}-02 & 1.6 \mathrm{E}-01 & 2.8 \mathrm{E}-02 & 2.9 \mathrm{E}-02 & 3.2 \mathrm{E}-02\end{array}$

Production/Consumption factors:

Produce: 0.55 Milk: $1.3 \quad$ Meat: 2.2

Fraction of Population Receiving an Average Individual

Tota]-Body Dose Commitment from Airborne Pathways

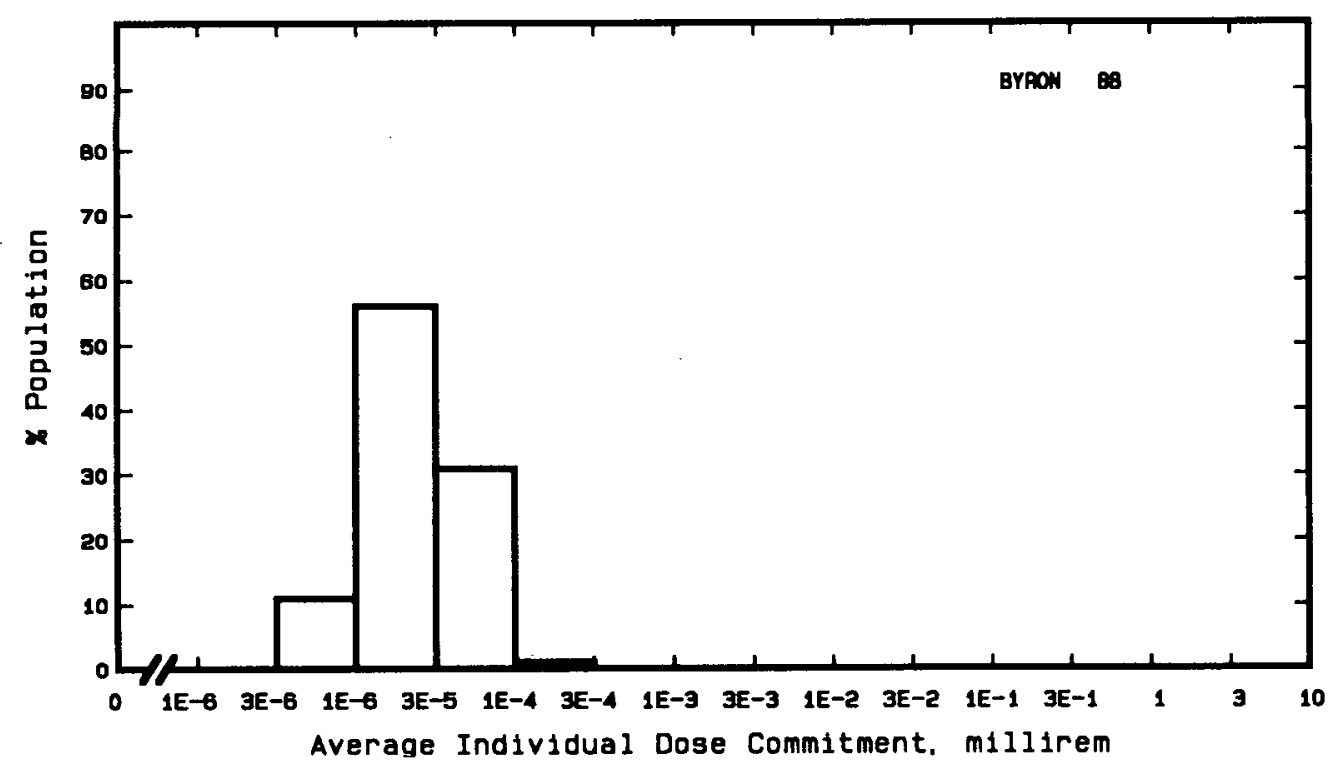


Site: CALLAWAY

FULTON, MISSOURI

Location: $\quad \mathrm{N} 38.7618^{\circ} \quad$ W $91.7979^{\circ}$

POPULATION DATA

Total Population Within 2-to-80-km Region: 3.7 E5

Major Metropolitan Centers Within Region:

Center

Columbia Jefferson City

Mexico

Washington

Fulton
Population

60,000

33,000

12,000

8,500

13,000

\section{Location}

$48 \mathrm{~km} \quad W N W$

$40 \mathrm{~km} \quad W S W$

$45 \mathrm{~km} \quad \mathrm{NNW}$

$69 \mathrm{~km}$ ESE

$19 \mathrm{~km} \quad \mathrm{NW}$

\section{SITE-SPECIFIC DATA - AIRBORNE PATHWAYS}

Average Annual State Production

of Crops and Animal Products

In $80-\mathrm{km}$ Radius Circle

Regional Productivity Factor:

Animal Grazing Factor:
Veg: $3.3 E 7$ kilogram

Mi1k: 1.5E8 liter

Meat: $1.9 \mathrm{E} 8$ kilogram

0.9

0.6

Meteorology Period of Record: 4 MAY 74 - 3 MAY 75 Recovery: $89 \%$

Average River Flow at Site: $80,000 \mathrm{ft}^{3} / \mathrm{s}$

Drinking Water:

Fish:
None

Edible Harvest: $1.0 \mathrm{E} 3 \mathrm{~kg} / \mathrm{yr}$ Dilution Factor: 1 


\section{POPULATION DOSE-COMMITMENT ESTIMATES AND AVERAGE INDIVIDUAL DOSE-COMMITMENT HISTOGRAM FOR \\ CALLAWAY}

Dose Commitments (person-rem) from Waterborne Pathways

Total Body GI-LLI $\underline{\text { Thyroid }}$ Bone Liver

$\begin{array}{llllll}\text { Infant } & 0.0 \mathrm{E}+00 & 0.0 \mathrm{E}+00 & 0.0 \mathrm{E}+00 & 0.0 \mathrm{E}+00 & 0.0 \mathrm{E}+00 \\ \text { Child } & 3.6 \mathrm{E}-06 & 4.9 \mathrm{E}-07 & 1.5 \mathrm{E}-07 & 1.7 \mathrm{E}-05 & 1.9 \mathrm{E}-05 \\ \text { Teen } & 6.7 \mathrm{E}-06 & 8.4 \mathrm{E}-07 & 1.3 \mathrm{E}-07 & 1.0 \mathrm{E}-05 & 1.6 \mathrm{E}-05 \\ \text { Adult } & 7.2 \mathrm{E}-05 & 7.0 \mathrm{E}-06 & 1.0 \mathrm{E}-06 & 6.1 \mathrm{E}-05 & 9.6 \mathrm{E}-05 \\ \text { TOTAL } & 8.2 \mathrm{E}-05 & 8.4 \mathrm{E}-06 & 1.3 \mathrm{E}-06 & 8.9 \mathrm{E}-05 & 1.3 \mathrm{E}-04\end{array}$

Dose Commitments (person-rem) from Airborne Pathways

Total Body GI-LLI Thyroid Bone Liver Lung

$\begin{array}{lllllll}\text { Infant } & 8.9 \mathrm{E}-05 & 8.9 \mathrm{E}-05 & 9.3 \mathrm{E}-05 & 6.5 \mathrm{E}-05 & 8.9 \mathrm{E}-05 & 9.5 \mathrm{E}-05 \\ \text { Child } & 1.1 \mathrm{E}-03 & 1.1 \mathrm{E}-03 & 1.2 \mathrm{E}-03 & 7.2 \mathrm{E}-04 & 1.1 \mathrm{E}-03 & 1.2 \mathrm{E}-03 \\ \text { Teen } & 7.7 \mathrm{E}-04 & 7.7 \mathrm{E}-04 & 7.9 \mathrm{E}-04 & 5.3 \mathrm{E}-04 & 7.7 \mathrm{E}-04 & 8.9 \mathrm{E}-04 \\ \text { Adult } & 4.5 \mathrm{E}-03 & 4.5 \mathrm{E}-03 & 4.6 \mathrm{E}-03 & 3.2 \mathrm{E}-03 & 4.5 \mathrm{E}-03 & 4.9 \mathrm{E}-03 \\ \text { TOTAL } & 6.5 \mathrm{E}-03 & 6.5 \mathrm{E}-03 & 6.6 \mathrm{E}-03 & 4.5 \mathrm{E}-03 & 6.5 \mathrm{E}-03 & 7.1 \mathrm{E}-03\end{array}$

Production/Consumption factors:

Produce: $0.41 \quad$ Milk: $2.8 \quad$ Meat: 5.8

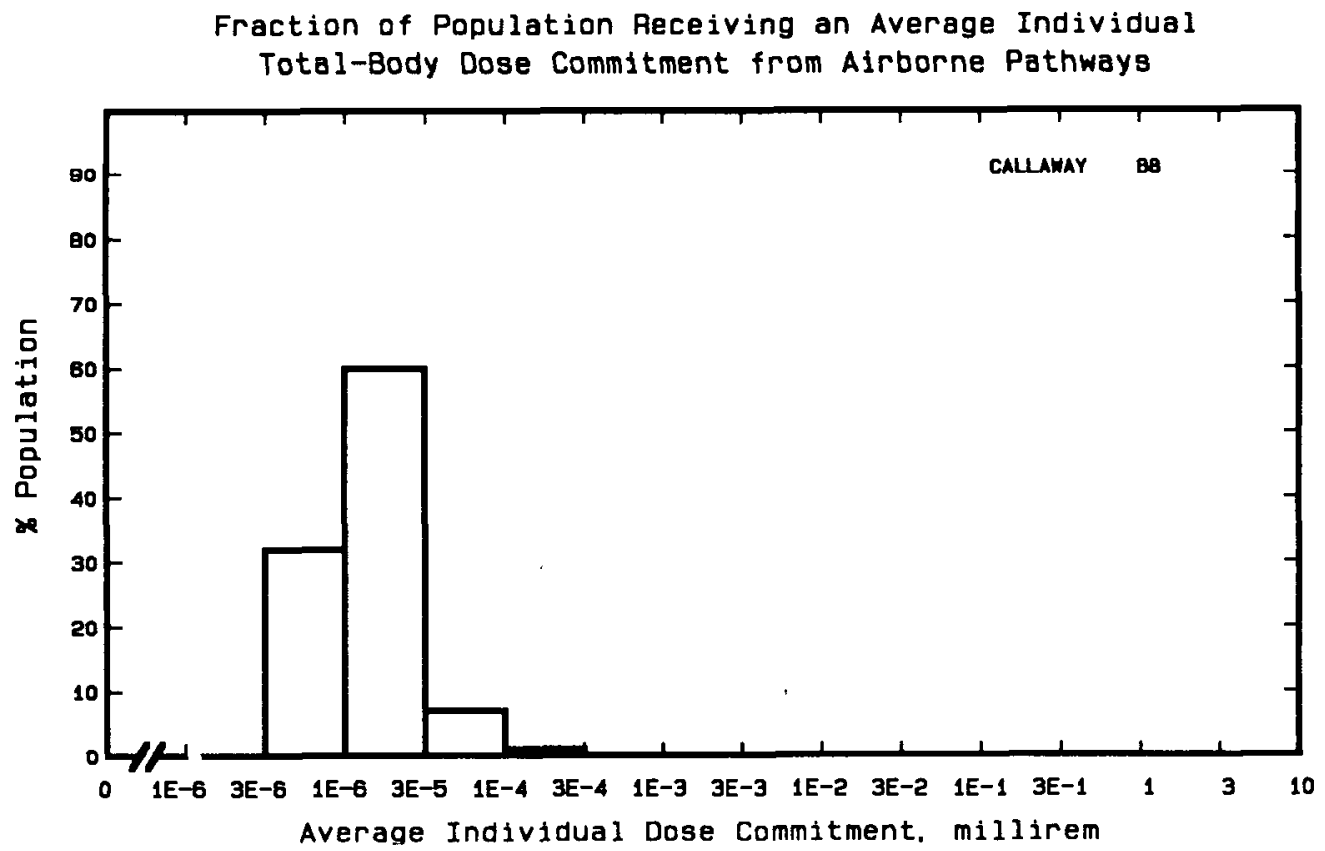


Site: CALVERT CLIFFS

LUSBY, MARYLAND

Location: N $38.4347^{\circ} \quad$ W $76.4419^{\circ}$

POPULATION DATA

Total Population Within 2-to-80-km Region: 2.8E6

Major Metropolitan Centers Within Region:

Center

Washington, DC SMSA (3/4) 2,700,000

Bowie

Annapolis

Waldorf

Sal isbury

Cambridge
Population

35,000

33,000

10,800

16,000

12,000
Location

$73 \mathrm{~km} \quad \mathrm{NW}$

$71 \mathrm{~km} \quad \mathrm{NNW}$

$61 \mathrm{~km} \mathrm{~N}$

$46 \mathrm{~km} \quad$ WNW

$75 \mathrm{~km} \quad \mathrm{E}$

$32 \mathrm{~km}$ ENE

SITE-SPECIFIC DATA - AIRBORNE PATHWAYS

Average Annual State Production

of Crops and Animal Products

In $80-\mathrm{km}$ Radius Circle

Regional Productivity Factor:

Animal Grazing Factor:
Veg: $4.5 E 7$ kilogram

Milk: 5.0E8 liter

Meat: $6.2 \mathrm{E} 7 \mathrm{kilogram}$

0.6

0.6

Meteorology Period of Record: 1 JAN 75 - 31 DEC 75 Recovery: 96\%

Fish:

Invertebrates:
Average Dilution Flow

from Plant: $2,700 \mathrm{ft}^{3} / \mathrm{s}$

Edible Harvest: $1.0 \mathrm{E} 7 \mathrm{~kg} / \mathrm{yr}$
Dilution Factor: $0.062(\mathrm{~g})$

Edible Harvest: $7.4 \mathrm{E} 6 \mathrm{~kg} / \mathrm{yr}$

Dilution Factor: 0.062 (a)

(a) Dilutions given in FES (1973). 


\section{POPULATION DOSE-COMMITMENT ESTIMATES AND AVERAGE INDIVIDUAL DOSE-COMMITMENT HISTOGRAM FOR}

CALVERT CLIFFS 1 AND 2

Dose Commitments (person-rem) from Waterborne Pathways

Total Body GI-LLI $\underline{\text { Thyroid Bone }}$

$\begin{array}{llllll}\text { Infant } & 0.0 \mathrm{E}+00 & 0.0 \mathrm{E}+00 & 0.0 \mathrm{E}+00 & 0.0 \mathrm{E}+00 & 0.0 \mathrm{E}+00 \\ \text { Child } & 7.9 \mathrm{E}-02 & 4.2 \mathrm{E}-01 & 3.1 \mathrm{E}-01 & 3.0 \mathrm{E}-01 & 3.5 \mathrm{E}-01 \\ \text { Teen } & 1.3 \mathrm{E}-01 & 8.8 \mathrm{E}-01 & 2.2 \mathrm{E}-01 & 1.8 \mathrm{E}-01 & 2.9 \mathrm{E}-01 \\ \text { Adult } & 1.3 \mathrm{E}+00 & 7.7 \mathrm{E}+00 & 1.4 \mathrm{E}+00 & 1.0 \mathrm{E}+00 & 1.7 \mathrm{E}+00 \\ \text { TOTAL } & 1.5 \mathrm{E}+00 & 9.0 \mathrm{E}+00 & 2.0 \mathrm{E}+00 & 1.5 \mathrm{E}+00 & 2.4 \mathrm{E}+00\end{array}$

Dose Commitments (person-rem) from Airborne Pathways

Total Body GI-LLI Thyroid Bone Liver Lung

$\begin{array}{lllllll}\text { Infant } & 3.7 \mathrm{E}-03 & 3.3 \mathrm{E}-03 & 1.8 \mathrm{E}-01 & 5.2 \mathrm{E}-03 & 6.0 \mathrm{E}-03 & 3.8 \mathrm{E}-03 \\ \text { Child } & 4.0 \mathrm{E}-02 & 3.7 \mathrm{E}-02 & 9.4 \mathrm{E}-01 & 4.9 \mathrm{E}-02 & 5.3 \mathrm{E}-02 & 4.2 \mathrm{E}-02 \\ \text { Teen } & 2.9 \mathrm{E}-02 & 2.7 \mathrm{E}-02 & 3.8 \mathrm{E}-01 & 2.9 \mathrm{E}-02 & 3.3 \mathrm{E}-02 & 3.2 \mathrm{E}-02 \\ \text { Adult } & 1.7 \mathrm{E}-01 & 1.6 \mathrm{E}-01 & 1.3 \mathrm{E}+00 & 1.6 \mathrm{E}-01 & 1.8 \mathrm{E}-01 & 1.7 \mathrm{E}-01 \\ \text { TOTAL } & 2.4 \mathrm{E}-01 & 2.3 \mathrm{E}-01 & 2.8 \mathrm{E}+00 & 2.4 \mathrm{E}-01 & 2.7 \mathrm{E}-01 & 2.5 \mathrm{E}-01\end{array}$

Production/Consumption factors:

Produce: $0.049 \quad$ Milk: $0.80 \quad$ Meat: 0.16

Fraction of Population Receiving an Average Individual Total-Body Dose Commitment from Airborne Pathways

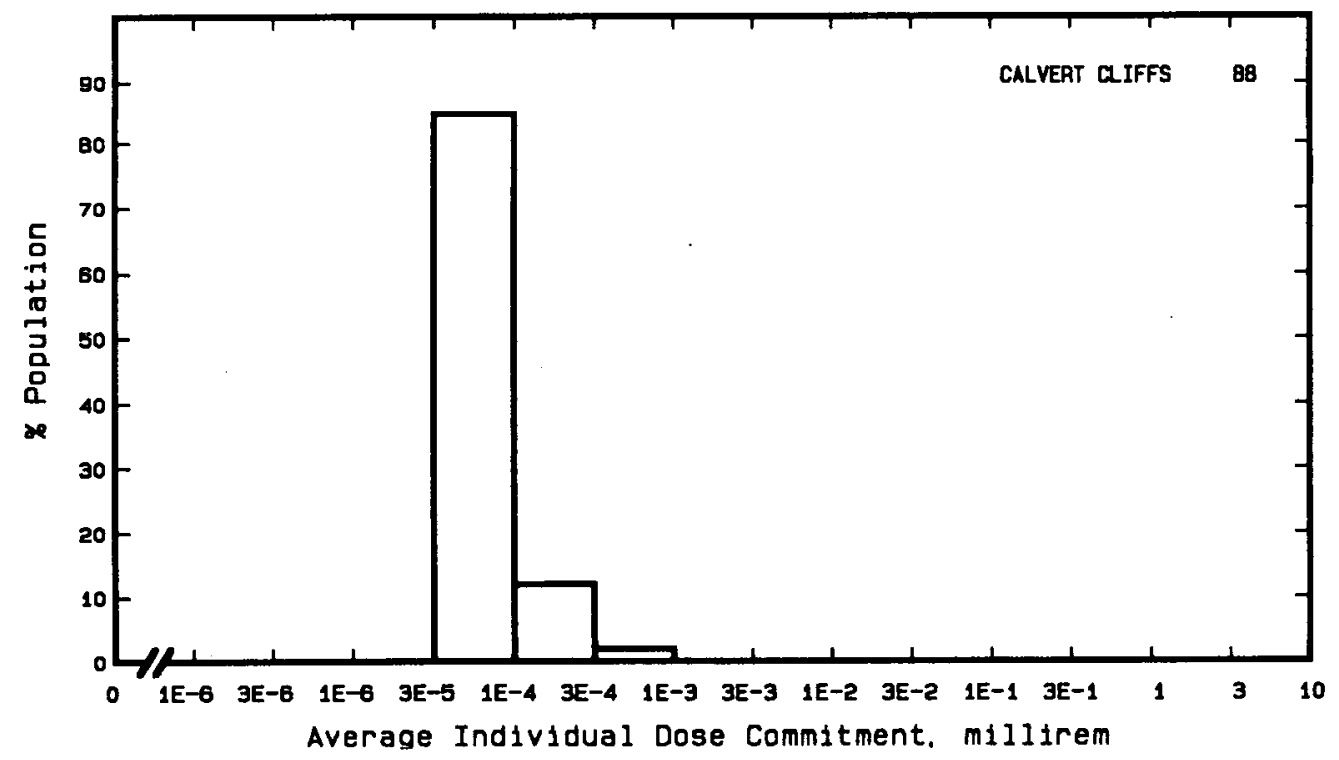


Site: CATAWBA

CLOVER, SOUTH CAROLINA

Location: $\quad$ N $34.9950^{\circ} \quad$ W $81.2450^{\circ}$

POPULATION DATA

Total Population Within 2-to-80-km Region: 1.6E6

Major Metropolitan Centers Within Region:

Center Population Location

$\begin{array}{llll}\text { Charlotte-Gastonia SMSA } \quad 680,000 & 29 \mathrm{~km} & \text { NE }\end{array}$

Kannapolis $\quad 37,000 \quad 64 \mathrm{~km}$ NE

$\begin{array}{lll}\text { Rock Hill } & 37,000 & 11 \mathrm{~km} \mathrm{~S}\end{array}$

$\begin{array}{llll}\text { Spartanburg } & 16,000 & 80 \mathrm{~km}\end{array}$

SITE-SPECIFIC DATA - AIRBORNE PATHWAYS

Average Annual State Production

of Crops and Animal Products

In $80-\mathrm{km}$ Radius Circle

Veg: 7.4E6 kilogram

Milk: 5.7E7 liter

Meat: $5.0 \mathrm{E} 7 \mathrm{kilogram}$

Regional Productivity Factor:

0.9

Animal Grazing Factor:

0.75

Meteorology Period of Record: 17 DEC 75 - 16 DEC 76 Recovery: 94\%

SITE-SPECIFIC DATA - WATERBORNE PATHWAYS via CATAWBA RIVER

Average River Flow

at Site: $4,400 \mathrm{ft}^{3} / \mathrm{s}$

Drinking Water:

None

Fish:

Edible Harvest: $1.0 \mathrm{E} 6 \mathrm{~kg} / \mathrm{yr}$

Dilution Factor: 1 
POPULATION DOSE-COMMITMENT ESTIMATES AND

AVERAGE INDIVIDUAL DOSE-COMMITMENT HISTOGRAM FOR

CATAWBA 1 AND 2

Dose Commitments (person-rem) from Waterborne Pathways

Total Body GI-LLI Ihyroid Bone

$\begin{array}{llllll}\text { Infant } & 0.0 \mathrm{E}+00 & 0.0 \mathrm{E}+00 & 0.0 \mathrm{E}+00 & 0.0 \mathrm{E}+00 & 0.0 \mathrm{E}+00 \\ \text { Child } & 6.2 \mathrm{E}-02 & 9.6 \mathrm{E}-02 & 1.6 \mathrm{E}-02 & 2.8 \mathrm{E}-01 & 3.3 \mathrm{E}-01 \\ \text { Teen } & 1.1 \mathrm{E}-01 & 2.0 \mathrm{E}-01 & 1.2 \mathrm{E}-02 & 1.7 \mathrm{E}-01 & 2.8 \mathrm{E}-01 \\ \text { Adult } & 1.2 \mathrm{E}+00 & 1.7 \mathrm{E}+00 & 8.1 \mathrm{E}-02 & 9.6 \mathrm{E}-01 & 1.7 \mathrm{E}+00 \\ \text { TOTAL } & 1.4 \mathrm{E}+00 & 2.0 \mathrm{E}+00 & 1.1 \mathrm{E}-01 & 1.4 \mathrm{E}+00 & 2.3 \mathrm{E}+00\end{array}$

Dose Commitments (person-rem) from Airborne Pathways

Total Body GI-LLI Thyroid Bone Liver Lung

$\begin{array}{lllllll}\text { Infant } & 2.8 \mathrm{E}-03 & 2.8 \mathrm{E}-03 & 3.5 \mathrm{E}-03 & 2.5 \mathrm{E}-03 & 2.8 \mathrm{E}-03 & 3.0 \mathrm{E}-03 \\ \text { Child } & 3.3 \mathrm{E}-02 & 3.3 \mathrm{E}-02 & 3.8 \mathrm{E}-02 & 2.7 \mathrm{E}-02 & 3.3 \mathrm{E}-02 & 3.6 \mathrm{E}-02 \\ \text { Teen } & 2.4 \mathrm{E}-02 & 2.5 \mathrm{E}-02 & 2.7 \mathrm{E}-02 & 2.0 \mathrm{E}-02 & 2.5 \mathrm{E}-02 & 2.9 \mathrm{E}-02 \\ \text { Aduit } & 1.5 \mathrm{E}-01 & 1.5 \mathrm{E}-01 & 1.6 \mathrm{E}-01 & 1.2 \mathrm{E}-01 & 1.5 \mathrm{E}-01 & 1.6 \mathrm{E}-01 \\ \text { TOTAL } & 2.1 \mathrm{E}-01 & 2.1 \mathrm{E}-01 & 2.3 \mathrm{E}-01 & 1.7 \mathrm{E}-01 & 2.1 \mathrm{E}-01 & 2.3 \mathrm{E}-01\end{array}$

Production/Consumption factors:

Produce: $0.022 \quad$ Milk: 0.25 Meat: 0.35

Fraction of Population Receiving an Average Individual Total-Body Dose Commitment from Airborne Pathways

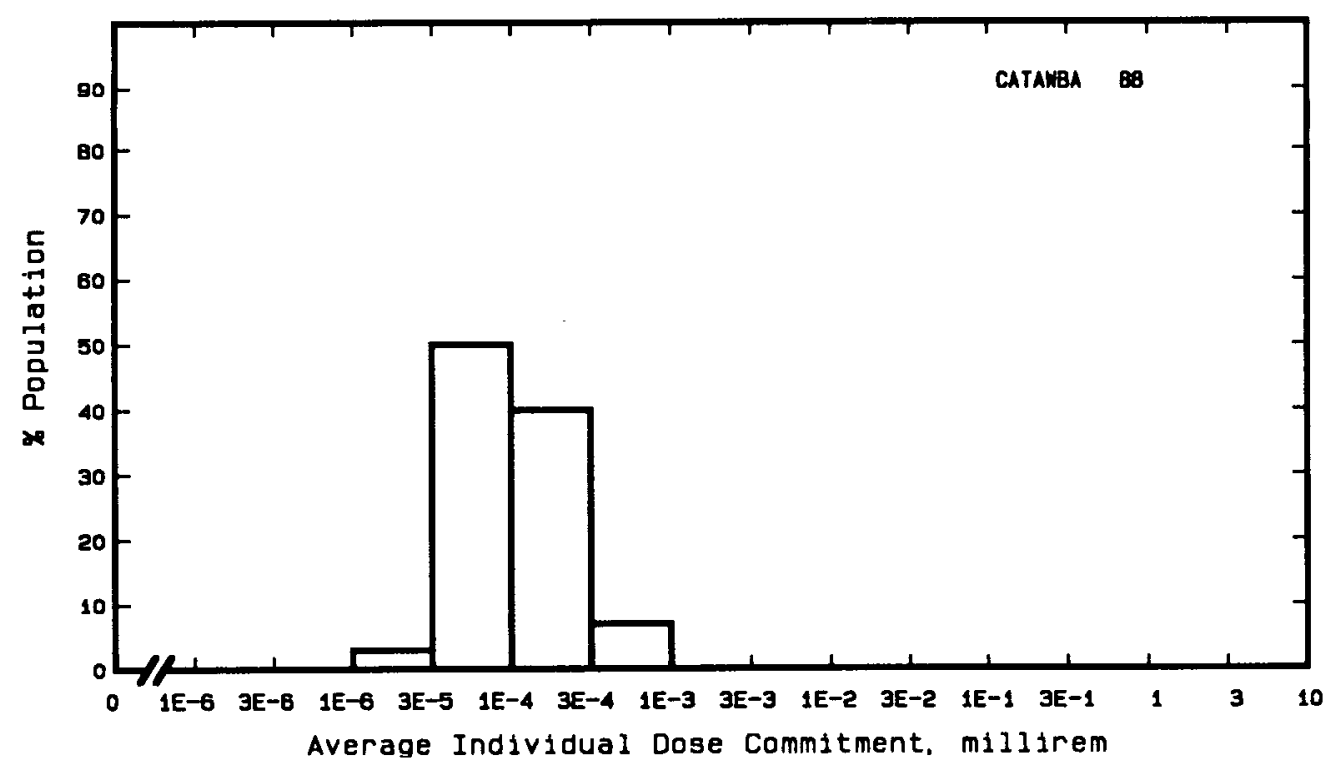


Site: CLINTON

CLINTON, ILLINOIS

Location: $\quad$ N $40.1517^{\circ} \quad$ W $88.9533^{\circ}$

POPULATION DATA

Total Population Within 2-to-80-km Region: 2.7E6

Major Metropolitan Centers Within Region:

Center

Decatur

Springfield

Champaign-Urbana

Bloomington-Normal
Population

94,000

100,000

94,000

80,000
Location

$32 \mathrm{~km} \mathrm{~S}$

$72 \mathrm{~km} \mathrm{SW}$

$50 \mathrm{~km} \quad \mathrm{E}$

$35 \mathrm{~km} \quad \mathrm{NNW}$

\section{SITE-SPECIFIC DATA - AIRBORNE PATHWAYS}

Average Annual State Production

of Crops and Animal Products

In $80-\mathrm{km}$ Radius Circle

Regional Productivity Factor:

Animal Grazing Factor:
Veg: 1.1 E8 kilogram

Mi1k: 1.8E8 liter

Meat: 1.9 E8 kilogram

0.9

0.5

Meteorology Period of Record: 14 MAR 72 - 28 SEP 79 Recovery: $91 \%$

Average Dilution Flow from $\mathrm{Pl}$ ant: $0.38 \mathrm{ft}^{3} / \mathrm{s}$

Drinking Water:

None

Fish:

Edible Harvest: $0.18^{(\mathrm{a})} \mathrm{kg} / \mathrm{yr}$ Dilution Factor: 0.25

(a) Ten percent of population are assumed to obtain fish from lake. Average individual consumption rate of $5 \mathrm{~g} / \mathrm{d}$ given in FES (1974) used in lieu of catch data. 
Dose Commitments (person-rem) from Waterborne Pathways Total Body GI-LLI Thyroid Bone Liver

$\begin{array}{llllll}\text { Infant } & 0.0 \mathrm{E}+00 & 0.0 \mathrm{E}+00 & 0.0 \mathrm{E}+00 & 0.0 \mathrm{E}+00 & 0.0 \mathrm{E}+00 \\ \text { Child } & 6.4 \mathrm{E}-01 & 1.5 \mathrm{E}+00 & 1.3 \mathrm{E}-02 & 1.5 \mathrm{E}+00 & 1.3 \mathrm{E}+00 \\ \text { Teen } & 4.6 \mathrm{E}-01 & 3.4 \mathrm{E}+00 & 1.1 \mathrm{E}-02 & 9.6 \mathrm{E}-01 & 1.2 \mathrm{E}+00 \\ \text { Adult } & 2.9 \mathrm{E}+00 & 3.1 \mathrm{E}+01 & 9.0 \mathrm{E}-02 & 6.1 \mathrm{E}+00 & 7.5 \mathrm{E}+00 \\ \text { TOTAL } & 4.0 \mathrm{E}+00 & 3.5 \mathrm{E}+01 & 1.1 \mathrm{E}-01 & 8.5 \mathrm{E}+00 & 1.0 \mathrm{E}+01\end{array}$

Dose Commitments (person-rem) from Airborne Pathways

Total Body GI-LLI Thyroid Bone Liver Lung

$\begin{array}{lllllll}\text { Infant } & 9.7 \mathrm{E}-05 & 9.7 \mathrm{E}-05 & 2.1 \mathrm{E}-04 & 3.9 \mathrm{E}-05 & 9.7 \mathrm{E}-05 & 1.5 \mathrm{E}-04 \\ \text { Child } & 1.6 \mathrm{E}-03 & 1.6 \mathrm{E}-03 & 2.4 \mathrm{E}-03 & 4.7 \mathrm{E}-04 & 1.5 \mathrm{E}-03 & 2.4 \mathrm{E}-03 \\ \text { Teen } & 1.0 \mathrm{E}-03 & 1.2 \mathrm{E}-03 & 1.4 \mathrm{E}-03 & 3.2 \mathrm{E}-04 & 1.0 \mathrm{E}-03 & 1.9 \mathrm{E}-03 \\ \text { Adult } & 6.0 \mathrm{E}-03 & 7.0 \mathrm{E}-03 & 7.5 \mathrm{E}-03 & 1.9 \mathrm{E}-03 & 6.0 \mathrm{E}-03 & 9.5 \mathrm{E}-03 \\ \text { TOTAL } & 8.7 \mathrm{E}-03 & 9.9 \mathrm{E}-03 & 1.2 \mathrm{E}-02 & 2.8 \mathrm{E}-03 & 8.7 \mathrm{E}-03 & 1.4 \mathrm{E}-02\end{array}$

Production/Consumption factors:

Produce: $0.19 \quad$ Milk: $0.45 \quad$ Meat: 0.78

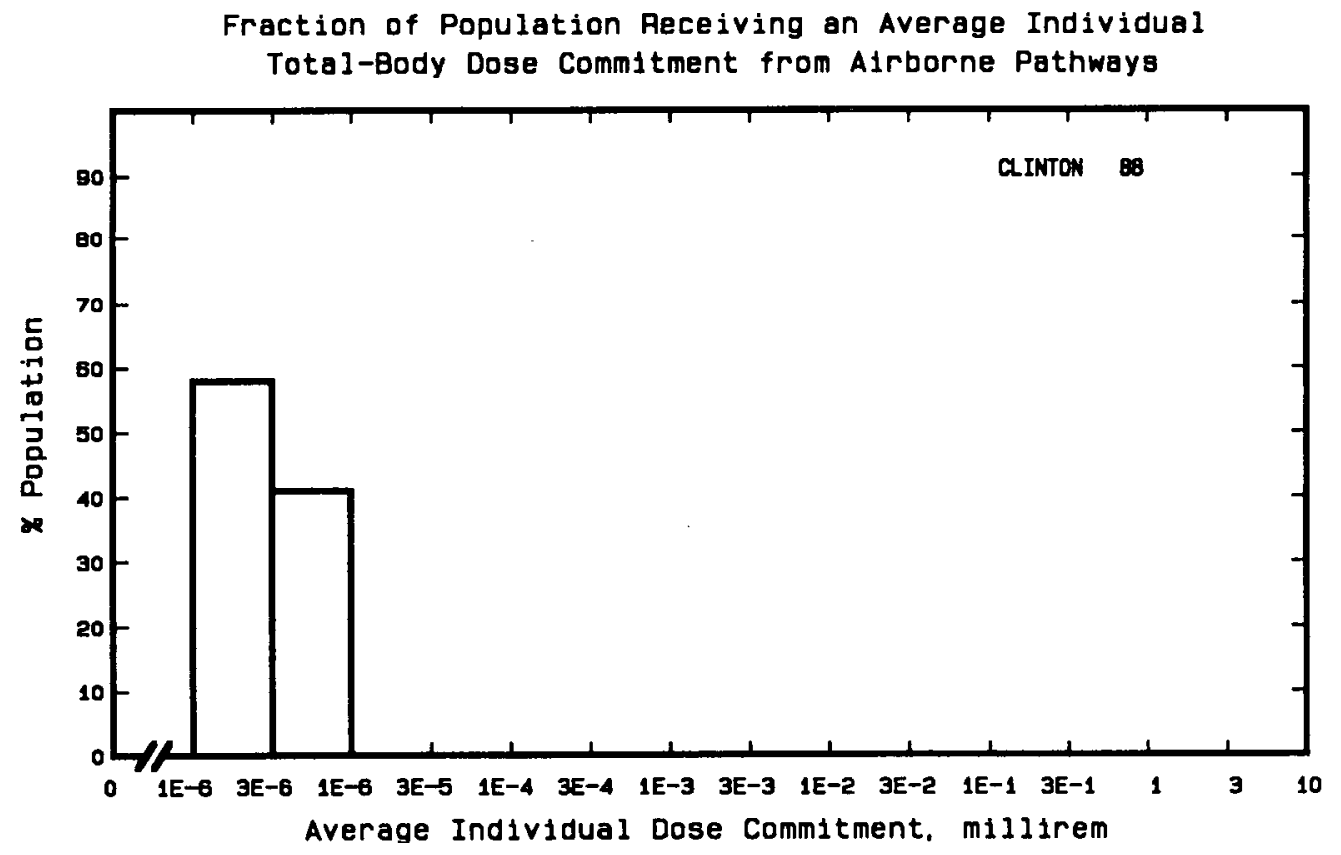


Site: COOK

BENTON HARBOR, MICHIGAN

Location: $\quad$ N $41.9761^{\circ} \quad$ W $86.5664^{\circ}$

POPULATION DATA

Total Population Within 2-to-80-km Region: $1.2 \mathrm{E} 6$

Major Metropolitan Centers Within Region:

Center

Gary

South Bend SMSA

Elkhart SMSA

Mishiwaka

Michigan City
Population

140,000

280,000

140,000

40,000

37,000

\section{Location}

$77 \mathrm{~km}$. SW

$42 \mathrm{~km} \quad \mathrm{SE}$

$58 \mathrm{~km} \quad \mathrm{SE}$

$47 \mathrm{~km} \quad \mathrm{SE}$

$40 \mathrm{~km} \quad \mathrm{SW}$

SITE-SPECIFIC DATA - AIRBORNE PATHWAYS

Average Annual State Production

of Crops and Animal Products

In $80-\mathrm{km}$ Radius Circle

Regional Productivity Factor:

Animal Grazing Factor:
Veg: $1.1 \mathrm{E8}$ kilogram

Mi1k: 2.3E8 liter

Meat: $1.9 \mathrm{E} 8$ kilogram

0.6

0.5

Meteorology Period of Record: 1 MAY 75 - 31 APR 76 Recovery: 95\%

Drinking Water:

Fish:

\section{via LAKE MICHIGAN}

Average Dilution Flow

from Plant: $2,500 \mathrm{ft}^{3} / \mathrm{s}$

Exposed Population: 260,000

Dilution Factor: $0.025(a)$

Edible Harvest: $1.5 \mathrm{E} 6 \mathrm{~kg} / \mathrm{yr}$

Dilution Factor: 0.01

(a) Drinking water dilution factor est imated by averaging dilution factors derived from FES (1973) suitably weighted for population. 
POPULATION DOSE-COMMITMENT ESTIMATES AND

AVERAGE INDIVIDUAL DOSE-COMMITMENT HISTOGRAM FOR

COOK 1 AND 2

Dose Commitments (person-rem) from Waterborne Pathways

Total Body

Infant

Child

Teen

Adult

TOTAL
2. $4 \mathrm{E}-03$

$3.1 \mathrm{E}-02$

$1.7 \mathrm{E}-02$

$1.6 \mathrm{E}-01$

2. $1 E-01$
$\underline{G I-L L I}$

$2.4 \mathrm{E}-03$

2.8E-02

$1.2 \mathrm{E}-02$

$1.0 \mathrm{E}-01$

$1.4 \mathrm{E}-01$
Thyroid

$5.9 \mathrm{E}-03$

$5.2 \mathrm{E}-02$

$1.8 \mathrm{E}-02$

$1.4 \mathrm{E}-01$

2.2E-01

Bone

Liver

Dose Commitments (person-rem) from Airborne Pathways

Total Body GI-LLI Thyroid Bone Liver Lung

$\begin{array}{lllllll}\text { Infant } & 7.8 \mathrm{E}-05 & 6.7 \mathrm{E}-05 & 3.6 \mathrm{E}-03 & 1.3 \mathrm{E}-04 & 1.7 \mathrm{E}-04 & 7.9 \mathrm{E}-05 \\ \text { Child } & 9.7 \mathrm{E}-04 & 8.1 \mathrm{E}-04 & 2.4 \mathrm{E}-02 & 1.4 \mathrm{E}-03 & 1.7 \mathrm{E}-03 & 9.4 \mathrm{E}-04 \\ \text { Teen } & 7.1 \mathrm{E}-04 & 5.7 \mathrm{E}-04 & 9.4 \mathrm{E}-03 & 6.7 \mathrm{E}-04 & 9.3 \mathrm{E}-04 & 6.7 \mathrm{E}-04 \\ \text { Adult } & 4.2 \mathrm{E}-03 & 3.4 \mathrm{E}-03 & 3.3 \mathrm{E}-02 & 3.3 \mathrm{E}-03 & 4.5 \mathrm{E}-03 & 3.7 \mathrm{E}-03 \\ \text { TOTAL } & 5.9 \mathrm{E}-03 & 4.8 \mathrm{E}-03 & 7.0 \mathrm{E}-02 & 5.5 \mathrm{E}-03 & 7.2 \mathrm{E}-03 & 5.4 \mathrm{E}-03\end{array}$

Production/Consumption factors:

Produce: 0.29

Milk: 0.91

Meat: 1.2

Fraction of Population Receiving an Average Individual Total-Body Dose Commitment from Airborne Pathways

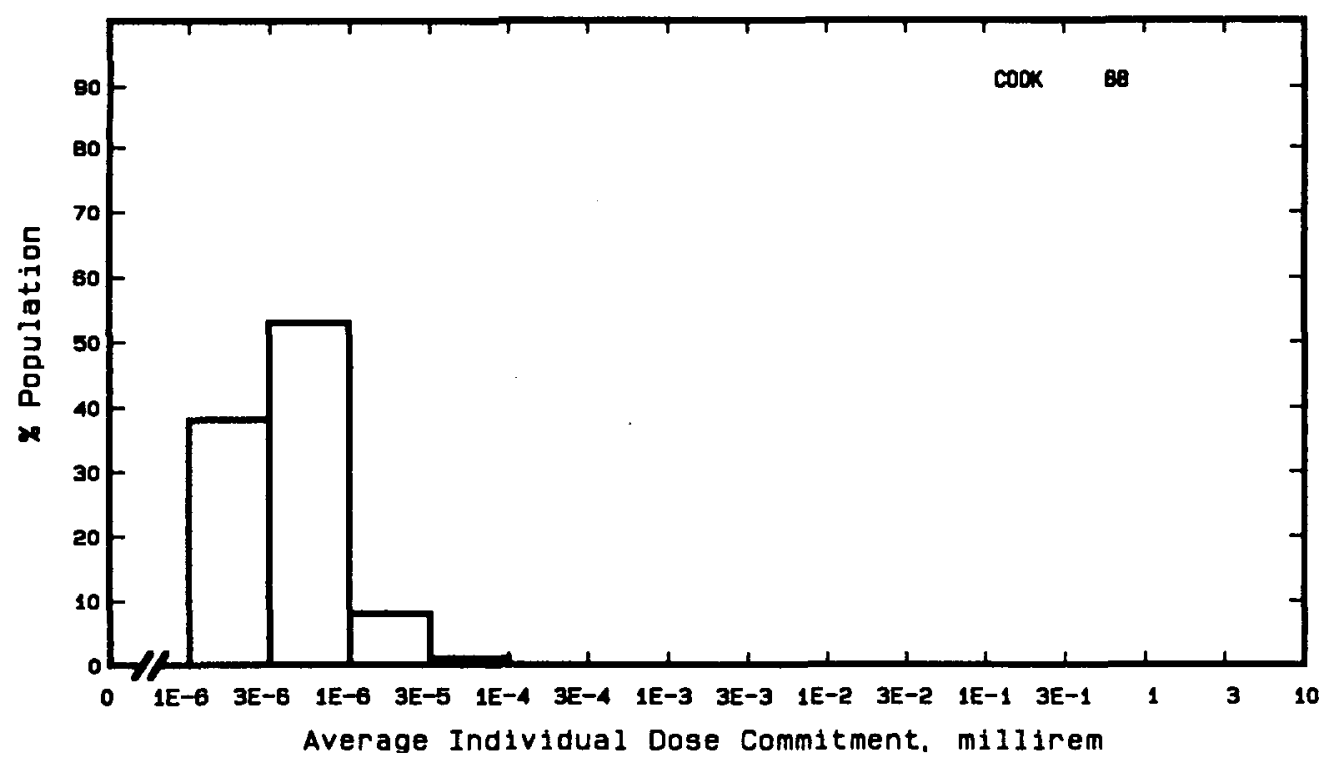


Site: COOPER

NEMAHA COUNTY, NEBRASKA

Location: $\quad N 40.3619^{\circ} \quad$ W $95.6411^{\circ}$

POPULATION DATA

Total Population Within 2-to-80-km Region: 1.7E5

Major Metropolitan Centers Within Region:

Center

Nebraska City

Red Oak

Platt smouth

Shenandoah

Falls City
Population

7,100

6,800

6,300

6,300

5,400
Location

$40 \mathrm{~km} \quad \mathrm{NNW}$

$80 \mathrm{~km} \quad \mathrm{NNE}$

$76 \mathrm{~km} \quad \mathrm{NNW}$

$51 \mathrm{~km} \quad \mathrm{NNE}$

$33 \mathrm{~km} \mathrm{~S}$

\section{SITE-SPECIFIC DATA - AIRBORNE PATHWAYS}

Average Annual State Production

of Crops and Animal Products

In $80-\mathrm{km}$ Radius Circle

Regional Productivity Factor:

Animal Grazing Factor:
Veg: $9.7 \mathrm{E} 7 \mathrm{kilogram}$

Milk: 7.2E7 liter

Meat: 2.0E8 kilogram

1

0.6

Meteorology Period of Record: I MAR 70 - 31 DEC 75 Recovery: $89 \%$

SITE-SPECIFIC DATA - WATERBORNE PATHWAYS via MISSOURI RIVER

Average River Flow

at Site: $31,000 \mathrm{ft}^{3} / \mathrm{s}$

Drinking Water:

None

Fish:

Edible Harvest: $5.0 \mathrm{E} 3(\mathrm{a}) \mathrm{kg} / \mathrm{yr}$

Dilution Factor: $0.5(a)$

(a) Assumes $1 / 2 \mathrm{f}$ ish harvest caught be low plant. 


\section{POPULATION DOSE-COMMITMENT ESTIMATES AND \\ AVERAGE INDIVIDUAL DOSE-COMMITMENT HISTOGRAM FOR \\ COOPER}

Dose Commitments (person-rem) from Waterborne Pathways

Total Body GI-LLI $\underline{\text { Thyroid }}$ Bone Liver

$\begin{array}{llllll}\text { Infant } & 0.0 \mathrm{E}+00 & 0.0 \mathrm{E}+00 & 0.0 \mathrm{E}+00 & 0.0 \mathrm{E}+00 & 0.0 \mathrm{E}+00 \\ \text { Child } & 2.9 \mathrm{E}-04 & 2.5 \mathrm{E}-05 & 1.8 \mathrm{E}-05 & 1.4 \mathrm{E}-03 & 1.6 \mathrm{E}-03 \\ \text { Teen } & 5.5 \mathrm{E}-04 & 5.2 \mathrm{E}-05 & 1.3 \mathrm{E}-05 & 8.6 \mathrm{E}-04 & 1.4 \mathrm{E}-03 \\ \text { Adult } & 6.0 \mathrm{E}-03 & 4.5 \mathrm{E}-04 & 8.3 \mathrm{E}-05 & 5.0 \mathrm{E}-03 & 8.2 \mathrm{E}-03 \\ \text { TOTAL } & 6.8 \mathrm{E}-03 & 5.3 \mathrm{E}-04 & 1.1 \mathrm{E}-04 & 7.3 \mathrm{E}-03 & 1.1 \mathrm{E}-02\end{array}$

Dose Commitments (person-rem) from Airborne Pathways

Total Body GI-LLI Ihyroid Bone Liver Lung

$\begin{array}{lllllll}\text { Infant } & 7.2 \mathrm{E}-05 & 7.0 \mathrm{E}-05 & 1.4 \mathrm{E}-03 & 7.5 \mathrm{E}-05 & 7.4 \mathrm{E}-05 & 7.3 \mathrm{E}-05 \\ \text { Child } & 8.0 \mathrm{E}-04 & 7.9 \mathrm{E}-04 & 1.3 \mathrm{E}-02 & 9.6 \mathrm{E}-04 & 8.2 \mathrm{E}-04 & 8.2 \mathrm{E}-04 \\ \text { Teen } & 5.8 \mathrm{E}-04 & 5.8 \mathrm{E}-04 & 5.2 \mathrm{E}-03 & 6.2 \mathrm{E}-04 & 5.9 \mathrm{E}-04 & 6.2 \mathrm{E}-04 \\ \text { Adult } & 3.5 \mathrm{E}-03 & 3.5 \mathrm{E}-03 & 2.0 \mathrm{E}-02 & 3.6 \mathrm{E}-03 & 3.5 \mathrm{E}-03 & 3.6 \mathrm{E}-03 \\ \text { TOTAL } & 4.9 \mathrm{E}-03 & 4.9 \mathrm{E}-03 & 3.9 \mathrm{E}-02 & 5.3 \mathrm{E}-03 & 5.0 \mathrm{E}-03 & 5.1 \mathrm{E}-03\end{array}$

Production/Consumption factors:

Produce: 2.9 Milk: $3.2 \quad$ Meat: 14

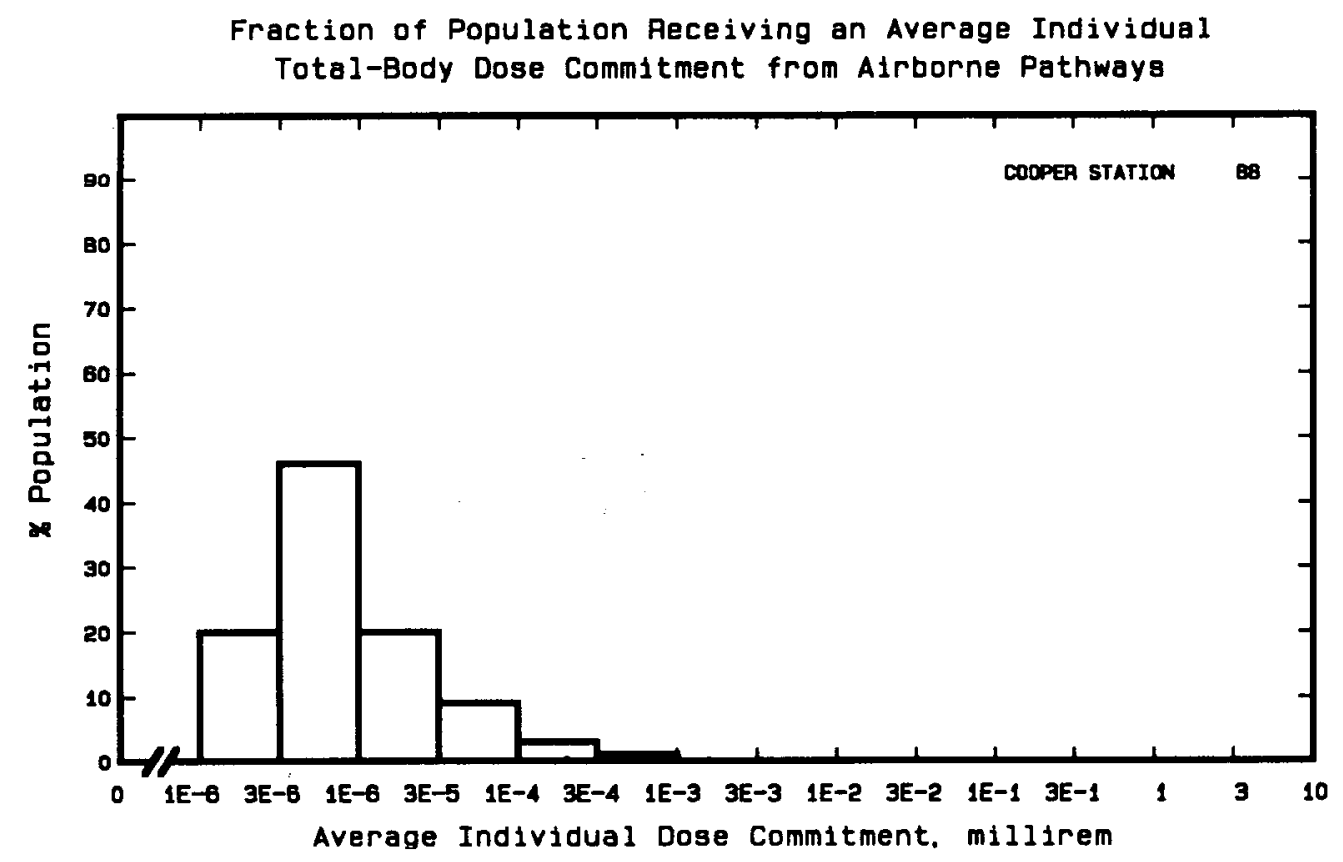


Site: CRYSTAL RIVER

CRYSTAL RIVER, FLORIDA

Location: $\quad$ N $28.9572^{\circ} \quad$ W $82.6989^{\circ}$

POPULATION DATA

Total Population Within 2-to-80-km Region: 4.6E5

Major Metropolitan Centers Within Region:

Center

Ocala

Leesburg

New Port Richey
Population

47,000

16,000

14,000
Location

$60 \mathrm{~km} \quad \mathrm{ENE}$

$80 \mathrm{~km} \quad \mathrm{E}$

$79 \mathrm{~km} \quad \mathrm{~S}$

SITE-SPECIFIC DATA - AIRBORNE PATHWAYS

Average Annual State Production

of Crops and Animal Products

In $80-\mathrm{km}$ Radius Circle

Regional Productivity Factor:

Animal Grazing Factor:

Meteorology Period of Record: 1 JAN 75 - 31 DEC 75 Recovery: 93\%
Veg: $2.8 \mathrm{E} 7 \mathrm{kilogram}$

Milk: $1.1 \mathrm{E8}$ liter

Meat: $7.2 \mathrm{E} 7$ kilogram

0.5

1

SITE-SPECIFIC DATA - WATERBORNE PATHWAYS via GULF of MEXICO

Average Dilution Flow

from Plant: $94 \mathrm{ft}^{3} / \mathrm{s}$

Fish:

Edible Harvest: $3.2 \mathrm{E} 5 \mathrm{~kg} / \mathrm{yr}$

Dilution Factor: 0.1

Invertebrates:

Edible Harvest: $1.8 \mathrm{E} 5 \mathrm{~kg} / \mathrm{yr}$

Dilution Factor: 0.1 


\section{POPULATION DOSE-COMMITMENT ESTIMATES AND \\ AVERAGE INDIVIDUAL DOSE-COMMITMENT HISTOGRAM FOR \\ CRYSTAL RIVER}

Dose Commitments (person-rem) from Waterborne Pathways

Total Body

$\underline{G I-L L I}$

Thyroid

Bone

$\underline{\text { Liver }}$

Infant

$0.0 \mathrm{E}+00$

$0.0 E+00$

$0.0 \mathrm{E}+00$

$0.0 \mathrm{E}+00$

$0.0 \mathrm{E}+00$

Child

2.8E-02

$1.4 \mathrm{E}-01$

$1.2 \mathrm{E}-02$

$6.6 \mathrm{E}-02$

4. $6 \mathrm{E}-02$

Teen

2. $0 \mathrm{E}-02$

2. $8 \mathrm{E}-01$

$9.3 \mathrm{E}-03$

$3.7 \mathrm{E}-02$

3. $6 \mathrm{E}-02$

Adult

$1.4 \mathrm{E}-01$

2. $5 \mathrm{E}+00$

$6.5 \mathrm{E}-02$

2. $2 \mathrm{E}-01$

2. $2 \mathrm{E}-01$

TOTAL

1. $9 \mathrm{E}-01$

2. $9 \mathrm{E}+00$

8.7E-02

3. $2 \mathrm{E}-01$

3. $0 \mathrm{E}-01$

Dose Commitments (person-rem) from Airborne Pathways

Total Body GI-LLI Thyroid Bone Liver $\underline{\text { Lung }}$

$\begin{array}{lllllll}\text { Infant } & 1.6 \mathrm{E}-04 & 1.6 \mathrm{E}-04 & 6.1 \mathrm{E}-04 & 1.5 \mathrm{E}-04 & 1.6 \mathrm{E}-04 & 1.7 \mathrm{E}-04 \\ \text { Child } & 1.8 \mathrm{E}-03 & 1.8 \mathrm{E}-03 & 4.1 \mathrm{E}-03 & 1.7 \mathrm{E}-03 & 1.8 \mathrm{E}-03 & 2.0 \mathrm{E}-03 \\ \text { Teen } & 1.3 \mathrm{E}-03 & 1.3 \mathrm{E}-03 & 2.2 \mathrm{E}-03 & 1.2 \mathrm{E}-03 & 1.3 \mathrm{E}-03 & 1.5 \mathrm{E}-03 \\ \text { Adult } & 7.7 \mathrm{E}-03 & 7.7 \mathrm{E}-03 & 1.0 \mathrm{E}-02 & 7.2 \mathrm{E}-03 & 7.7 \mathrm{E}-03 & 8.5 \mathrm{E}-03 \\ \text { TOTAL } & 1.1 \mathrm{E}-02 & 1.1 \mathrm{E}-02 & 1.7 \mathrm{E}-02 & 1.0 \mathrm{E}-02 & 1.1 \mathrm{E}-02 & 1.2 \mathrm{E}-02\end{array}$

Production/Consumption factors:

Produce: 0.16

Milk: 0.93

Meat: 0.98

Fraction of Population Receiving an Average Individual

Total-Body Dose Commitment from Airborne Pathways

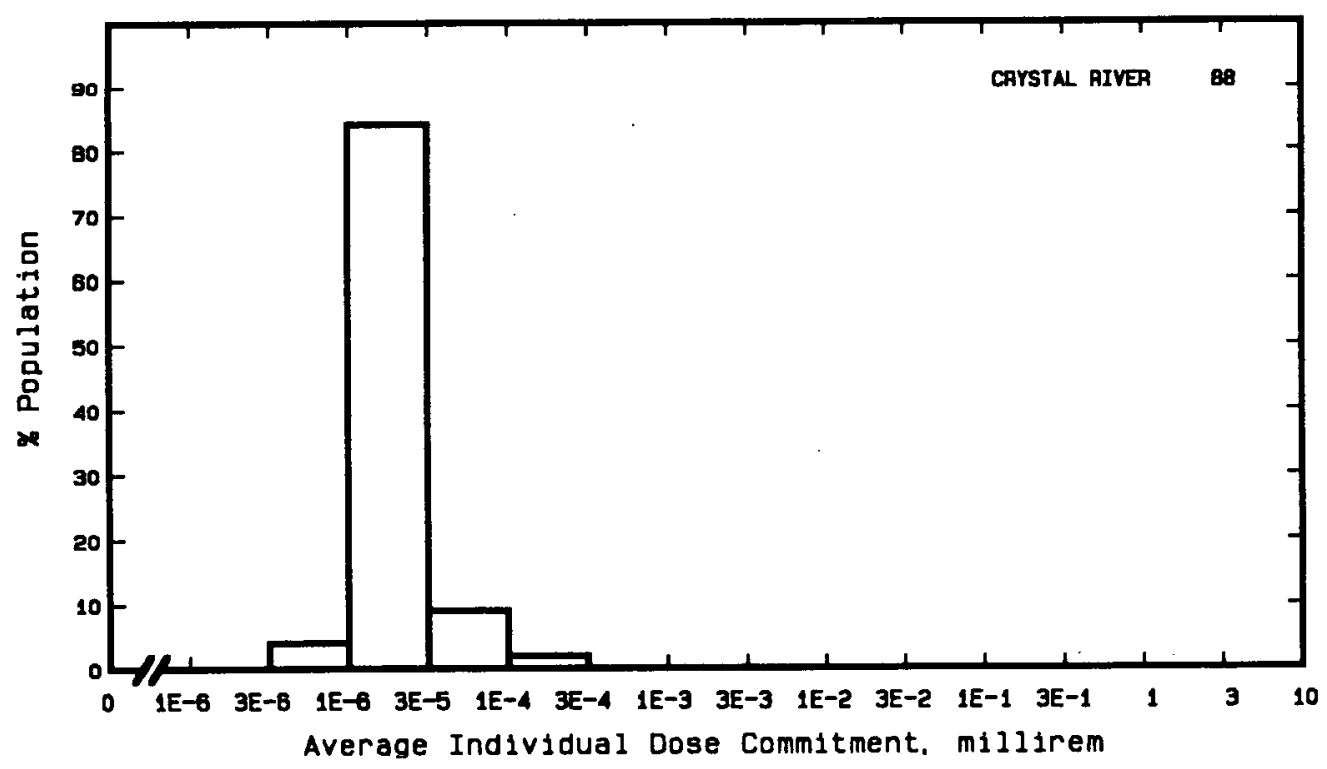


Site: DAVIS-BESSE

PORT CLINTON, OHIO

Location: N $41.5972^{\circ} \quad$ W $83.0864^{\circ}$

POPULATION DATA

Total Population Within 2-to-80-km Region: 1.8E6

Major Metropolitan Centers Within Region:

\section{Center}

Toledo SMSA

Dearborn

Taylor

Lorain

Lincoln Park

Findlay

Sandusky
Population

610,000

91,000

77,000

75,000

45,000

36,000

33,000
Location

$38 \mathrm{~km} \quad$ WNW

$80 \mathrm{~km} \mathrm{~N}$

$71 \mathrm{~km} \mathrm{~N}$

$77 \mathrm{~km} \quad$ ESE

$73 \mathrm{~km} \mathrm{~N}$

$77 \mathrm{~km} \quad \mathrm{SW}$

$35 \mathrm{~km} \quad$ ESE

\section{SITE-SPECIFIC DATA - AIRBORNE PATHWAYS}

Average Annual State Production

of Crops and Animal Products

In $80-\mathrm{km}$ Radius Circle

Regional Productivity Factor:

Animal Grazing Factor:
Veg: $6.9 \mathrm{E} 7 \mathrm{kilogram}$

Milk: 3.7E8 liter

Meat: 1.2E8 kilogram

0.6

0.5

Meteorology Period of Record: 4 AUG 74 - 3 AUG 76 Recovery: 99\%

SITE-SPECIFIC DATA - WATERBORNE PATHWAYS via LAKE ERIE

Average Dilution Flow

from Plant: $330 \mathrm{ft}^{3} / \mathrm{s}$

Drinking Water:

Exposed Population: 450,000

Dilution Factor: 1.6E-4(a)

Fish:

Edible Harvest: $5.7 \mathrm{E}{ }^{(\mathrm{b})} \mathrm{kg} / \mathrm{yr}$

Dilution Factor: $1.8 \mathrm{E}-4$ (b)

(a) Drinking water dilution factor estimated by averaging dilution factor derived from FES (1973), suitably weighted for population.

(b) Letter from Terry D. Murray, Toledo Edison Company to Charles A. Willis, NRC, July 20, 1984. 


\section{POPULATION DOSE-COMMITMENT ESTIMATES AND \\ AVERAGE INDIVIDUAL DOSE-COMMITMENT HISTOGRAM FOR \\ DAVIS-BESSE}

Dose Commitments (person-rem) from Waterborne Pathways

Total Body GI-LLI Thyroid Bone Liver

$\begin{array}{llllll}\text { Infant } & 7.8 \mathrm{E}-06 & 8.5 \mathrm{E}-06 & 8.4 \mathrm{E}-06 & 1.6 \mathrm{E}-06 & 8.1 \mathrm{E}-06 \\ \text { Child } & 1.6 \mathrm{E}-04 & 1.4 \mathrm{E}-04 & 9.0 \mathrm{E}-05 & 3.3 \mathrm{E}-04 & 4.2 \mathrm{E}-04 \\ \text { Teen } & 1.5 \mathrm{E}-04 & 1.2 \mathrm{E}-04 & 3.5 \mathrm{E}-05 & 1.9 \mathrm{E}-04 & 3.1 \mathrm{E}-04 \\ \text { Adult } & 1.5 \mathrm{E}-03 & 1.1 \mathrm{E}-03 & 2.9 \mathrm{E}-04 & 1.1 \mathrm{E}-03 & 1.9 \mathrm{E}-03 \\ \text { TOTAL } & 1.8 \mathrm{E}-03 & 1.3 \mathrm{E}-03 & 4.2 \mathrm{E}-04 & 1.6 \mathrm{E}-03 & 2.7 \mathrm{E}-03\end{array}$

Dose Commitments (person-rem) from Airborne Pathways

Total Body GI-LLI Thyroid Bone Liver Lung

$\begin{array}{lllllll}\text { Infant } & 2.5 \mathrm{E}-04 & 2.5 \mathrm{E}-04 & 7.1 \mathrm{E}-04 & 2.8 \mathrm{E}-05 & 2.5 \mathrm{E}-04 & 2.5 \mathrm{E}-04 \\ \text { Child } & 3.1 \mathrm{E}-03 & 3.1 \mathrm{E}-03 & 5.7 \mathrm{E}-03 & 3.1 \mathrm{E}-04 & 3.1 \mathrm{E}-03 & 3.1 \mathrm{E}-03 \\ \text { Teen } & 1.9 \mathrm{E}-03 & 1.9 \mathrm{E}-03 & 2.9 \mathrm{E}-03 & 2.2 \mathrm{E}-04 & 1.9 \mathrm{E}-03 & 2.0 \mathrm{E}-03 \\ \text { Adult } & 1.0 \mathrm{E}-02 & 1.0 \mathrm{E}-02 & 1.3 \mathrm{E}-02 & 1.3 \mathrm{E}-03 & 1.0 \mathrm{E}-02 & 1.0 \mathrm{E}-02 \\ \text { TOTAL } & 1.6 \mathrm{E}-02 & 1.6 \mathrm{E}-02 & 2.3 \mathrm{E}-02 & 1.9 \mathrm{E}-03 & 1.6 \mathrm{E}-02 & 1.6 \mathrm{E}-02\end{array}$

Production/Consumption factors:

Produce: $0.12 \quad$ Milk: $0.95 \quad$ Meat: 0.48

Fraction of Population Receiving an Average Individual

Tota]-Body Dose Commitment from Alrborne Pathways

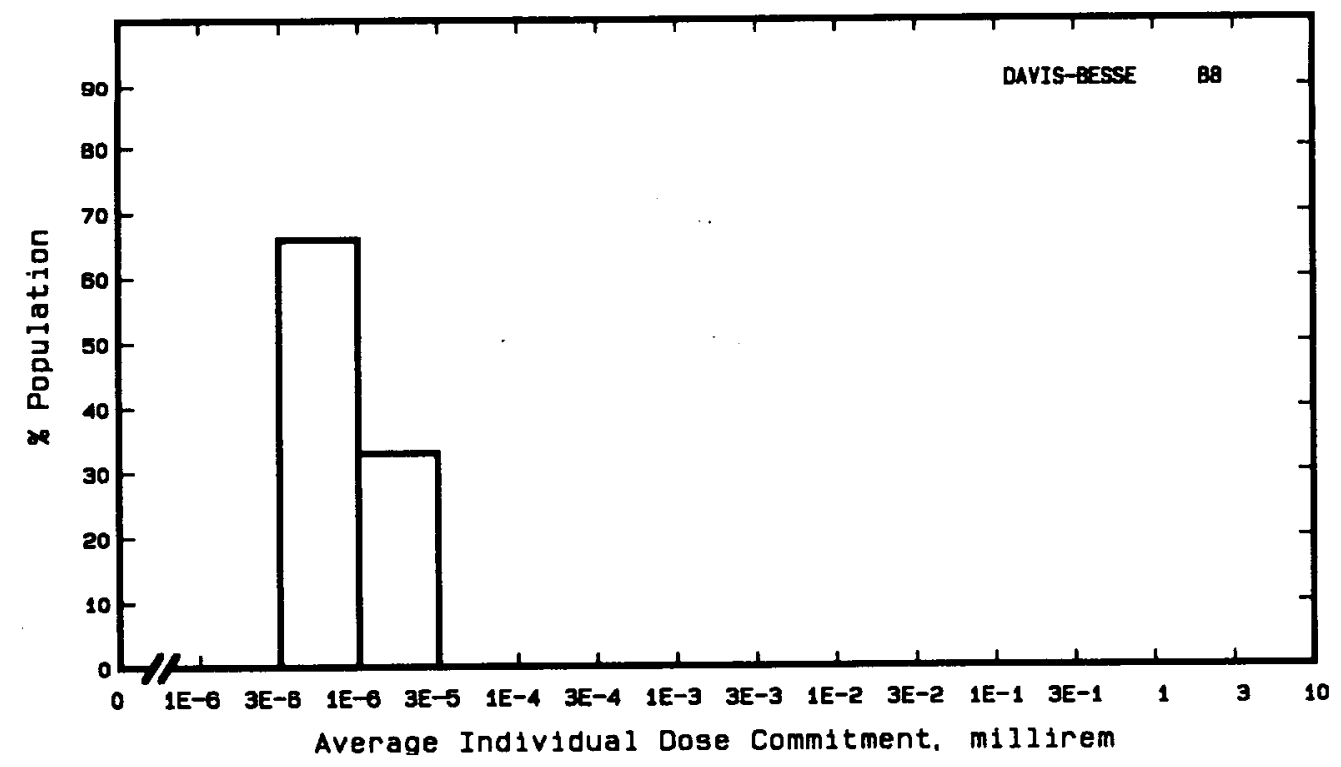


Site: DIABLO CANYON

AVILA BEACH, CALIFORNIA

Location: $\quad$ N $35.2111^{\circ} \quad$ W $120.8522^{\circ}$

POPULATION DATA

Total Population Within 2-to-80-km Region: 3.1E5

Major Metropolitan Centers Within Region:

Center

San Luis Obispo

Atascudero

Lompoc

Morro Bay
Population

40,000

14,000

28,000

10,000
Location

$19 \mathrm{~km} \quad \mathrm{ENE}$

$34 \mathrm{~km} \quad \mathrm{NNE}$

$74 \mathrm{~km} \quad$ SSE

$18 \mathrm{~km} \mathrm{~N}$

SITE-SPECIFIC DATA - AIRBORNE PATHWAYS

Average Annual State Production

of Crops and Animal Products

In $80-\mathrm{km}$ Radius Circle

Regional Productivity Factor:

Animal Grazing Factor:
Veg: $4.8 \mathrm{E} 7 \mathrm{kilogram}$

Milk: 2.3E8 liter

Meat: $5.0 \mathrm{E} 7$ kilogram

0.5

1

Meteorology Period of Record: 1 JAN 84 - 31 DEC 84 Recovery: 98\%

Fish:

Edible Harvest: $2.0 \mathrm{E} 6 \mathrm{~kg} / \mathrm{yr}$

Dilution Factor: 0.001

Invertebrates:

None 


\section{POPULATION DOSE-COMMITMENT ESTIMATES AND \\ AVERAGE INDIVIDUAL DOSE-COMMITTMENT HISTOGRAM FOR}

DIABLO CANYON 1 AND 2

Dose Commitments (person-rem) from Waterborne Pathways

Total Body GI-LLI Thyroid Bone Liver

$\begin{array}{llllll}\text { Infant } & 0.0 \mathrm{E}+00 & 0.0 \mathrm{E}+00 & 0.0 \mathrm{E}+00 & 0.0 \mathrm{E}+00 & 0.0 \mathrm{E}+00 \\ \text { Child } & 6.7 \mathrm{E}-04 & 5.9 \mathrm{E}-04 & 3.5 \mathrm{E}-04 & 2.8 \mathrm{E}-03 & 2.1 \mathrm{E}-03 \\ \text { Teen } & 6.1 \mathrm{E}-04 & 1.2 \mathrm{E}-03 & 2.6 \mathrm{E}-04 & 1.6 \mathrm{E}-03 & 1.7 \mathrm{E}-03 \\ \text { Adult } & 5.0 \mathrm{E}-03 & 9.9 \mathrm{E}-03 & 1.7 \mathrm{E}-03 & 9.3 \mathrm{E}-03 & 9.8 \mathrm{E}-03 \\ \text { TOTAL } & 6.3 \mathrm{E}-03 & 1.2 \mathrm{E}-02 & 2.3 \mathrm{E}-03 & 1.4 \mathrm{E}-02 & 1.4 \mathrm{E}-02\end{array}$

Dose Commitments (person-rem) from Airborne Pathways

Total Body GI-LLI Thyroid Bone Liver

$\begin{array}{lllllll}\text { Infant } & 1.7 \mathrm{E}-04 & 1.7 \mathrm{E}-04 & 4.1 \mathrm{E}-04 & 5.0 \mathrm{E}-05 & 1.8 \mathrm{E}-04 & 1.8 \mathrm{E}-04 \\ \text { Child } & 2.7 \mathrm{E}-03 & 2.7 \mathrm{E}-03 & 4.1 \mathrm{E}-03 & 5.6 \mathrm{E}-04 & 2.7 \mathrm{E}-03 & 2.8 \mathrm{E}-03 \\ \text { Teen } & 1.8 \mathrm{E}-03 & 1.8 \mathrm{E}-03 & 2.3 \mathrm{E}-03 & 4.0 \mathrm{E}-04 & 1.8 \mathrm{E}-03 & 1.8 \mathrm{E}-03 \\ \text { Adult } & 9.9 \mathrm{E}-03 & 9.9 \mathrm{E}-03 & 1.2 \mathrm{E}-02 & 2.4 \mathrm{E}-03 & 9.9 \mathrm{E}-03 & 1.0 \mathrm{E}-02 \\ \text { TOTAL } & 1.5 \mathrm{E}-02 & 1.5 \mathrm{E}-02 & 1.9 \mathrm{E}-02 & 3.4 \mathrm{E}-03 & 1.5 \mathrm{E}-02 & 1.5 \mathrm{E}-02\end{array}$

Production/Consumption factors:

Produce: $0.40 \quad$ Milk: $2.9 \quad$ Meat: 1.0

Fraction of Population Receiving an Average Individual

Total-Body Dose Commitment from Airborne Pathways

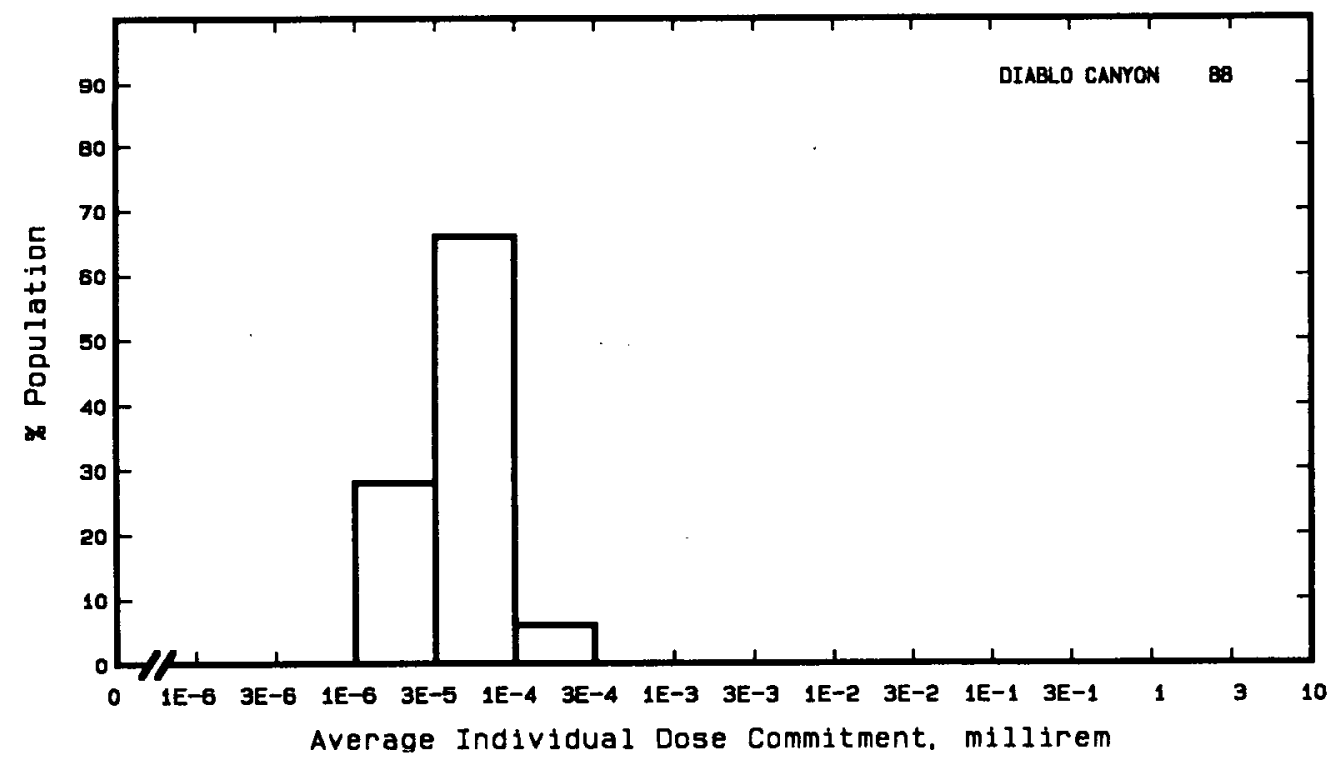


Site: DRESDEN

Location: $\quad$ N $41.3897^{\circ}$
GRUNDY COUNTY, ILLINOIS

W $88.2711^{\circ}$

POPULATION DATA

Total Population Within 2-to-80-km Region: 6.5E6

Major Metropolitan Centers Within Region:

Center

Chicago SMSA

Gary-Hammond-E.Chicago-SMSA

Kankakee SMSA

Aurora

Joliet

Elgin
Population

$7,200,000(a)$
$650,000(a)$
100,000
82,000
79,000
57,000

\section{Location}

$75 \mathrm{~km} \quad \mathrm{NE}$

$80 \mathrm{~km} \quad \mathrm{ENE}$

$45 \mathrm{~km} \quad \mathrm{SE}$

$41 \mathrm{~km} \mathrm{~N}$

$22 \mathrm{~km} \quad \mathrm{NE}$

$74 \mathrm{~km} \mathrm{~N}$

SITE-SPECIFIC DATA - AIRBORNE PATHWAYS

Average Annual State Production

of Crops and Animal Products

In 80-km Radius Circle

Regional Productivity Factor:

Animal Grazing Factor:
Veg: $1.1 \mathrm{E8}$ kilogram

Milk: 1.8E8 liter

Meat: $1.9 \mathrm{E} 8 \mathrm{kilogram}$

1

0.5

Meteorology Period of Record: 1 JAN 74 - 31 JAN 75 Recovery: $77 \%$

SITE-SPECIFIC DATA - WATERBORNE PATHWAYS via ILLINOIS RIVER

Average River Flow

at Site: $12,000 \mathrm{ft}^{3} / \mathrm{s}$

Drinking Water:

Exposed Population: (b)

Fish:

Edible Harvest: (b)

(a) Population of total SMSA given; population of SMSA fraction within $80 \mathrm{~km}$ of site would be somewhat smaller.

(b) River water used for sewage disposal for Chicago, so population doses from liquid pathways assumed to be near zero. 
POPULATION DOSE-COMMITMENT ESTIMATES AND

AVERAGE INDIVIDUAL DOSE-COMMITMENT HISTOGRAM FOR

DRESDEN 1, 2, AND 3

Dose Commitments (person-rem) from Waterborne Pathways

Iotal Body GI-LLI Ihyroid Bone Liver

Infant

Child

Teen

Adult

(Little or No Waterborne Pathway Doses)

TOTAL

Dose Commitments (person-rem) from Airborne Pathways

Total Body GI-LLI Thyroid Bone Liver Lung

$\begin{array}{lllllll}\text { Infant } & 1.4 \mathrm{E}-03 & 1.3 \mathrm{E}-03 & 7.5 \mathrm{E}-02 & 1.3 \mathrm{E}-03 & 1.6 \mathrm{E}-03 & 1.8 \mathrm{E}-03 \\ \text { Child } & 1.8 \mathrm{E}-02 & 1.7 \mathrm{E}-02 & 6.3 \mathrm{E}-01 & 1.4 \mathrm{E}-02 & 1.9 \mathrm{E}-02 & 2.4 \mathrm{E}-02 \\ \text { Teen } & 1.3 \mathrm{E}-02 & 1.3 \mathrm{E}-02 & 3.0 \mathrm{E}-01 & 9.4 \mathrm{E}-03 & 1.3 \mathrm{E}-02 & 1.9 \mathrm{E}-02 \\ \text { Adu1t } & 7.4 \mathrm{E}-02 & 7.8 \mathrm{E}-02 & 1.2 \mathrm{E}+00 & 5.4 \mathrm{E}-02 & 7.6 \mathrm{E}-02 & 1.0 \mathrm{E}-01 \\ \text { TOTAL } & 1.1 \mathrm{E}-01 & 1.1 \mathrm{E}-01 & 2.2 \mathrm{E}+00 & 7.9 \mathrm{E}-02 & 1.1 \mathrm{E}-01 & 1.4 \mathrm{E}-01\end{array}$

Production/Consumption factors:

Produce: 0.090 Mi7k: 0.21 Meat: 0.36

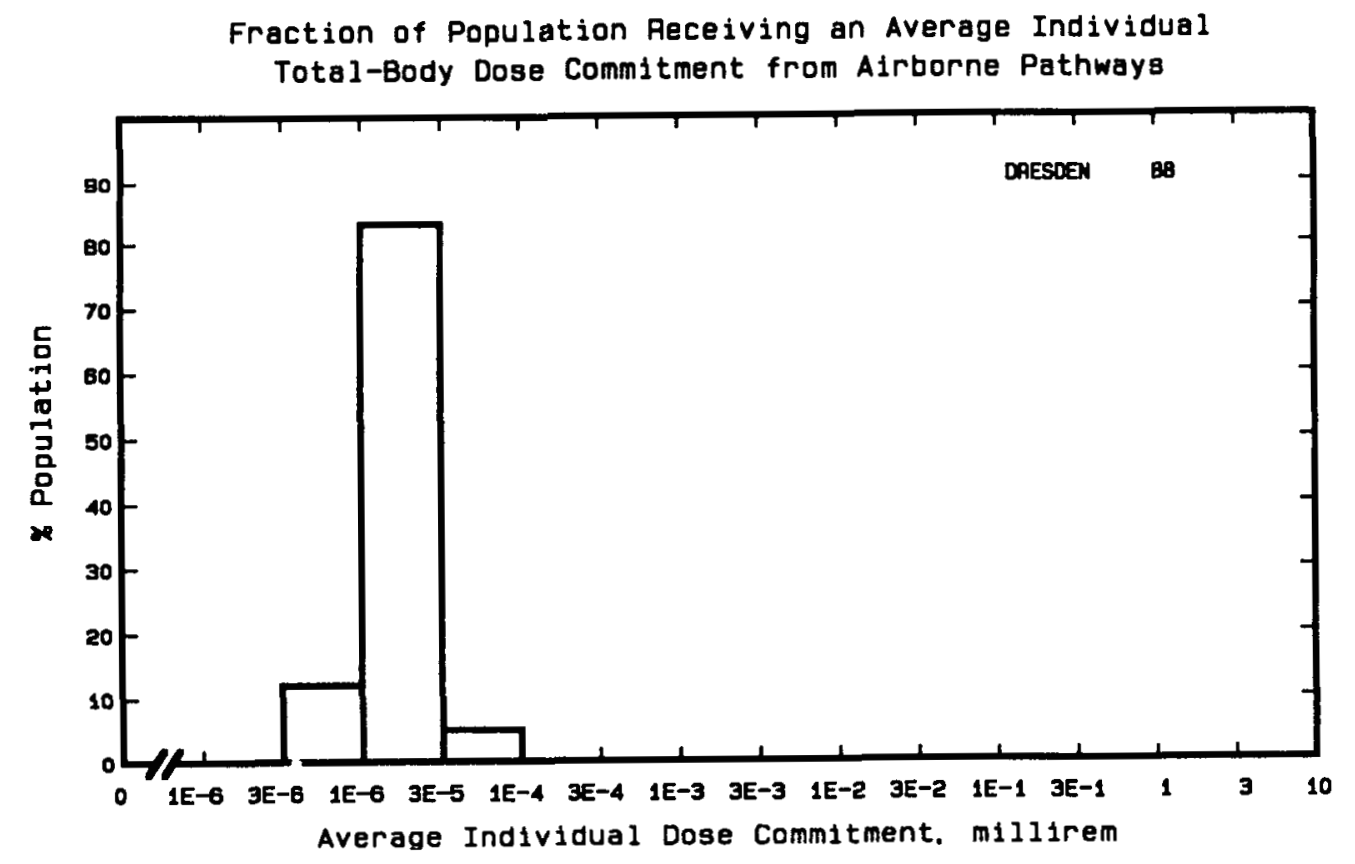


Site: DUANE ARNOLD

CEDAR RAPIDS, IOWA

Location: N $42.1006^{\circ} \quad$ W $91.7772^{\circ}$

POPULATION DATA

Total Population Within 2-to-80-km Region: 5.8E5

Major Metropolitan Centers Within Region:

Center Population Location

Cedar Rapids SMSA $\quad 170,000 \quad 17 \mathrm{~km} \quad$ SE

Waterloo-Cedar Falls SMSA $140,000 \quad 64 \mathrm{~km} \quad \mathrm{NW}$

Iowa City SMSA $\quad 81,000 \quad 52 \mathrm{~km}$ SSE

Marion $19,000 \quad 16 \mathrm{~km}$ ESE

SITE-SPECIFIC DATA - AIRBORNE PATHWAYS

Average Annual State Production

Veg: $9.8 \mathrm{E} 7 \mathrm{kilogram}$

of Crops and Animal Products

In 80-km Radius Circle

Milk: 2.6E8 liter

Meat: $4.2 \mathrm{E} 8 \mathrm{kilogram}$

Regional Productivity Factor:

Animal Grazing Factor:

1

0.5

Meteorology Period of Record: 1 FEB 71 - 31 DEC 75 Recovery: 57\%

SITE-SPECIFIC DATA - WATERBORNE PATHWAYS via CEDAR RIVER

Average River Flow

at Site: $3,100 \mathrm{ft}^{3} / \mathrm{s}$

Drinking Water: None

Fish: $\quad$ None 


\section{POPULATION DOSE-COMMITMENT ESTIMATES AND \\ AVERAGE INDIVIDUAL DOSE-COMMITMENT HISTOGRAM FOR \\ DUANE ARNOLD}

Dose Commitments (person-rem) from Waterborne Pathways

Total Body GI-LLI Thyroid Bone Liver

Infant

Child

Teen

Adult

(No Waterborne Pathway Doses) (a)

TOTAL

Dose Commitments (person-rem) from Airborne Pathways

Total Body GI-LLI Ihyroid Bone Liver Lung

$\begin{array}{lllllll}\text { Infant } & 8.9 \mathrm{E}-04 & 8.9 \mathrm{E}-04 & 3.1 \mathrm{E}-03 & 8.3 \mathrm{E}-04 & 9.0 \mathrm{E}-04 & 9.3 \mathrm{E}-04 \\ \text { Child } & 1.1 \mathrm{E}-02 & 1.1 \mathrm{E}-02 & 3.1 \mathrm{E}-02 & 9.3 \mathrm{E}-03 & 1.1 \mathrm{E}-02 & 1.1 \mathrm{E}-02 \\ \text { Teen } & 7.6 \mathrm{E}-03 & 7.7 \mathrm{E}-03 & 1.6 \mathrm{E}-02 & 6.7 \mathrm{E}-03 & 7.6 \mathrm{E}-03 & 8.3 \mathrm{E}-03 \\ \text { Adult } & 4.5 \mathrm{E}-02 & 4.6 \mathrm{E}-02 & 7.5 \mathrm{E}-02 & 4.1 \mathrm{E}-02 & 4.5 \mathrm{E}-02 & 4.8 \mathrm{E}-02 \\ \text { TOTAL } & 6.4 \mathrm{E}-02 & 6.5 \mathrm{E}-02 & 1.3 \mathrm{E}-01 & 5.7 \mathrm{E}-02 & 6.4 \mathrm{E}-02 & 6.9 \mathrm{E}-02\end{array}$

Production/Consumption factors:

Produce: 0.86

Milk: 3.3

Meat: 8.9

Fraction of Population Receiving an Average Individual

Total-Body Dose Commitment from Airborne Pathways

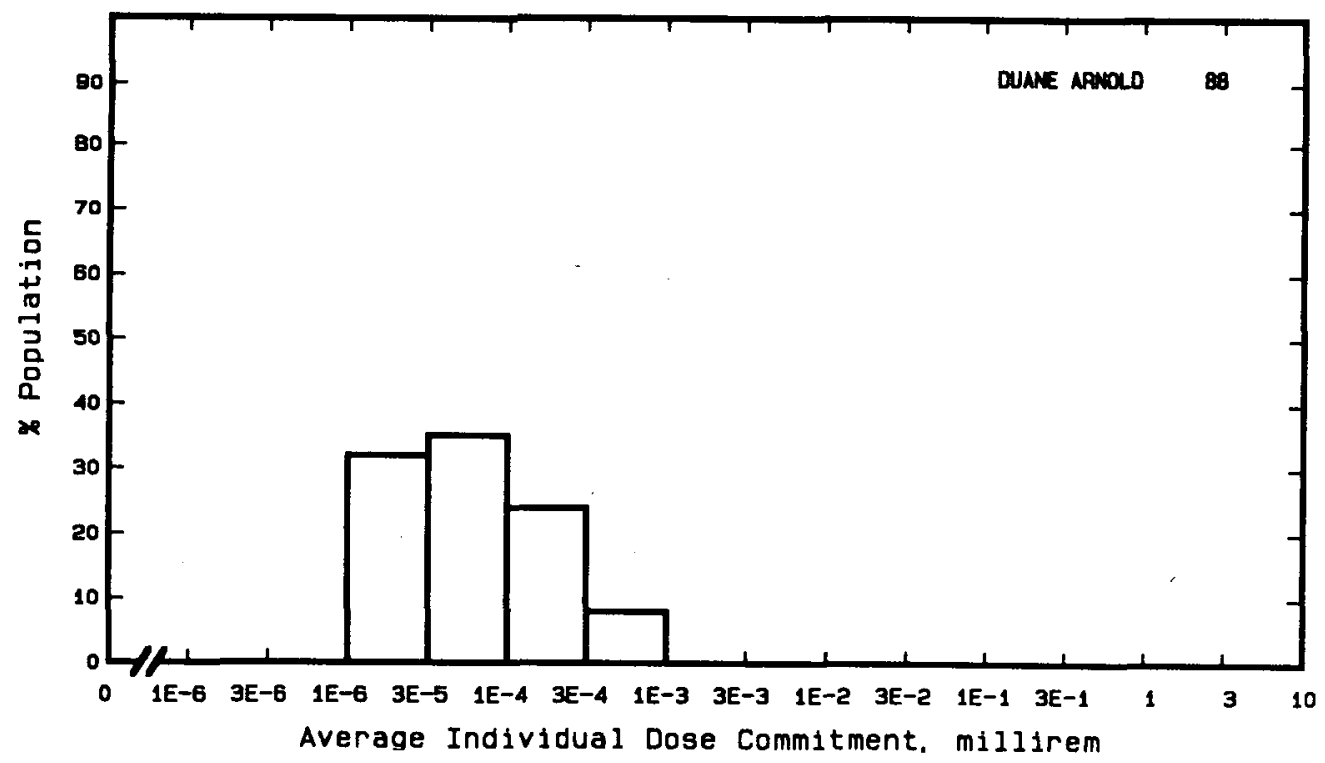

(a) No liquid releases reported (Tichler, et al. 1991). 
Site: J. M. FARLEY

DOTHAN, ALABAMA

Location: $\quad$ N $31.2228^{\circ} \quad$ W $85.1126^{\circ}$

POPULATION DATA

Total Population Within 2-to-80-km Region: 3.7E5

Major Metropolitan Centers Within Region:

Center

Dothan

Enterprise

Ozark

Eufaula

Bainbridge
Population

51,000

18,000

13,000

12,000

11,000
Location

$27 \mathrm{~km} \quad W$

$71 \mathrm{~km} \quad W$

$56 \mathrm{~km} \quad W N W$

$75 \mathrm{~km} \mathrm{~N}$

$62 \mathrm{~km} \quad \mathrm{SE}$

SITE-SPECIFIC DATA - AIRBORNE PATHWAYS

Average Annual State Production

of Crops and Animal Products

In $80-\mathrm{km}$ Radius Circle

Regional Productivity Factor:

Animal Grazing Factor:
Veg: 1.7E7 kilogram

Milk: 5.7E7 liter

Meat: $8.6 \mathrm{E} 7 \mathrm{kilogram}$

0.95

0.8

Meteorology Period of Record: 1 APR 71 - 31 MAR 75 Recovery: 100\%

Drinking Water:

Fish:
Average River Flow at Site: $12,000 \mathrm{ft}^{3} / \mathrm{s}$

None

Edible Harvest: $2.3 \mathrm{E} 5 \mathrm{~kg} / \mathrm{yr}$

Dilution Factor: 1 


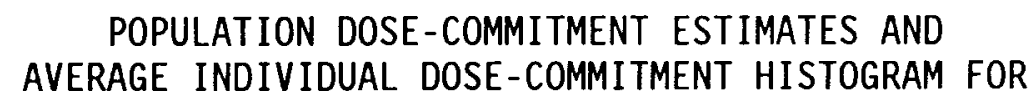

J. M. FARLEY 1 AND 2

Dose Commitments (person-rem) from Waterborne Pathways

Total Body GI-LLI Thyroid Bone Liver

\begin{tabular}{|c|c|c|c|c|c|c|c|}
\hline $\begin{array}{l}\text { Infant } \\
\text { Child } \\
\text { Teen } \\
\text { Adult }\end{array}$ & $\begin{array}{l}0.0 \mathrm{E}+00 \\
1.1 \mathrm{E}-03 \\
1.7 \mathrm{E}-03 \\
1.8 \mathrm{E}-02\end{array}$ & $\begin{array}{l}0.0 E+00 \\
3.5 E-03 \\
7.0 E-03 \\
6.0 E-02\end{array}$ & & $\begin{array}{l}0.0 \mathrm{E}+00 \\
3.2 \mathrm{E}-04 \\
2.9 \mathrm{E}-04 \\
2.3 \mathrm{E}-03\end{array}$ & & $\begin{array}{l}E+00 \\
E-03 \\
E-03 \\
E-02\end{array}$ & $\begin{array}{l}0.0 E+00 \\
4.8 E-03 \\
4.0 E-03 \\
2.4 E-02\end{array}$ \\
\hline \multirow[t]{3}{*}{ TOTAL } & $2.0 \mathrm{E}-02$ & $7.1 \mathrm{E}-02$ & & $2.9 E-03$ & & $E-02$ & $3.3 E-02$ \\
\hline & \multicolumn{7}{|c|}{ Dose Commitments (person-rem) from Airborne Pathways } \\
\hline & Total Body & $\underline{G I-L L I}$ & Thyroi & & & Liver & Lung \\
\hline $\begin{array}{l}\text { Infant } \\
\text { Child } \\
\text { Teen } \\
\text { Adult }\end{array}$ & $\begin{array}{l}5.9 E-04 \\
8.3 E-03 \\
5.4 E-03 \\
3.0 E-02\end{array}$ & $\begin{array}{l}5.9 E-04 \\
8.3 E-03 \\
5.4 E-03 \\
3.0 E-02\end{array}$ & $\begin{array}{l}9.4 E-0 \\
1.0 E-0 \\
6.2 E-0 \\
3.3 E-0\end{array}$ & & & $\begin{array}{l}5.9 E-04 \\
8.3 E-03 \\
5.4 E-03 \\
3.0 E-02\end{array}$ & $\begin{array}{l}6.2 E-04 \\
8.7 E-03 \\
5.9 E-03 \\
3.2 E-02\end{array}$ \\
\hline TOTAL & $4.5 E-02$ & $4.5 E-02$ & $5.1 \mathrm{E}-0$ & & & $4.5 \mathrm{E}-02$ & $4.7 E-02$ \\
\hline
\end{tabular}

Production/Consumption factors:

Produce: 0.22

Milk: 1.1

Meat: 2.7

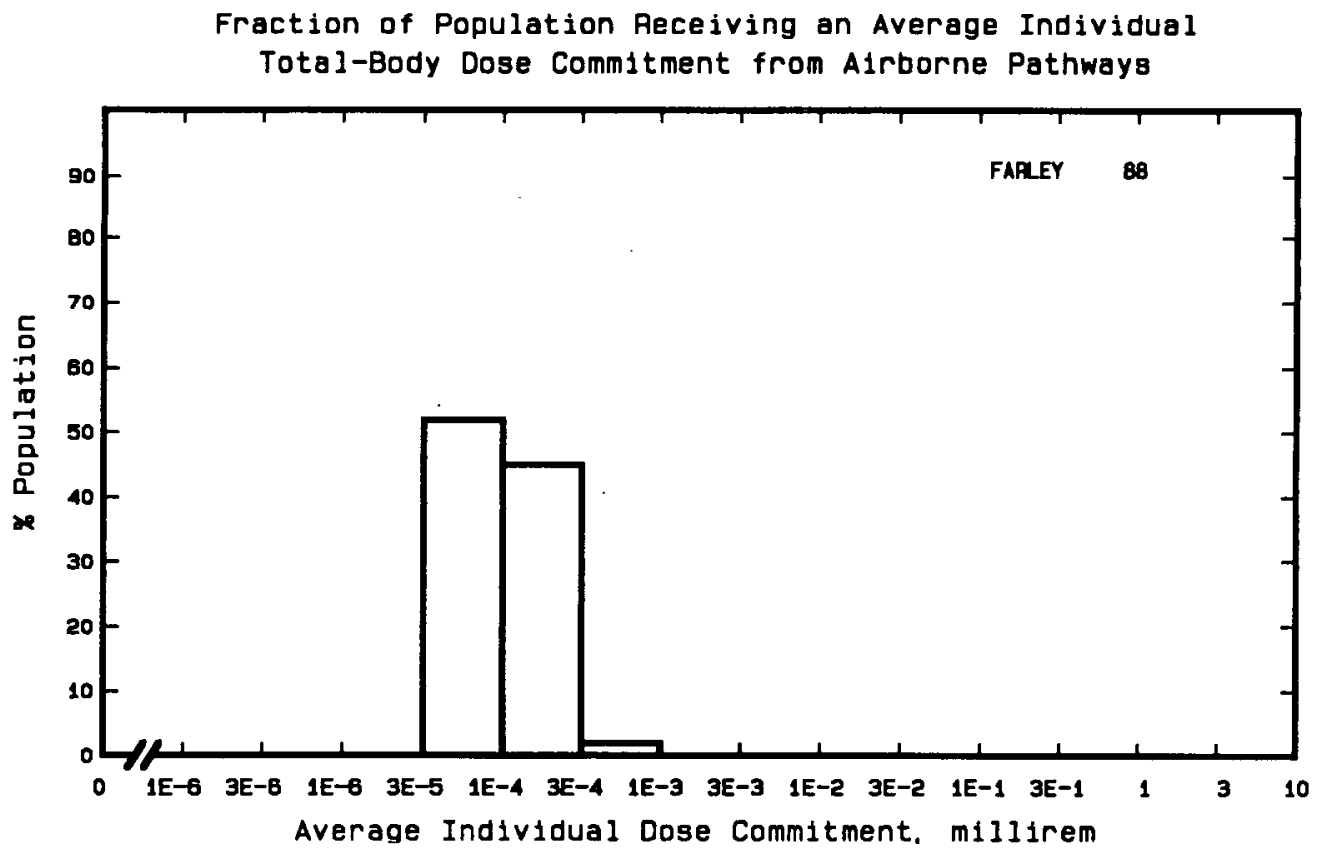


Site: FERMI

LAGOONA BEACH, MICHIGAN

Location: $\quad$ N $41.9781^{\circ} \quad$ W $83.2594^{\circ}$

POPULATION DATA

Total Population Within 2-to-80-km Region: $4.9 E 6$

Major Metropolitan Centers Within Region:

Center

Detroit

Toledo

Ann Arbor

Sandusky

Monroe
Population

$1,200,000$

350,000

110,000

31,000

26,000

\section{Location}

24-80 km NNW

$40 \mathrm{~km} \quad \mathrm{SW}$

$48 \mathrm{~km} \quad \mathrm{NW}$

$72 \mathrm{~km} \quad \mathrm{SE}$

$13 \mathrm{~km}$ WSW

SITE-SPECIFIC DATA - AIRBORNE PATHWAYS

Average Annual State Production

of Crops and Animal Products

In $80-\mathrm{km}$ Radius Circle

Regional Productivity Factor:

Animal Grazing Factor:
Veg: $6.8 \mathrm{E} 7 \mathrm{kilogram}$

Milk: 2.9E8 liter

Meat: $4.5 \mathrm{E} 7 \mathrm{kilogram}$

0.6

0.5

Meteorology Period of Record: 1 JUN 74 - 31 MAY 75 Recovery: 90\%

Drinking Water:

Fish:
Average Dilution Flow

from Plant: $44 \mathrm{ft}^{3} / \mathrm{s}$

Exposed Population: 520,000

Dilution Factor: 0.00056

Edible Harvest: $8.9 \mathrm{E} 6 \mathrm{~kg} / \mathrm{yr}$

Dilution Factor: 0.001 
POPULATION DOSE-COMMITMENT ESTIMATES AND

AVERAGE INDIVIDUAL DOSE-COMMITMENT HISTOGRAM FOR

FERMI 2

Dose Commitments (person-rem) from Waterborne Pathways

Total Body GI-LLI Ihyroid Bone Liver

$\begin{array}{llllll}\text { Infant } & 1.1 \mathrm{E}-05 & 2.0 \mathrm{E}-05 & 8.8 \mathrm{E}-06 & 1.7 \mathrm{E}-05 & 1.3 \mathrm{E}-05 \\ \text { Child } & 1.8 \mathrm{E}-03 & 2.4 \mathrm{E}-03 & 8.7 \mathrm{E}-05 & 1.3 \mathrm{E}-03 & 2.8 \mathrm{E}-03 \\ \text { Teen } & 1.2 \mathrm{E}-03 & 4.7 \mathrm{E}-03 & 3.1 \mathrm{E}-05 & 8.5 \mathrm{E}-04 & 2.6 \mathrm{E}-03 \\ \text { Adult } & 7.3 \mathrm{E}-03 & 4.2 \mathrm{E}-02 & 2.5 \mathrm{E}-04 & 5.7 \mathrm{E}-03 & 1.6 \mathrm{E}-02 \\ \text { TOTAL } & 1.0 \mathrm{E}-02 & 4.9 \mathrm{E}-02 & 3.8 \mathrm{E}-04 & 7.9 \mathrm{E}-03 & 2.1 \mathrm{E}-02\end{array}$

Dose Commitments (person-rem) from Airborne Pathways

Total Body GI-LLI Ihyroid Bone liver

$\begin{array}{lllllll}\text { Infant } & 2.9 \mathrm{E}-05 & 2.8 \mathrm{E}-05 & 7.7 \mathrm{E}-04 & 9.2 \mathrm{E}-05 & 3.0 \mathrm{E}-05 & 6.6 \mathrm{E}-05 \\ \text { Child } & 4.8 \mathrm{E}-04 & 3.3 \mathrm{E}-04 & 7.6 \mathrm{E}-03 & 2.3 \mathrm{E}-03 & 3.2 \mathrm{E}-04 & 9.1 \mathrm{E}-04 \\ \text { Teen } & 3.0 \mathrm{E}-04 & 3.0 \mathrm{E}-04 & 4.2 \mathrm{E}-03 & 1.6 \mathrm{E}-03 & 2.2 \mathrm{E}-04 & 7.4 \mathrm{E}-04 \\ \text { Adult } & 1.6 \mathrm{E}-03 & 1.7 \mathrm{E}-03 & 1.9 \mathrm{E}-02 & 8.3 \mathrm{E}-03 & 1.1 \mathrm{E}-03 & 3.1 \mathrm{E}-03 \\ \text { TOTAL } & 2.4 \mathrm{E}-03 & 2.3 \mathrm{E}-03 & 3.1 \mathrm{E}-02 & 1.2 \mathrm{E}-02 & 1.7 \mathrm{E}-03 & 4.9 \mathrm{E}-03\end{array}$

Production/Consumption factors:

Produce: $0.043 \quad$ Milk: 0.27 Meat: 0.068

Fraction of Population Receiving an Average Individual Total-Body Dose Commitment from Airborne Pathways

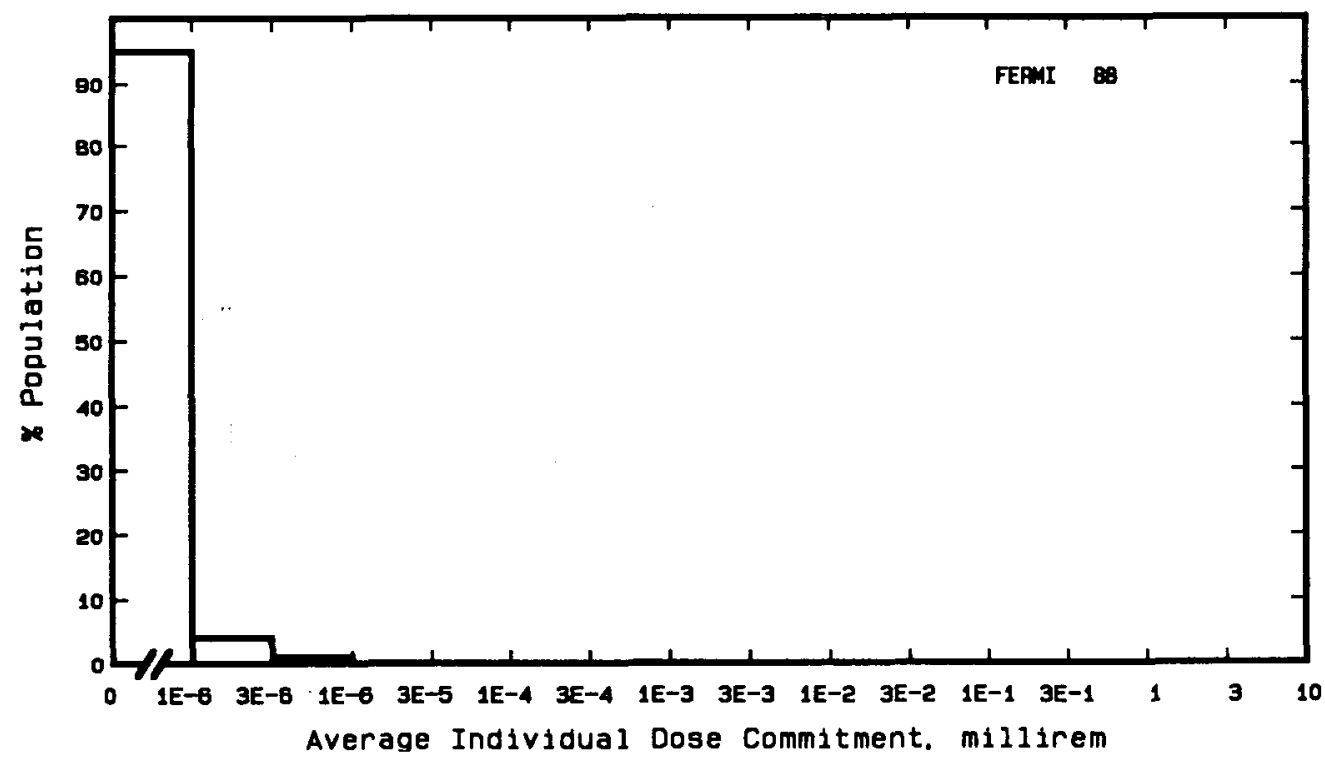


Site: J. A. FITZPATRICK

OSWEGO, NEW YORK

Location: $\quad$ N $43.5239^{\circ} \quad$ W $76.3983^{\circ}$

POPULATION DATA

Total Population Within 2-to-80-km Region: 8.6E5

Major Metropolitan Centers Within Region:

Center

Syracuse SMSA

Rome

Auburn

Watertown

Kingston
Population

650,000

48,000

33,000

28,000

24,000
Location

$56 \mathrm{~km} \quad$ SSE

$80 \mathrm{~km} \quad \mathrm{ESE}$

$66 \mathrm{~km} \quad \mathrm{SSW}$

$64 \mathrm{~km} \quad \mathrm{NE}$

$79 \mathrm{~km} \mathrm{~N}$

SITE-SPECIFIC DATA - AIRBORNE PATHWAYS

Average Annual State Production

of Crops and Animal Products

In 80-km Radius Circle

Regional Productivity Factor:

Animal Grazing Factor:
Veg: $7.6 \mathrm{E} 7 \mathrm{kilogram}$

Milk: 7.0E8 liter

Meat: $3.3 \mathrm{E} 7$ kilogram

Meteorology Period of Record: 1 JAN 74 - 31 DEC 75 Recovery: 97\%

Drinking Water:

Fish:
Average Dilution Flow

from Plant: $710 \mathrm{ft}^{3} / \mathrm{s}$

Exposed Population: 540,000 (a)

Dilution Factor: $0.003(b)$

Edible Harvest: $7.3 \mathrm{E} 5 \mathrm{~kg} / \mathrm{yr}$

Dilution Factor: $0.005(B)$

(a) Population exposed to drinking water derived from Nine Mile Point FES (1974).

(b) Dilution factors derived from FES (1973). 


\section{POPULATION DOSE-COMMITMENT ESTIMATES AND \\ AVERAGE INDIVIDUAL DOSE-COMMITMENT HISTOGRAM FOR}

J. A. FITZPATRICK

Dose Commitments (person-rem) from Waterborne Pathways

Total Body GI-LLI Ihyroid Bone Liver

$\begin{array}{llllll}\text { Infant } & 2.6 \mathrm{E}-05 & 2.1 \mathrm{E}-05 & 6.7 \mathrm{E}-05 & 4.8 \mathrm{E}-05 & 8.3 \mathrm{E}-05 \\ \text { Child } & 9.5 \mathrm{E}-04 & 3.1 \mathrm{E}-04 & 5.5 \mathrm{E}-04 & 3.8 \mathrm{E}-03 & 4.5 \mathrm{E}-03 \\ \text { Teen } & 1.4 \mathrm{E}-03 & 2.4 \mathrm{E}-04 & 1.9 \mathrm{E}-04 & 2.1 \mathrm{E}-03 & 3.4 \mathrm{E}-03 \\ \text { Adult } & 1.5 \mathrm{E}-02 & 2.1 \mathrm{E}-03 & 1.4 \mathrm{E}-03 & 1.2 \mathrm{E}-02 & 2.02 \mathrm{E}-02 \\ \text { TOTAL } & 1.7 \mathrm{E}-02 & 2.7 \mathrm{E}-03 & 2.2 \mathrm{E}-03 & 1.8 \mathrm{E}-02 & 2.8 \mathrm{E}-02\end{array}$

Dose Commitments (person-rem) from Airborne Pathways

Total Body GI-LLI Ihyroid Bone Liver Lung

$\begin{array}{lllllll}\text { Infant } & 2.1 \mathrm{E}-03 & 2.1 \mathrm{E}-03 & 1.3 \mathrm{E}-02 & 2.1 \mathrm{E}-03 & 2.1 \mathrm{E}-03 & 2.1 \mathrm{E}-03 \\ \text { Child } & 2.3 \mathrm{E}-02 & 2.3 \mathrm{E}-02 & 9.3 \mathrm{E}-02 & 2.3 \mathrm{E}-02 & 2.3 \mathrm{E}-02 & 2.4 \mathrm{E}-02 \\ \text { Teen } & 1.7 \mathrm{E}-02 & 1.7 \mathrm{E}-02 & 4.3 \mathrm{E}-02 & 1.7 \mathrm{E}-02 & 1.7 \mathrm{E}-02 & 1.8 \mathrm{E}-02 \\ \text { Adult } & 1.0 \mathrm{E}-01 & 1.0 \mathrm{E}-01 & 1.9 \mathrm{E}-01 & 1.0 \mathrm{E}-01 & 1.0 \mathrm{E}-01 & 1.1 \mathrm{E}-01 \\ \text { TOTAL } & 1.4 \mathrm{E}-01 & 1.4 \mathrm{E}-01 & 3.3 \mathrm{E}-01 & 1.4 \mathrm{E}-01 & 1.4 \mathrm{E}-01 & 1.5 \mathrm{E}-01\end{array}$

Production/Consumption factors:

Produce: $0.32 \quad$ Milk: $4.4 \quad$ Meat: 0.34

Fraction of Population Receiving an Average Individual

Total-Body Dose Commitment from Airborne Pathways

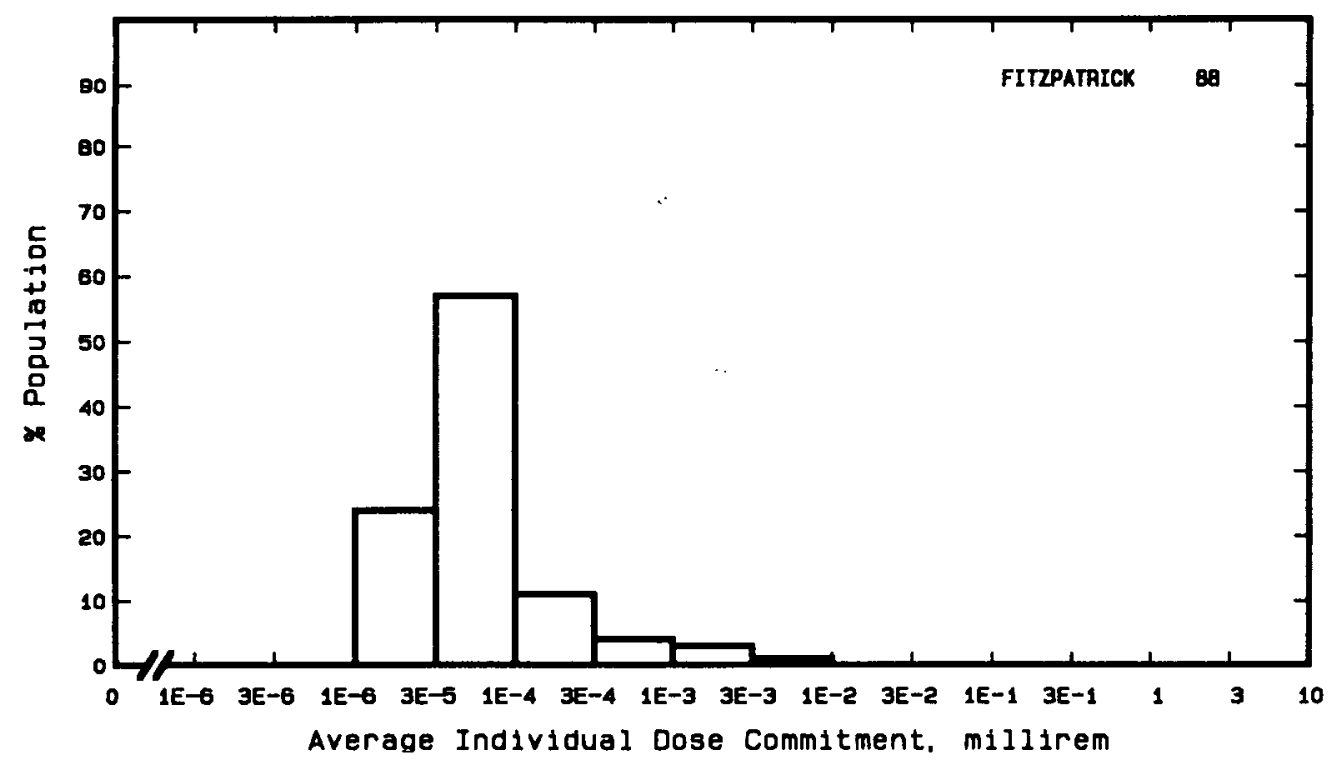


Site: FORT CALHOUN

WASHINGTON COUNTY, NEBRASKA

Location: $\quad N 41.5208^{\circ} \quad$ W $96.0767^{\circ}$

POPULATION DATA

Total Population Within 2-to-80-km Region: 7.6E5

Major Metropolitan Centers Within Region:

Center

Omaha SMSA

Council Bluffs

Freemont

Bellevue
Population

610,000

56,000

24,000

22,000
Location

$32 \mathrm{~km} \quad$ SSE

$34 \mathrm{~km} \quad \mathrm{SE}$

$36 \mathrm{~km}$ WSW

$44 \mathrm{~km} \quad$ SSE

SITE-SPECIFIC DATA - AIRBORNE PATHHAYS

Average Annual State Production

of Crops and Animal Products

In 80-km Radius Circle

Veg: $9.7 E 7$ kilogram

Milk: $7.2 E 7$ liter

Meat: $2.0 E 8$ kilogram

Regional Productivity Factor:

Animal Grazing Factor:

1

0.5

Meteorology Period of Record: 1 JAN 74 - 31 DEC 74 Recovery: 98\%

SITE-SPECIFIC DATA - WATERBORNE PATHWAYS via MISSOURI RIVER

Average River Flow

at Site: $27,000 \mathrm{ft}^{3} / \mathrm{s}$

Drinking Water:

Exposed Population: 580,000 (a)

Dilution Factor: 1

Fish:

Edible Harvest: $1.0 \mathrm{E} 4 \mathrm{~kg} / \mathrm{yr}$

Dilution Factor: 1

(a) Drinking water population assumed to be approximately Omaha SMSA (FES 1972). 


\section{POPULATION DOSE-COMMITMENT ESTIMATES AND \\ AVERAGE INDIVIDUAL DOSE-COMMITMENT HISTOGRAM FOR}

FORT CALHOUN

Dose Commitments (person-rem) from Waterborne Pathways

Total Body GI-LLI Ihyroid Bone Liver

$\begin{array}{llllll}\text { Infant } & 4.8 \mathrm{E}-03 & 4.4 \mathrm{E}-03 & 2.2 \mathrm{E}-02 & 4.9 \mathrm{E}-03 & 9.4 \mathrm{E}-03 \\ \text { Child } & 5.8 \mathrm{E}-02 & 5.0 \mathrm{E}-02 & 1.7 \mathrm{E}-01 & 5.5 \mathrm{E}-02 & 9.5 \mathrm{E}-02 \\ \text { Teen } & 2.6 \mathrm{E}-02 & 2.1 \mathrm{E}-02 & 5.5 \mathrm{E}-02 & 1.5 \mathrm{E}-02 & 3.6 \mathrm{E}-02 \\ \text { Adult } & 2.4 \mathrm{E}-01 & 1.8 \mathrm{E}-01 & 4.2 \mathrm{E}-01 & 9.9 \mathrm{E}-02 & 2.7 \mathrm{E}-01 \\ \text { TOTAL } & 3.3 \mathrm{E}-01 & 2.5 \mathrm{E}-01 & 6.6 \mathrm{E}-01 & 1.7 \mathrm{E}-01 & 4.1 \mathrm{E}-01\end{array}$

Dose Commitments (person-rem) from Airborne Pathways

Total Body GI-LLI Ihyroid Bone Liver Lung

$\begin{array}{lllllll}\text { Infant } & 1.1 \mathrm{E}-04 & 1.1 \mathrm{E}-04 & 2.4 \mathrm{E}-04 & 1.0 \mathrm{E}-04 & 1.1 \mathrm{E}-04 & 1.2 \mathrm{E}-04 \\ \text { Child } & 1.3 \mathrm{E}-03 & 1.3 \mathrm{E}-03 & 2.5 \mathrm{E}-03 & 1.2 \mathrm{E}-03 & 1.3 \mathrm{E}-03 & 1.4 \mathrm{E}-03 \\ \text { Teen } & 9.3 \mathrm{E}-04 & 9.3 \mathrm{E}-04 & 1.4 \mathrm{E}-03 & 8.4 \mathrm{E}-04 & 9.4 \mathrm{E}-04 & 1.1 \mathrm{E}-03 \\ \text { Adult } & 5.6 \mathrm{E}-03 & 5.6 \mathrm{E}-03 & 7.4 \mathrm{E}-03 & 5.1 \mathrm{E}-03 & 5.6 \mathrm{E}-03 & 6.2 \mathrm{E}-03 \\ \text { TOTAL } & 8.0 \mathrm{E}-03 & 7.9 \mathrm{E}-03 & 1.2 \mathrm{E}-02 & 7.2 \mathrm{E}-03 & 8.0 \mathrm{E}-03 & 8.9 \mathrm{E}-03\end{array}$

Production/Consumption factors:

Produce: $0.65 \quad$ Milk: $0.72 \quad$ Meat: 3.2

Fraction of Population Aeceiving an Average Individual

Total-Body Dose Commitment from Airborne Pathways

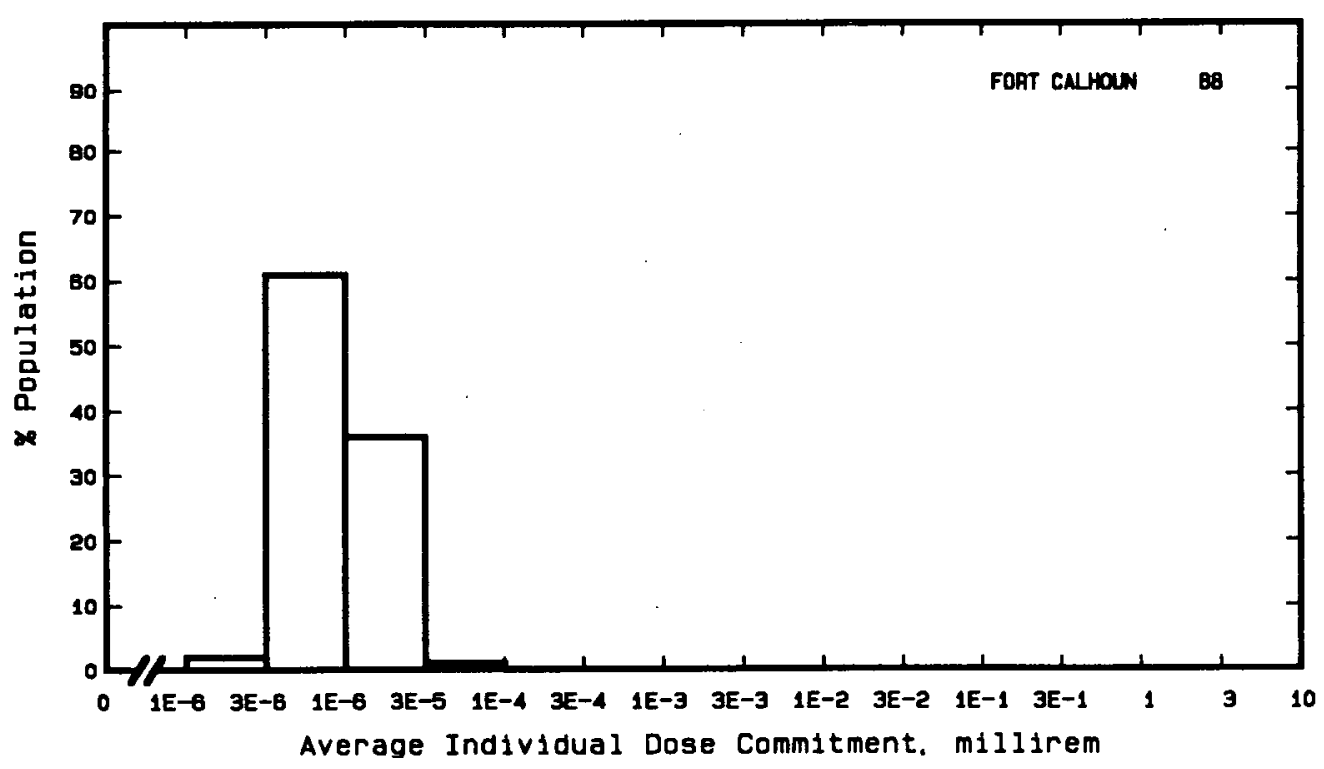


Site: R. E. GINNA

ONTARIO, NEW YORK

Location: $\quad$ N $43.2778^{\circ} \quad$ W $77.3089^{\circ}$

POPULATION DATA

Total Population Within 2-to-80-km Region: 1.2E6

Major Metropolitan Centers Within Region:

$\quad$ Center
Rochester SMSA
Auburn
Oswego
Batavia
Geneva

Population

Location

SITE-SPECIFIC DATA - AIRBORNE PATHWAYS

Average Annual State Production

of Crops and Animal Products

In 80-km Radius Circle

990,000

32,000

20,000

17,000

$27 \mathrm{~km} \quad$ WSW

$71 \mathrm{~km} \quad \mathrm{ESE}$

$67 \mathrm{~km} \quad$ ENE

$78 \mathrm{~km} \quad$ WSW

15,000

$52 \mathrm{~km} \quad$ SSE

Regional Productivity Factor:

Animal Grazing Factor:

Veg: $7.6 \mathrm{E} 7 \mathrm{kilogram}$

Milk: 7.0E8 liter

Meat: $3.3 \mathrm{E} 7$ kilogram

Meteorology Period of Record: 1 JAN 66 - 31 DEC 67 Recovery: 89\%

SITE-SPECIFIC DATA - WATERBORNE PATHWAYS via LAKE ONTARIO

Average Dilution Flow

from Plant: $690 \mathrm{ft}^{3} / \mathrm{s}$

Drinking Water:

Exposed Population: 570,000

Dilution Factor: $0.01(a)$

Fish:

Edible Harvest: $7.3 \mathrm{E} 5 \mathrm{~kg} / \mathrm{yr}$

Dilution Factor: $0.01(\mathrm{a})$

(a) Dilution factors from FES (1973). 
POPULATION DOSE-COMMITMENT ESTIMATES AND

AVERAGE INDIVIDUAL DOSE-COMMITMENT HISTOGRAM FOR

$$
\text { R. E. GINNA }
$$

Dose Commitments (person-rem) from Waterborne Pathways

$$
\text { Total Body GI-LLI Ihyroid Bone Liver }
$$

$\begin{array}{llllll}\text { Infant } & 2.5 \mathrm{E}-03 & 2.4 \mathrm{E}-03 & 5.5 \mathrm{E}-03 & 1.6 \mathrm{E}-04 & 2.6 \mathrm{E}-03 \\ \text { Child } & 2.9 \mathrm{E}-02 & 2.7 \mathrm{E}-02 & 4.9 \mathrm{E}-02 & 6.7 \mathrm{E}-03 & 3.4 \mathrm{E}-02 \\ \text { Teen } & 1.3 \mathrm{E}-02 & 1.1 \mathrm{E}-02 & 1.7 \mathrm{E}-02 & 3.5 \mathrm{E}-03 & 1.6 \mathrm{E}-02 \\ \text { Adult } & 1.1 \mathrm{E}-01 & 9.0 \mathrm{E}-02 & 1.4 \mathrm{E}-01 & 2.0 \mathrm{E}-02 & 1.2 \mathrm{E}-01 \\ \text { TOTAL } & 1.6 \mathrm{E}-01 & 1.3 \mathrm{E}-01 & 2.1 \mathrm{E}-01 & 3.1 \mathrm{E}-02 & 1.7 \mathrm{E}-01\end{array}$

Dose Commitments (person-rem) from Airborne Pathways

Total Body GI-LLI. Thyroid Bone Liver Lung

$\begin{array}{lllllll}\text { Infant } & 2.1 \mathrm{E}-03 & 2.1 \mathrm{E}-03 & 2.2 \mathrm{E}-03 & 6.8 \mathrm{E}-03 & 2.1 \mathrm{E}-03 & 2.1 \mathrm{E}-03 \\ \text { Child } & 2.1 \mathrm{E}-02 & 2.1 \mathrm{E}-02 & 2.1 \mathrm{E}-02 & 5.8 \mathrm{E}-02 & 2.1 \mathrm{E}-02 & 2.1 \mathrm{E}-02 \\ \text { Teen } & 9.2 \mathrm{E}-03 & 9.2 \mathrm{E}-03 & 9.4 \mathrm{E}-03 & 1.8 \mathrm{E}-02 & 9.2 \mathrm{E}-03 & 9.2 \mathrm{E}-03 \\ \text { Adult } & 4.0 \mathrm{E}-02 & 4.0 \mathrm{E}-02 & 4.1 \mathrm{E}-02 & 5.5 \mathrm{E}-02 & 4.0 \mathrm{E}-02 & 4.0 \mathrm{E}-02 \\ \text { TOTAL } & 7.3 \mathrm{E}-02 & 7.3 \mathrm{E}-02 & 7.4 \mathrm{E}-02 & 1.4 \mathrm{E}-01 & 7.3 \mathrm{E}-02 & 7.3 \mathrm{E}-02\end{array}$

Production/Consumption factors:

Produce: 0.19 Milk: $2.6 \quad$ Meat: 0.20

Fraction of Population Aeceiving an Average Individual Total-Body Dose Commitment from Alrborne Pathways

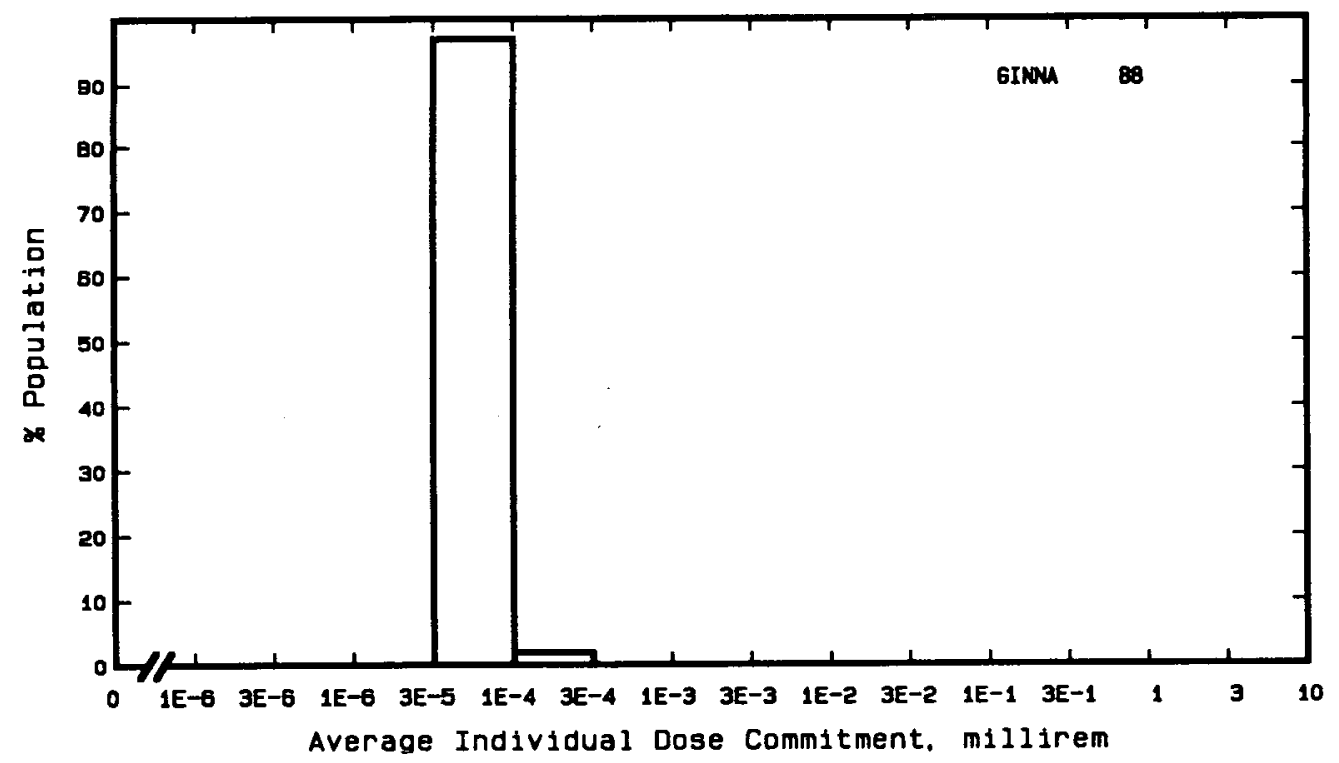


Site: GRAND GULF

PORT GIBSON, MISSISSIPPI

Location: $\quad N \quad 32.0270^{\circ} \quad$ W $91.2530^{\circ}$

POPULATION DATA

Total Population Within 2-to-80-km Region: 3.4E5

Major Metropolitan Centers Within Region:

Center

Vicksburg

Tallulah

Natches

Brookhaven
Population

27,000

10,000

20,000

11,000
Location

$40 \mathrm{~km} \quad$ NNE

$45 \mathrm{~km} \quad \mathrm{NNW}$

$60 \mathrm{~km} \quad \mathrm{SSW}$

$76 \mathrm{~km} \quad \mathrm{SE}$

SITE-SPECIFIC DATA - AIRBORNE PATHWAYS

Average Annual State Production

of Crops and Animal Products

In 80-km Radius Circle

Regional Productivity Factor:

Animal Grazing Factor:
Veg: 4.4E6 kilogram

Milk: $7.1 E 7$ liter

Meat: 9.9E7 kilogram

0.9

0.8

Meteorology Period of Record: 1 AUG 72 - 31 JUL 73 Recovery: 99\%

SITE-SPECIFIC DATA - WATERBORNE PATHWAYS via MISSISSIPPI RIVER

Average River

Flow at Site: $430,000 \mathrm{ft}^{3} / \mathrm{s}$

Drinking Water:

None

Fish:

Edible Harvest: $7.0 \mathrm{E} 5 \mathrm{~kg} / \mathrm{yr}$

Dilution Factor: 1

Invertebrates:

Edible Harvest: $7.0 \mathrm{E} 3 \mathrm{~kg} / \mathrm{yr}$

Dilution Factor: 1 
POPULATION DOSE-COMMITMENT ESTIMATES AND

AVERAGE INDIVIDUAL DOSE-COMMITMENT HISTOGRAM FOR

GRAND GULF

Dose Commitments (person-rem) from Waterborne Pathways

Total Body GI-LLI $\underline{\text { Thyroid Bone }}$

$\begin{array}{llllll}\text { Infant } & 0.0 \mathrm{E}+00 & 0.0 \mathrm{E}+00 & 0.0 \mathrm{E}+00 & 0.0 \mathrm{E}+00 & 0.0 \mathrm{E}+00 \\ \text { Child } & 2.6 \mathrm{E}-05 & 5.4 \mathrm{E}-05 & 3.9 \mathrm{E}-07 & 5.3 \mathrm{E}-05 & 1.1 \mathrm{E}-04 \\ \text { Teen } & 3.2 \mathrm{E}-05 & 1.2 \mathrm{E}-04 & 3.3 \mathrm{E}-07 & 3.2 \mathrm{E}-05 & 9.4 \mathrm{E}-05 \\ \text { Adult } & 2.9 \mathrm{E}-04 & 1.1 \mathrm{E}-03 & 2.5 \mathrm{E}-06 & 1.9 \mathrm{E}-04 & 5.7 \mathrm{E}-04 \\ \text { TOTAL } & 3.5 \mathrm{E}-04 & 1.3 \mathrm{E}-03 & 3.3 \mathrm{E}-06 & 2.7 \mathrm{E}-04 & 7.7 \mathrm{E}-04\end{array}$

Dose Commitments (person-rem) from Airborne Pathways

Total Body GI-LLI Thyroid Bone Liver lung

$\begin{array}{lllllll}\text { Infant } & 1.0 \mathrm{E}-05 & 1.0 \mathrm{E}-05 & 2.9 \mathrm{E}-05 & 3.7 \mathrm{E}-06 & 1.0 \mathrm{E}-05 & 1.1 \mathrm{E}-05 \\ \text { Child } & 1.3 \mathrm{E}-04 & 1.4 \mathrm{E}-04 & 2.3 \mathrm{E}-04 & 4.2 \mathrm{E}-05 & 1.3 \mathrm{E}-04 & 1.3 \mathrm{E}-04 \\ \text { Teen } & 8.5 \mathrm{E}-05 & 9.8 \mathrm{E}-05 & 1.3 \mathrm{E}-04 & 3.0 \mathrm{E}-05 & 8.5 \mathrm{E}-05 & 9.1 \mathrm{E}-05 \\ \text { Adult } & 4.9 \mathrm{E}-04 & 6.1 \mathrm{E}-04 & 6.3 \mathrm{E}-04 & 1.8 \mathrm{E}-04 & 4.9 \mathrm{E}-04 & 5.1 \mathrm{E}-04 \\ \text { TOTAL } & 7.1 \mathrm{E}-04 & 8.6 \mathrm{E}-04 & 1.0 \mathrm{E}-03 & 2.6 \mathrm{E}-04 & 7.1 \mathrm{E}-04 & 7.5 \mathrm{E}-04\end{array}$

Production/Consumption factors:

Produce: $0.060 \quad$ Milk: $1.5 \quad$ Meat: 3.3

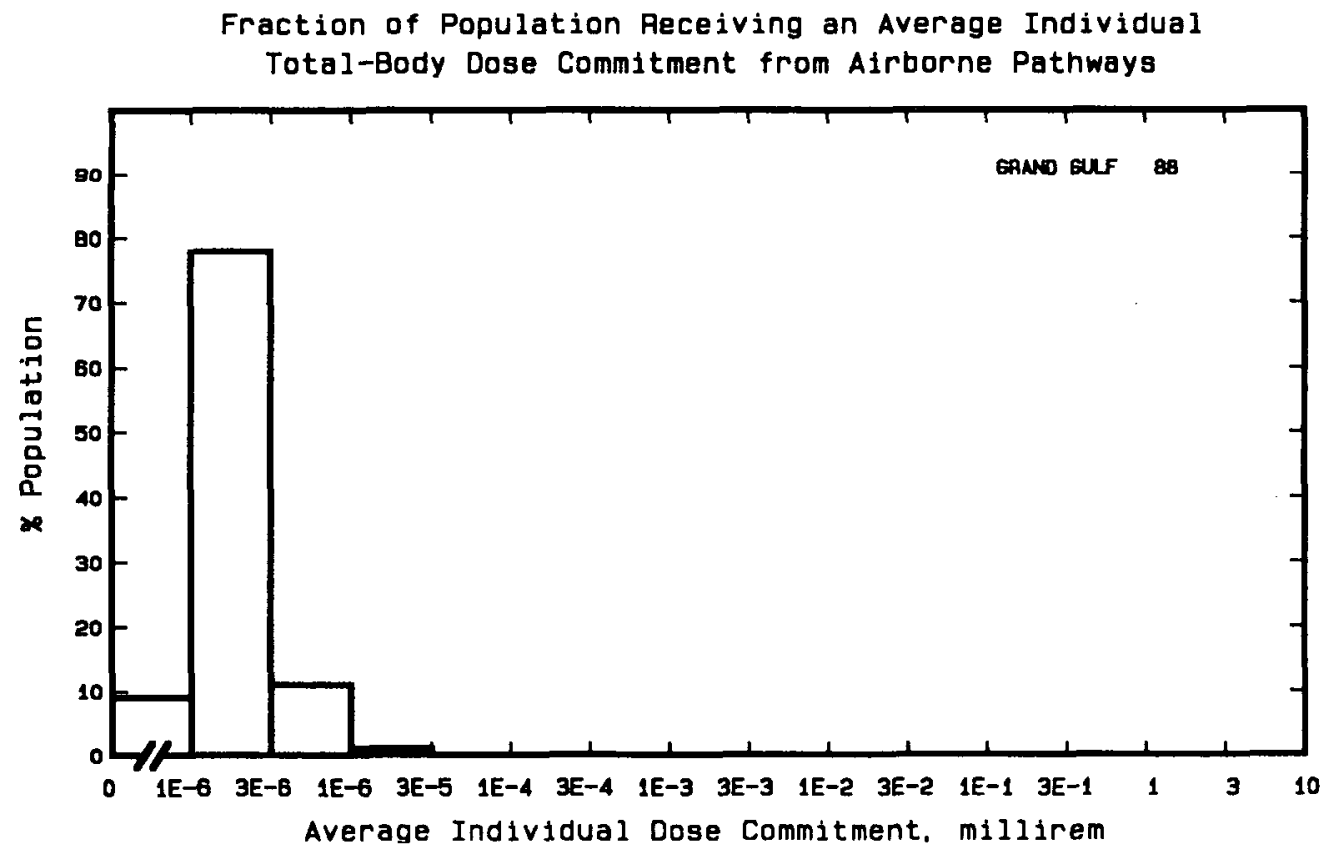


Site: HADDAM NECK

HADDAM NECK, CONNECTICUT

Location: N $\quad$ N1.4819 $\quad$ W $72.4992^{\circ}$

POPULATION DATA

Total Population Within 2-to-80-km Region: 3.5E6

Major Metropolitan Centers Within Region:

Center

Hartford SMSA

Springfield-Chicopee-Holyoke SMSA

New Haven-West Haven SMSA

Bridgeport SMSA

New London-Norwich
Population

750,000

550,000

440,000

420,000

250,000
Location

$35 \mathrm{~km} \quad \mathrm{NNW}$

$70 \mathrm{~km} \mathrm{~N}$

$40 \mathrm{~km} \quad$ WSW

$66 \mathrm{~km} \quad$ WSW

$35 \mathrm{~km} \quad$ ESE

SITE-SPECIFIC DATA - AIRBORNE PATHWAYS

Average Annual State Production

of Crops and Animal Products

In $80-\mathrm{km}$ Radius Circle

Veg: $3.2 E 7 \mathrm{kilogram}$

Milk: 4.4E8 liter

Meat: 2.0E7 kilogram

Regional Productivity Factor:

Animal Grazing Factor:

0.7

0.6

Meteorology Period of Record: 1 JAN 75 - 31 DEC 75 Recovery: 95\%

SITE-SPECIFIC DATA - WATERBORNE PATHWAYS via DISCHARGE CANAL TO CONN

RIVER

Average Discharge Canal

Flow at Site: $690 \mathrm{ft}^{3} / \mathrm{s}$

Drinking Water:

None

Fish:

Edible Harvest: $9.1 \mathrm{E} 3^{(\mathrm{a})} \mathrm{kg} / \mathrm{yr}$

Dilution Factor: 1

(a) Caught in discharge canal according to FES (1973). 
POPULATION DOSE-COMMITMENT ESTIMATES AND

AVERAGE INDIVIDUAL DOSE-COMMITMENT HISTOGRAM FOR

HADDAM NECK

Dose Commitments (person-rem) from Waterborne Pathways

Total Body GI-LLI Ihyroid Bone Liver

$\begin{array}{llllll}\text { Infant } & 0.0 \mathrm{E}+00 & 0.0 \mathrm{E}+00 & 0.0 \mathrm{E}+00 & 0.0 \mathrm{E}+00 & 0.0 \mathrm{E}+00 \\ \text { Child } & 3.5 \mathrm{E}-03 & 4.8 \mathrm{E}-04 & 1.8 \mathrm{E}-03 & 1.8 \mathrm{E}-02 & 1.9 \mathrm{E}-02 \\ \text { Teen } & 6.3 \mathrm{E}-03 & 7.6 \mathrm{E}-04 & 1.3 \mathrm{E}-03 & 1.1 \mathrm{E}-02 & 1.6 \mathrm{E}-02 \\ \text { Adult } & 6.8 \mathrm{E}-02 & 6.4 \mathrm{E}-03 & 8.8 \mathrm{E}-03 & 6.3 \mathrm{E}-02 & 9.4 \mathrm{E}-02 \\ \text { TOTAL } & 7.7 \mathrm{E}-02 & 7.6 \mathrm{E}-03 & 1.2 \mathrm{E}-02 & 9.1 \mathrm{E}-02 & 1.3 \mathrm{E}-01\end{array}$

Dose Commitments (person-rem) from Airborne Pathways

Total Body GI-LLI Thyroid Bone Liver Lung

$\begin{array}{lllllll}\text { Infant } & 6.5 \mathrm{E}-03 & 6.5 \mathrm{E}-03 & 7.2 \mathrm{E}-02 & 4.4 \mathrm{E}-03 & 6.7 \mathrm{E}-03 & 6.9 \mathrm{E}-03 \\ \text { Child } & 7.7 \mathrm{E}-02 & 7.6 \mathrm{E}-02 & 4.5 \mathrm{E}-01 & 4.9 \mathrm{E}-02 & 7.7 \mathrm{E}-02 & 8.2 \mathrm{E}-02 \\ \text { Teen } & 5.5 \mathrm{E}-02 & 5.4 \mathrm{E}-02 & 2.2 \mathrm{E}-01 & 3.5 \mathrm{E}-02 & 5.5 \mathrm{E}-02 & 6.2 \mathrm{E}-02 \\ \text { Adult } & 3.2 \mathrm{E}-01 & 3.2 \mathrm{E}-01 & 9.2 \mathrm{E}-01 & 2.1 \mathrm{E}-01 & 3.2 \mathrm{E}-01 & 3.4 \mathrm{E}-01 \\ \text { TOTAL } & 4.6 \mathrm{E}-01 & 4.5 \mathrm{E}-01 & 1.7 \mathrm{E}+00 & 3.0 \mathrm{E}-01 & 4.6 \mathrm{E}-01 & 4.9 \mathrm{E}-01\end{array}$

Production/Consumption factors:

Produce: 0.033 Milk: 0.67 Meat: 0.051

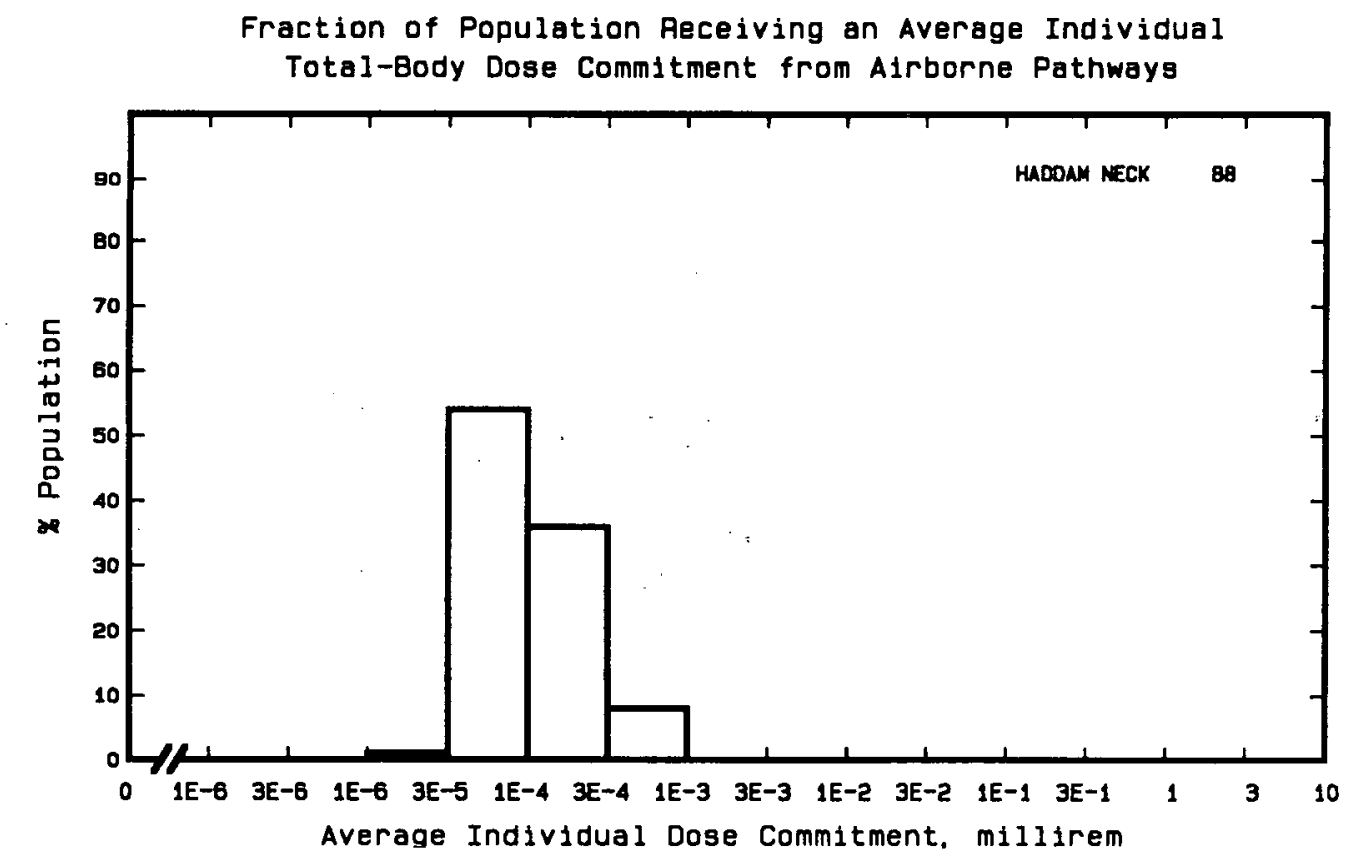


Site: HARRIS

NEWHILL, NORTH CAROLINA

Location: $\quad \mathrm{N} 35.6^{\circ} \quad$ W $79.0^{\circ}$

POPULATION DATA

Total Population Within 2-to-80-km Region: 1.4 E6

Major Metropolitan Centers Within Region:

Center Population Location

Raleigh-Durham SMSA $\quad 620,000 \quad 32 \mathrm{~km} \quad$ NE

Fayetteville $\quad 61,000 \quad 60 \mathrm{~km} \quad$ SSE

Burlington $\quad 38,000 \quad 64 \mathrm{~km}$ NW

Chapel Hill $\quad 34,000 \quad 32 \mathrm{~km}$ NNW

$\begin{array}{lll}\text { Sanford } & 16,000 & 22 \mathrm{~km} \quad \mathrm{SW}\end{array}$

SITE-SPECIFIC DATA - AIRBORNE PATHWAYS

Average Annual State Production

of Crops and Animal Products

In $80-\mathrm{km}$ Radius Circle

Regional Productivity Factor:

Animal Grazing Factor:
Veg: $2.6 \mathrm{E7}$ kilogram

Milk: 1.0E8 Titer

Meat: $5.8 \mathrm{E} 7$ kilogram

0.9

0.72

Meteorology Period of Record: 17 JAN 76 - 31 DEC 78 Recovery: 98\%

SITE-SPECIFIC DATA - WATERBORNE PATHWAYS via CAPE FEAR RIVER

Average River Flow

at Site: $3,200 \mathrm{ft}^{3} / \mathrm{s}$

Drinking Water: $\quad$ Exposed Population: 180,000

Dilution Factor: 1.18

Fish:

Edible Harvest: $0.024(\mathrm{a}) \mathrm{kg} / \mathrm{yr}$

Dilution Factor: $488(b)$

(a) Average individual consumption rate of $2.4 \mathrm{~kg} / \mathrm{yr}$ given in FES (1974) used in lieu of catch data.

(b) One percent of population is assumed to obtain fish taken from undiluted effluent. 


\section{POPULATION DOSE-COMMITMENT ESTIMATES AND}

AVERAGE INDIVIDUAL DOSE-COMMITMENT HISTOGRAM FOR

\section{HARRIS}

Dose Commitments (person-rem) from Waterborne Pathways

\begin{tabular}{|c|c|c|c|c|c|}
\hline & Total Body & GI-LLI & Thyroid & Bone & $\underline{\text { Liver }}$ \\
\hline $\begin{array}{l}\text { Infant } \\
\text { Child } \\
\text { Teen } \\
\text { Adult }\end{array}$ & $\begin{array}{l}2.3 E-02 \\
3.0 E-01 \\
1.4 E-01 \\
1.2 E+00\end{array}$ & $\begin{array}{l}2.3 \mathrm{E}-02 \\
3.6 \mathrm{E}-01 \\
2.9 \mathrm{E}-01 \\
2.5 \mathrm{E}+00\end{array}$ & $\begin{array}{l}2.4 \mathrm{E}-02 \\
2.9 \mathrm{E}-01 \\
1.3 \mathrm{E}-01 \\
1.1 \mathrm{E}+00\end{array}$ & $\begin{array}{l}4.4 E-05 \\
4.9 E-02 \\
2.9 E-02 \\
1.7 E-01\end{array}$ & $\begin{array}{l}2.3 \mathrm{E}-02 \\
3.4 \mathrm{E}-01 \\
1.7 \mathrm{E}-01 \\
1.3 \mathrm{E}+00\end{array}$ \\
\hline TOTAL & $1.7 E+00$ & $3.2 \mathrm{E}+00$ & $1.5 \mathrm{E}+00$ & $2.4 \mathrm{E}-01$ & $1.8 \mathrm{E}+00$ \\
\hline
\end{tabular}

Dose Commitments (person-rem) from Airborne Pathways

Total Body GI-LLI Thyroid Bone Liver Lung

$\begin{array}{lllllll}\text { Infant } & 1.5 \mathrm{E}-03 & 1.5 \mathrm{E}-03 & 1.5 \mathrm{E}-03 & 1.5 \mathrm{E}-03 & 1.5 \mathrm{E}-03 & 1.6 \mathrm{E}-03 \\ \text { Child } & 1.6 \mathrm{E}-02 & 1.6 \mathrm{E}-02 & 1.6 \mathrm{E}-02 & 1.6 \mathrm{E}-02 & 1.6 \mathrm{E}-02 & 1.8 \mathrm{E}-02 \\ \text { Teen } & 1.2 \mathrm{E}-02 & 1.2 \mathrm{E}-02 & 1.2 \mathrm{E}-02 & 1.2 \mathrm{E}-02 & 1.2 \mathrm{E}-02 & 1.4 \mathrm{E}-02 \\ \text { Adult } & 7.3 \mathrm{E}-02 & 7.3 \mathrm{E}-02 & 7.3 \mathrm{E}-02 & 7.3 \mathrm{E}-02 & 7.3 \mathrm{E}-02 & 8.0 \mathrm{E}-02 \\ \text { TOTAL } & 1.0 \mathrm{E}-01 & 1.0 \mathrm{E}-01 & 1.0 \mathrm{E}-01 & 1.0 \mathrm{E}-01 & 1.0 \mathrm{E}-01 & 1.1 \mathrm{E}-01\end{array}$

Production/Consumption factors:

Produce: $0.085 \quad$ Milk: $0.51 \quad$ Meat: 0.47

Fraction of Population Receiving an Average Individual

Total-Body Dose Commitment from Airborne Pathways

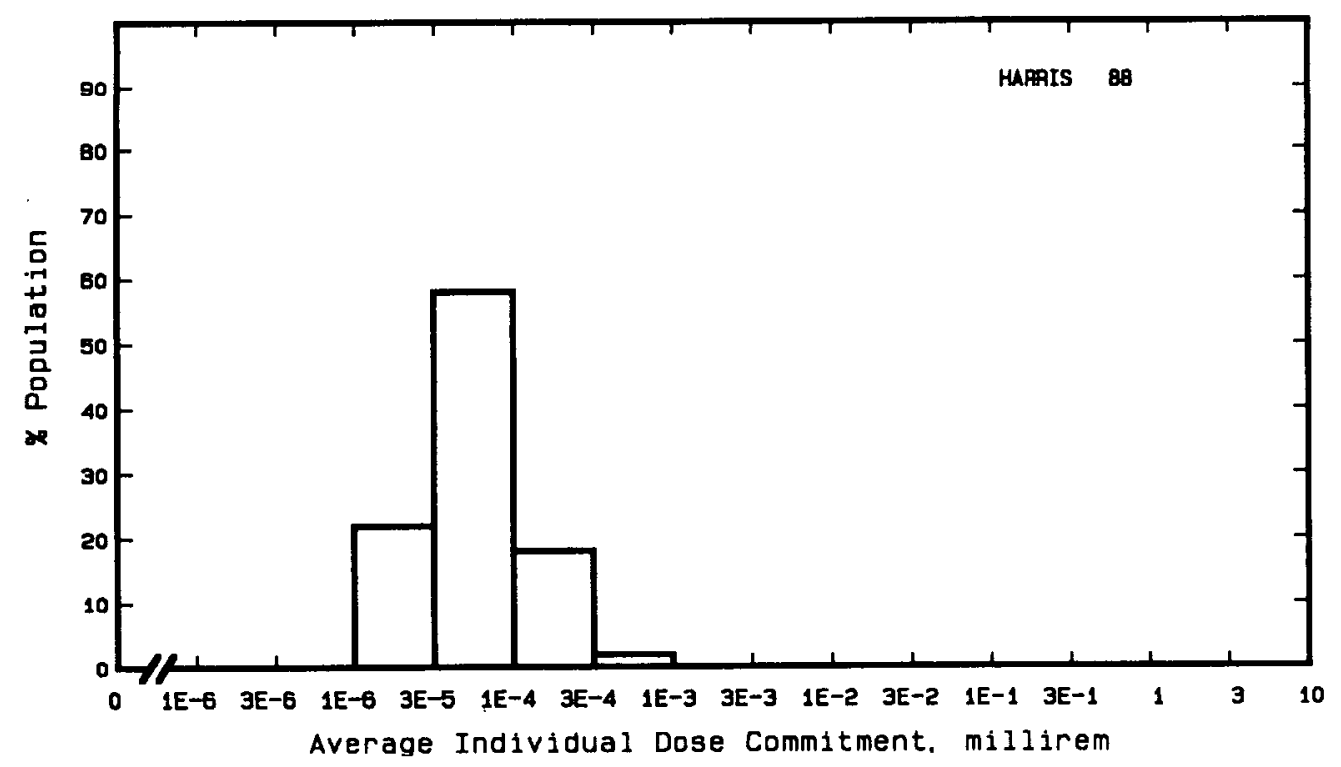


Site: E. I. HATCH

BAXLEY, GEORGIA

Location: $\quad$ N $31.9342^{\circ} \quad$ W $82.3444^{\circ}$

POPULATION DATA

Total Population Within 2-to-80-km Region: 3.5E5

Major Metropolitan Centers Within Region:

Center

Waycross

Statesboro

Hinesville

Douglas

Vidalia
Population

23,000

17,000

13,000

13,000

11,000
Location

$80 \mathrm{~km} \mathrm{~S}$

$78 \mathrm{~km} \quad \mathrm{NE}$

$71 \mathrm{~km} \quad \mathrm{E}$

$67 \mathrm{~km} \quad \mathrm{SW}$

$32 \mathrm{~km} \mathrm{~N}$

SITE-SPECIFIC DATA - AIRBORNE PATHWAYS

Average Annual State Production

of Crops and Animal Products

In 80-km Radius Circle

Regional Productivity Factor:

Animal Grazing Factor:
Veg: $8.8 \mathrm{E} 6 \mathrm{kilogram}$

Milk: 7.0E7 liter

Meat: 8.1E7 kilogram

1

0.8

Meteorology Period of Record: 1 JUN 70 - 31 AUG 74 Recovery: 87\%

\section{SITE-SPECIFIC DATA - WATERBORNE PATHWAYS via ALTAMAHA RIVER}

\section{Average River Flow}

at Site: $13,000 \mathrm{ft}^{3} / \mathrm{s}$

Drinking Water: None

Fish:

Edible Harvest: $6.3 \mathrm{E5}(\mathrm{a}) \mathrm{kg} / \mathrm{yr}$ Dilution Factor: 1

(a) Commercial catch plus 3 pounds of game fish per year taken from river by average person according to FES (1972). 
POPULATION DOSE-COMMITMENT ESTIMATES AND

AVERAGE INDIVIDUAL DOSE-COMMITMENT HISTOGRAM FOR

E. I. HATCH 1 AND 2

Dose Commitments (person-rem) from Waterborne Pathways

Total Body GI-LLI $\underline{\text { Thyroid }}$ Bone Liver

$\begin{array}{llllll}\text { Infant } & 0.0 \mathrm{E}+00 & 0.0 \mathrm{E}+00 & 0.0 \mathrm{E}+00 & 0.0 \mathrm{E}+00 & 0.0 \mathrm{E}+00 \\ \text { Child } & 2.8 \mathrm{E}-01 & 1.6 \mathrm{E}-02 & 3.5 \mathrm{E}-03 & 1.3 \mathrm{E}+00 & 1.5 \mathrm{E}+00 \\ \text { Teen } & 5.2 \mathrm{E}-01 & 3.2 \mathrm{E}-02 & 2.5 \mathrm{E}-03 & 7.7 \mathrm{E}-01 & 1.3 \mathrm{E}+01 \\ \text { Adult } & 5.6 \mathrm{E}+00 & 2.8 \mathrm{E}-01 & 1.7 \mathrm{E}-02 & 4.5 \mathrm{E}+00 & 7.7 \mathrm{E}+00 \\ \text { TOTAL } & 6.4 \mathrm{E}+00 & 3.3 \mathrm{E}-01 & 2.3 \mathrm{E}-02 & 6.5 \mathrm{E}+00 & 1.1 \mathrm{E}+01\end{array}$

Dose Commitments (person-rem) from Airborne Pathways

Total Body GI-LLI Thyroid Bone Liver

$\begin{array}{lllllll}\text { Infant } & 5.0 \mathrm{E}-04 & 5.1 \mathrm{E}-04 & 3.9 \mathrm{E}-03 & 4.9 \mathrm{E}-04 & 6.3 \mathrm{E}-04 & 5.2 \mathrm{E}-04 \\ \text { Child } & 5.9 \mathrm{E}-03 & 5.6 \mathrm{E}-03 & 2.4 \mathrm{E}-02 & 5.2 \mathrm{E}-03 & 6.6 \mathrm{E}-03 & 6.1 \mathrm{E}-03 \\ \text { Teen } & 4.1 \mathrm{E}-03 & 4.0 \mathrm{E}-03 & 1.1 \mathrm{E}-02 & 3.4 \mathrm{E}-03 & 4.4 \mathrm{E}-03 & 4.5 \mathrm{E}-03 \\ \text { Adult } & 2.4 \mathrm{E}-02 & 2.4 \mathrm{E}-02 & 4.7 \mathrm{E}-02 & 2.02-02 & 2.5 \mathrm{E}-02 & 2.5 \mathrm{E}-02 \\ \text { TOTAL } & 3.5 \mathrm{E}-02 & 3.4 \mathrm{E}-02 & 8.6 \mathrm{E}-02 & 2.9 \mathrm{E}-02 & 3.6 \mathrm{E}-02 & 3.6 \mathrm{E}-02\end{array}$

Production/Consumption factors:

Produce: 0.13 Milk: $1.5 \quad$ Meat: 2.9

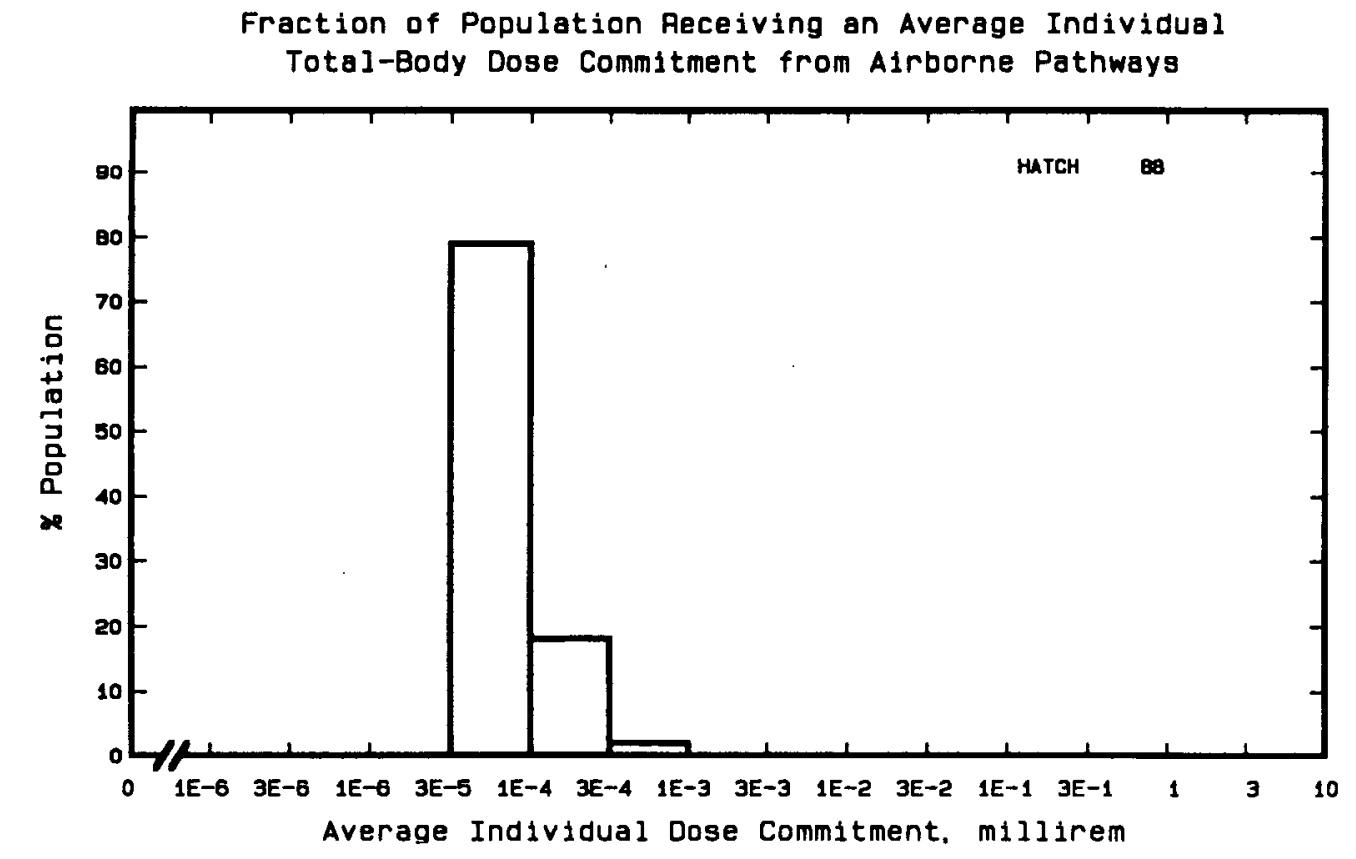


Site: HOPE CREEK

SALEM COUNTY, NEW JERSEY

Location: $\quad$ N $39.5733^{\circ}$ W $75.4667^{\circ}$

POPULATION DATA

Total Population Within 2-to-80-km Region: 4.9E6

Major Metropolitan Centers Within Region:

Center

Population

Location

Philadelphia

Camden

$1,700,000$

88,000

Wilmington

75,000

Vineland

55,000

Chester

46,000

$64 \mathrm{~km} \quad \mathrm{NNE}$

$61 \mathrm{~km} \quad \mathrm{NE}$

$30 \mathrm{~km} \mathrm{~N}$

$38 \mathrm{~km} \cdot \mathrm{E}$

$43 \mathrm{~km} \quad \mathrm{NNE}$

SITE-SPECIFIC DATA - AIRBORNE PATHWAYS

Average Annual State Production

Veg: $7.4 \mathrm{E} 7$ kilogram

of Crops and Animal Products

In 80-km Radius Circle

Milk: 2.7E8 liter

Meat: 2.4E7 kilogram

Regional Productivity Factor:

0.8

Animal Grazing Factor:

0.6

Meteorology Period of Record: 1 JAN 77 - 31 DEC 81 Recovery: $97 \%$

SITE-SPECIFIC DATA - WATERBORNE PATHWAYS via DELAWARE RIVER and BAY

Average River Flow

at Site: $16,000 \mathrm{ft}^{3} / \mathrm{s}$

Drinking Water: None

Fish:

Edible Harvest: $3.6 \mathrm{E} 5 \mathrm{~kg} / \mathrm{yr}$

Dilution Factor: 1

Invertebrates:

Edible Harvest: $1.6 \mathrm{E} 5 \mathrm{~kg} / \mathrm{yr}$

Dilution Factor: 1 


\section{POPULATION DOSE-COMMITMENT ESTIMATES AND \\ AVERAGE INDIVIDUAL DOSE-COMMITMENT HISTOGRAM FOR \\ HOPE CREEK}

Dose Commitments (person-rem) from Waterborne Pathways

Total Body GI-LLI Ihyroid Bone Liver

$\begin{array}{llllll}\text { Infant } & 0.0 \mathrm{E}+00 & 0.0 \mathrm{E}+00 & 0.0 \mathrm{E}+00 & 0.0 \mathrm{E}+00 & 0.0 \mathrm{E}+00 \\ \text { Child } & 1.7 \mathrm{E}-01 & 5.5 \mathrm{E}-02 & 7.8 \mathrm{E}-06 & 1.5 \mathrm{E}-01 & 2.8 \mathrm{E}-01 \\ \text { Teen } & 1.1 \mathrm{E}-01 & 1.2 \mathrm{E}-01 & 6.2 \mathrm{E}-06 & 9.4 \mathrm{E}-02 & 2.5 \mathrm{E}-01 \\ \text { Adult } & 6.8 \mathrm{E}-01 & 1.1 \mathrm{E}+00 & 4.5 \mathrm{E}-05 & 6.2 \mathrm{E}-01 & 1.6 \mathrm{E}+00 \\ \text { TOTAL } & 9.6 \mathrm{E}-01 & 1.2 \mathrm{E}+00 & 5.9 \mathrm{E}-05 & 8.5 \mathrm{E}-01 & 2.1 \mathrm{E}+00\end{array}$

Dose Commitments (person-rem) from Airborne Pathways

Total Body GI-LLI Ihyroid Bone Liver Lung

$\begin{array}{lllllll}\text { Infant } & 1.5 \mathrm{E}-03 & 1.5 \mathrm{E}-03 & 1.5 \mathrm{E}-03 & 1.2 \mathrm{E}-04 & 1.5 \mathrm{E}-03 & 1.5 \mathrm{E}-03 \\ \text { Child } & 2.3 \mathrm{E}-02 & 2.3 \mathrm{E}-02 & 2.3 \mathrm{E}-02 & 1.4 \mathrm{E}-03 & 2.3 \mathrm{E}-02 & 2.3 \mathrm{E}-02 \\ \text { Teen } & 1.6 \mathrm{E}-02 & 1.6 \mathrm{E}-02 & 1.6 \mathrm{E}-02 & 1.0 \mathrm{E}-03 & 1.6 \mathrm{E}-02 & 1.6 \mathrm{E}-02 \\ \text { Adult } & 9.1 \mathrm{E}-02 & 9.1 \mathrm{E}-02 & 9.1 \mathrm{E}-02 & 6.1 \mathrm{E}-03 & 9.1 \mathrm{E}-02 & 9.1 \mathrm{E}-02 \\ \text { TOTAL } & 1.3 \mathrm{E}-01 & 1.3 \mathrm{E}-01 & 1.3 \mathrm{E}-01 & 8.6 \mathrm{E}-03 & 1.3 \mathrm{E}-01 & 1.3 \mathrm{E}-01\end{array}$

Production/Consumption factors:

Produce: 0.062 Milk: 0.34 Meat: 0.050

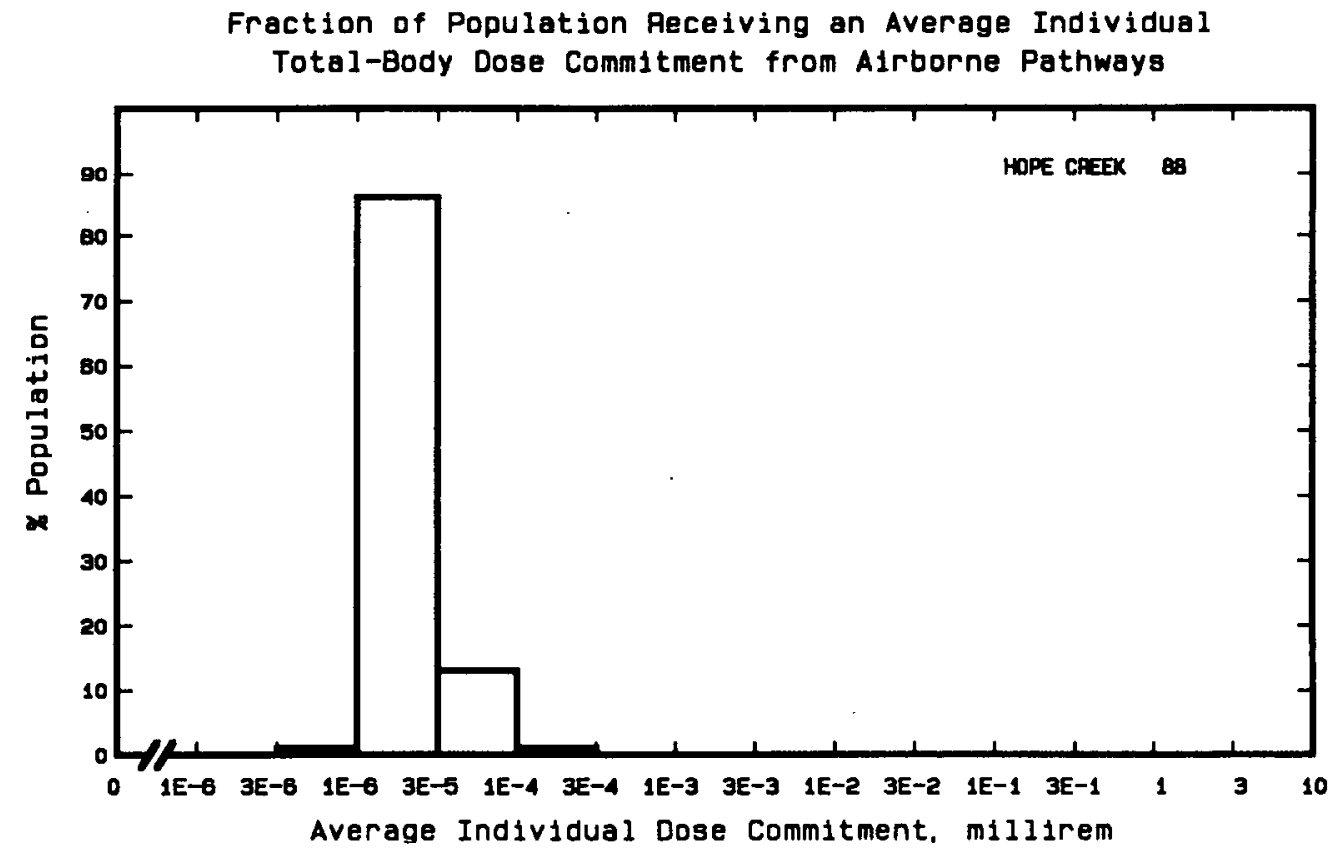


Site: INDIAN POINT

BUCHANAN, NEW YORK

Location: $\quad N 41.2714^{\circ} \quad$ W $73.9525^{\circ}$

POPULATION DATA

Total Population Within 2-to-80-km Region: 1.6E7

Major Metropolitan Centers Within Region:

Center

New York SMSA

Newark SMSA

Nassau County SMSA

Jersey City SMSA

Paterson-Clifton-Passaic SMSA
Population

$8,700,000$

$1,900,000$

$1,300,000$

570,000

450,000
Location

$57 \mathrm{~km} \quad \mathrm{~S}$

$62 \mathrm{~km} \quad \mathrm{SSW}$

$70 \mathrm{~km} \quad$ SSE

$61 \mathrm{~km} \quad \mathrm{~S}$

$44 \mathrm{~km} \quad \mathrm{SSW}$

SITE-SPECIFIC DATA - AIRBORNE PATHWAYS

Average Annual State Production

of Crops and Animal Products

In $80-\mathrm{km}$ Radius Circle

Regional Productivity Factor:

Animal Grazing Factor:
Veg: 7.6E7 kilogram

Milk: 7.0E8 liter

Meat: $3.3 E 7$ kilogram

0.8

0.5

Meteorology Period of Record: I JAN 75 - 31 DEC 75 Recovery: $96 \%$

SITE-SPECIFIC DATA - WATERBORNE PATHWAYS via HUDSON RIVER

Average River Flow

at Site: $20,000 \mathrm{ft}^{3} / \mathrm{s}$

Drinking Water:

None

Fish:

Edible Harvest: (a)

Dilution Factor: 0.001 (b)

(a) No fish catch data given in FES (1982), so generic consumption rates used (Table A-1).

(b) One percent of population obtain 10\% of the ir fish from river according to FES (1972). 
POPULATION DOSE-COMMITMENT ESTIMATES AND

AVERAGE INDIVIDUAL DOSE-COMMITMENT HISTOGRAM FOR

INDIAN POINT 1,2 , AND 3

Dose Commitments (person-rem) from Waterborne Pathways

Total Body GI-LLI Thyroid Bone Liver

$\begin{array}{llllll}\text { Infant } & 0.0 \mathrm{E}+00 & 0.0 \mathrm{E}+00 & 0.0 \mathrm{E}+00 & 0.0 \mathrm{E}+00 & 0.0 \mathrm{E}+00 \\ \text { Child } & 4.1 \mathrm{E}-03 & 2.7 \mathrm{E}-03 & 9.3 \mathrm{E}-04 & 2.4 \mathrm{E}-02 & 2.2 \mathrm{E}-02 \\ \text { Teen } & 7.5 \mathrm{E}-03 & 5.6 \mathrm{E}-03 & 6.8 \mathrm{E}-04 & 1.4 \mathrm{E}-02 & 1.9 \mathrm{E}-02 \\ \text { Adult } & 8.0 \mathrm{E}-02 & 4.9 \mathrm{E}-02 & 4.5 \mathrm{E}-03 & 8.3 \mathrm{E}-02 & 1.1 \mathrm{E}-01 \\ \text { TOTAL } & 9.2 \mathrm{E}-02 & 5.7 \mathrm{E}-02 & 6.1 \mathrm{E}-03 & 1.2 \mathrm{E}-01 & 1.5 \mathrm{E}-01\end{array}$

Dose Commitments (person-rem) from Airborne Pathways

Total Body GI-LLI Ihyroid Bone Liver Lung

$\begin{array}{lllllll}\text { Infant } & 4.8 \mathrm{E}-03 & 4.7 \mathrm{E}-03 & 1.5 \mathrm{E}-02 & 4.9 \mathrm{E}-03 & 5.3 \mathrm{E}-03 & 6.4 \mathrm{E}-03 \\ \text { Child } & 5.5 \mathrm{E}-02 & 5.4 \mathrm{E}-02 & 1.2 \mathrm{E}-01 & 5.3 \mathrm{E}-02 & 5.8 \mathrm{E}-02 & 8.1 \mathrm{E}-02 \\ \text { Teen } & 4.0 \mathrm{E}-01 & 4.0 \mathrm{E}-02 & 7.5 \mathrm{E}-02 & 3.7 \mathrm{E}-02 & 4.2 \mathrm{E}-02 & 6.6 \mathrm{E}-02 \\ \text { Adult } & 2.4 \mathrm{E}-01 & 2.4 \mathrm{E}-01 & 3.9 \mathrm{E}-01 & 2.2 \mathrm{E}-01 & 2.4 \mathrm{E}-01 & 3.4 \mathrm{E}-01 \\ \text { TOTAL } & 3.4 \mathrm{E}-01 & 3.4 \mathrm{E}-01 & 6.0 \mathrm{E}-01 & 3.1 \mathrm{E}-01 & 3.5 \mathrm{E}-01 & 4.9 \mathrm{E}-01\end{array}$

Production/Consumption factors:

Produce: 0.020 Milk: 0.27 Meat: 0.021

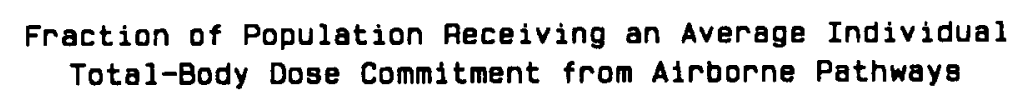
Total-Body Dose Commitment from Alrborne Pathways

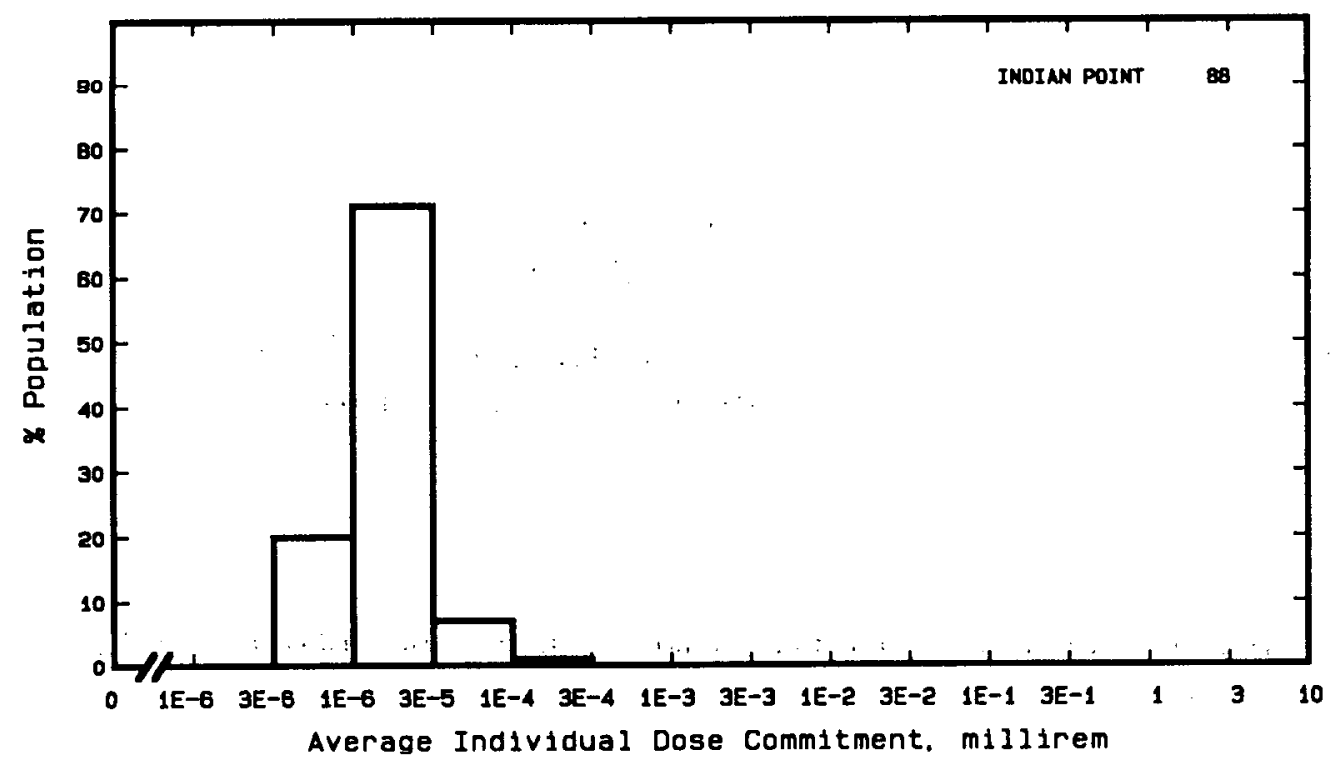


Site: KEWAUNEE

CARLTON, WISCONSIN

Location: $\quad$ N $44.3431^{\circ}$ W $87.5361^{\circ}$

POPULATION DATA

Total Population Within 2-to-80-km Region: $6.3 \mathrm{E5}$

Major Metropolitan Centers Within Region:

Center

Greenbay SMSA

Appleton SMSA

Sheboygan

Manitowoc

Neenah
Population

180,000

290,000

48,000

33,000

22,000
Location

$44 \mathrm{~km} \quad \mathrm{NW}$

$72 \mathrm{~km} \quad W$

$65 \mathrm{~km} \quad \mathrm{SSW}$

$29 \mathrm{~km} \quad \mathrm{SSW}$

$72 \mathrm{~km} \quad \mathrm{~W}$

SITE-SPECIFIC DATA - AIRBORNE PATHWAYS

Average Annual State Production

of Crops and Animal Products

In $80-\mathrm{km}$ Radius Circle

Regional Productivity Factor:

Animal Grazing Factor:
Veg: $7.2 \mathrm{E} 7 \mathrm{kilogram}$

Milk: 1.2E9 liter

Meat: 1.0E8 kilogram

0.5

0.5

Meteorology Period of Record: 2 JAN 69 - 31 DEC 69 Recovery: 76\%

Average Dilution Flow from

Plant: $44 \mathrm{ft}^{3} / \mathrm{s}$

Drinking Water:

Fish:
Exposed Population: 270,000

Dilution Factor: $8.2 \mathrm{E}-3(\mathrm{a})$

Edible Harvest: $1.1^{(\mathrm{b})} \mathrm{kg} / \mathrm{yr}$

(a) Drinking water dilution factor est imated by averaging di lution factors derived from FES (1972) suitably weighted for populations.

(b) Average individual consumption rate as given in FES used in lieu of catch data.

(c) Dilution factor reduced $1 / 10$ from that used in FES in consideration of lake mixing. 
POPULATION DOSE-COMMITMENT ESTIMATES AND

AVERAGE INDIVIDUAL DOSE-COMMITMENT HISTOGRAM FOR

KEWAUNEE

Dose Commitments (person-rem) from Waterborne Pathways

Total Body GI-LLI Ihyroid Bone Liver

$\begin{array}{llllll}\text { Infant } & 1.5 \mathrm{E}-02 & 1.5 \mathrm{E}-02 & 1.7 \mathrm{E}-02 & 1.6 \mathrm{E}-03 & 1.6 \mathrm{E}-02 \\ \text { Child } & 1.9 \mathrm{E}-01 & 1.9 \mathrm{E}-01 & 1.8 \mathrm{E}-01 & 1.0 \mathrm{E}-01 & 2.8 \mathrm{E}-01 \\ \text { Teen } & 1.0 \mathrm{E}-01 & 9.7 \mathrm{E}-02 & 6.7 \mathrm{E}-02 & 5.7 \mathrm{E}-02 & 1.6 \mathrm{E}-01 \\ \text { Adult } & 9.5 \mathrm{E}-01 & 8.4 \mathrm{E}-01 & 5.6 \mathrm{E}-01 & 3.3 \mathrm{E}-01 & 1.1 \mathrm{E}+00 \\ \text { TOTAL } & 1.3 \mathrm{E}+00 & 1.1 \mathrm{E}+00 & 8.2 \mathrm{E}-01 & 5.0 \mathrm{E}-01 & 1.5 \mathrm{E}+00\end{array}$

Dose Commitments (person-rem) from Airborne Pathways

Total Body GI-LLI Thyroid Bone Liver Lung

$\begin{array}{lllllll}\text { Infant } & 2.9 \mathrm{E}-05 & 2.8 \mathrm{E}-05 & 7.8 \mathrm{E}-05 & 2.5 \mathrm{E}-06 & 2.9 \mathrm{E}-05 & 2.9 \mathrm{E}-05 \\ \text { Child } & 4.5 \mathrm{E}-04 & 4.4 \mathrm{E}-04 & 8.0 \mathrm{E}-04 & 6.1 \mathrm{E}-05 & 4.4 \mathrm{E}-04 & 4.5 \mathrm{E}-04 \\ \text { Teen } & 2.7 \mathrm{E}-04 & 2.7 \mathrm{E}-04 & 4.3 \mathrm{E}-04 & 3.6 \mathrm{E}-05 & 2.7 \mathrm{E}-04 & 2.7 \mathrm{E}-04 \\ \text { Adult } & 1.5 \mathrm{E}-03 & 1.5 \mathrm{E}-03 & 2.1 \mathrm{E}-03 & 1.9 \mathrm{E}-04 & 1.5 \mathrm{E}-03 & 1.5 \mathrm{E}-03 \\ \text { TOTAL } & 2.2 \mathrm{E}-03 & 2.2 \mathrm{E}-03 & 3.4 \mathrm{E}-03 & 2.9 \mathrm{E}-04 & 2.2 \mathrm{E}-03 & 2.2 \mathrm{E}-03\end{array}$

Production/Consumption factors:

Produce: 0.30 Milk: $7.1 \quad$ Meat: 1.0

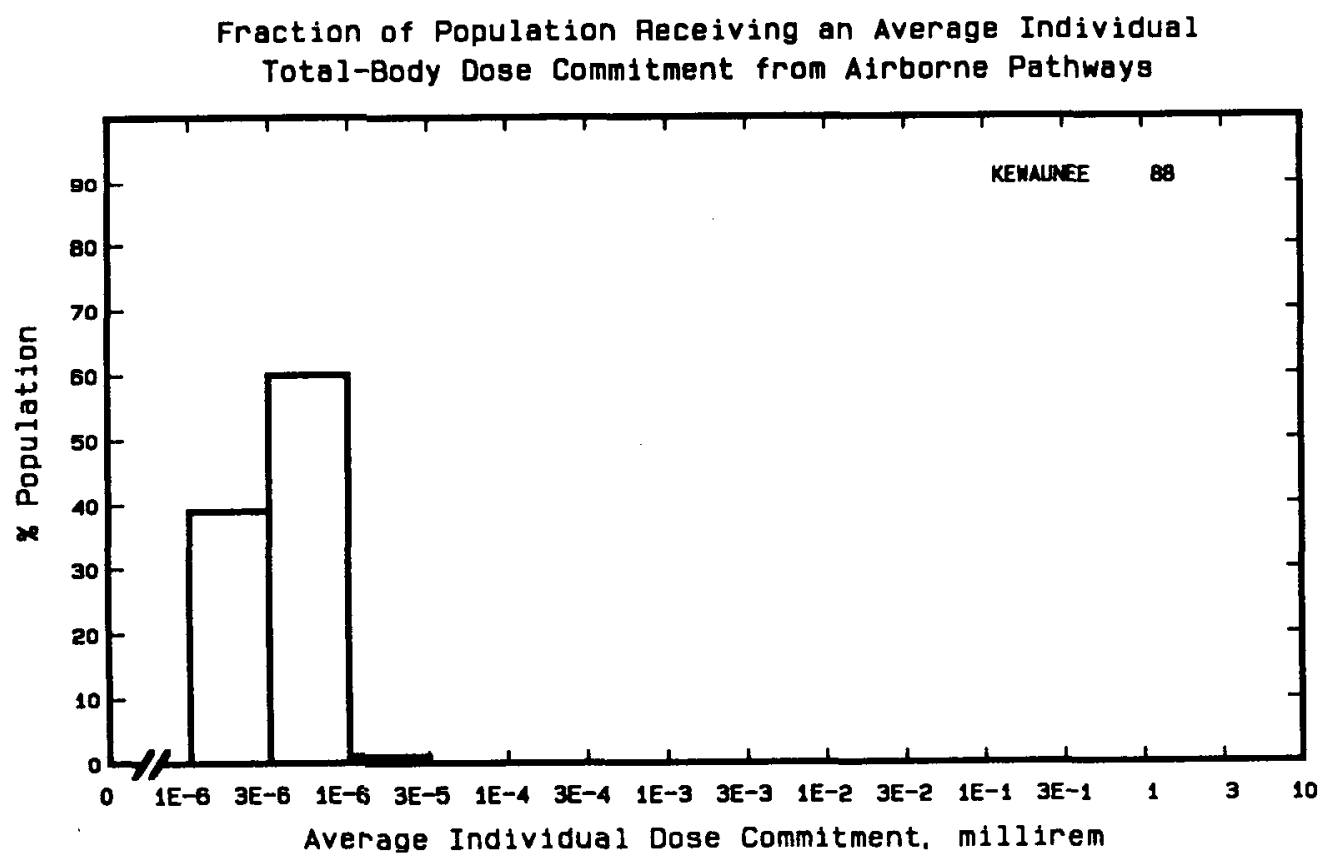


Site: LACROSSE

GENOA, WISCONSIN

Location: $\quad$ N $43.5583^{\circ} \quad$ W $91.2306^{\circ}$

POPULATION DATA

Total Population Within 2-to-80-km Region: 3.5E5

Major Metropolitan Centers Within Region:

Center

La Crosse SMSA

Winona
Population

94,000

25,000
Location

$27 \mathrm{~km} \quad \mathrm{~N}$

$64 \mathrm{~km} \quad$ WNW

SITE-SPECIFIC DATA - AIRBORNE PATHWAYS

Average Annual State Production

of Crops and Animal Products

In $80-\mathrm{km}$ Radius Circle

Regional Productivity Factor:

Animal Grazing Factor:
Veg: $7.2 \mathrm{E} 7$ kilogram

Mi1k: 1.2E9 liter

Meat: $1.0 \mathrm{E} 8$ kilogram

1

0.5

Meteorology Period of Record: 1 JAN 75 - 31 DEC 75 Recovery: 97\%

Average River

Flow at Site: $28,000 \mathrm{ft}^{3} / \mathrm{s}$

Drinking Water:

None

Fish:

Edible Harvest: (a)

Dilution Factor: 0.5 (a)

(a) No fish catch data given in FES (1976), so $1 / 2$ population assumed to consume river fish at generic consumption rates (Table A-1). 


\section{POPULATION DOSE-COMMITMENT ESTIMATES AND \\ AVERAGE INDIVIDUAL DOSE-COMMITMENT HISTOGRAM FOR}

\section{LACROSSE}

Dose Commitments (person-rem) from Waterborne Pathways

Total Body GI-LLI $\underline{\text { Thyroid }}$ Bone Liver

$\begin{array}{llllll}\text { Infant } & 0.0 \mathrm{E}+00 & 0.0 \mathrm{E}+00 & 0.0 \mathrm{E}+00 & 0.0 \mathrm{E}+00 & 0.0 \mathrm{E}+00 \\ \text { Child } & 1.7 \mathrm{E}-02 & 1.9 \mathrm{E}-03 & 2.1 \mathrm{E}-06 & 1.2 \mathrm{E}-01 & 1.1 \mathrm{E}-01 \\ \text { Teen } & 3.3 \mathrm{E}-02 & 3.9 \mathrm{E}-03 & 1.9 \mathrm{E}-06 & 6.9 \mathrm{E}-02 & 9.1 \mathrm{E}-02 \\ \text { Adult } & 3.6 \mathrm{E}-01 & 3.4 \mathrm{E}-02 & 1.5 \mathrm{E}-05 & 4.0 \mathrm{E}-01 & 5.3 \mathrm{E}-01 \\ \text { TOTAL } & 4.1 \mathrm{E}-01 & 4.0 \mathrm{E}-02 & 1.9 \mathrm{E}-05 & 5.8 \mathrm{E}-01 & 7.3 \mathrm{E}-01\end{array}$

Dose Commitments (person-rem) from Airborne Pathways

Total Body GI-LLI Thyroid $\underline{\text { Bone Liver Lung }}$

$\begin{array}{lllllll}\text { Infant } & 2.4 \mathrm{E}-06 & 2.4 \mathrm{E}-06 & 2.4 \mathrm{E}-06 & 9.9 \mathrm{E}-08 & 2.5 \mathrm{E}-06 & 2.5 \mathrm{E}-06 \\ \text { Child } & 5.7 \mathrm{E}-05 & 5.7 \mathrm{E}-05 & 5.7 \mathrm{E}-05 & 1.6 \mathrm{E}-06 & 5.8 \mathrm{E}-05 & 5.8 \mathrm{E}-05 \\ \text { Teen } & 3.4 \mathrm{E}-05 & 3.4 \mathrm{E}-05 & 3.4 \mathrm{E}-05 & 5.9 \mathrm{E}-07 & 3.4 \mathrm{E}-05 & 3.4 \mathrm{E}-05 \\ \text { Adult } & 1.8 \mathrm{E}-04 & 1.8 \mathrm{E}-04 & 1.8 \mathrm{E}-04 & 2.4 \mathrm{E}-06 & 1.8 \mathrm{E}-04 & 1.8 \mathrm{E}-04 \\ \text { TOTAL } & 2.8 \mathrm{E}-04 & 2.8 \mathrm{E}-04 & 2.8 \mathrm{E}-04 & 4.7 \mathrm{E}-06 & 2.8 \mathrm{E}-04 & 2.8 \mathrm{E}-04\end{array}$

Production/Consumption factors:

Produce: $1.0 \quad$ Milk: $25 \quad$ Meat: 3.6

Fraction of Population Receiving an Average Individual Total-Body Dose Commitment from Airborne Pathways

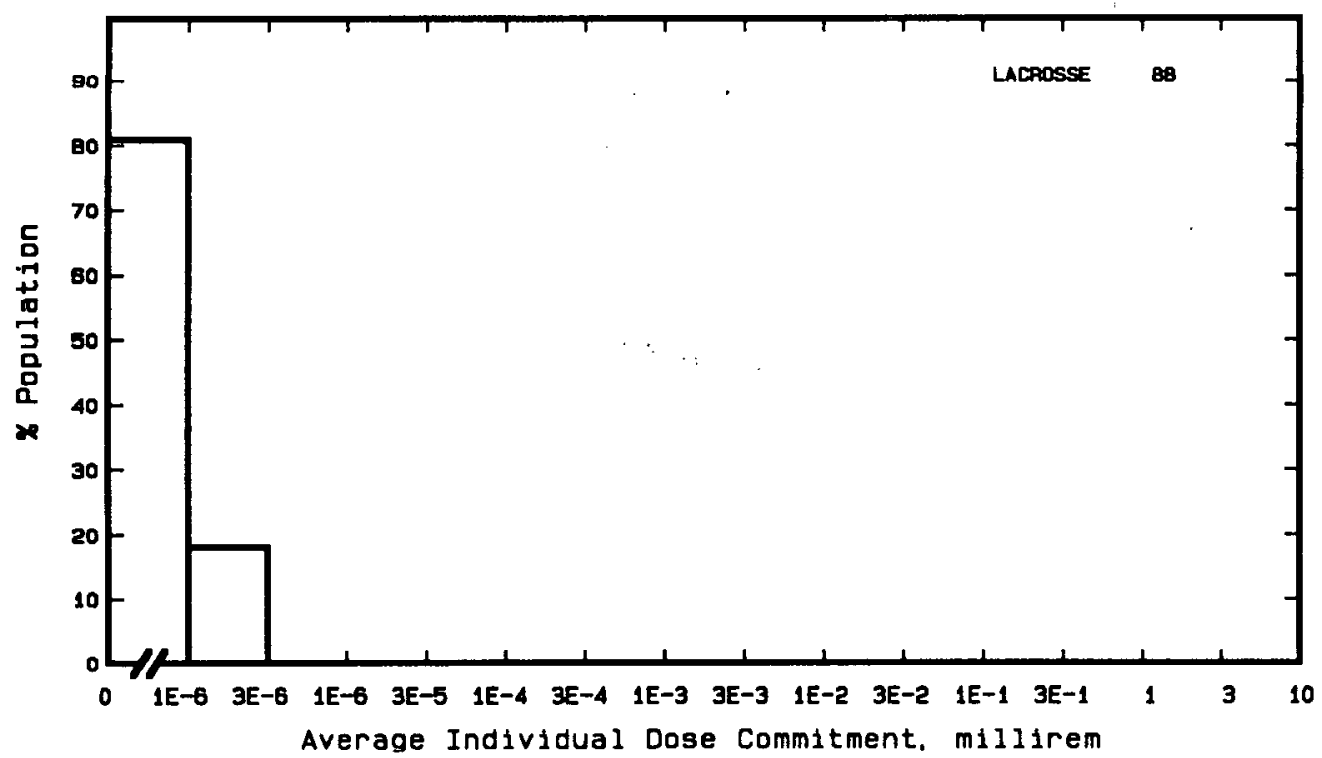


Site: LASALLE

SENECA, ILLINOIS

Location: N $41.2439^{\circ} \quad$ W $88.6708^{\circ}$

POPULATION DATA

Total Population Within 2-to-80-km Region: 1.1E6

Major Metropolitan Centers Within Region:

Center

Joliet

Aurora

Kankakee

Dekalb

Naperville
Population

78,000

80,000

100,000

33,000

42,000
Location

$59 \mathrm{~km} \quad \mathrm{NNE}$

$65 \mathrm{~km}$ NNE

$69 \mathrm{~km} \quad \mathrm{ESE}$

$77 \mathrm{~km} \mathrm{~N}$

$73 \mathrm{~km} \quad \mathrm{NE}$

SITE-SPECIFIC DATA - AIRBORNE PATHWAYS

Average Annual State Production

of Crops and Animal Products

In $80-\mathrm{km}$ Radius Circle

Regional Productivity Factor:

Animal Grazing Factor:
Veg: $1.1 \mathrm{E} 8$ kilogram

Milk: 1.8 E8 liter

Meat: $1.9 \mathrm{E} 8$ kilogram

Meteorology Period of Record: 1 Jan 82 - 31 Dec 82 Recovery: $99 \%$

Drinking Water: None

Fish:

None ${ }^{(a)}$

(a) Because of river quality, no fish consumption is considered according to FES (1973). 
POPULATION DOSE-COMMITMENT ESTIMATES AND

AVERAGE INDIVIDUAL DOSE-COMMITMENT HISTOGRAM FOR

LASALLE 1 AND 2

Dose Commitments (person-rem) from Waterborne Pathways

Total Body GI-LII $\underline{\text { Thyroid Bone Liver }}$

Infant

Child

Teen

Adult

(Little or No Waterborne Pathway Doses)

TOTAL

Dose Commitments (person-rem) from Airborne Pathways

Iotal Body GI-LII Thyroid Bone Liver

$\begin{array}{lllllll}\text { Infant } & 3.9 \mathrm{E}-03 & 3.9 \mathrm{E}-03 & 1.1 \mathrm{E}-02 & 3.9 \mathrm{E}-03 & 3.9 \mathrm{E}-03 & 4.0 \mathrm{E}-03 \\ \text { Child } & 4.4 \mathrm{E}-02 & 4.4 \mathrm{E}-02 & 1.0 \mathrm{E}-01 & 4.4 \mathrm{E}-02 & 4.4 \mathrm{E}-02 & 4.5 \mathrm{E}-02 \\ \text { Teen } & 3.2 \mathrm{E}-02 & 3.2 \mathrm{E}-02 & 5.7 \mathrm{E}-02 & 3.2 \mathrm{E}-02 & 3.2 \mathrm{E}-02 & 3.4 \mathrm{E}-02 \\ \text { Aduit } & 1.9 \mathrm{E}-01 & 1.9 \mathrm{E}-01 & 2.9 \mathrm{E}-01 & 1.9 \mathrm{E}-01 & 1.9 \mathrm{E}-01 & 2.0 \mathrm{E}-01 \\ \text { TOTAL } & 2.7 \mathrm{E}-01 & 2.7 \mathrm{E}-01 & 4.6 \mathrm{E}-01 & 2.7 \mathrm{E}-01 & 2.7 \mathrm{E}-01 & 2.8 \mathrm{E}-01\end{array}$

Production/Consumption factors:

Produce: $0.49 \quad$ Milk: $1.1 \quad$ Meat: 2.0

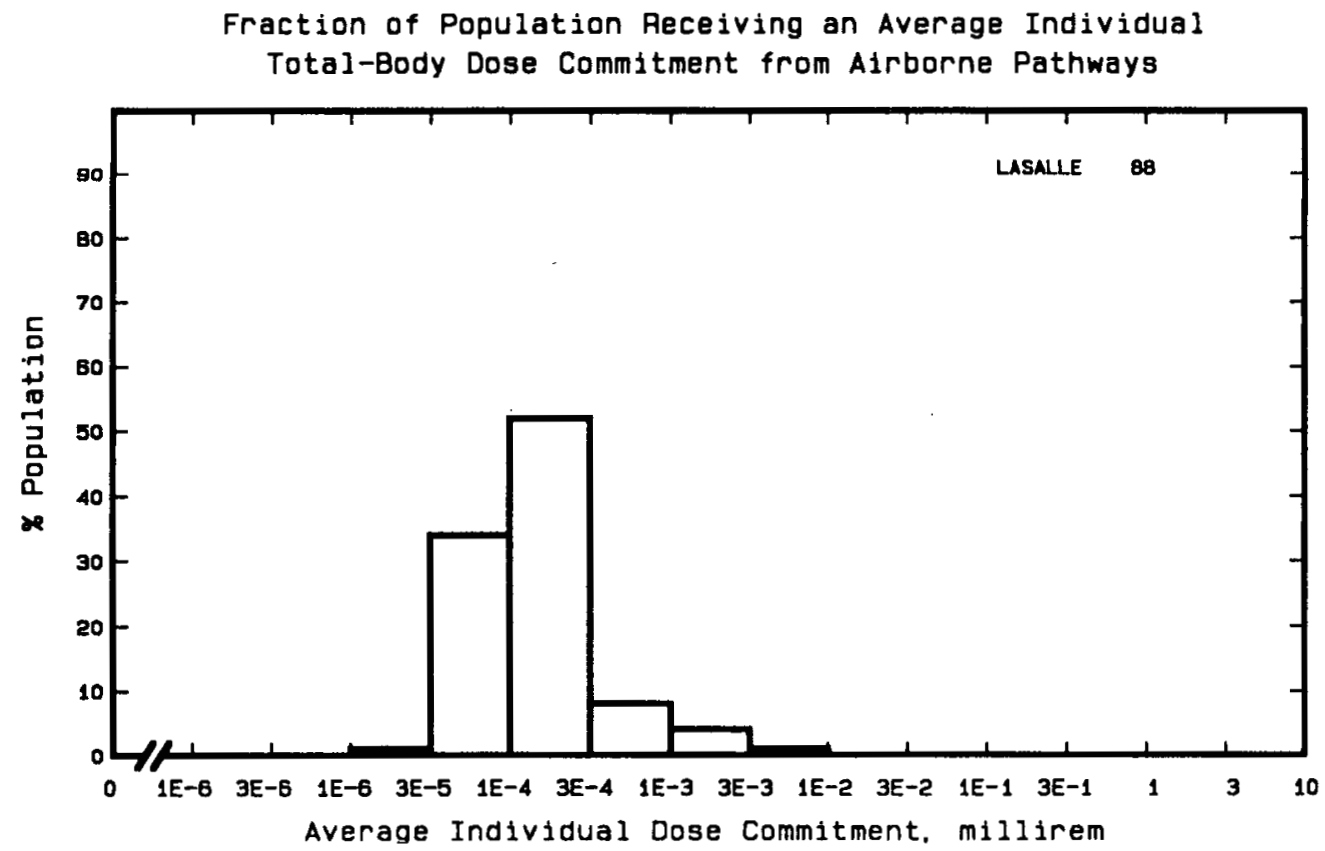


Site: LIMERICK

POTTSTOWN, PENNSYLVANIA

Location: $\quad N 40.2242^{\circ}$

W $75.5875^{\circ}$

POPULATION DATA

Total Population Within 2-to-80-km Region: 6.9E6

Major Metropolitan Centers Within Region:

Center

Philadelphia PMSA

Al lentown-Bethl ehem MSA

Reading MSA

Lancaster MSA

Wilmington PMSA

Camdon

Trenton PMSA
Population

$$
\begin{array}{r}
4,800,000 \\
66,000 \\
310,000 \\
360,000 \\
550,000 \\
110,000 \\
310,000
\end{array}
$$

\section{Location}

$\begin{array}{lll}40 \mathrm{~km} & \text { ESE } \\ 42 \mathrm{~km} & \text { ENE } \\ 34 \mathrm{~km} & \text { WNW } \\ 68 \mathrm{~km} & \text { WSW } \\ 56 \mathrm{~km} & \text { S } \\ 52 \mathrm{~km} & \text { SE } \\ 71 \mathrm{~km} & \text { E }\end{array}$

\section{SITE-SPECIFIC DATA - AIRBORNE PATHWAYS}

Average Annual State Production

of Crops and Animal Products

In $80-\mathrm{km}$ Radius Circle

Regional Productivity Factor:

Animal Grazing Factor:
Veg: $5.3 E 7$ kilogram

Milk: 5.3E8 liter

Meat: $5.4 E 7$ kilogram

0.9

0.6

Meteorology Period of Record: 1 Jan $74-31$ Dec 74 Recovery: $93 \%$

\section{SITE-SPECIFIC DATA - WATERBORNE PATHWAYS via SCHUYLKILL RIVER}

Average River Flow

at site: $3,000 \mathrm{ft}^{3} \mathrm{~s}$

Drinking Water:

None

Fish:

None 


\section{POPULATION DOSE-COMMITMENT ESTIMATES AND \\ AVERAGE INDIVIDUAL DOSE-COMMITMENT HISTOGRAM FOR}

LIMERICK

Dose Commitments (person-rem) from Waterborne Pathways

Total Body GI-LII $\quad$ Thyroid Bone Liver

Infant

Child

Teen

Adult

TOTAL

(No Waterborne Pathway Doses) (a)

Dose Commitments (person-rem) from Airborne Pathways

Total Body GI-LII Thyroid Bone Liver lung

$\begin{array}{lllllll}\text { Infant } & 3.1 \mathrm{E}-03 & 3.1 \mathrm{E}-03 & 1.8 \mathrm{E}-02 & 1.1 \mathrm{E}-03 & 3.1 \mathrm{E}-03 & 3.1 \mathrm{E}-03 \\ \text { Child } & 4.2 \mathrm{E}-02 & 4.2 \mathrm{E}-02 & 1.3 \mathrm{E}-01 & 1.2 \mathrm{E}-02 & 4.2 \mathrm{E}-02 & 4.2 \mathrm{E}-02 \\ \text { Teen } & 3.1 \mathrm{E}-02 & 3.1 \mathrm{E}-02 & 7.2 \mathrm{E}-02 & 8.7 \mathrm{E}-03 & 3.1 \mathrm{E}-02 & 3.2 \mathrm{E}-02 \\ \text { Adult } & 1.8 \mathrm{E}-01 & 1.8 \mathrm{E}-01 & 3.4 \mathrm{E}-01 & 5.2 \mathrm{E}-02 & 1.8 \mathrm{E}-01 & 1.8 \mathrm{E}-01 \\ \text { TOTAL } & 2.5 \mathrm{E}-01 & 2.5 \mathrm{E}-01 & 5.6 \mathrm{E}-01 & 7.4 \mathrm{E}-02 & 2.5 \mathrm{E}-01 & 2.6 \mathrm{E}-01\end{array}$

Production/Consumption factors:

Produce: $0.036 \quad$ Milk: $0.53 \quad$ Meat: 0.089

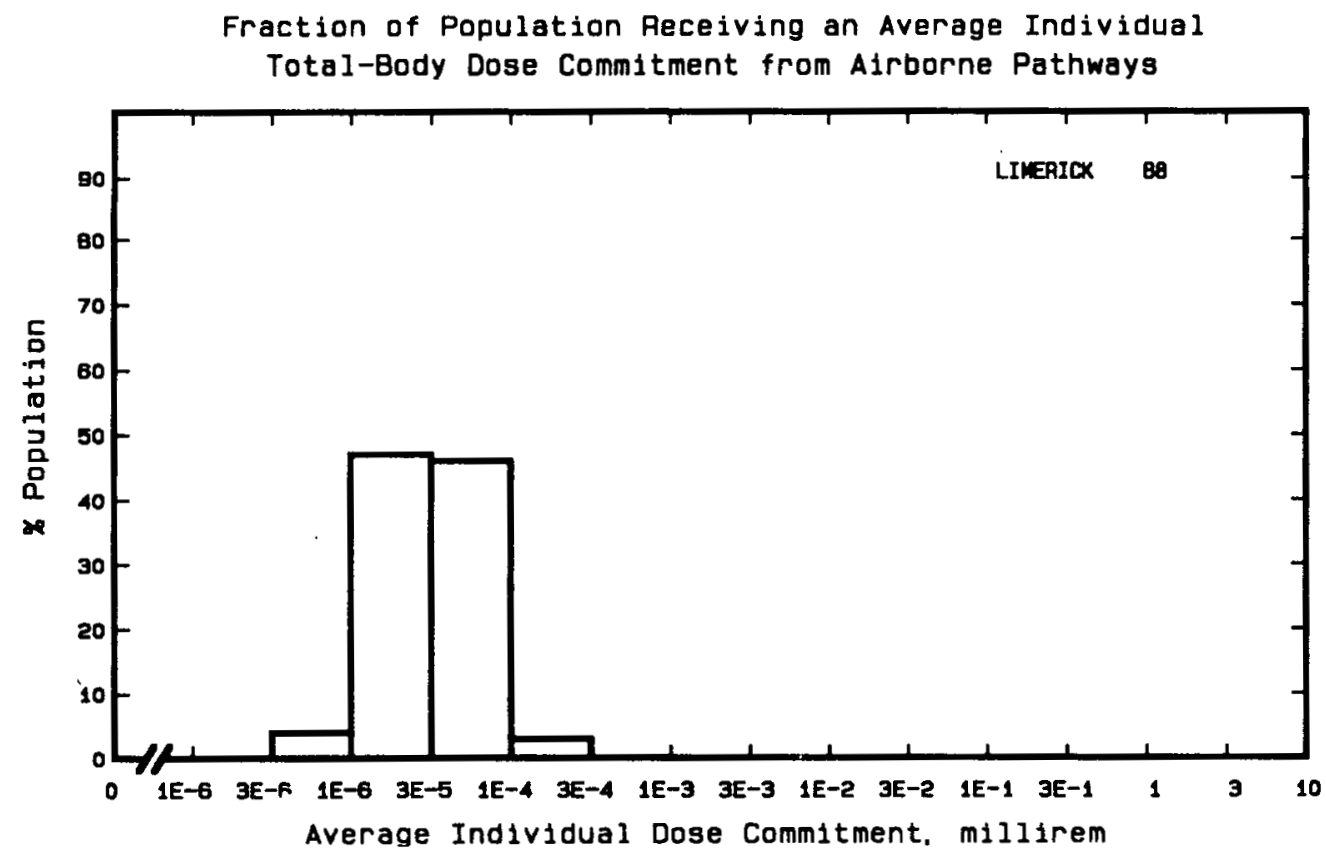

(a) No liquid releases reported (Tichler, et al., 1991). 
Site: MAINE YANKEE

Location: N $43.9506^{\circ}$
LINCOLN COUNTY, MAINE

W $69.6961^{0}$

POPULATION DATA

Total Population Within 2-to-80-km Region: 6.3 E5

Major Metropolitan Centers Within Region:

\begin{tabular}{lrrrr} 
Center & Population & \multicolumn{2}{c}{ Location } \\
\cline { 2 - 5 } & & & \\
Portland SMSA & 180,000 & $56 \mathrm{~km}$ & WSW \\
Lewiston-Auburn SMSA & 76,000 & $45 \mathrm{~km}$ & WNW \\
Augusta & 22,000 & $41 \mathrm{~km}$ & $\mathrm{~N}$ \\
Biddeford & 20,000 & $80 \mathrm{~km}$ & SW \\
Waterville & 18,000 & $67 \mathrm{~km}$ & $\mathrm{~N}$
\end{tabular}

SITE-SPECIFIC DATA - AIRBORNE PATHWAYS

Average Annual State Production

of Crops and Animal Products

In $80-\mathrm{km}$ Radius Circle

Regional Productivity Factor:

Animal Grazing Factor:
Veg: 2.4E8 kilogram

Milk: $6.6 \mathrm{E} 7$ liter

Meat: $4.3 \mathrm{E} 6$ kilogram

0.6

0.5

Meteorology Period of Record: 1 APR 75 - 31 MAR 76 Recovery: 98\%

Average Dilution Flow

from Plant: $760 \mathrm{ft}^{3} / \mathrm{s}$

Fish:

Edible Harvest: (a)

Dilution Factor: 0.001

Invertebrates:

Edible Harvest: (a)

Dilution Factor: 0.002

(a) No seafood harvest data given in FES (1972). thus generic population consumption rates used (Table $A-1$ ). 
POPULATION DOSE-COMMITMENT ESTIMATES AND

AVERAGE INDIVIDUAL DOSE-COMMITMENT HISTOGRAM FOR

MAINE YANKEE

Dose Commitments (person-rem) from Waterborne Pathways

Total Body GI-LLI Thyroid Bone Liver

$\begin{array}{llllll}\text { Infant } & 0.0 \mathrm{E}+00 & 0.0 \mathrm{E}+00 & 0.0 \mathrm{E}+00 & 0.0 \mathrm{E}+00 & 0.0 \mathrm{E}+00 \\ \text { Child } & 4.5 \mathrm{E}-04 & 9.8 \mathrm{E}-04 & 7.9 \mathrm{E}-05 & 1.7 \mathrm{E}-03 & 1.0 \mathrm{E}-03 \\ \text { Teen } & 3.1 \mathrm{E}-04 & 2.0 \mathrm{E}-03 & 6.0 \mathrm{E}-05 & 9.4 \mathrm{E}-04 & 7.9 \mathrm{E}-04 \\ \text { Adult } & 2.1 \mathrm{E}-03 & 1.7 \mathrm{E}-02 & 4.2 \mathrm{E}-04 & 5.5 \mathrm{E}-03 & 4.6 \mathrm{E}-03 \\ \text { TOTAL } & 2.9 \mathrm{E}-03 & 2.0 \mathrm{E}-02 & 5.6 \mathrm{E}-04 & 8.1 \mathrm{E}-03 & 6.5 \mathrm{E}-03\end{array}$

Dose Commitments (person-rem) from Airborne Pathways

Total Body GI-LLI Ihyroid Bone Liver Lung

$\begin{array}{lllllll}\text { Infant } & 4.4 \mathrm{E}-05 & 4.4 \mathrm{E}-05 & 1.2 \mathrm{E}-04 & 2.2 \mathrm{E}-05 & 4.4 \mathrm{E}-05 & 4.6 \mathrm{E}-05 \\ \text { Child } & 9.4 \mathrm{E}-04 & 9.4 \mathrm{E}-04 & 2.1 \mathrm{E}-03 & 2.5 \mathrm{E}-04 & 9.4 \mathrm{E}-04 & 9.7 \mathrm{E}-04 \\ \text { Teen } & 5.8 \mathrm{E}-04 & 5.8 \mathrm{E}-04 & 1.1 \mathrm{E}-03 & 1.8 \mathrm{E}-04 & 5.8 \mathrm{E}-04 & 6.2 \mathrm{E}-04 \\ \text { Adult } & 3.2 \mathrm{E}-03 & 3.2 \mathrm{E}-03 & 5.1 \mathrm{E}-03 & 1.1 \mathrm{E}-03 & 3.2 \mathrm{E}-03 & 3.3 \mathrm{E}-03 \\ \text { TOTAL } & 4.7 \mathrm{E}-03 & 4.7 \mathrm{E}-03 & 8.5 \mathrm{E}-03 & 1.5 \mathrm{E}-03 & 4.7 \mathrm{E}-03 & 4.9 \mathrm{E}-03\end{array}$

Production/Consumption factors:

Produce: $1.2 \quad$ Milk: $0.48 \quad$ Meat: 0.051

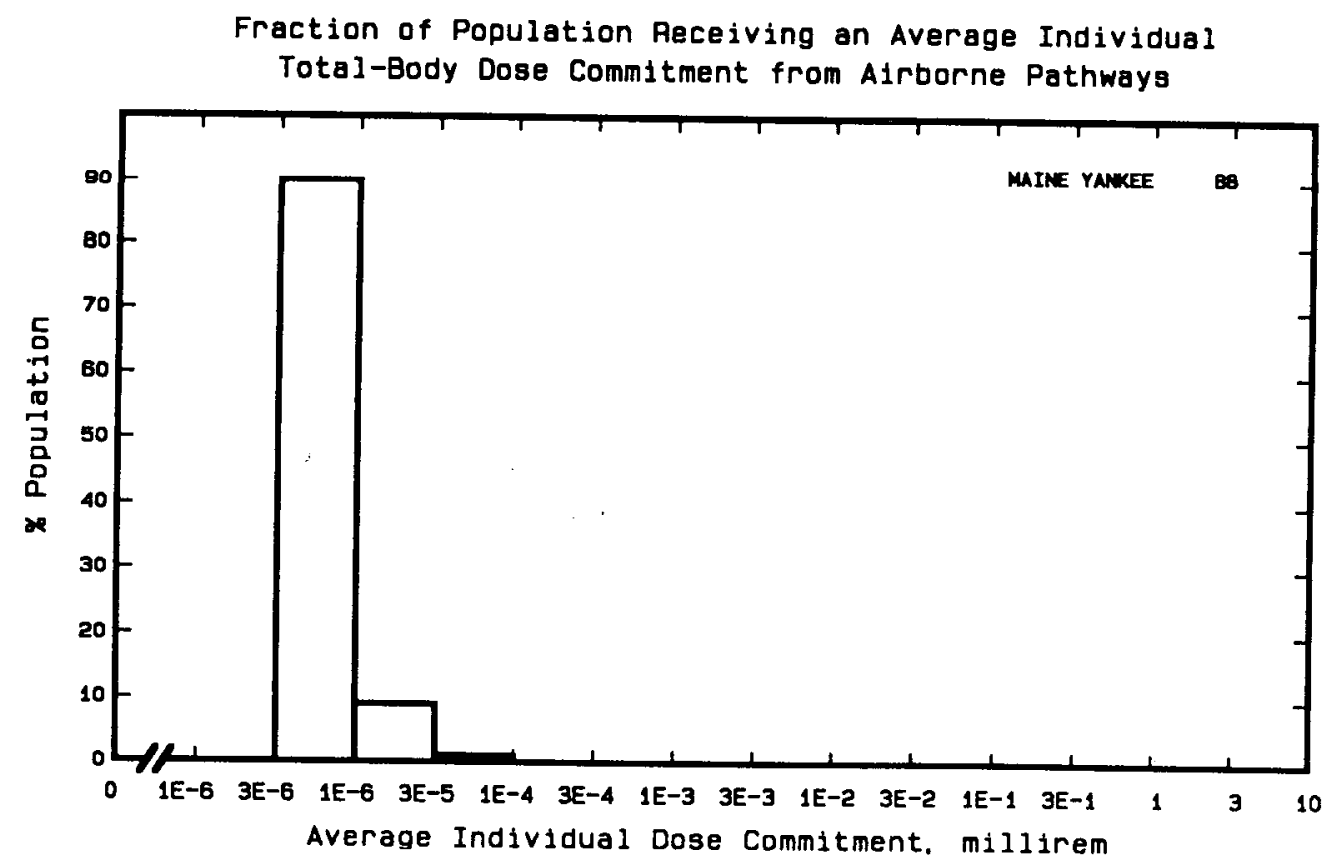


Site: McGUIRE

CORNELIUS, NORTH CAROLINA

Location: $\quad N 35.4322^{\circ} \quad$ W $80.9483^{\circ}$

POPULATION DATA

Total Population Within 2-to-80-km Region: 1.8E6

Major Metropolitan Centers Within Region:

Center

Charlotte-Gastonia SMSA Rockhil1

Kannapolis

Sal isbury

Hickory
Population

700,000

37,000

37,000

25,000

23,000
Location

$25 \mathrm{~km} \quad \mathrm{~S}$

$57 \mathrm{~km} \quad \mathrm{~S}$

$30 \mathrm{~km} \quad \mathrm{E}$

$51 \mathrm{~km} \quad$ ENE

$49 \mathrm{~km} \quad \mathrm{NW}$

\section{SITE-SPECIFIC DATA - AIRBORNE PATHWAYS}

Average Annual State Production

of Crops and Animal Products

In $80-\mathrm{km}$ Radius Circle

Regional Productivity Factor:

Animal Grazing Factor:
Veg: 2.6E7 kilogram

Milk: 1.0E8 liter

Meat: $5.8 \mathrm{E} 7$ kilogram

Meteorology Period of Record: 17 OCT 70 - 16 OCT 71 Recovery: 90\%

SITE-SPECIFIC DATA - WATERBORNE PATHWAYS via LAKE NORMAN On CATAWBA

RIVER

Average River Flow at Site: $2,600 \mathrm{ft}^{3} / \mathrm{s}$

Drinking Water:

Exposed Population: 770,000

Fish:

Edible Harvest: $2.0 \mathrm{E} 5(\mathrm{a}) \mathrm{kg} / \mathrm{yr}$

Dilution Factor: 1

(a) Edible harvest from downstream reservoirs derived from creel surveys obtained from James R. Thornton, Duke Power Company. 
POPULATION DOSE-COMMITMENT ESTIMATES AND

AVERAGE INDIVIDUAL DOSE-COMMITMENT HISTOGRAM FOR

MCGUIRE 1 AND 2

Dose Commitments (person-rem) from Waterborne Pathways

- Iotal Body GI-LLI Thyroid Bone Liver

$\begin{array}{llllll}\text { Infant } & 3.0 \mathrm{E}-01 & 3.1 \mathrm{E}-01 & 1.6 \mathrm{E}+00 & 3.1 \mathrm{E}-02 & 3.1 \mathrm{E}-01 \\ \text { Child } & 3.4 \mathrm{E}+00 & 4.1 \mathrm{E}+00 & 1.2 \mathrm{E}+01 & 4.8 \mathrm{E}-01 & 3.5 \mathrm{E}+00 \\ \text { Teen } & 1.3 \mathrm{E}+00 & 2.4 \mathrm{E}+00 & 4.0 \mathrm{E}+00 & 1.6 \mathrm{E}-01 & 1.4 \mathrm{E}+00 \\ \text { Adult } & 1.1 \mathrm{E}+01 & 2.2 \mathrm{E}+01 & 3.0 \mathrm{E}+01 & 9.8 \mathrm{E}-01 & 1.1 \mathrm{E}+01 \\ \text { TOTAL } & 1.6 \mathrm{E}+01 & 2.8 \mathrm{E}+01 & 4.8 \mathrm{E}+01 & 1.7 \mathrm{E}+00 & 1.7 \mathrm{E}+01\end{array}$

Dose Commitments (person-rem) from Airborne Pathways

Total Body GI-LLI Thyroid Bone Liver Lung

$\begin{array}{lllllll}\text { Infant } & 2.4 \mathrm{E}-03 & 2.4 \mathrm{E}-03 & 1.1 \mathrm{E}-02 & 2.2 \mathrm{E}-03 & 2.4 \mathrm{E}-03 & 2.6 \mathrm{E}-03 \\ \text { Child } & 2.8 \mathrm{E}-02 & 2.8 \mathrm{E}-02 & 7.9 \mathrm{E}-02 & 2.4 \mathrm{E}-02 & 2.8 \mathrm{E}-02 & 3.0 \mathrm{E}-02 \\ \text { Teen } & 2.0 \mathrm{E}-02 & 2.0 \mathrm{E}-02 & 4.1 \mathrm{E}-02 & 1.7 \mathrm{E}-02 & 2.0 \mathrm{E}-02 & 2.3 \mathrm{E}-02 \\ \text { Adult } & 1.2 \mathrm{E}-01 & 1.2 \mathrm{E}-01 & 2.0 \mathrm{E}-01 & 1.1 \mathrm{E}-01 & 1.2 \mathrm{E}-01 & 1.3 \mathrm{E}-01 \\ \text { TOTAL } & 1.7 \mathrm{E}-01 & 1.7 \mathrm{E}-01 & 3.3 \mathrm{E}-01 & 1.5 \mathrm{E}-01 & 1.7 \mathrm{E}-01 & 1.9 \mathrm{E}-01\end{array}$

Production/Consumption factors:

Produce: $0.067 \quad$ Milk: $0.40 \quad$ Meat: 0.37

Fraction of Population Receiving an Average Individual

Total-Body Dose Commitment from Alrborne Pathways

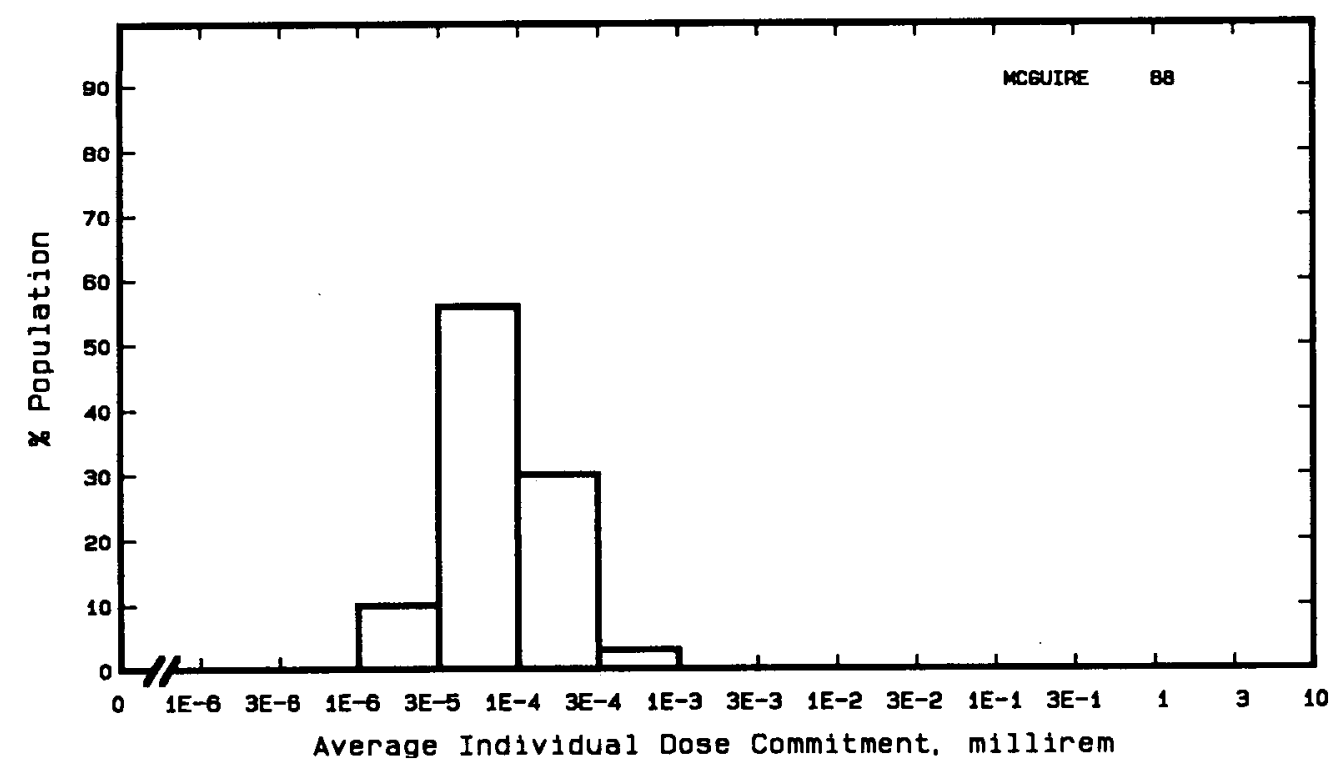


Site: MILLSTONE

WATERFORD, CONNECTICUT

Location: $\quad$ N $41.3086^{\circ} \quad$ W $72.1681^{\circ}$

\section{POPULATION DATA}

Total Population Within 2-to-80-km Region: 2.6E6

Major Metropolitan Centers Within Region:

Center

Hartford SMSA

New Haven-West Haven SMSA

New London-Norwich SMSA

New Britain SMSA

Waterbury SMSA
Population

750,000

430,000

250,000

140,000

230,000
Location

$67 \mathrm{~km} \quad \mathrm{NW}$

$64 \mathrm{~km} \quad W$

$8 \mathrm{~km} \quad \mathrm{NNE}$

$65 \mathrm{~km} \quad \mathrm{NW}$

$78 \mathrm{~km}$ WNW

SITE-SPECIFIC DATA - AIRBORNE PATHWAYS

Average Annual State Production

of Crops and Animal Products

In $80-\mathrm{km}$ Radius Circle

Regional Productivity Factor:

Animal Grazing Factor:
Veg: $3.2 E 7$ kilogram

Milk: 4.4E8 liter

Meat: $2.0 E 7$ kilogram

0.6

0.6

Meteorology Period of Record: 1 JAN 74 - 31 DEC 74 Recovery: 95\%

Fish:

Invertebrates:
Average Dilution Flow

from all 3 Plants: $3,900 \mathrm{ft}^{3} / \mathrm{s}$

Edible Harvest: $9.1 \mathrm{E} 4 \mathrm{~kg} / \mathrm{yr}$

Dilution Factor: 0.001

Edible Harvest: $9.1 \mathrm{E} 4 \mathrm{~kg} / \mathrm{yr}$

Dilution Factor: 0.002 
POPULATION DOSE-COMMITMENT ESTIMATES AND AVERAGE INDIVIDUAL DOSE-COMMITMENT HISTOGRAM FOR

MILLSTONE 1,2 , AND 3

Dose Commitments (person-rem) from Waterborne Pathways

Total Body GI-LLI Ihyroid Bone Liver

$\begin{array}{llllll}\text { Infant } & 0.0 \mathrm{E}+00 & 0.0 \mathrm{E}+00 & 0.0 \mathrm{E}+00 & 0.0 \mathrm{E}+00 & 0.0 \mathrm{E}+00 \\ \text { Child } & 4.3 \mathrm{E}-04 & 7.1 \mathrm{E}-04 & 2.4 \mathrm{E}-04 & 6.0 \mathrm{E}-04 & 5.5 \mathrm{E}-04 \\ \text { Teen } & 3.0 \mathrm{E}-04 & 1.5 \mathrm{E}-03 & 1.7 \mathrm{E}-04 & 3.4 \mathrm{E}-04 & 4.5 \mathrm{E}-04 \\ \text { Adult } & 1.9 \mathrm{E}-03 & 1.3 \mathrm{E}-02 & 1.1 \mathrm{E}-03 & 2.0 \mathrm{E}-03 & 2.7 \mathrm{E}-03 \\ \text { TOTAL } & 2.7 \mathrm{E}-03 & 1.5 \mathrm{E}-02 & 1.5 \mathrm{E}-03 & 3.0 \mathrm{E}-03 & 3.7 \mathrm{E}-03\end{array}$

Dose Commitments (person-rem) from Airborne Pathways

Total Body GI-LLI Thyroid Bone Liver Lung

$\begin{array}{lllllll}\text { Infant } & 3.4 \mathrm{E}-03 & 3.4 \mathrm{E}-03 & 9.4 \mathrm{E}-02 & 1.9 \mathrm{E}-03 & 3.7 \mathrm{E}-03 & 3.4 \mathrm{E}-03 \\ \text { Child } & 3.9 \mathrm{E}-02 & 3.8 \mathrm{E}-02 & 5.0 \mathrm{E}-01 & 2.0 \mathrm{E}-02 & 4.0 \mathrm{E}-02 & 4.0 \mathrm{E}-02 \\ \text { Teen } & 2.7 \mathrm{E}-02 & 2.7 \mathrm{E}-02 & 2.1 \mathrm{E}-01 & 1.4 \mathrm{E}-02 & 2.7 \mathrm{E}-02 & 2.8 \mathrm{E}-02 \\ \text { Adult } & 1.5 \mathrm{E}-01 & 1.5 \mathrm{E}-01 & 7.1 \mathrm{E}-01 & 8.0 \mathrm{E}-02 & 1.5 \mathrm{E}-01 & 1.6 \mathrm{E}-01 \\ \text { TOTAL } & 2.2 \mathrm{E}-01 & 2.2 \mathrm{E}-01 & 1.5 \mathrm{E}+00 & 1.2 \mathrm{E}-01 & 2.2 \mathrm{E}-01 & 2.3 \mathrm{E}-01\end{array}$

Production/Consumption factors:

Produce: $0.038 \quad$ Milk: 0.77 Meat: 0.059

Fraction of Population Receiving an Average Individual Total-Body Dose Commitment from Airborne Pathways

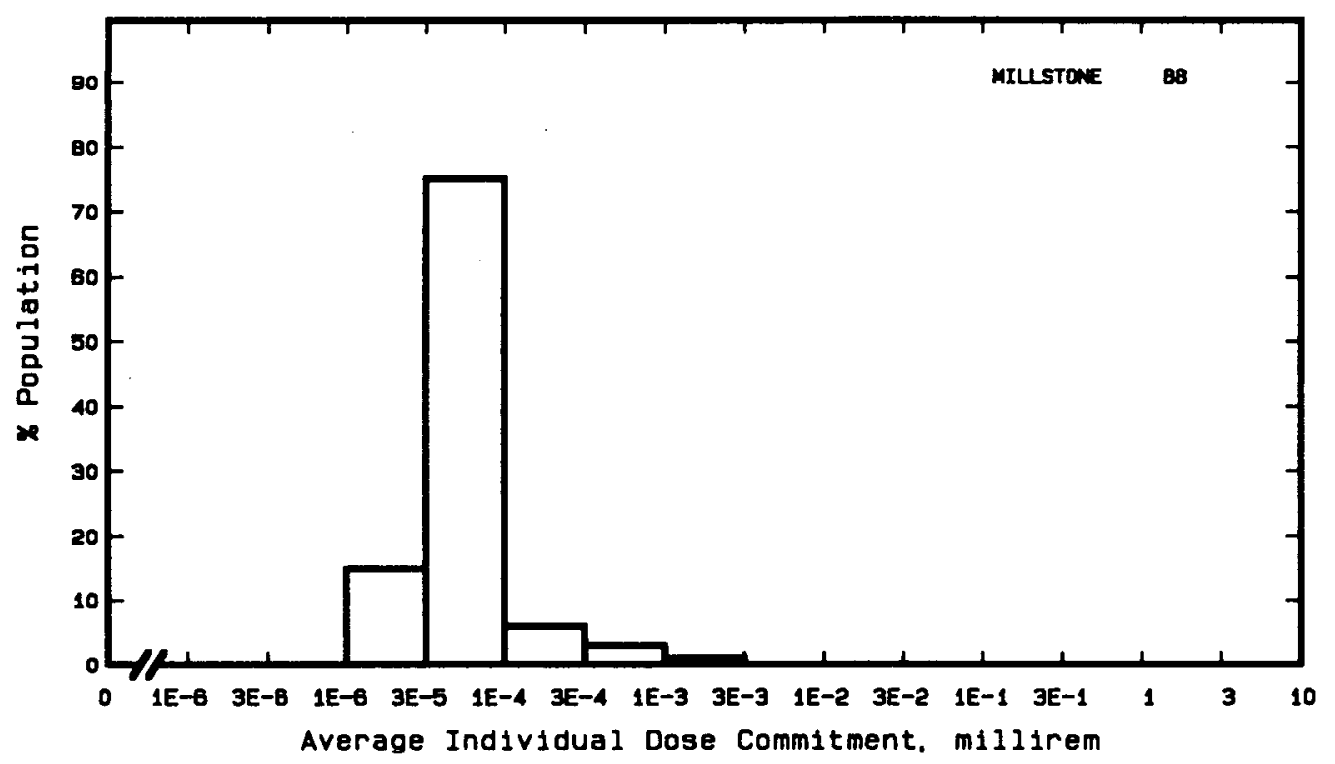


Site: MONTICELLO

Location: $\quad$ N $45.3333^{\circ} \quad$ W $93.8483^{\circ}$
MONTICELLO, MINNESOTA

POPULATION DATA

Total Population Within 2-to-80-km Region: 2.3E6

Major Metropolitan Centers Within Region:

Center Population Location

Minneapolis-St. Paul SMSA $2,100,000 \quad 60 \mathrm{~km} \quad$ SE

St. Cloud SMSA $\quad 160,000 \quad 36 \mathrm{~km}$ NW

Bloomington $\quad 85,000 \quad 72 \mathrm{~km}$ SE

Edina $\quad 48,000 \quad 63 \mathrm{~km} \quad S E$

$\begin{array}{llll}\text { Richfield } & 40,000 & 67 \mathrm{~km} & \mathrm{SE}\end{array}$

SITE-SPECIFIC DATA - AIRBORNE PATHWAYS

Average Annual State Production

of Crops and Animal Products

In $80-\mathrm{km}$ Radius Circle

Veg: $1.2 \mathrm{E} 8$ kilogram

Milk: $4.0 \mathrm{E} 8$ liter

Meat: $1.1 \mathrm{E} 8$ kilogram

Regional Productivity Factor:

Animal Grazing Factor:

1

0.5

Meteorology Period of Record: 1 JAN 74 - 31 DEC 74 Recovery: 92\%

SITE-SPECIFIC DATA - WATERBORNE PATHWAYS via MISSISSIPPI RIVER

Average River Flow

at Site: $4,600 \mathrm{ft}^{3} / \mathrm{s}$

Drinking Water:

Exposed Population: None

Fish:

Edible Harvest: None 


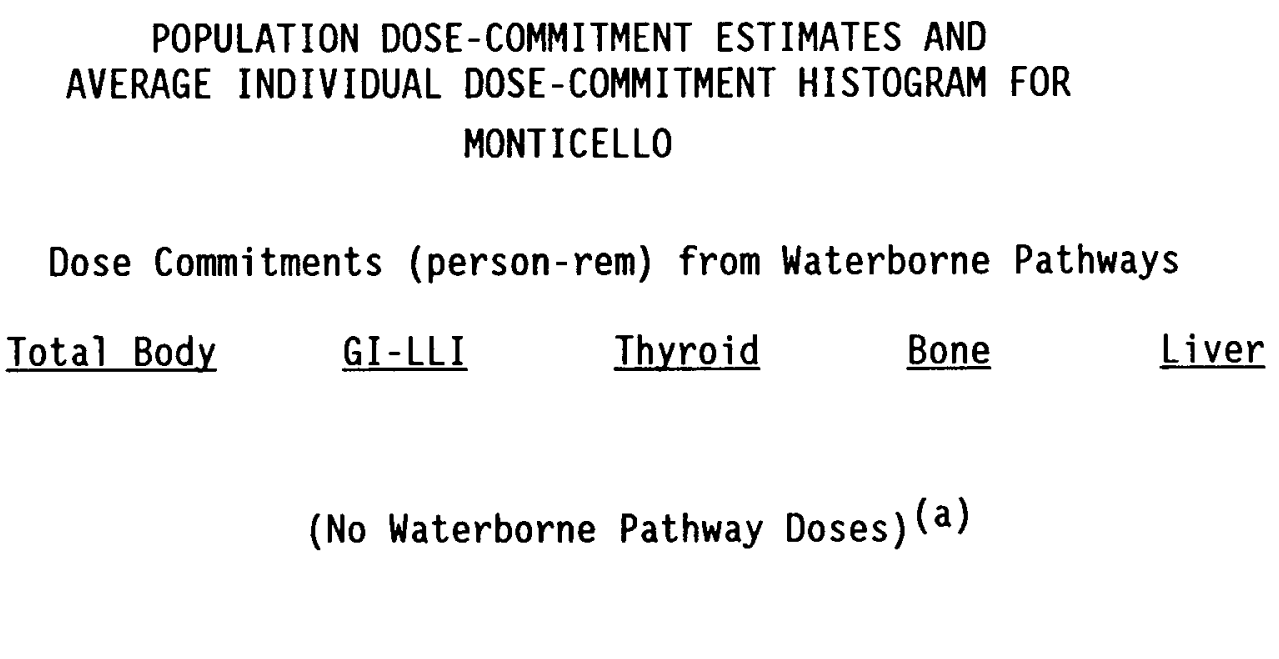

Dose Commitments (person-rem) from Airborne Pathways

Infant

Child

Teen

Adult

TOTAL

\section{Total Body GI-LLI Thyroid Bone Liver Lung}

$\begin{array}{lllllll}\text { Infant } & 2.7 \mathrm{E}-03 & 2.6 \mathrm{E}-03 & 6.7 \mathrm{E}-02 & 2.3 \mathrm{E}-03 & 2.9 \mathrm{E}-03 & 2.7 \mathrm{E}-03 \\ \text { Child } & 3.2 \mathrm{E}-02 & 3.1 \mathrm{E}-02 & 4.5 \mathrm{E}-01 & 2.7 \mathrm{E}-02 & 3.3 \mathrm{E}-02 & 3.2 \mathrm{E}-02 \\ \text { Teen } & 2.2 \mathrm{E}-02 & 2.2 \mathrm{E}-02 & 1.9 \mathrm{E}-01 & 1.8 \mathrm{E}-02 & 2.3 \mathrm{E}-02 & 2.3 \mathrm{E}-02 \\ \text { Adult } & 1.3 \mathrm{E}-01 & 1.3 \mathrm{E}-01 & 6.9 \mathrm{E}-01 & 1.0 \mathrm{E}-01 & 1.3 \mathrm{E}-01 & 1.3 \mathrm{E}-01 \\ \text { TOTAL } & 1.8 \mathrm{E}-01 & 1.8 \mathrm{E}-01 & 1.4 \mathrm{E}+00 & 1.5 \mathrm{E}-01 & 1.9 \mathrm{E}-01 & 1.9 \mathrm{E}-01\end{array}$

Production/Consumption factors:

Produce: $0.28 \quad$ Milk: $1.3 \quad$ Meat: 0.58

Fraction of Population Receiving an Averoge Individual

Total-Body Dose Commitment from Alrborne Pathways

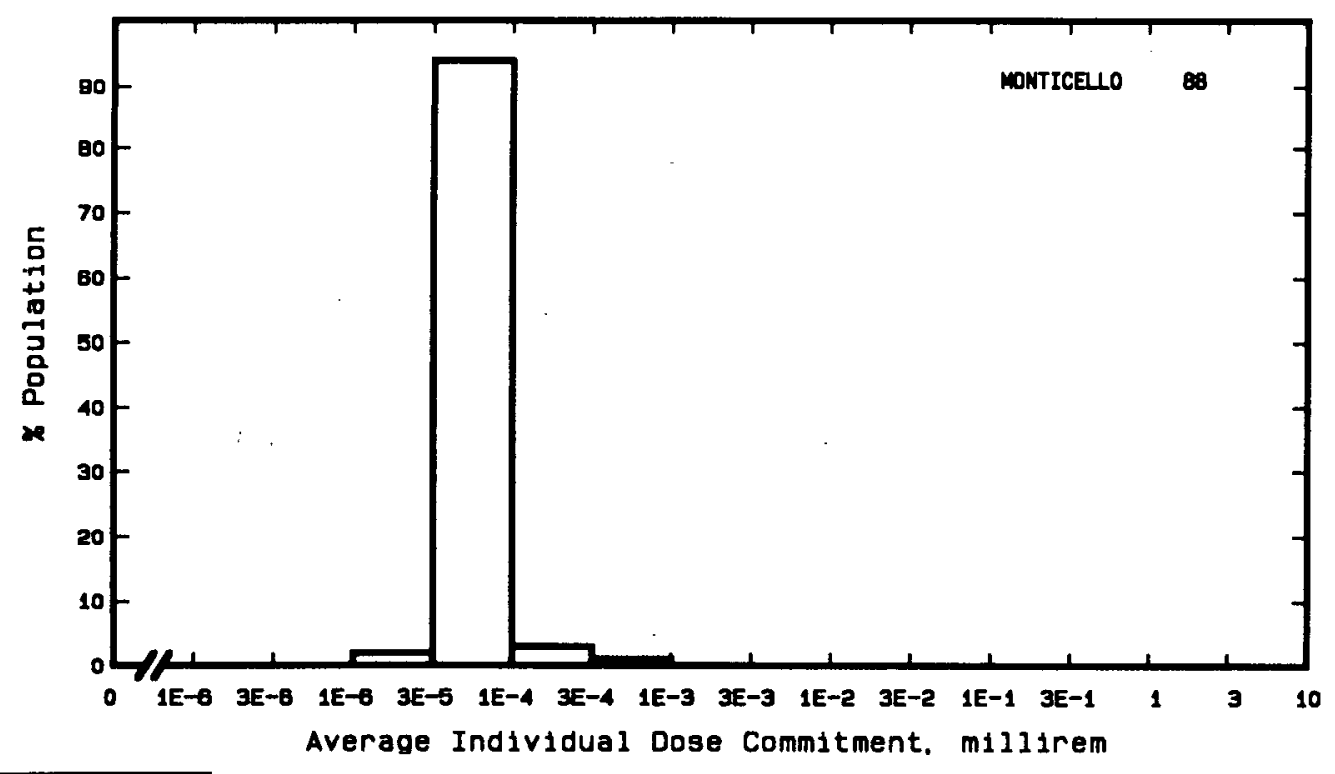

(a) No liquid releases reported (Tichler, et al. 1991). 
Site: NINE MILE POINT

OSWEGO, NEW YORK

Location: $\quad N 43.5222^{\circ}$ W $76.4100^{\circ}$

POPULATION DATA

Total Population Within 2-to-80-km Region: 8.6E5

Major Metropolitan Centers Within Region:

$\quad$ Center
Syracuse SMSA
Auburn
Watertown
Kingston
Oswego

Population

Location

Syracuse SMSA

680,000

33,000

28,000

24,000

20,000

$56 \mathrm{~km} \quad$ SSE

$67 \mathrm{~km} \quad \mathrm{SSW}$

$64 \mathrm{~km} \quad \mathrm{NE}$

$79 \mathrm{~km} \quad \mathrm{~N}$

$11 \mathrm{~km} \quad \mathrm{SW}$

SITE-SPECIFIC DATA - AIRBORNE PATHWAYS

Average Annual State Production

Veg: $7.6 \mathrm{E} 7 \mathrm{kilogram}$

of Crops and Animal Products

In $80-\mathrm{km}$ Radius Circle

Milk: 7.0E8 liter

Meat: $3.3 \mathrm{E} 7$ kilogram

Regional Productivity Factor:

Animal Grazing Factor:

0.7

0.5

Meteorology Period of Record: I JAN 74 - 31 DEC 75 Recovery: 97\%

SITE-SPECIFIC DATA - WATERBORNE PATHWAYS via LAKE ONTARIO

Drinking Water:

Average Dilution Flow

from Plant: $130 \mathrm{ft}^{3} / \mathrm{s}$

Drinking Water:

Exposed Population: 560,000

Dilution Factor: 0.01

Fish:

Edible Harvest: $7.3 \mathrm{E5} \mathrm{kg} / \mathrm{yr}$

Dilution Factor: 0.0033 
POPULATION DOSE-COMMITMENT ESTIMATES AND

AVERAGE INDIVIDUAL DOSE-COMMITMENT HISTOGRAM FOR

NINE MILE POINT 1 AND 2

Dose Commitments (person-rem) from Waterborne Pathways

Total Body GI-LLI $\underline{\text { Thyroid }}$ Bone Liver

$\begin{array}{llllll}\text { Infant } & 3.6 \mathrm{E}-03 & 4.5 \mathrm{E}-03 & 3.1 \mathrm{E}-04 & 1.1 \mathrm{E}-03 & 4.5 \mathrm{E}-03 \\ \text { Child } & 5.2 \mathrm{E}-02 & 7.0 \mathrm{E}-02 & 3.4 \mathrm{E}-03 & 1.8 \mathrm{E}-02 & 6.1 \mathrm{E}-02 \\ \text { Teen } & 2.1 \mathrm{E}-02 & 7.1 \mathrm{E}-02 & 1.3 \mathrm{E}-03 & 7.9 \mathrm{E}-03 & 3.5 \mathrm{E}-02 \\ \text { Adult } & 1.3 \mathrm{E}-01 & 6.7 \mathrm{E}-01 & 1.1 \mathrm{E}-02 & 5.4 \mathrm{E}-02 & 2.2 \mathrm{E}-01 \\ \text { TOTAL } & 2.1 \mathrm{E}-01 & 8.2 \mathrm{E}-01 & 1.6 \mathrm{E}-02 & 8.0 \mathrm{E}-02 & 3.2 \mathrm{E}-01\end{array}$

Dose Commitments (person-rem) from Airborne Pathways

Total Body GI-LLI Ihyroid Bone Liver Lung

$\begin{array}{lllllll}\text { Infant } & 6.3 \mathrm{E}-05 & 6.1 \mathrm{E}-05 & 7.5 \mathrm{E}-05 & 6.3 \mathrm{E}-05 & 9.4 \mathrm{E}-05 & 6.7 \mathrm{E}-05 \\ \text { Child } & 8.7 \mathrm{E}-04 & 8.1 \mathrm{E}-04 & 8.7 \mathrm{E}-04 & 9.3 \mathrm{E}-04 & 1.0 \mathrm{E}-03 & 8.7 \mathrm{E}-04 \\ \text { Teen } & 5.4 \mathrm{E}-04 & 5.3 \mathrm{E}-04 & 5.1 \mathrm{E}-04 & 4.3 \mathrm{E}-04 & 6.0 \mathrm{E}-04 & 5.7 \mathrm{E}-04 \\ \text { Adult } & 2.9 \mathrm{E}-03 & 2.9 \mathrm{E}-03 & 2.7 \mathrm{E}-03 & 2.0 \mathrm{E}-03 & 2.9 \mathrm{E}-03 & 2.9 \mathrm{E}-03 \\ \text { TOTAL } & 4.4 \mathrm{E}-03 & 4.3 \mathrm{E}-03 & 4.2 \mathrm{E}-03 & 3.5 \mathrm{E}-03 & 4.7 \mathrm{E}-03 & 4.5 \mathrm{E}-03\end{array}$

Production/Consumption factors:

Produce: $0.32 \quad$ Milk: $4.4 \quad$ Meat: 0.34

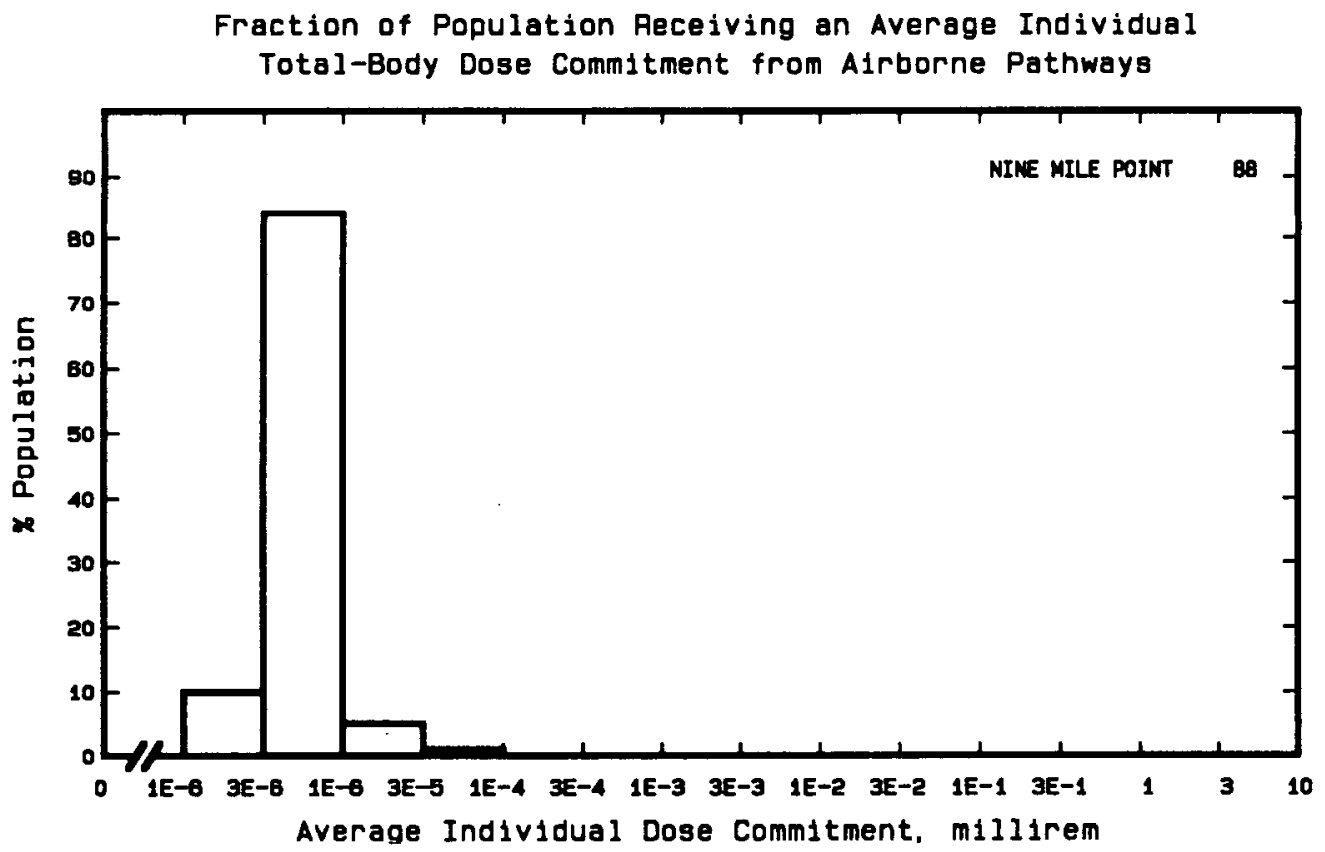


Site: NORTH ANNA

LOUISA COUNTY, VIRGINIA

Location: $\quad N 38.0608^{\circ} \quad$ W $77.7906^{\circ}$

POPULATION DATA

Total Population Within 2-to-80-km Region: 1.1E6

Major Metropolitan Centers Within Region:

Center

Richmond SMSA

Charlottesville

Fredricksburg

Culpeper

Ashl and
Population

680,000

44,000

16,000

7,000

5,000

\section{Location}

$66 \mathrm{~km} \quad$ SSE

$63 \mathrm{~km}$ W

$40 \mathrm{~km} \quad \mathrm{NE}$

$54 \mathrm{~km} \quad \mathrm{NNW}$

$41 \mathrm{~km} \quad \mathrm{SE}$

SITE-SPECIFIC DATA - AIRBORNE PATHWAYS

Average Annual State Production

of Crops and Animal Products

In $80-\mathrm{km}$ Radius Circle

Regional Productivity Factor:

Animal Grazing Factor:
Veg: $3.5 \mathrm{E} 7$ kilogram

Milk: $1.5 \mathrm{E} 8$ liter

Meat: $7.4 \mathrm{E} 7$ kilogram

0.9

0.7

Meteorology Period of Record: 1 APR 74 - 31 APR 75 Recovery: 99\%

SITE-SPECIFIC DATA - WATERBORNE PATHWAYS via LAKE ANNA(a)

Average Dilution Flow from

Plants: $3,500 \mathrm{ft}^{3} / \mathrm{s}$

Drinking Water:

None

Fish:

Edible Harvest: $7.3^{(\mathrm{b})} \mathrm{kg} / \mathrm{yr}$
Dilution Factor: $0.001(\mathrm{~b})$

(a) Reconcentration of radionuclides in lake accounted for (FES 1973).

(b) Average individual consumption rates as given in FES (1973) were used in lieu of catch data. 


\section{POPULATION DOSE-COMMITMENT ESTIMATES AND \\ AVERAGE INDIVIDUAL DOSE-COMMITMENT HISTOGRAM FOR}

NORTH ANNA 1 AND 2

Dose Commitments (person-rem) from Waterborne Pathways

Total Body

GI-LLI $\quad \underline{\text { Thyroid }}$

Bone

Liver

$\begin{array}{llllll}\text { Infant } & 0.0 \mathrm{E}+00 & 0.0 \mathrm{E}+00 & 0.0 \mathrm{E}+00 & 0.0 \mathrm{E}+00 & 0.0 \mathrm{E}+00 \\ \text { Child } & 4.6 \mathrm{E}-04 & 2.8 \mathrm{E}-04 & 1.2 \mathrm{E}-04 & 2.1 \mathrm{E}-03 & 2.4 \mathrm{E}-03 \\ \text { Teen } & 8.4 \mathrm{E}-04 & 5.3 \mathrm{E}-04 & 9.5 \mathrm{E}-05 & 1.3 \mathrm{E}-03 & 2.1 \mathrm{E}-03 \\ \text { Adult } & 8.9 \mathrm{E}-03 & 4.6 \mathrm{E}-03 & 6.8 \mathrm{E}-04 & 7.4 \mathrm{E}-03 & 1.2 \mathrm{E}-02 \\ \text { TOTAL } & 1.0 \mathrm{E}-02 & 5.4 \mathrm{E}-03 & 9.0 \mathrm{E}-04 & 1.1 \mathrm{E}-02 & 1.7 \mathrm{E}-02\end{array}$

Dose Commitments (person-rem) from Airborne Pathways

Total Body GI-LLI Thyroid Bone Liver lung

$\begin{array}{lllllll}\text { Infant } & 3.8 \mathrm{E}-04 & 3.7 \mathrm{E}-04 & 1.9 \mathrm{E}-03 & 1.3 \mathrm{E}-04 & 4.5 \mathrm{E}-04 & 3.8 \mathrm{E}-04 \\ \text { Child } & 5.0 \mathrm{E}-03 & 4.9 \mathrm{E}-03 & 1.3 \mathrm{E}-02 & 1.3 \mathrm{E}-03 & 5.5 \mathrm{E}-03 & 5.1 \mathrm{E}-03 \\ \text { Teen } & 3.2 \mathrm{E}-03 & 3.1 \mathrm{E}-03 & 6.3 \mathrm{E}-03 & 7.4 \mathrm{E}-04 & 3.3 \mathrm{E}-03 & 3.2 \mathrm{E}-03 \\ \text { Adult } & 1.8 \mathrm{E}-02 & 1.7 \mathrm{E}-02 & 2.7 \mathrm{E}-02 & 3.9 \mathrm{E}-03 & 1.8 \mathrm{E}-02 & 1.8 \mathrm{E}-02 \\ \text { TOTAL } & 2.6 \mathrm{E}-02 & 2.5 \mathrm{E}-02 & 4.9 \mathrm{E}-02 & 6.1 \mathrm{E}-03 & 2.7 \mathrm{E}-02 & 2.6 \mathrm{E}-02\end{array}$

Production/Consumption factors:

Produce: $0.14 \quad$ Milk: $0.90 \quad$ Meat: 0.72

Fraction of Population Receiving an Average Individual Total-Body Dose Commitment from Airborne Pathways

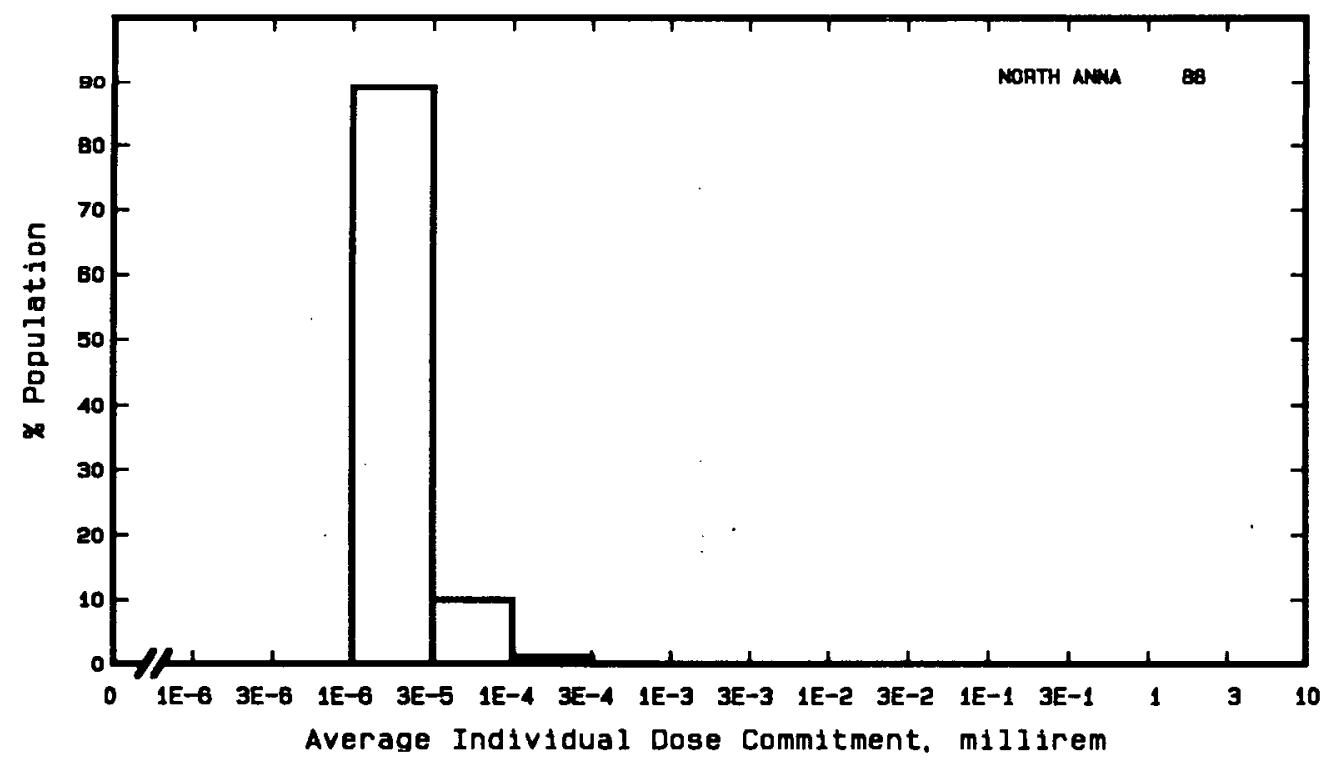


Site: OCONEE

OCONEE COUNTY, SOUTH CAROLINA

Location: $\quad N 34.7917^{\circ} \quad$ W $82.8986^{\circ}$

POPULATION DATA

Total Population Within 2-to-80-km Region: 9.9E5

Major Metropolitan Centers Within Region:

Center

Greenville SMSA

Anderson

Easley

Greer
Population

630,000

29,000

15,000

12,000

\section{Location}

$46 \mathrm{~km} \quad \mathrm{E}$

$39 \mathrm{~km} \quad \mathrm{SE}$

$27 \mathrm{~km} \quad \mathrm{E}$

$64 \mathrm{~km}$ ENE

\title{
SITE-SPECIFIC DATA - AIRBORNE PATHWAYS
}

Average Annual State Production

of Crops and Animal Products

In $80-\mathrm{km}$ Radius Circle

Regional Productivity Factor:

Animal Grazing Factor:
Veg: 7.4E6 kilogram

Milk: 5.7E7 liter

Meat: 5.0E7 kilogram

1

0.7

Meteorology Period of Record: 1 JAN 75 - 31 DEC 75 Recovery: $86 \%$

\section{RIVER}

\author{
Average River Flow \\ at Site: $1,100 \mathrm{ft}^{3} / \mathrm{s}$ \\ Drinking Water: Exposed Population: 59,000 \\ Dilution Factor: 1 \\ Fish: \\ Edible Harvest: (a) \\ Dilution Factor: $0.01^{(b)}$
}

(a) No fish catch data given in FES, so generic consumption rates used (Table A-1).

(b) Ten percent of population obtain 10\% of their fish diet from Hartwell Reservoir according to FES (1972). 


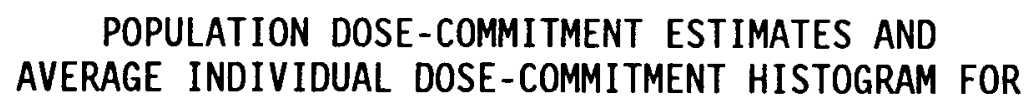

OCONEE 1,2 , AND 3

Dose Commitments (person-rem) from Waterborne Pathways

Total Body

GI-LII

Thyroid

Bone

Liver

Infant

3. 5E-02

4. $0 \mathrm{E}-02$

2.8E-01

1. $3 \mathrm{E}-02$

4. $6 \mathrm{E}-02$

Child

4.5E-01

$5.8 \mathrm{E}-01$

2. $1 \mathrm{E}+00$

4. $1 \mathrm{E}-01$

$7.8 \mathrm{E}-01$

Teen

2. $6 \mathrm{E}-01$

4. $2 \mathrm{E}-01$

6.7E-01

2. $0 \mathrm{E}-01$

4. $4 \mathrm{E}-01$

Adult

2. $5 \mathrm{E}+00$

4. $9 \mathrm{E}+00$

1. $2 \mathrm{E}+00$

$3.0 E+00$

TOTAL

3. $3 \mathrm{E}+00$

$4.8 \mathrm{E}+00$

$7.9 \mathrm{E}+00$

$1.8 \mathrm{E}+00$

$4.3 \mathrm{E}+00$

Dose Commitments (person-rem) from Airborne Pathways

Total Body GI-LII Thyroid Bone Liver Lung

$\begin{array}{lllllll}\text { Infant } & 7.7 \mathrm{E}-03 & 7.7 \mathrm{E}-03 & 3.4 \mathrm{E}-02 & 7.8 \mathrm{E}-03 & 7.9 \mathrm{E}-03 & 8.5 \mathrm{E}-03 \\ \text { Child } & 8.7 \mathrm{E}-02 & 8.6 \mathrm{E}-02 & 2.5 \mathrm{E}-01 & 8.7 \mathrm{E}-02 & 8.7 \mathrm{E}-02 & 9.8 \mathrm{E}-02 \\ \text { Teen } & 6.3 \mathrm{E}-02 & 6.3 \mathrm{E}-02 & 1.3 \mathrm{E}-01 & 6.2 \mathrm{E}-02 & 6.4 \mathrm{E}-02 & 7.8 \mathrm{E}-02 \\ \text { Adult } & 3.8 \mathrm{E}-01 & 3.8 \mathrm{E}-01 & 6.5 \mathrm{E}-01 & 3.7 \mathrm{E}-01 & 3.8 \mathrm{E}-01 & 4.3 \mathrm{E}-01 \\ \text { TOTAL } & 5.4 \mathrm{E}-01 & 5.4 \mathrm{E}-01 & 1.1 \mathrm{E}+00 & 5.3 \mathrm{E}-01 & 5.4 \mathrm{E}-01 & 6.1 \mathrm{E}-01\end{array}$

Production/Consumption factors:

Produce: 0.039 Milk: $0.44 \quad$ Meat: 0.63

Fraction of Population Receiving an Average Individual Total-Body Dose Commitment from Airborne Pathways

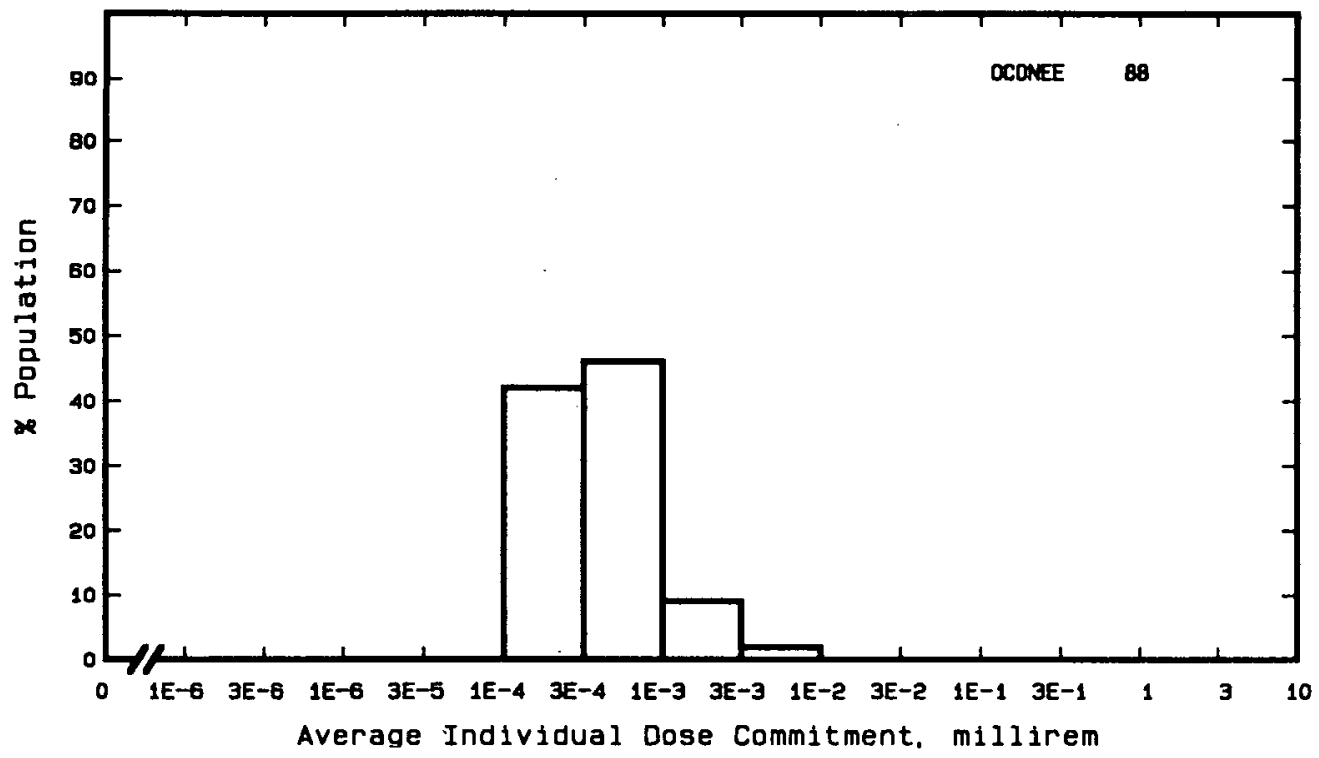


Site: OYSTER CREEK

OYSTER CREEK, NEW JERSEY

Location: $\quad \mathrm{N} 39.8142^{\circ}$ W $74.2064^{\circ}$

POPULATION DATA

Total Population Within 2-to-80-km Region: 3.6E6

Major Metropolitan Centers Within Region:

Center Population Location

New Brunswick-Sayreville SMSA

640,000

520,000

320,000

Trenton SMSA

190,000

$77 \mathrm{~km} \quad \mathrm{~N}$

$57 \mathrm{~km} \quad \mathrm{NNE}$

$66 \mathrm{~km} \quad \mathrm{NW}$

Atlantic City SMSA

88,000

$55 \mathrm{~km} \quad \mathrm{SSW}$

Camden

SITE-SPECIFIC DATA - AIRBORNE PATHWAYS

Average Annual State Production

of Crops and Animal Products

In $80-\mathrm{km}$ Radius Circle

Regional Productivity Factor:

Animal Grazing Factor:
Veg: $7.4 E 7$ kilogram

Milk: 2.7E8 liter

Meat: $2.4 \mathrm{E} 7$ kilogram

0.5

0.6

Meteorology Period of Record: 15 FEB 66 - 31 DEC 68 Recovery: $63 \%$

SITE-SPECIFIC DATA - WATERBORNE PATHWAYS via BARNEGAT BAY

Average Dilution Flow

from Plant: $26 \mathrm{ft}^{3} / \mathrm{s}$

Fish:

Edible Harvest: 2.1 (a) $\mathrm{kg} / \mathrm{yr}$

Invertebrates:

Edible Harvest: 0.96 (a) $\mathrm{kg} / \mathrm{yr}$

Dilution Factor: $0.01^{\text {(b) }}$

(a) Average individual consumption rate as given in the FES (1974) used in lieu of catch data.

(b) $10 \%$ of seafood eaten assumed caught in bay waters diluted to $10 \%$ of that of discharge canal according to FES. 
POPULATION DOSE-COMMITMENT ESTIMATES AND

AVERAGE INDIVIDUAL DOSE-COMMITMENT HISTOGRAM FOR

OYSTER CREEK

Dose Commitments (person-rem) from Waterborne Pathways

Total Body GI-LII $\underline{\text { Thyroid }}$ Bone Liver

$\begin{array}{llllll}\text { Infant } & 0.0 \mathrm{E}+00 & 0.0 \mathrm{E}+00 & 0.0 \mathrm{E}+00 & 0.0 \mathrm{E}+00 & 0.0 \mathrm{E}+00 \\ \text { Child } & 2.7 \mathrm{E}-02 & 4.6 \mathrm{E}-02 & 1.9 \mathrm{E}-03 & 1.7 \mathrm{E}-02 & 2.4 \mathrm{E}-02 \\ \text { Teen } & 2.1 \mathrm{E}-02 & 9.5 \mathrm{E}-02 & 1.5 \mathrm{E}-03 & 1.0 \mathrm{E}-02 & 2.0 \mathrm{E}-02 \\ \text { Adult } & 1.5 \mathrm{E}-01 & 8.4 \mathrm{E}-01 & 1.1 \mathrm{E}-02 & 5.9 \mathrm{E}-02 & 1.2 \mathrm{E}-01 \\ \text { TOTAL } & 2.0 \mathrm{E}-01 & 9.8 \mathrm{E}-01 & 1.4 \mathrm{E}-02 & 8.6 \mathrm{E}-02 & 1.7 \mathrm{E}-01\end{array}$

Dose Commitments (person-rem) from Airborne Pathways

Total Body GI-LII Ihyroid Bone Liver Lung

$\begin{array}{lllllll}\text { Infant } & 2.9 \mathrm{E}-02 & 2.9 \mathrm{E}-02 & 6.1 \mathrm{E}-02 & 2.9 \mathrm{E}-02 & 2.9 \mathrm{E}-02 & 3.0 \mathrm{E}-02 \\ \text { Child } & 3.2 \mathrm{E}-01 & 3.2 \mathrm{E}-01 & 5.5 \mathrm{E}-01 & 3.2 \mathrm{E}-01 & 3.2 \mathrm{E}-01 & 3.3 \mathrm{E}-01 \\ \text { Teen } & 2.4 \mathrm{E}-01 & 2.4 \mathrm{E}-01 & 3.5 \mathrm{E}-01 & 2.3 \mathrm{E}-01 & 2.4 \mathrm{E}-01 & 2.5 \mathrm{E}-01 \\ \text { Adult } & 1.4 \mathrm{E}+00 & 1.4 \mathrm{E}+00 & 1.9 \mathrm{E}+00 & 1.4 \mathrm{E}+00 & 1.4 \mathrm{E}+00 & 1.5 \mathrm{E}+00 \\ \text { TOTAL } & 2.0 \mathrm{E}+00 & 2.0 \mathrm{E}+00 & 2.8 \mathrm{E}+00 & 2.0 \mathrm{E}+00 & 2.0 \mathrm{E}+00 & 2.1 \mathrm{E}+00\end{array}$

Production/Consumption factors:

Produce: $0.052 \quad$ Milk: $0.28 \quad$ Meat: 0.041

Fraction of Population Receiving an Average Individual Total-Body Dose Commitment from Airborne Pathways

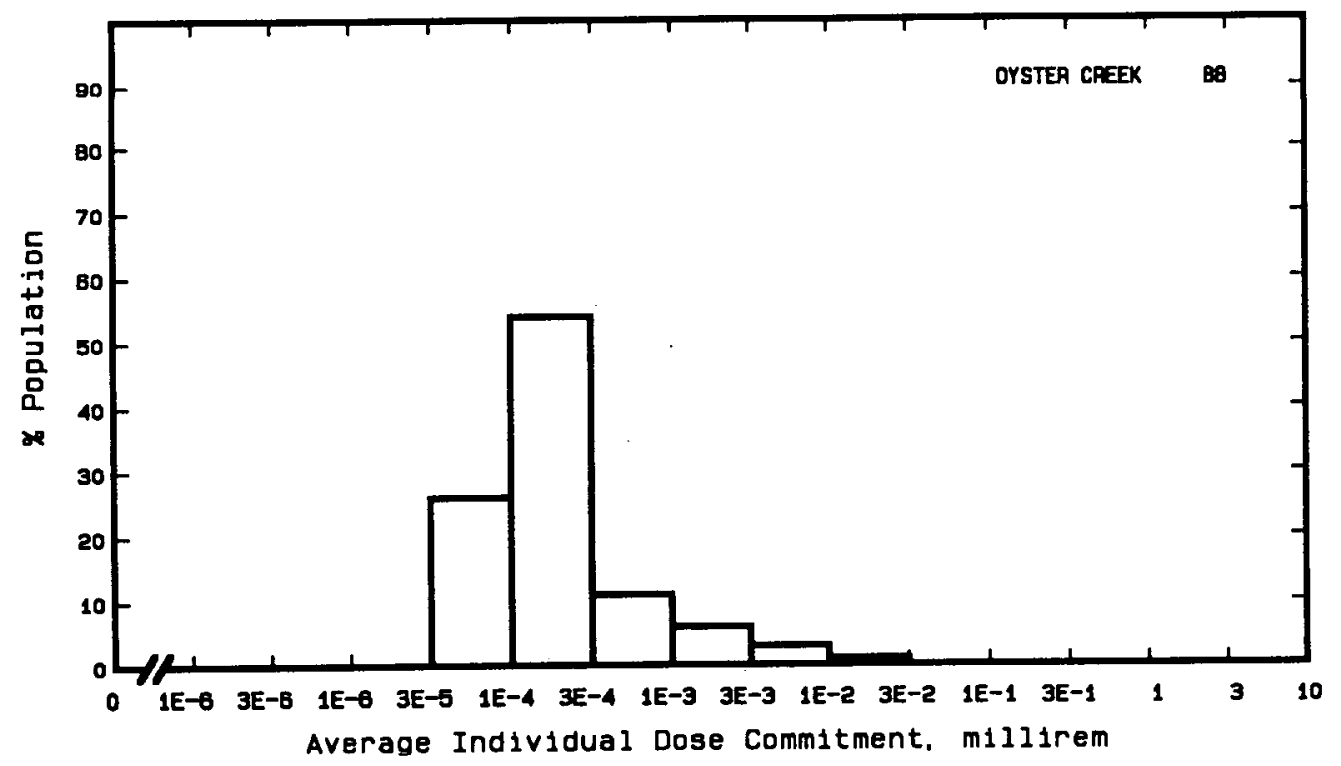


Site: PALISADES

COVERT TOWNSHIP, MICHIGAN

Location: $\quad$ N $42.3222^{\circ}$ W $86.3153^{\circ}$

POPULATION DATA

Total Population Within 2-to-80-km Region: 1.1 E6

Major Metropolitan Centers Within Region:

\section{Center}

Kal amazoo-Portage SMSA South Bend SMSA

Elkhart SMSA

Holl and

Benton Harbor
Population

280,000

280,000

140,000

26,000

15,000

\section{Location}

$61 \mathrm{~km} \quad \mathrm{E}$

$72 \mathrm{~km} \mathrm{~S}$

$76 \mathrm{~km} \quad \mathrm{SSE}$

$53 \mathrm{~km} \quad \mathrm{NNE}$

$25 \mathrm{~km}$ SSW

\section{SITE-SPECIFIC DATA - AIRBORNE PATHWAYS}
Average Annual State Production
Veg: $6.8 \mathrm{E} 7 \mathrm{ki}$ logram
of Crops and Animal Products
Milk: 2.9E8 liter
In $80-\mathrm{km}$ Radius Circle
Meat: $4.5 \mathrm{E7} \mathrm{kilogram}$

Regional Productivity Factor:

0.6

Animal Grazing Factor:

0.5

Meteorology Period of Record: 1 SEP 73 - 31 AUG 74 Recovery: 67\%

\section{SITE-SPECIFIC DATA - WATERBORNE PATHWAYS via LAKE MICHIGAN}

\section{Average Dilution Flow from} Plant: $130 \mathrm{ft}^{3} / \mathrm{s}$

Drinking Water:

Exposed Population: 51,000

Dilution Factor: $3.5 \mathrm{E}-3(\mathrm{a})$

Fish:

Edible Harvest: (b)

Dilution Factor: 0.001

(a) Drinking water dilution factor est imated by averaging dilution factor derived from FES (1972) suitably weighted for population.

(b) Average individual consumption rate of $20 \mathrm{~g} / \mathrm{d}(7.3 \mathrm{~kg} / \mathrm{yr})$ as given in FES used in lieu of catch data. 
POPULATION DOSE-COMMITMENT ESTIMATES AND

AVERAGE INDIVIDUAL DOSE-COMMITMENT HISTOGRAM FOR

PALISADES

Dose Commitments (person-rem) from Waterborne Pathways

Total Body GI-LII Ihyroid Bone Liver

$\begin{array}{llllll}\text { Infant } & 3.6 \mathrm{E}-04 & 3.4 \mathrm{E}-04 & 3.6 \mathrm{E}-04 & 1.2 \mathrm{E}-04 & 3.9 \mathrm{E}-04 \\ \text { Child } & 1.1 \mathrm{E}-02 & 4.4 \mathrm{E}-03 & 4.2 \mathrm{E}-03 & 5.0 \mathrm{E}-02 & 5.0 \mathrm{E}-02 \\ \text { Teen } & 1.5 \mathrm{E}-02 & 2.5 \mathrm{E}-03 & 1.7 \mathrm{E}-03 & 2.9 \mathrm{E}-02 & 3.9 \mathrm{E}-02 \\ \text { Adult } & 1.6 \mathrm{E}-01 & 2.1 \mathrm{E}-02 & 1.4 \mathrm{E}-02 & 1.7 \mathrm{E}-01 & 2.3 \mathrm{E}-01 \\ \text { TOTAL } & 1.9 \mathrm{E}-01 & 2.8 \mathrm{E}-02 & 2.1 \mathrm{E}-02 & 2.5 \mathrm{E}-01 & 3.2 \mathrm{E}-01\end{array}$

Dose Commitments (person-rem) from Airborne Pathways

Total Body GI-LII Ihyroid Bone Liver Lung

$\begin{array}{lllllll}\text { Infant } & 3.8 \mathrm{E}-04 & 3.6 \mathrm{E}-04 & 9.3 \mathrm{E}-03 & 3.8 \mathrm{E}-04 & 3.9 \mathrm{E}-04 & 4.0 \mathrm{E}-04 \\ \text { Child } & 4.2 \mathrm{E}-03 & 4.1 \mathrm{E}-03 & 5.7 \mathrm{E}-02 & 4.1 \mathrm{E}-03 & 4.2 \mathrm{E}-03 & 4.5 \mathrm{E}-03 \\ \text { Teen } & 3.0 \mathrm{E}-03 & 3.0 \mathrm{E}-03 & 2.4 \mathrm{E}-02 & 2.9 \mathrm{E}-03 & 3.0 \mathrm{E}-03 & 3.6 \mathrm{E}-03 \\ \text { Adult } & 1.8 \mathrm{E}-02 & 1.8 \mathrm{E}-02 & 8.5 \mathrm{E}-02 & 1.7 \mathrm{E}-02 & 1.8 \mathrm{E}-02 & 2.0 \mathrm{E}-02 \\ \text { TOTAL } & 2.5 \mathrm{E}-02 & 2.5 \mathrm{E}-02 & 1.8 \mathrm{E}-01 & 2.5 \mathrm{E}-02 & 2.6 \mathrm{E}-02 & 2.8 \mathrm{E}-02\end{array}$

Production/Consumption factors:

Produce: 0.20 Milk: 1.2 Meat: 0.32
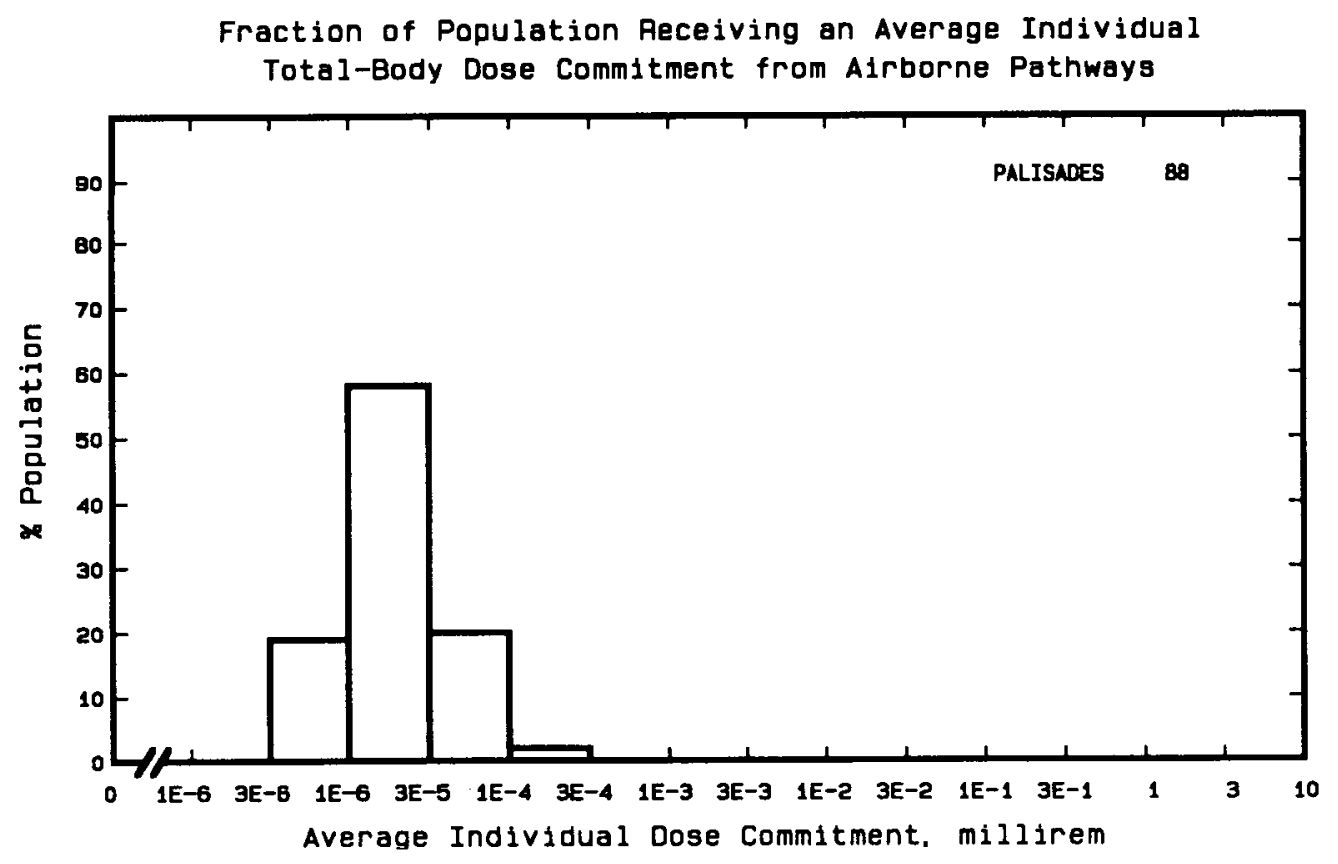
Site: PALO VERDE

WINTERSBURG, ARIZONA

Location: $\quad$ N $33.4200^{\circ} \quad$ W $112.8683^{\circ}$

POPULATION DATA

Total Population Within 2-to-80-km Region: 1.2E6

Major Metropolitan Centers Within Region:

Center

Phoenix MSA

Avondale
Population

$1,900,000$

8,200

\section{Location}

$64 \mathrm{~km} \quad \mathrm{E}$

$49 \mathrm{~km} \quad \mathrm{E}$

SITE-SPECIFIC DATA - AIRBORNE PATHWAYS

Average Annual State Production

Veg: $1.2 \mathrm{E} 7 \mathrm{kilogram}$

of Crops and Animal Products

In $80-\mathrm{km}$ Radius Circle

Milk: 2.3E7 liter

Meat: $2.1 E 7$ kilogram

Regional Productivity Factor:

Animal Grazing Factor:

0.9

$0.1 \operatorname{Milk}(a)$

0.9 Beef

Meteorology Period of Record: 13 AUG $73-8$ AUG 78 Recovery: 93\%

SITE-SPECIFIC DATA - WATERBORNE PATHWAYS None

Average River Flow

at Site: (b)

Drinking Water: None (b)

Fish:

None (b)

(a) Milk cows fed from dry lot $90 \%$ of time (FES 1975).

(b) No waterborne pathways 
POPULATION DOSE-COMMITMENT ESTIMATES AND

AVERAGE INDIVIDUAL DOSE-COMMITMENT HISTOGRAM FOR

PALO VERDE 1, 2, AND 3

Dose Commitments (person-rem) from Waterborne Pathways

Infant

Total Body GI-LLI $\underline{\text { Thyroid }}$ Bone Liver

Child

Teen

Adult

(No Waterborne Pathway Doses) (a)

TOTAL

Dose Commitments (person-rem) from Airborne Pathways

Total Body GI-LLI Thyroid Bone Liver Lung

$\begin{array}{lllllll}\text { Infant } & 2.0 \mathrm{E}-03 & 2.0 \mathrm{E}-03 & 3.5 \mathrm{E}-03 & 7.3 \mathrm{E}-04 & 2.0 \mathrm{E}-03 & 2.1 \mathrm{E}-03 \\ \text { Child } & 3.2 \mathrm{E}-02 & 3.2 \mathrm{E}-02 & 5.3 \mathrm{E}-02 & 8.1 \mathrm{E}-03 & 3.2 \mathrm{E}-02 & 3.3 \mathrm{E}-02 \\ \text { Teen } & 2.3 \mathrm{E}-02 & 2.3 \mathrm{E}-02 & 3.4 \mathrm{E}-02 & 5.9 \mathrm{E}-03 & 2.3 \mathrm{E}-02 & 2.5 \mathrm{E}-02 \\ \text { Adult } & 1.4 \mathrm{E}-01 & 1.4 \mathrm{E}-01 & 1.9 \mathrm{E}-01 & 3.6 \mathrm{E}-02 & 1.4 \mathrm{E}-01 & 1.4 \mathrm{E}-01 \\ \text { TOTAL } & 2.0 \mathrm{E}-01 & 2.0 \mathrm{E}-01 & 2.8 \mathrm{E}-01 & 5.1 \mathrm{E}-02 & 2.0 \mathrm{E}-01 & 2.0 \mathrm{E}-01\end{array}$

Production/Consumption factors:

Produce: 0.045 Milk: 0.13 Meat: 0.20

Fraction of Population Receiving an Average Individual

Total-Body Dose Commitment from Alrborne Pathwayg

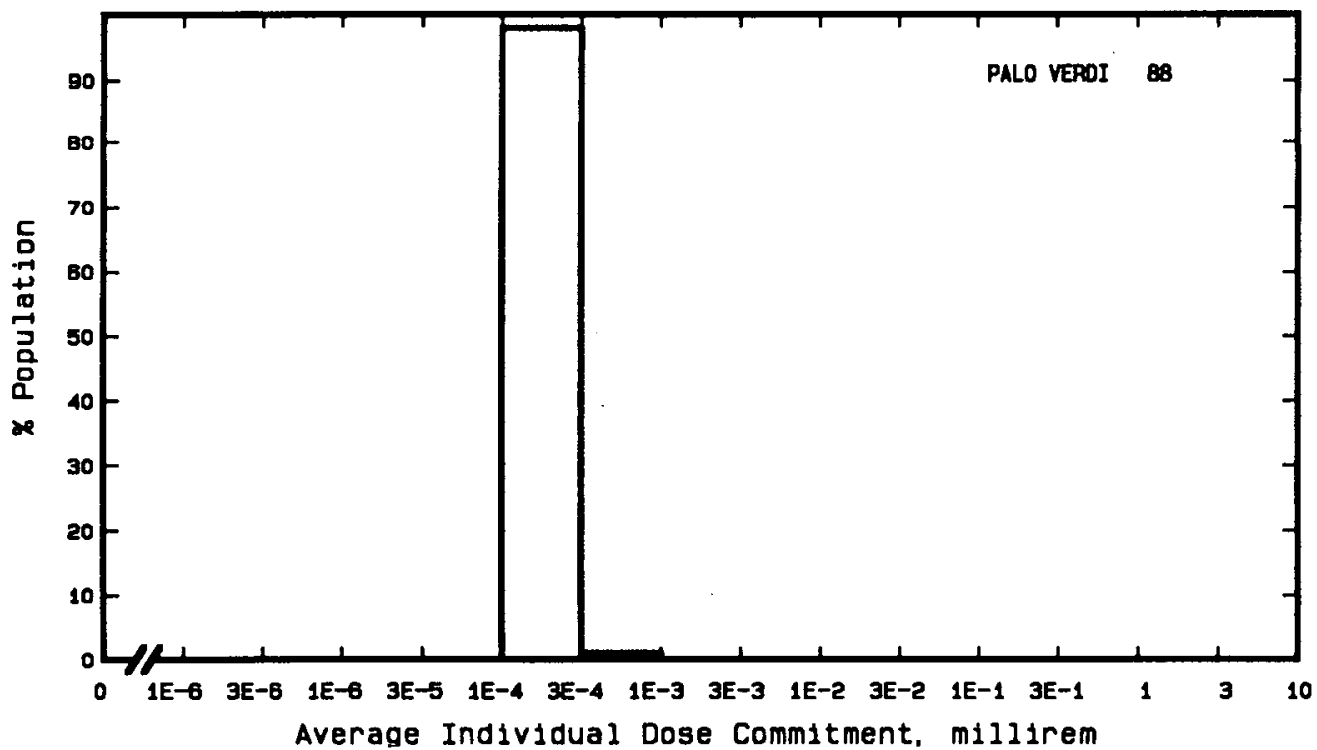

(a) No liquid releases reported (Tichler, et al. 1991) 
Site: PEACH BOTTOM

YORK COUNTY, PENNSYLVANIA

Location: $\quad$ N $39.7589^{\circ}$ W $76.2692^{\circ}$

POPULATION DATA

Total Population Within 2-to-80-km Region: 4.3E6

Major Metropolitan Centers Within Region:

Center

Baltimore SMSA

Harrisburg SMSA

Wilmington SMSA

York SMSA

Lancaster SMSA
Population

$2,200,000$

450,000

400,000

380,000

360,000
Location

$60 \mathrm{~km} \quad \mathrm{SSW}$

$77 \mathrm{~km} \quad \mathrm{NNW}$

$62 \mathrm{~km} \quad \mathrm{E}$

$45 \mathrm{~km} \quad \mathrm{NW}$

$31 \mathrm{~km} \mathrm{~N}$

SITE-SPECIFIC DATA - AIRBORNE PATHWAYS

Average Annual State Production

of Crops and Animal Products

In $80-\mathrm{km}$ Radius Circle

Regional Productivity Factor:

Animal Grazing Factor:
Veg: $5.3 \mathrm{E} 7$ kilogram

Milk: 5.3E8 liter

Meat: $5.4 \mathrm{E} 7 \mathrm{ki}$ logram

0.95

0.6

Meteorology Period of Record: I AUG 67 - 31 JUL 71 Recovery: 72\%

SITE-SPECIFIC DATA - WATERBORNE PATHWAYS via SUSQUEHANNA RIVER

Average River Flow

at Site: $36,000 \mathrm{ft}^{3} / \mathrm{s}$

Drinking Water:

Exposed Population: 2.2E6

Dilution Factor: 1

Fish:

Edible Harvest: (a)

Dilution Factor: $0.001^{(b)}$

(a) No fish catch data given in FES (1974), thus generic consumption rates used (Table A-1).

(b) One percent of people obta in $10 \%$ of their fish diet from river down-stream from plant according to FES. 
POPULATION DOSE-COMMITMENT ESTIMATES AND

AVERAGE INDIVIDUAL DOSE-COMMITMENT HISTOGRAM FOR

PEACH BOTTOM 2 AND 3

Dose Commitments (person-rem) from Waterborne Pathways

Total. Body GI-LII $\underline{\text { Thyroid }}$ Bone Liver

$\begin{array}{llllll}\text { Infant } & 2.3 \mathrm{E}-03 & 1.0 \mathrm{E}-03 & 5.1 \mathrm{E}-04 & 1.2 \mathrm{E}-02 & 1.4 \mathrm{E}-02 \\ \text { Child } & 3.7 \mathrm{E}-02 & 1.0 \mathrm{E}-02 & 5.7 \mathrm{E}-03 & 1.4 \mathrm{E}-01 & 1.3 \mathrm{E}-01 \\ \text { Teen } & 2.4 \mathrm{E}-02 & 6.0 \mathrm{E}-03 & 2.2 \mathrm{E}-03 & 3.8 \mathrm{E}-02 & 4.9 \mathrm{E}-02 \\ \text { Adult } & 2.5 \mathrm{E}-01 & 5.4 \mathrm{E}-02 & 1.8 \mathrm{E}-02 & 2.6 \mathrm{E}-01 & 3.2 \mathrm{E}-01 \\ \text { TOTAL } & 3.2 \mathrm{E}-01 & 7.1 \mathrm{E}-02 & 2.7 \mathrm{E}-02 & 4.4 \mathrm{E}-01 & 5.1 \mathrm{E}-01\end{array}$

Dose Commitments (person-rem) from Airborne Pathways

Total Body GI-LII Thyroid Bone Liver Lung

$\begin{array}{lllllll}\text { Infant } & 2.5 \mathrm{E}-04 & 2.5 \mathrm{E}-04 & 2.2 \mathrm{E}-04 & 1.1 \mathrm{E}-04 & 3.0 \mathrm{E}-04 & 2.6 \mathrm{E}-04 \\ \text { Child } & 3.0 \mathrm{E}-03 & 2.7 \mathrm{E}-03 & 2.6 \mathrm{E}-03 & 1.7 \mathrm{E}-03 & 3.1 \mathrm{E}-03 & 3.3 \mathrm{E}-03 \\ \text { Teen } & 1.8 \mathrm{E}-03 & 1.7 \mathrm{E}-03 & 1.6 \mathrm{E}-03 & 9.4 \mathrm{E}-04 & 1.9 \mathrm{E}-03 & 2.2 \mathrm{E}-03 \\ \text { Adult } & 9.3 \mathrm{E}-03 & 9.0 \mathrm{E}-03 & 8.5 \mathrm{E}-03 & 4.6 \mathrm{E}-03 & 9.2 \mathrm{E}-03 & 1.1 \mathrm{E}-02 \\ \text { TOTAL } & 1.4 \mathrm{E}-02 & 1.4 \mathrm{E}-02 & 1.3 \mathrm{E}-02 & 7.3 \mathrm{E}-03 & 1.4 \mathrm{E}-02 & 1.7 \mathrm{E}-02\end{array}$

Production/Consumption factors:

Produce: $0.060 \quad$ Milk: $0.88 \quad$ Meat: 0.15

Fraction of Population Receiving an Average Individual

Total-Body Dose Commitment from Airborne Pathways

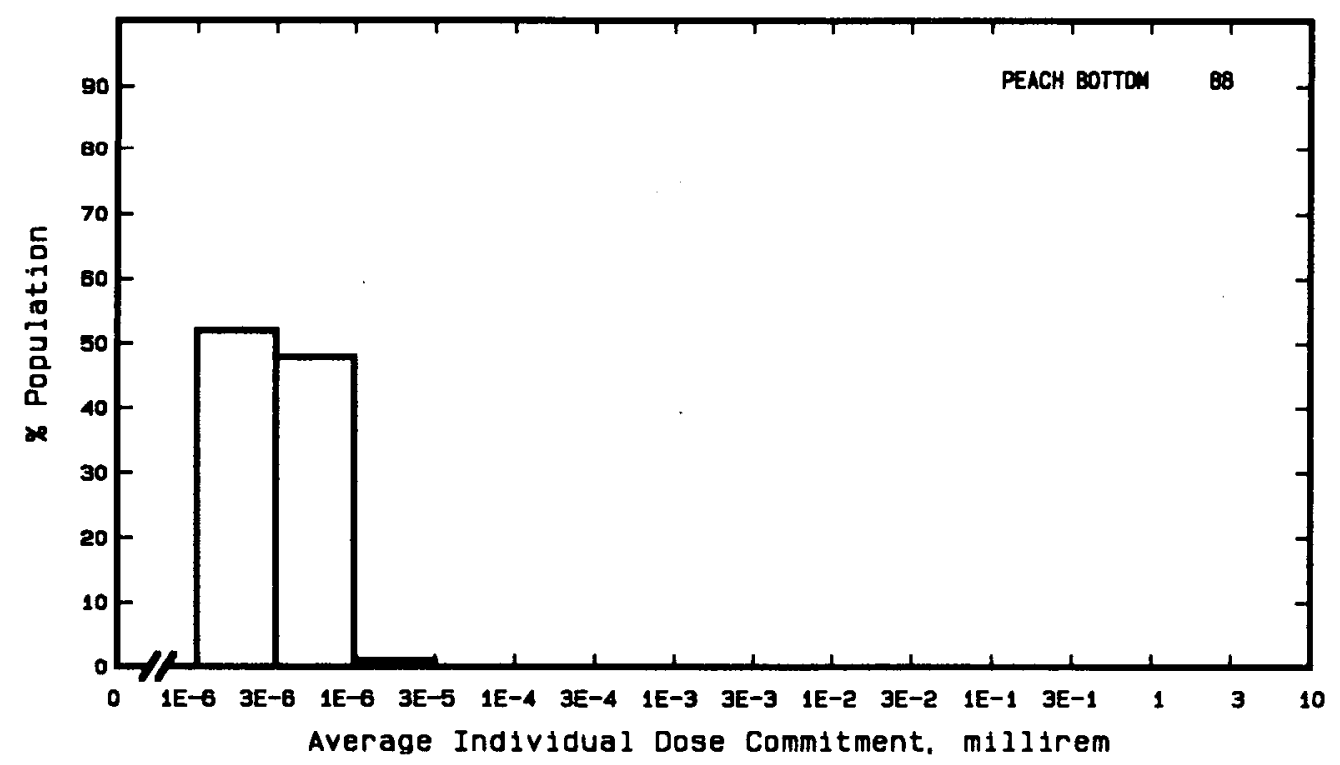


Site: PERRY

NORTH PERRY, OHIO

Location: $\quad N 41.8008^{\circ} \quad$ W $81.1433^{\circ}$

POPULATION DATA

Total Population Within 2-to-80-km Region: 2.4E6

Major Metropolitan Centers Within Region:

\begin{tabular}{|c|c|c|c|}
\hline Center & Population & \multicolumn{2}{|c|}{ Location } \\
\hline $\begin{array}{l}\text { Cleveland SMS } \\
\text { Akron } \\
\text { Warren } \\
\text { Ashtabula } \\
\text { Painesville City }\end{array}$ & $\begin{array}{r}580,000 \\
250,000 \\
54,000 \\
24,000 \\
16,000\end{array}$ & $\begin{array}{ll}53 & \mathrm{~km} \\
80 & \mathrm{~km} \\
70 & \mathrm{~km} \\
35 & \mathrm{~km} \\
11 & \mathrm{~km}\end{array}$ & $\begin{array}{l}\text { SW } \\
\text { SSW } \\
\text { SE } \\
N E \\
\text { SW }\end{array}$ \\
\hline
\end{tabular}

SITE-SPECIFIC DATA - AIRBORNE PATHWAYS

Average Annual State Production

of Crops and Animal Products

In $80-\mathrm{km}$ Radius Circle

Regional Productivity Factor:

Animal Grazing Factor:
Veg: $6.9 E 7$ kilogram

Milk: 3.7 E6 liter

Meat: 1.2E8 kilogram

0.45

0.5

Meteorology Period of Record: 1 MAY 72 - 31 AUG 78 Recovery: 92\%

SITE-SPECIFIC DATA - WATERBORNE PATHWAYS via LAKE ERIE

Average Dilution Flow

from Plant: $160 \mathrm{ft}^{3} / \mathrm{s}$

Drinking Water:

Exposed Population: 1,700,000

Fish:

Edible Harvest: $1.2 \mathrm{E} 6 \mathrm{~kg} / \mathrm{yr}$

Dilution Factor: 0.016

(a) Est imated. 
POPULATION DOSE-COMMITMENT ESTIMATES AND

AVERAGE INDIVIDUAL DOSE-COMMITMENT HISTOGRAM FOR

PERRY

Dose Commitments (person-rem) from Waterborne Pathways

Total Body GI-LII Thyroid Bone Liver

$\begin{array}{llllll}\text { Infant } & 2.3 \mathrm{E}-03 & 2.3 \mathrm{E}-03 & 3.5 \mathrm{E}-02 & 3.0 \mathrm{E}-03 & 2.3 \mathrm{E}-03 \\ \text { Child } & 2.6 \mathrm{E}-02 & 3.4 \mathrm{E}-02 & 2.5 \mathrm{E}-01 & 3.3 \mathrm{E}-02 & 2.5 \mathrm{E}-02 \\ \text { Teen } & 9.6 \mathrm{E}-03 & 2.5 \mathrm{E}-02 & 7.4 \mathrm{E}-02 & 9.8 \mathrm{E}-03 & 1.0 \mathrm{E}-02 \\ \text { Adult } & 7.6 \mathrm{E}-02 & 2.3 \mathrm{E}-01 & 5.2 \mathrm{E}-01 & 6.6 \mathrm{E}-02 & 7.8 \mathrm{E}-02 \\ \text { TOTAL } & 1.1 \mathrm{E}-01 & 2.9 \mathrm{E}-01 & 8.7 \mathrm{E}-01 & 1.1 \mathrm{E}-01 & 1.1 \mathrm{E}-01\end{array}$

Dose Commitments (person-rem) from Airborne Pathways

Total Body GI-LII Thyroid Bone Liver Lung

$\begin{array}{lllllll}\text { Infant } & 9.4 \mathrm{E}-04 & 9.4 \mathrm{E}-04 & 4.3 \mathrm{E}-03 & 9.6 \mathrm{E}-04 & 9.5 \mathrm{E}-04 & 1.0 \mathrm{E}-03 \\ \text { Child } & 1.1 \mathrm{E}-02 & 1.1 \mathrm{E}-02 & 7.2 \mathrm{E}-02 & 1.2 \mathrm{E}-02 & 1.1 \mathrm{E}-02 & 1.1 \mathrm{E}-02 \\ \text { Teen } & 7.9 \mathrm{E}-03 & 7.8 \mathrm{E}-03 & 4.1 \mathrm{E}-02 & 8.4 \mathrm{E}-03 & 7.9 \mathrm{E}-03 & 8.8 \mathrm{E}-03 \\ \text { Adult } & 4.8 \mathrm{E}-02 & 4.7 \mathrm{E}-02 & 2.0 \mathrm{E}-01 & 5.0 \mathrm{E}-02 & 4.7 \mathrm{E}-02 & 5.0 \mathrm{E}-02 \\ \text { TOTAL } & 6.8 \mathrm{E}-02 & 6.7 \mathrm{E}-02 & 3.2 \mathrm{E}-01 & 7.2 \mathrm{E}-02 & 6.7 \mathrm{E}-02 & 7.2 \mathrm{E}-02\end{array}$

Production/Consumption factors:

Produce: 0.067 Milk: 0.0054 Meat: 0.27

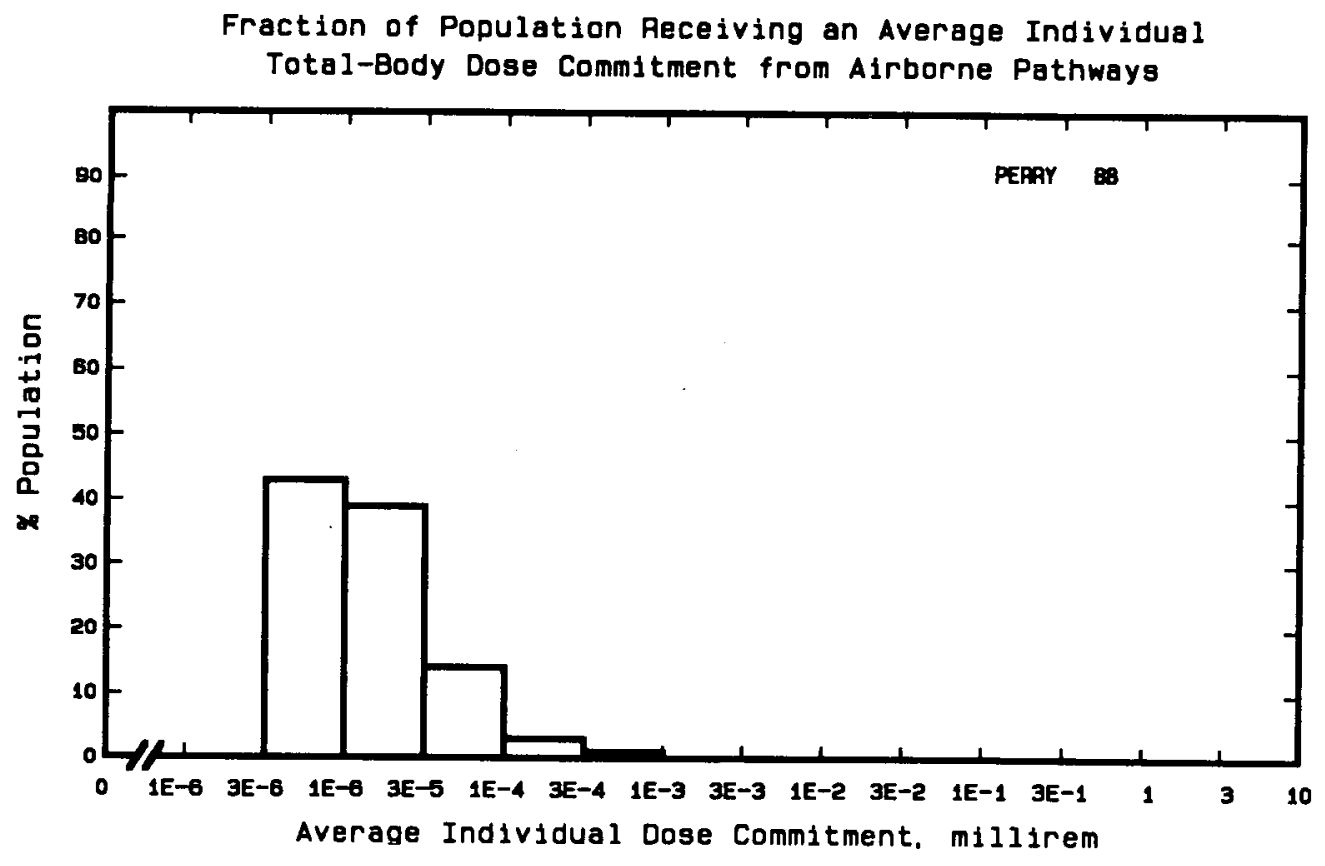


Site: PILGRIM

PLYMOUTH, MASSACHUSETTS

Location: $\quad$ N $41.9444^{\circ}$ W $70.5794^{\circ}$

POPULATION DATA

Total Population Within 2-to-80-km Region: 4.4E6

Major Metropolitan Centers Within Region:

Center

Population Location

Boston SMSA $2,800,000$

840,000

Providence-Warwick-Pawtucket SMSA

170,000

170,000

Brockton SMSA

150,000

$61 \mathrm{~km} \quad \mathrm{NW}$

$70 \mathrm{~km} \quad \mathrm{~W}$

$45 \mathrm{~km} \quad \mathrm{SSW}$

$40 \mathrm{~km} \quad W N W$

Fall River SMSA

Veg: $2.0 \mathrm{E} 7 \mathrm{kilogram}$

Milk: 2.6E8 liter

Meat: 1.6E7 kilogram

of Crops and Animal Products

In 80-km Radius Circle

0.3

Regional Productivity Factor:

0.6

Animal Grazing Factor:

$55 \mathrm{~km} \quad \mathrm{SW}$

Meteorology Period of Record: 1 MAY 74 - 30 APR 75 Recovery: $93 \%$

SITE-SPECIFIC DATA - WATERBORNE PATHWAYS via CAPE COD BAY

Average Dilution Flow

from Plant: $6.0 \mathrm{ft}^{3} / \mathrm{s}$

Fish:

Edible Harvest: $2.6 \mathrm{E} 4 \mathrm{~kg} / \mathrm{yr}$

Dilution Factor: 0.001

Invertebrates:

Edible Harvest: $3.1 \mathrm{E} 4 \mathrm{~kg} / \mathrm{yr}$

Dilution Factor: 0.002 
POPULATION DOSE-COMMITMENT ESTIMATES AND

AVERAGE INDIVIDUAL DOSE-COMMITMENT HISTOGRAM FOR

PILGRIM

Dose Commitments (person-rem) from Waterborne Pathways

Total Body GI-LII $\underline{\text { Thyroid }}$ Bone Liver

$\begin{array}{llllll}\text { Infant } & 0.0 \mathrm{E}+00 & 0.0 \mathrm{E}+00 & 0.0 \mathrm{E}+00 & 0.0 \mathrm{E}+00 & 0.0 \mathrm{E}+00 \\ \text { Child } & 1.9 \mathrm{E}-04 & 2.3 \mathrm{E}-04 & 1.1 \mathrm{E}-07 & 5.5 \mathrm{E}-04 & 4.0 \mathrm{E}-04 \\ \text { Teen } & 1.5 \mathrm{E}-04 & 4.7 \mathrm{E}-04 & 9.4 \mathrm{E}-08 & 3.1 \mathrm{E}-04 & 3.1 \mathrm{E}-04 \\ \text { Adult } & 1.1 \mathrm{E}-03 & 4.1 \mathrm{E}-03 & 7.5 \mathrm{E}-07 & 1.8 \mathrm{E}-03 & 1.8 \mathrm{E}-03 \\ \text { TOTAL } & 1.4 \mathrm{E}-03 & 4.8 \mathrm{E}-03 & 9.6 \mathrm{E}-07 & 2.7 \mathrm{E}-03 & 2.5 \mathrm{E}-03\end{array}$

Dose Commitments (person-rem) from Airborne Pathways

Total Body GI-LII Thyroid Bone Liver Lung

$\begin{array}{lllllll}\text { Infant } & 8.5 \mathrm{E}-06 & \mathbf{8 . 3 \mathrm { E } - 0 6} & 8.2 \mathrm{E}-06 & 9.8 \mathrm{E}-06 & 1.1 \mathrm{E}-05 & 1.4 \mathrm{E}-05 \\ \text { Child } & 9.8 \mathrm{E}-05 & 9.8 \mathrm{E}-05 & 9.5 \mathrm{E}-05 & 1.0 \mathrm{E}-04 & 1.1 \mathrm{E}-04 & 2.0 \mathrm{E}-04 \\ \text { Teen } & 7.3 \mathrm{E}-05 & 7.4 \mathrm{E}-05 & 7.0 \mathrm{E}-05 & 6.7 \mathrm{E}-05 & 7.8 \mathrm{E}-05 & 1.6 \mathrm{E}-04 \\ \text { Adult } & 4.4 \mathrm{E}-04 & 4.5 \mathrm{E}-04 & 4.2 \mathrm{E}-04 & 3.9 \mathrm{E}-04 & 4.4 \mathrm{E}-04 & 8.0 \mathrm{E}-04 \\ \text { TOTAL } & 6.2 \mathrm{E}-04 & 6.3 \mathrm{E}-04 & 5.9 \mathrm{E}-04 & 5.7 \mathrm{E}-04 & 6.4 \mathrm{E}-04 & 1.2 \mathrm{E}-03\end{array}$

Production/Consumption factors:

Produce: $0.0070 \quad$ Milk: $0.13 \quad$ Meat: 0.014

Fraction of Population Receiving an Average Individual

Total-Body Dose Commitment from Alrborne Pathways

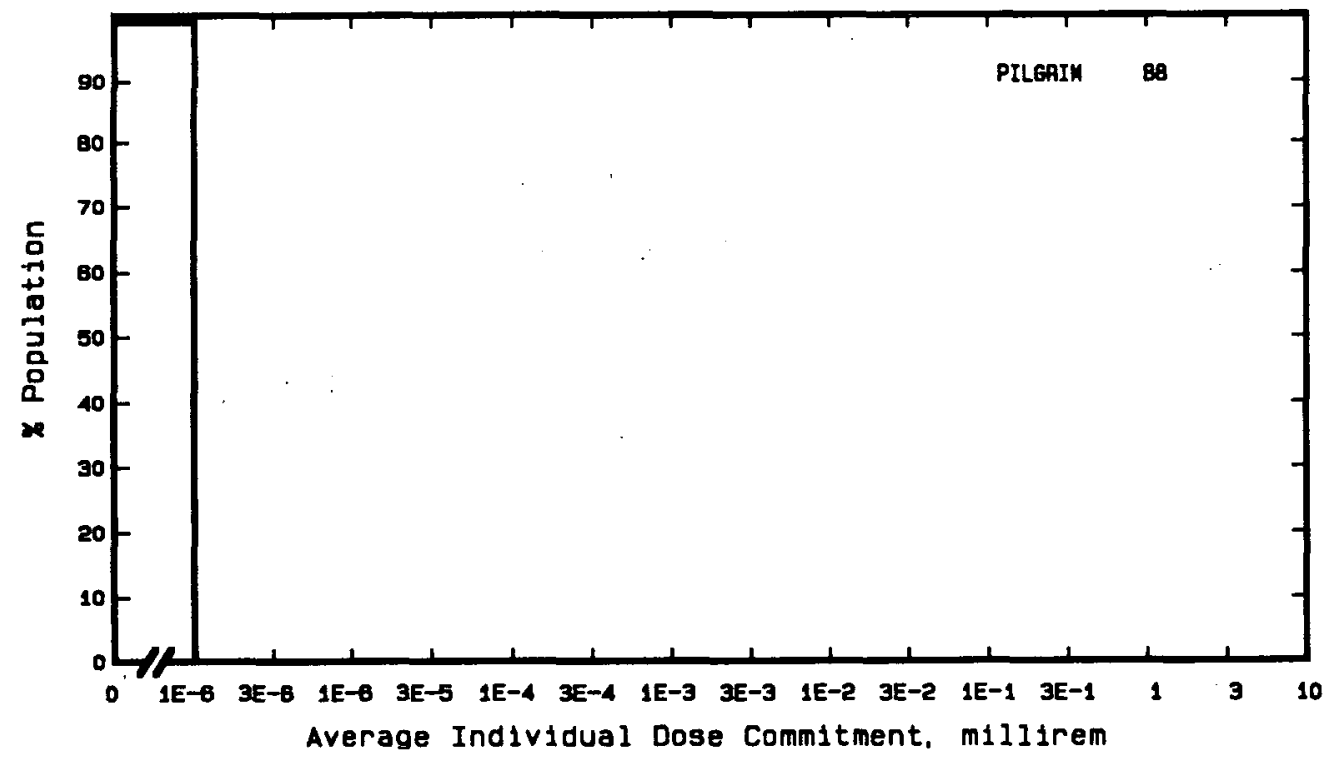


Site: POINT BEACH

MANITOWOC COUNTY, WISCONSIN

Location: $\quad$ N $44.2808^{\circ} \quad$ W $87.5361^{\circ}$

POPULATION DATA

Total Population Within 2-to-80-km Region: 6.3E5

Major Metropolitan Centers Within Region:

\begin{tabular}{lrrl}
\multicolumn{1}{c}{ Center } & Population & \multicolumn{2}{c}{ Location } \\
\cline { 2 - 5 } & & & \\
Greenbay SMSA & 180,000 & $47 \mathrm{~km}$ & NW \\
Appleton & 61,000 & $72 \mathrm{~km}$ & W \\
Sheboygan & 49,000 & $60 \mathrm{~km}$ & SSW \\
Manitowoc & 34,000 & $24 \mathrm{~km}$ & SSW \\
Neenah & 22,000 & $75 \mathrm{~km}$ & W
\end{tabular}

SITE-SPECIFIC DATA - AIRBORNE PATHWAYS

Average Annual State Production

of Crops and Animal Products

In $80-\mathrm{km}$ Radius Circle

Regional Productivity Factor:

Animal Grazing Factor:
Veg: $7.2 E 7$ kilogram

Milk: $1.2 \mathrm{E} 9$ liter

Meat: $1.0 \mathrm{E} 8 \mathrm{ki}$ logram

0.5

0.5

Meteorology Period of Record: 19 APR $67-18$ APR 69 Recovery: 83\%

Drinking Water:

Fish:
Average Dilution Flow from Plants: $770 \mathrm{ft}^{3} / \mathrm{s}$

Exposed Population: 270,000

Dilution Factor: $2.6 \mathrm{E}-3(\mathrm{a})$

Edible Harvest: $6.7 \mathrm{E} 4$ (b) $\mathrm{kg} / \mathrm{yr}$

Dilution Factor: $0.013(\mathrm{c})$

(a) Drinking water dilution factor est imated by averaging dilution factor derived from FES (1972), suitably weighted for population.

(b) Includes both sport and commercial fish catch from FES.

(c) Dilution factor estimated by averaging sport and commercial dilution factor derived from FES. 
POPULATION DOSE-COMMITMENT ESTIMATES AND

AVERAGE INDIVIDUAL DOSE-COMMITMENT HISTOGRAM FOR

POINT BEACH 1 AND 2

Dose Commitments (person-rem) from Waterborne Pathways

Total Body GI-LII $\underline{\text { Thyroid }}$ Bone Liver

$\begin{array}{llllll}\text { Infant } & 2.8 \mathrm{E}-04 & 2.7 \mathrm{E}-04 & 3.6 \mathrm{E}-04 & 4.5 \mathrm{E}-05 & 2.9 \mathrm{E}-04 \\ \text { Child } & 3.2 \mathrm{E}-03 & 3.1 \mathrm{E}-03 & 3.7 \mathrm{E}-03 & 9.9 \mathrm{E}-04 & 3.6 \mathrm{E}-03 \\ \text { Teen } & 1.3 \mathrm{E}-03 & 1.2 \mathrm{E}-03 & 1.3 \mathrm{E}-03 & 4.4 \mathrm{E}-04 & 1.6 \mathrm{E}-03 \\ \text { Adult } & 1.2 \mathrm{E}-02 & 1.1 \mathrm{E}-02 & 1.1 \mathrm{E}-02 & 2.7 \mathrm{E}-03 & 1.2 \mathrm{E}-02 \\ \text { TOTAL } & 1.7 \mathrm{E}-02 & 1.5 \mathrm{E}-02 & 1.7 \mathrm{E}-02 & 4.2 \mathrm{E}-03 & 1.8 \mathrm{E}-02\end{array}$

Dose Commitments (person-rem) from Airborne Pathways

Total Body GI-LII Thyroid Bone Liver Lung

$\begin{array}{lllllll}\text { Infant } & 3.8 \mathrm{E}-04 & 3.8 \mathrm{E}-04 & 5.5 \mathrm{E}-04 & 4.8 \mathrm{E}-05 & 4.1 \mathrm{E}-04 & 3.9 \mathrm{E}-04 \\ \text { Child } & 6.0 \mathrm{E}-03 & 5.9 \mathrm{E}-03 & 7.1 \mathrm{E}-03 & 5.3 \mathrm{E}-04 & 6.2 \mathrm{E}-03 & 6.0 \mathrm{E}-03 \\ \text { Teen } & 3.6 \mathrm{E}-03 & 3.6 \mathrm{E}-03 & 4.1 \mathrm{E}-03 & 2.6 \mathrm{E}-04 & 3.7 \mathrm{E}-03 & 3.7 \mathrm{E}-03 \\ \text { Adult } & 2.0 \mathrm{E}-02 & 2.0 \mathrm{E}-02 & 2.1 \mathrm{E}-02 & 1.3 \mathrm{E}-03 & 2.0 \mathrm{E}-02 & 2.0 \mathrm{E}-02 \\ \text { TOTAL } & 3.0 \mathrm{E}-02 & 3.0 \mathrm{E}-02 & 3.3 \mathrm{E}-02 & 2.1 \mathrm{E}-03 & 3.0 \mathrm{E}-02 & 3.0 \mathrm{E}-02\end{array}$

Production/Consumption factors:

Produce: 0.29 Milk: $7.0 \quad$ Meat: 1.0

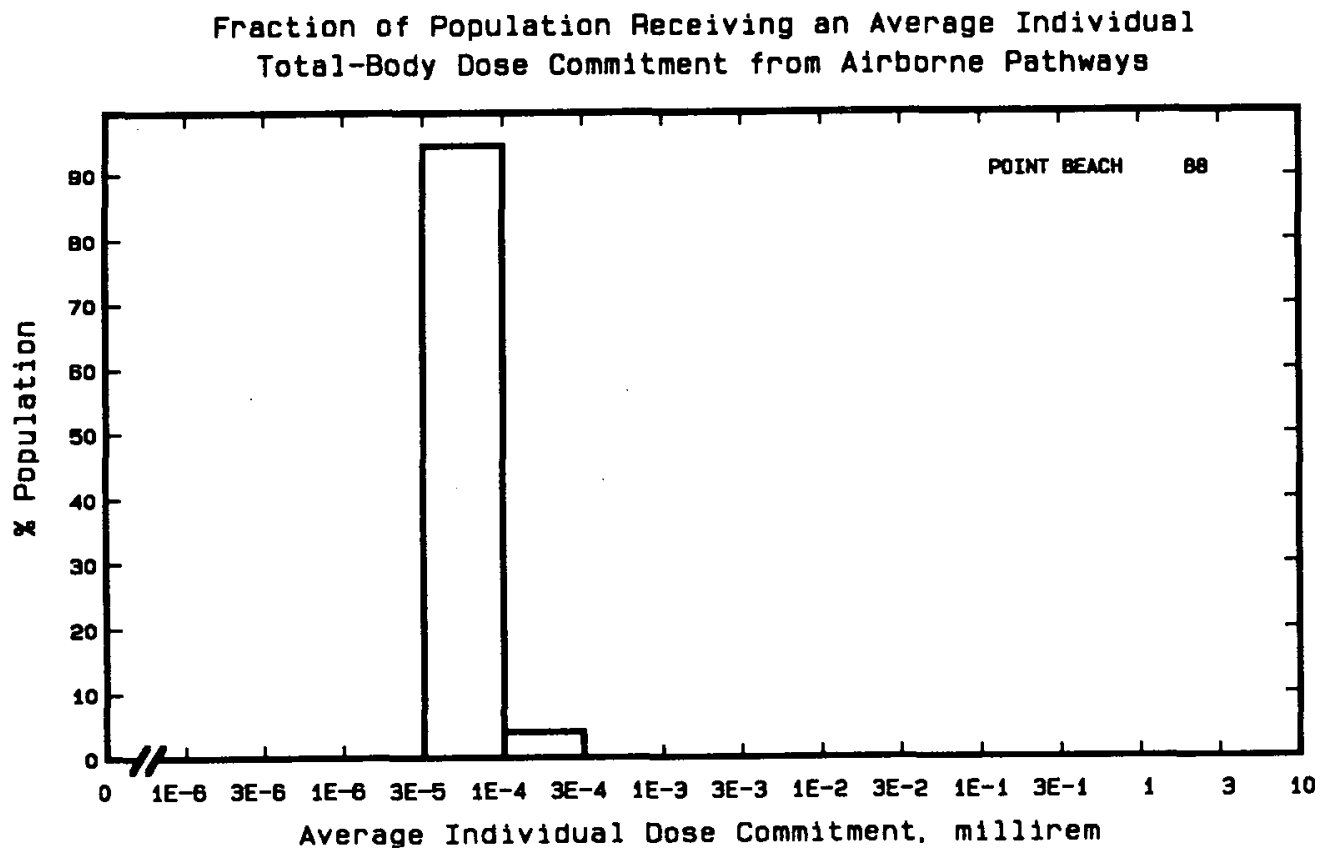


Site: PRAIRIE ISLAND

RED WING, MINNESOTA

Location: N $44.6219^{\circ} \quad$ W $92.6331^{\circ}$

POPULATION DATA

Total Population Within 2-to-80-km Region: 2.3E6

Major Metropolitan Centers Within Region:

Center

Population

Location

Minneapolis-St. Paul SMSA $2,200,000$

Rochester SMSA

98,000

Owatonna

19,000

Faribault

16,000

Redwing

14,000

$63 \mathrm{~km} \mathrm{NW}$

$68 \mathrm{~km} \quad$ SSE

$77 \mathrm{~km} \quad \mathrm{SW}$

$63 \mathrm{~km} \quad \mathrm{SW}$

$10 \mathrm{~km} \quad \mathrm{SE}$

\section{SITE-SPECIFIC DATA - AIRBORNE PATHWAYS}

Average Annual State Production

of Crops and Animal Products

In 80-km Radius Circle

Veg: $1.2 \mathrm{E} 8$ kilogram

Milk: 4.0E8 liter

Meat: 1.0E8 kilogram

Regional Productivity Factor:

Animal Grazing Factor:

1

0.5

Meteorology Period of Record: 22 MAR 74 - 21 MAR 75 Recovery: $65 \%$

\section{SITE-SPECIFIC DATA - WATERBORNE PATHWAYS via MISSISSIPPI RIVER}

Average River Flow at Site: $15,000 \mathrm{ft}^{3} / \mathrm{s}$

Drinking Water:

None

Fish:

Edible Harvest: $6.8 \mathrm{E} 5 \mathrm{~kg} / \mathrm{yr}$

Dilution Factor: 1 


\section{POPULATION DOSE-COMMITMENT ESTIMATES AND AVERAGE INDIVIDUAL DOSE-COMMITMENT HISTOGRAM FOR PRAIRIE ISLAND 1 AND 2}

Dose Commitments (person-rem) from Waterborne Pathways

Total Body GI-LII $\underline{\text { Thyroid }}$ Bone Liver

$\begin{array}{llllll}\text { Infant } & 0.0 \mathrm{E}+00 & 0.0 \mathrm{E}+00 & 0.0 \mathrm{E}+00 & 0.0 \mathrm{E}+00 & 0.0 \mathrm{E}+00 \\ \text { Child } & 4.2 \mathrm{E}-04 & 7.6 \mathrm{E}-04 & 2.3 \mathrm{E}-04 & 1.2 \mathrm{E}-03 & 6.9 \mathrm{E}-04 \\ \text { Teen } & 3.3 \mathrm{E}-04 & 1.3 \mathrm{E}-03 & 2.0 \mathrm{E}-04 & 6.9 \mathrm{E}-04 & 5.6 \mathrm{E}-04 \\ \text { Adult } & 2.4 \mathrm{E}-03 & 1.1 \mathrm{E}-02 & 1.6 \mathrm{E}-03 & 4.1 \mathrm{E}-03 & 3.7 \mathrm{E}-03 \\ \text { TOTAL } & 3.1 \mathrm{E}-03 & 1.3 \mathrm{E}-02 & 2.1 \mathrm{E}-03 & 6.0 \mathrm{E}-03 & 4.9 \mathrm{E}-03\end{array}$

Dose Commitments (person-rem) from Airborne Pathways Total Body GI-LII Thyroid Bone Liver

$\begin{array}{lllllll}\text { Infant } & 9.6 \mathrm{E}-04 & 9.6 \mathrm{E}-04 & 9.6 \mathrm{E}-04 & 4.5 \mathrm{E}-06 & 9.6 \mathrm{E}-04 & 9.6 \mathrm{E}-04 \\ \text { Child } & 1.5 \mathrm{E}-02 & 1.5 \mathrm{E}-02 & 1.5 \mathrm{E}-02 & 1.8 \mathrm{E}-04 & 1.5 \mathrm{E}-02 & 1.5 \mathrm{E}-02 \\ \text { Teen } & 9.4 \mathrm{E}-03 & 9.4 \mathrm{E}-03 & 9.4 \mathrm{E}-03 & 6.8 \mathrm{E}-05 & 9.4 \mathrm{E}-03 & 9.4 \mathrm{E}-03 \\ \text { Adult } & 5.1 \mathrm{E}-02 & 5.1 \mathrm{E}-02 & 5.1 \mathrm{E}-02 & 2.7 \mathrm{E}-04 & 5.1 \mathrm{E}-02 & 5.1 \mathrm{E}-02 \\ \text { TOTAL } & 7.7 \mathrm{E}-02 & 7.6 \mathrm{E}-02 & 7.7 \mathrm{E}-02 & 5.2 \mathrm{E}-04 & 7.6 \mathrm{E}-02 & 7.6 \mathrm{E}-02\end{array}$

Production/Consumption factors:
Produce: 0.27
Milk: 1.3
Meat: 0.57

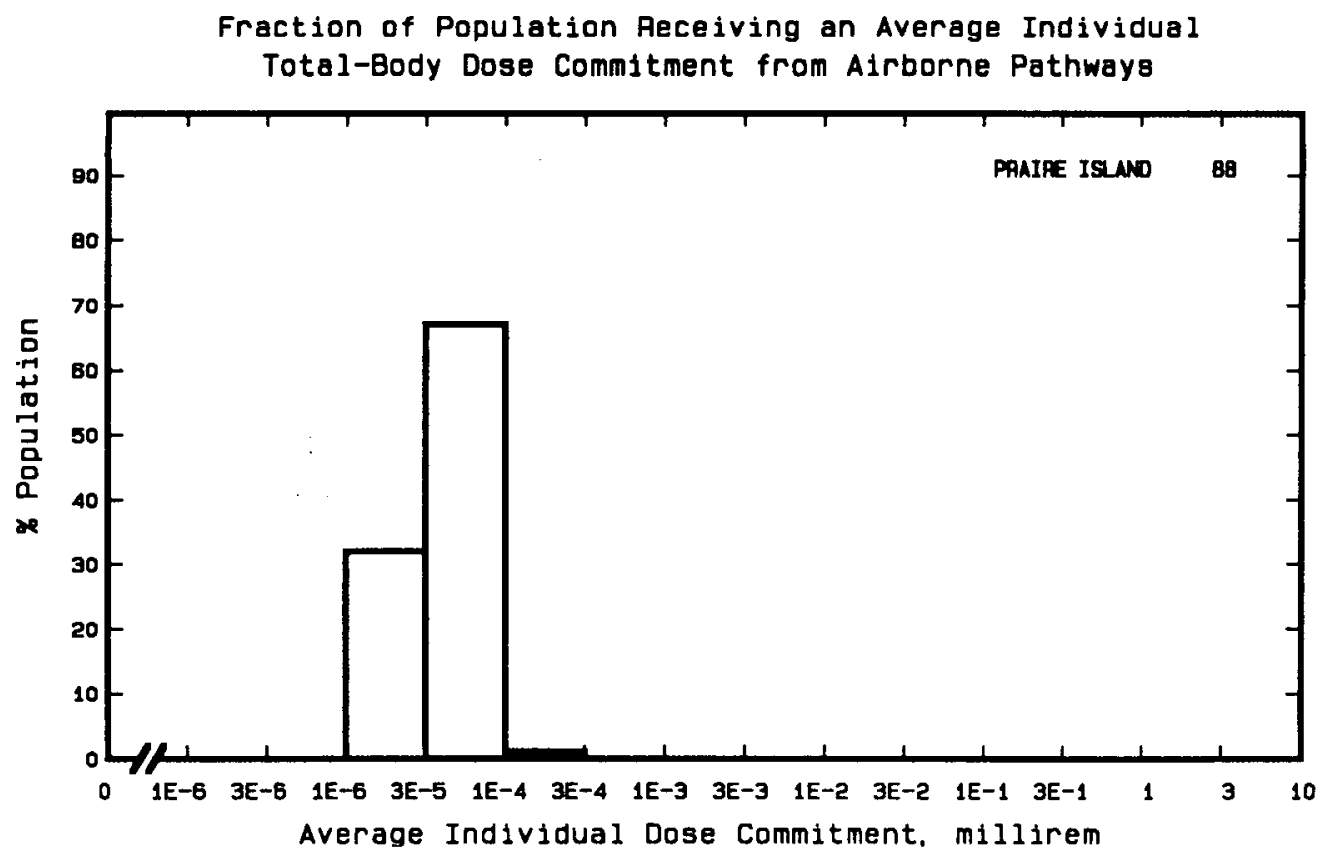


Site: QUAD CITIES

ROCK ISLAND, ILLINOIS

Location: $\quad N 41.7261^{\circ} \quad$ W $90.3100^{\circ}$

POPULATION DATA

Total Population Within 2-to-80-km Region: 7.3E5

Major Metropolitan Centers Within Region:

Center

Population

Location

Davenport-Rock Is land-Mol ine SMSA 390,000

Muscatine

23,000

16,000

Sterling

16,000

Kewanee

15,000

$30 \mathrm{~km} \mathrm{SW}$

$70 \mathrm{~km} \quad$ WSW

$52 \mathrm{~km} \quad \mathrm{E}$

$70 \mathrm{~km} \quad \mathrm{E}$

$62 \mathrm{~km} \quad$ SSE

SITE-SPECIFIC DATA - AIRBORNE PATHWAYS

Average Annual State Production

of Crops and Animal Products

In $80-\mathrm{km}$ Radius Circle

Veg: $1.1 \mathrm{E} 8 \mathrm{ki}$ logram

Milk: $1.8 \mathrm{E} 8$ liter

Meat: $1.9 \mathrm{E} 8 \mathrm{kilogram}$

Regional Productivity Factor:

Animal Grazing Factor:

1

0.5

Meteorology Period of Record: 1 JAN 74 - 31 DEC 75 Recovery: $88 \%$

SITE-SPECIFIC DATA - WATERBORNE PATHWAYS via MISSISSIPPI RIVER

Average River Flow

at Site: $47,000 \mathrm{ft}^{3} / \mathrm{s}$

Drinking Water:

Exposed Population: 390,000 (a)

Dilution Factor: 1

Fish:

Edible Harvest: $2.1 \mathrm{Eg}$ (b) $\mathrm{kg} / \mathrm{yr}$

Dilution Factor: 0.5 (b)

(a) All people in Davenport SMSA assumed to drink river water.

(b) Assumes $1 / 2$ fish harvest caught below plant. 
POPULATION DOSE-COMMITMENT ESTIMATES AND

AVERAGE INDIVIDUAL DOSE-COMMITMENT HISTOGRAM FOR

QUAD CITIES 1 AND 2

Dose Commitments (person-rem) from Waterborne Pathways

Total Body GI-LII $\underline{\text { Thyroid }}$ Bone Liver

$\begin{array}{llllll}\text { Infant } & 9.5 \mathrm{E}-05 & 6.6 \mathrm{E}-05 & 5.7 \mathrm{E}-05 & 2.4 \mathrm{E}-04 & 1.9 \mathrm{E}-04 \\ \text { Child } & 2.7 \mathrm{E}-03 & 9.7 \mathrm{E}-04 & 6.2 \mathrm{E}-04 & 1.3 \mathrm{E}-02 & 1.1 \mathrm{E}-02 \\ \text { Teen } & 3.2 \mathrm{E}-03 & 7.1 \mathrm{E}-04 & 2.3 \mathrm{E}-04 & 6.6 \mathrm{E}-03 & 8.2 \mathrm{E}-03 \\ \text { Adult } & 3.4 \mathrm{E}-02 & 6.2 \mathrm{E}-03 & 2.0 \mathrm{E}-03 & 3.9 \mathrm{E}-02 & 4.9 \mathrm{E}-02 \\ \text { TOTAL } & 4.0 \mathrm{E}-02 & 8.02 \mathrm{E}-03 & 2.9 \mathrm{E}-03 & 5.9 \mathrm{E}-02 & 6.8 \mathrm{E}-02\end{array}$

Dose Commitments (person-rem) from Airborne Pathways

Total Body GI-LII Thyroid Bone Liver Lung

$\begin{array}{lllllll}\text { Infant } & 1.7 \mathrm{E}-04 & 1.7 \mathrm{E}-04 & 2.5 \mathrm{E}-03 & 1.0 \mathrm{E}-04 & 2.2 \mathrm{E}-04 & 2.1 \mathrm{E}-04 \\ \text { Child } & 3.2 \mathrm{E}-03 & 3.3 \mathrm{E}-03 & 2.5 \mathrm{E}-02 & 1.6 \mathrm{E}-03 & 3.6 \mathrm{E}-03 & 3.8 \mathrm{E}-03 \\ \text { Teen } & 2.0 \mathrm{E}-03 & 2.2 \mathrm{E}-03 & 1.1 \mathrm{E}-02 & 7.7 \mathrm{E}-04 & 2.1 \mathrm{E}-03 & 2.5 \mathrm{E}-03 \\ \text { Adult } & 1.1 \mathrm{E}-02 & 1.2 \mathrm{E}-02 & 4.3 \mathrm{E}-02 & 3.9 \mathrm{E}-03 & 1.1 \mathrm{E}-02 & 1.3 \mathrm{E}-02 \\ \text { TOTAL } & 1.6 \mathrm{E}-02 & 1.8 \mathrm{E}-02 & 8.1 \mathrm{E}-02 & 6.3 \mathrm{E}-03 & 1.7 \mathrm{E}-02 & 2.0 \mathrm{E}-02\end{array}$

Production/Consumption factors:

Produce: $\quad 0.80$

Milk: 1.8

Meat: 3.2

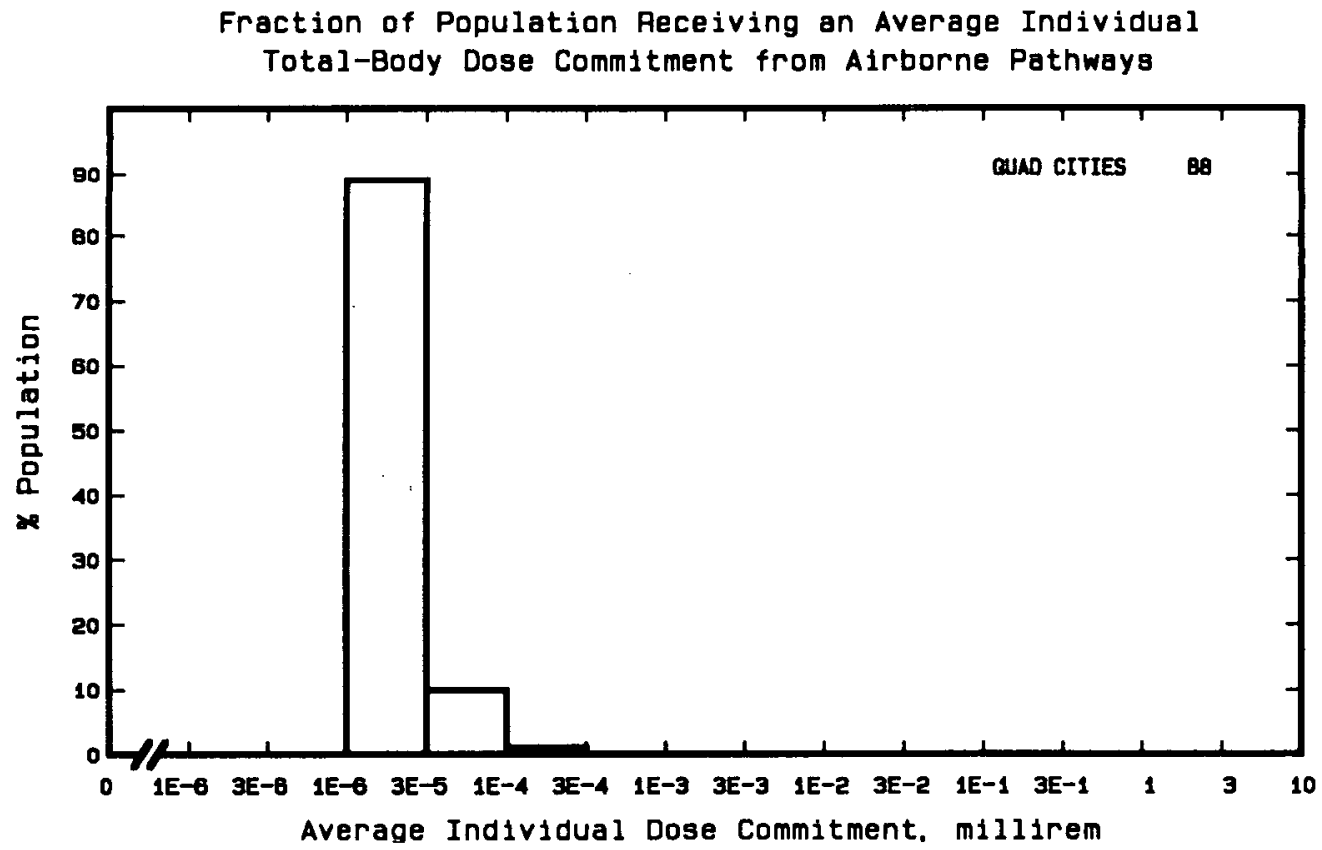


Site: RANCHO SECO

SACRAMENTO COUNTY, CALIFORNIA

Location: N $38.3444^{\circ} \quad$ W $121.1200^{\circ}$

POPULATION DATA

Total Population Within 2-to-80-km Region: 2.1E6

Major Metropolitan Centers Within Region:

\begin{tabular}{lrrl}
\multicolumn{1}{c}{ Center } & Population & \multicolumn{2}{c}{ Location } \\
\cline { 2 - 4 } & & & \\
Sacramento SMSA & $1,300,000$ & $42 \mathrm{~km}$ & NW \\
Stockton SMSA & 440,000 & $45 \mathrm{~km}$ & SSW \\
Modesto SMSA & 330,000 & $79 \mathrm{~km}$ & S \\
Antioch & 50,000 & $71 \mathrm{~km}$ & WSW \\
Davis & 43,000 & $58 \mathrm{~km}$ & WNW
\end{tabular}

SITE-SPECIFIC DATA - AIRBORNE PATHWAYS

Average Annual State Production

of Crops and Animal Products

In $80-\mathrm{km}$ Radius Circle

Veg: $4.8 \mathrm{E} 7 \mathrm{ki}$ logram

Milk: 2.3E8 liter

Meat: $5.0 E 7$ kilogram

Regional Productivity Factor:

Animal Grazing Factor:

1

0.9

Meteorology Period of Record: 1 FEB 75 - 31 JAN 76 Recovery: 98\%

SITE SPECIFIC DATA - WATERBORNE PATHWAYS via COSUMNES and MOKELUMNE

RIVERS

Average Dilution Flow

from Plant: $19 \mathrm{ft}^{3} / \mathrm{s}$

Drinking Water: None

Fish:

Edible Harvest: (a)

Dilution Factor: 0.005

(a) One percent of population is assumed to obtain fish from river. Average individual consumption rate of $7.3 \mathrm{~kg} / \mathrm{yr}$ given in FES (1973) used in lieu of catch data. 
POPULATION DOSE-COMMITMENT ESTIMATES AND

AVERAGE INDIVIDUAL DOSE-COMMITMENT HISTOGRAM FOR

RANCHO SECO

Dose Commitments (person-rem) from Waterborne Pathways

Total Body GI-LII Ihyroid Bone Liver

$\begin{array}{llllll}\text { Infant } & 0.0 \mathrm{E}+00 & 0.0 \mathrm{E}+00 & 0.0 \mathrm{E}+00 & 0.0 \mathrm{E}+00 & 0.0 \mathrm{E}+00 \\ \text { Child } & 9.0 \mathrm{E}-04 & 8.5 \mathrm{E}-05 & 1.6 \mathrm{E}-04 & 5.2 \mathrm{E}-03 & 5.4 \mathrm{E}-03 \\ \text { Teen } & 1.7 \mathrm{E}-03 & 1.2 \mathrm{E}-04 & 1.2 \mathrm{E}-04 & 3.1 \mathrm{E}-03 & 4.5 \mathrm{E}-03 \\ \text { Adult } & 1.8 \mathrm{E}-02 & 9.4 \mathrm{E}-04 & 8.6 \mathrm{E}-04 & 1.8 \mathrm{E}-02 & 2.7 \mathrm{E}-02 \\ \text { TOTAL } & 2.1 \mathrm{E}-02 & 1.1 \mathrm{E}-03 & 1.1 \mathrm{E}-03 & 2.6 \mathrm{E}-02 & 3.6 \mathrm{E}-02\end{array}$

Dose Commitments (person-rem) from Airborne Pathways

Total Body GI-LII Ihyroid Bone Liver lung

$\begin{array}{lllllll}\text { Infant } & 8.5 \mathrm{E}-04 & 8.5 \mathrm{E}-04 & 1.1 \mathrm{E}-03 & 7.2 \mathrm{E}-04 & 8.5 \mathrm{E}-04 & 9.1 \mathrm{E}-04 \\ \text { Child } & 9.7 \mathrm{E}-03 & 9.7 \mathrm{E}-03 & 1.1 \mathrm{E}-02 & 8.0 \mathrm{E}-03 & 9.7 \mathrm{E}-03 & 1.1 \mathrm{E}-02 \\ \text { Teen } & 7.0 \mathrm{E}-03 & 7.0 \mathrm{E}-03 & 7.5 \mathrm{E}-03 & 5.9 \mathrm{E}-03 & 7.0 \mathrm{E}-03 & 8.1 \mathrm{E}-03 \\ \text { Adult } & 4.1 \mathrm{E}-02 & 4.1 \mathrm{E}-02 & 4.3 \mathrm{E}-02 & 3.5 \mathrm{E}-02 & 4.1 \mathrm{E}-02 & 4.5 \mathrm{E}-02 \\ \text { TOTAL } & 5.9 \mathrm{E}-02 & 5.9 \mathrm{E}-02 & 6.3 \mathrm{E}-02 & 5.0 \mathrm{E}-02 & 5.9 \mathrm{E}-02 & 6.5 \mathrm{E}-02\end{array}$

Production/Consumption factors:

Produce: $0.12 \quad$ Milk: $0.84 \quad$ Meat: 0.30

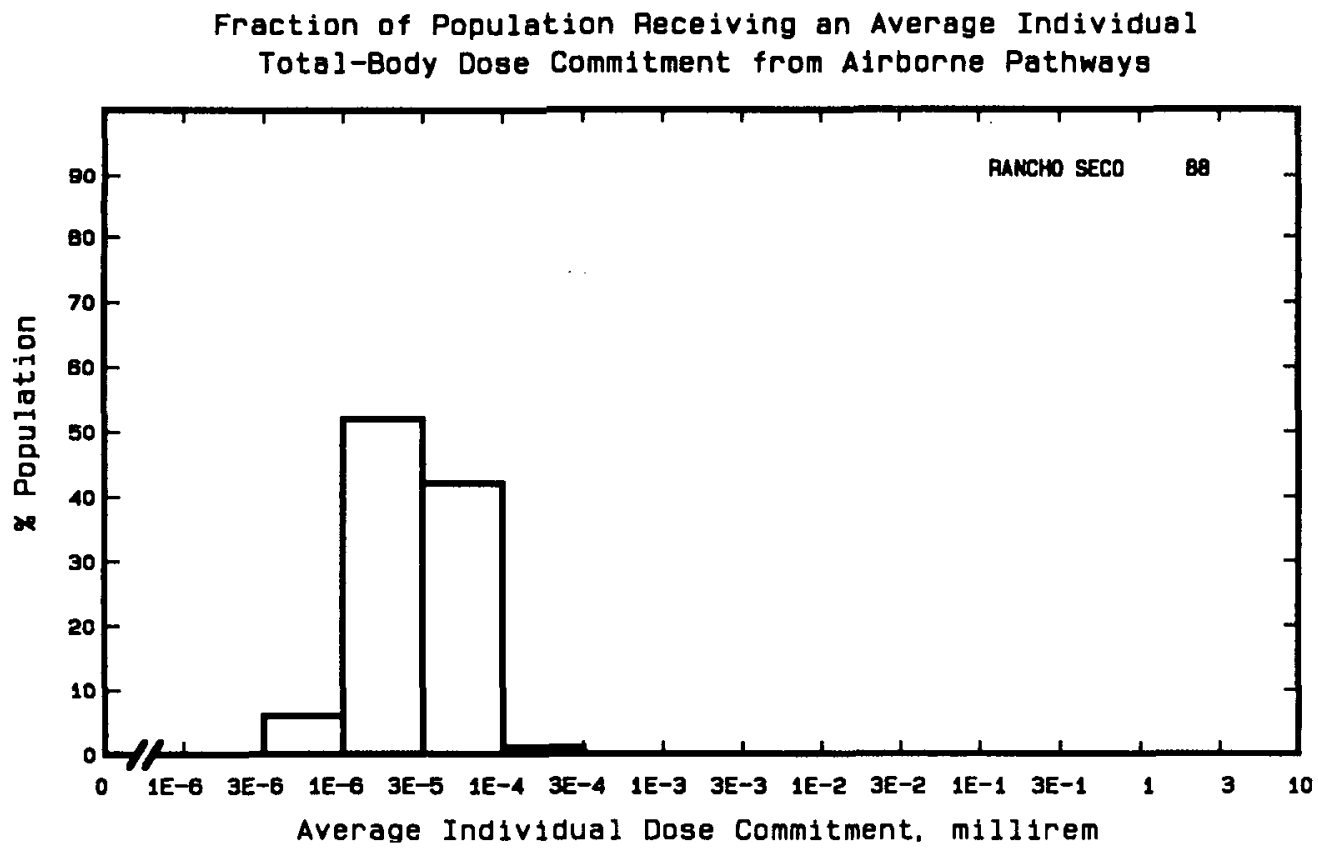


Site: RIVER BEND

ST. FRANCISVILLE, LOUISIANA

Location: N $30.7572^{\circ} \quad$ W $91.3317^{\circ}$

POPULATION DATA

Total Population Within 2-to-80-km Region: 7.6E5

Major Metropolitan Centers Within Region:

Center

Population

Location

Baton Rouge

Denham Springs

240,000

9,000

$38 \mathrm{~km} \quad$ SSE

$39 \mathrm{~km} \quad \mathrm{SE}$

SITE-SPECIFIC DATA - AIRBORNE PATHWAYS

Average Annual State Production

Veg: $1.8 \mathrm{E} 6$ kilogram

of Crops and Animal Products

In $80-\mathrm{km}$ Radius Circle

Milk: $7.8 E 7$ liter

Meat: $6.1 \mathrm{E} 7$ kilogram

Regional Productivity Factor:

0.9

Animal Grazing Factor:

0.75

Meteorology Period of Record: 17 MAR 77 - 16 MAR 79 Recovery: 99\%

SITE-SPECIFIC DATA - WATERBORNE PATHWAYS via MISSISSIPPI RIVER

Average River Flow

at Site: $420,000 \mathrm{ft}^{3} / \mathrm{s}$

Drinking Water: None

Fish:

Edible Harvest: $9.1^{(\mathrm{a})} \mathrm{kg} / \mathrm{yr}$

Dilution Factor: 1.0

(a) Individual consumption rate as given in FES (Includes shellfish). 


\section{POPULATION DOSE-COMMITMENT ESTIMATES AND \\ AVERAGE INDIVIDUAL DOSE-COMMITMENT HISTOGRAM FOR \\ RIVER BEND}

Dose Commitments (person-rem) from Waterborne Pathways

Total Body GI-LII Thyroid Bone Liver

$\begin{array}{llllll}\text { Infant } & 0.0 \mathrm{E}+00 & 0.0 \mathrm{E}+00 & 0.0 \mathrm{E}+00 & 0.0 \mathrm{E}+00 & 0.0 \mathrm{E}+00 \\ \text { Child } & 1.2 \mathrm{E}-04 & 1.4 \mathrm{E}-04 & 1.5 \mathrm{E}-06 & 6.2 \mathrm{E}-05 & 2.0 \mathrm{E}-04 \\ \text { Teen } & 8.3 \mathrm{E}-05 & 3.2 \mathrm{E}-04 & 1.3 \mathrm{E}-06 & 4.2 \mathrm{E}-05 & 1.9 \mathrm{E}-04 \\ \text { Adult } & 5.0 \mathrm{E}-04 & 2.9 \mathrm{E}-03 & 1.0 \mathrm{E}-05 & 2.8 \mathrm{E}-04 & 1.2 \mathrm{E}-03 \\ \text { TOTAL } & 7.0 \mathrm{E}-04 & 3.3 \mathrm{E}-03 & 1.3 \mathrm{E}-05 & 3.8 \mathrm{E}-04 & 1.5 \mathrm{E}-03\end{array}$

Dose Commitments (person-rem) from Airborne Pathways

Total Body GI-LII Thyroid Bone Liver Lung

$\begin{array}{lllllll}\text { Infant } & 1.4 \mathrm{E}-05 & 1.4 \mathrm{E}-05 & 2.7 \mathrm{E}-04 & 4.6 \mathrm{E}-06 & 1.4 \mathrm{E}-05 & 1.5 \mathrm{E}-05 \\ \text { Child } & 1.8 \mathrm{E}-04 & 1.8 \mathrm{E}-04 & 1.7 \mathrm{E}-03 & 4.8 \mathrm{E}-05 & 1.8 \mathrm{E}-04 & 1.9 \mathrm{E}-04 \\ \text { Teen } & 1.2 \mathrm{E}-04 & 1.2 \mathrm{E}-04 & 8.0 \mathrm{E}-04 & 3.2 \mathrm{E}-05 & 1.2 \mathrm{E}-04 & 1.4 \mathrm{E}-04 \\ \text { Adult } & 7.1 \mathrm{E}-04 & 7.2 \mathrm{E}-04 & 3.2 \mathrm{E}-03 & 1.9 \mathrm{E}-04 & 7.2 \mathrm{E}-04 & 7.8 \mathrm{E}-04 \\ \text { 708AL } & 1.0 \mathrm{E}-03 & 1.0 \mathrm{E}-03 & 6.0 \mathrm{E}-03 & 2.7 \mathrm{E}-04 & 1.0 \mathrm{E}-03 & 1.1 \mathrm{E}-03\end{array}$

Production/Consumption factors:

Produce: 0.011 Milk: 0.71 Meat: 0.91

Fraction of Population Receiving an Average Individual Total-Body Dose Commitment from Airborne Pathways

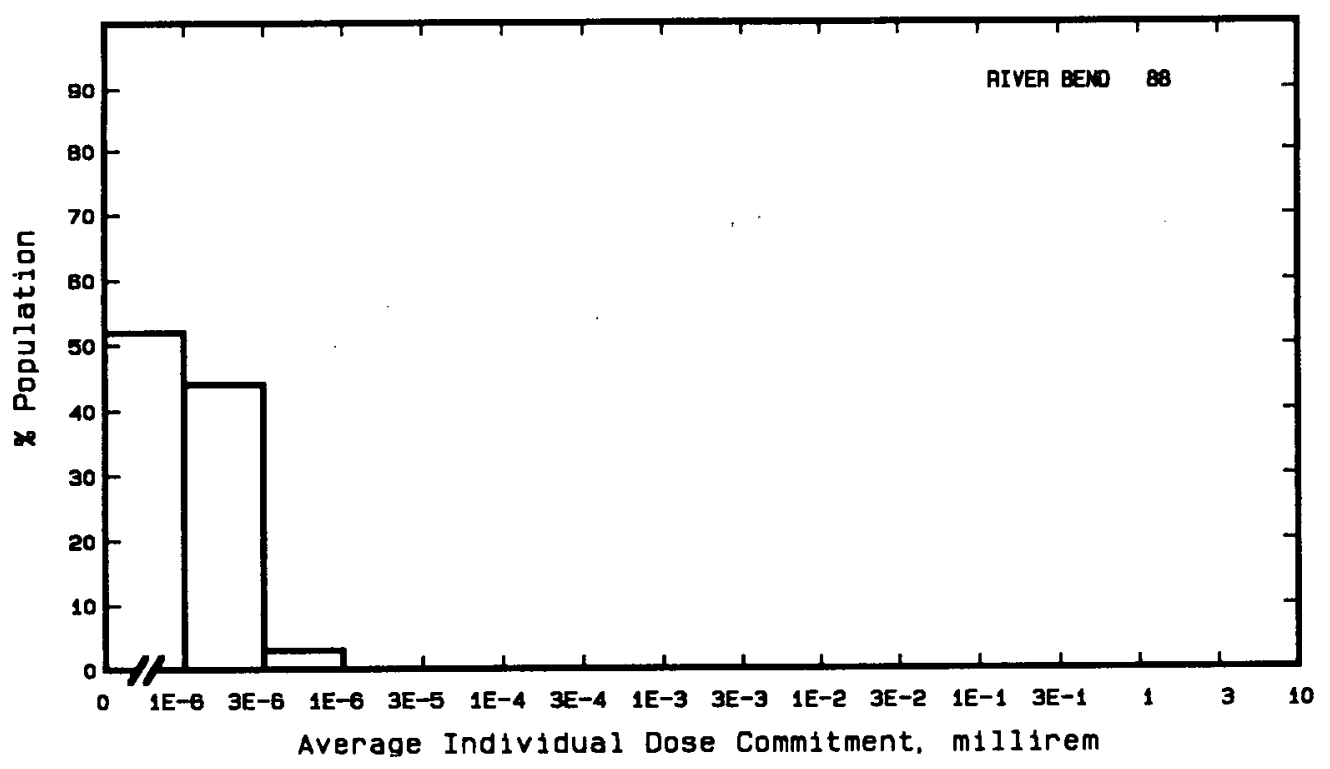


Site: H. B. ROBINSON

HARTSVILLE, SOUTH CAROLINA

Location: $\quad$ N $34.4858^{\circ} \quad$ W $80.1586^{\circ}$

POPULATION DATA

Total Population Within 2-to-80-km Region: 7.3E5

Major Metropolitan Centers Within Region:

Center

Florence

Sumter

Monroe

Lancaster
Population

32,000

27,000

14,000

10,000

\section{Location}

$42 \mathrm{~km} \quad$ ESE

$56 \mathrm{~km} \quad \mathrm{SSW}$

$74 \mathrm{~km} \quad \mathrm{NNW}$

$66 \mathrm{~km} \cdot \mathrm{WNW}$

SITE-SPECIFIC DATA - AIRBORNE PATHWAYS

Average Annual State Production

of Crops and Animal Products

In $80-\mathrm{km}$ Radius Circle

Regional Productivity Factor:

Animal Grazing Factor:
Veg: $7.4 \mathrm{E} 6$ kilogram

Milk: 5.7E7 liter

Meat: 5.0E7 kilogram

1

0.8

Meteorology Period of Record: 1 JAN 75 - 31 DEC 75 Recovery: $94 \%$

SITE-SPECIFIC DATA - WATERBORNE PATHWAYS via LAKE ROBINSON

Average Dilution Flow

from Plant: $940 \mathrm{ft}^{3} / \mathrm{s}$

Drinking Water:

None

Fish:

Edible Harvest: $1.8(\mathrm{a}) \mathrm{kg} / \mathrm{yr}$

Dilution Factor: 0.02 (b)

(a) Average individual consumption rate as given in the FES (1975) used in lieu of catch data.

(b) Ten percent of population obtain fish taken from water diluted by a factor of 0.2 according to FES. 


\section{POPULATION DOSE-COMMITMENT ESTIMATES AND AVERAGE INDIVIDUAL DOSE-COMMITMENT HISTOGRAM FOR}

H. B. ROBINSON 2

Dose Commitments (person-rem) from Waterborne Pathways

Total Body GI-LII $\underline{\text { Thyroid }}$ Bone Liver

$\begin{array}{llllll}\text { Infant } & 0.0 \mathrm{E}+00 & 0.0 \mathrm{E}+00 & 0.0 \mathrm{E}+00 & 0.0 \mathrm{E}+00 & 0.0 \mathrm{E}+00 \\ \text { Child } & 2.2 \mathrm{E}-03 & 1.7 \mathrm{E}-03 & 2.2 \mathrm{E}-04 & 9.2 \mathrm{E}-03 & 9.9 \mathrm{E}-03 \\ \text { Teen } & 3.4 \mathrm{E}-03 & 3.4 \mathrm{E}-03 & 1.9 \mathrm{E}-04 & 5.5 \mathrm{E}-03 & 8.3 \mathrm{E}-03 \\ \text { Adult } & 3.5 \mathrm{E}-02 & 2.9 \mathrm{E}-02 & 1.5 \mathrm{E}-03 & 3.2 \mathrm{E}-02 & 5.0 \mathrm{E}-02 \\ \text { TOTAL } & 4.1 \mathrm{E}-02 & 3.4 \mathrm{E}-02 & 1.9 \mathrm{E}-03 & 4.6 \mathrm{E}-02 & 6.8 \mathrm{E}-02\end{array}$

Dose Commitments (person-rem) from Airborne Pathways

Total Body GI-LII Thyroid Bone Liver Lung

$\begin{array}{lllllll}\text { Infant } & 2.8 \mathrm{E}-04 & 2.8 \mathrm{E}-04 & 7.3 \mathrm{E}-04 & 2.6 \mathrm{E}-04 & 2.8 \mathrm{E}-04 & 3.0 \mathrm{E}-04 \\ \text { Child } & 3.2 \mathrm{E}-03 & 3.2 \mathrm{E}-03 & 5.7 \mathrm{E}-03 & 2.9 \mathrm{E}-03 & 3.2 \mathrm{E}-03 & 3.5 \mathrm{E}-03 \\ \text { Teen } & 2.3 \mathrm{E}-03 & 2.3 \mathrm{E}-03 & 3.4 \mathrm{E}-03 & 2.1 \mathrm{E}-03 & 2.3 \mathrm{E}-03 & 2.7 \mathrm{E}-03 \\ \text { Adult } & 1.4 \mathrm{E}-02 & 1.4 \mathrm{E}-02 & 1.8 \mathrm{E}-02 & 1.3 \mathrm{E}-02 & 1.4 \mathrm{E}-02 & 1.5 \mathrm{E}-02 \\ \text { TOTAL } & 2.0 \mathrm{E}-02 & 2.0 \mathrm{E}-02 & 2.7 \mathrm{E}-02 & 1.8 \mathrm{E}-02 & 2.02 \mathrm{E}-02 & 2.2 \mathrm{E}-02\end{array}$

Production/Consumption factors:

Produce: $0.052 \quad$ Milk: 0.60 Meat: 0.85

Fraction of Population Receiving an Average Individual Total-Body Dose Commitment from Alrborne Pathways

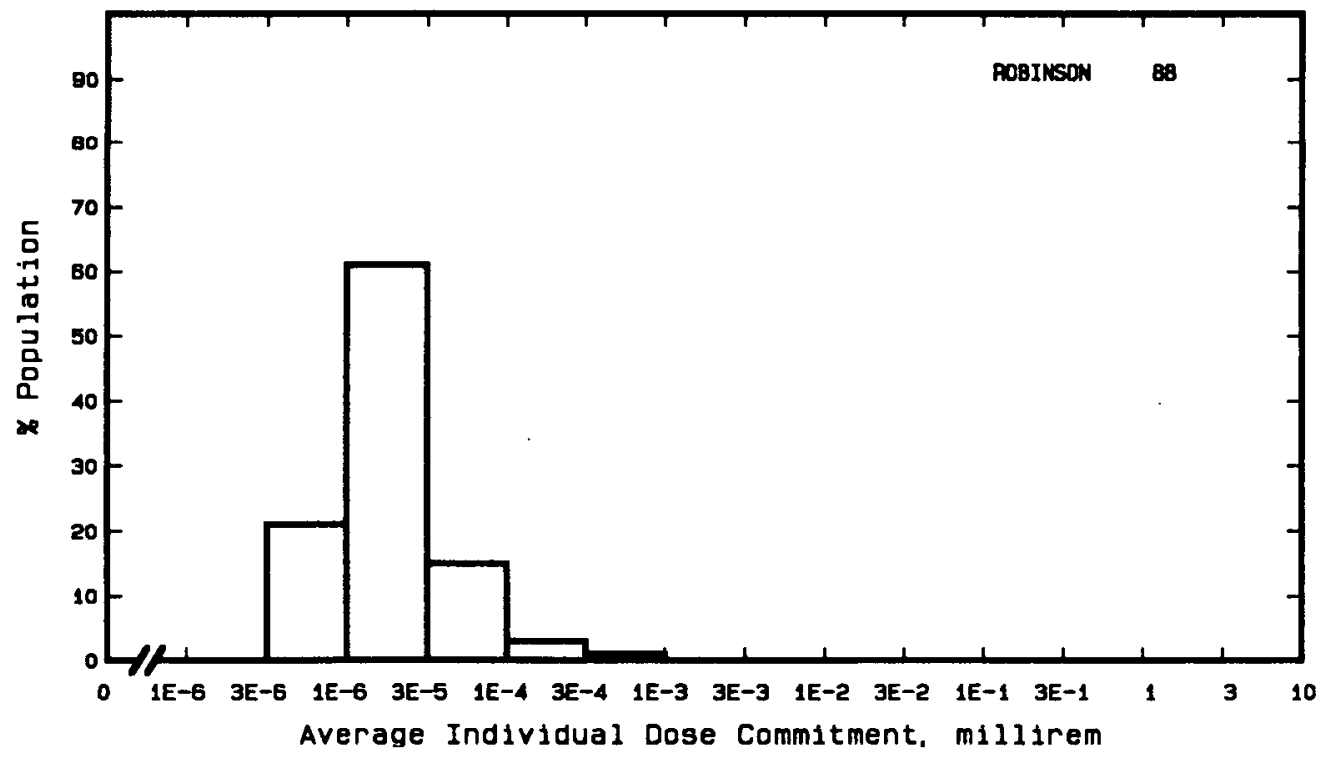


Site: ST. LUCIE

Location: $\quad$ N $27.3486^{\circ}$
FORT PIERCE, FLORIDA

W $80.2464^{\circ}$

POPULATION DATA

Total Population Within 2-to-80-km Region: 7.2E5

Major Metropolitan Centers Within Region:

Center

West Palm Beach

Ft. Pierce

Riviera Beach

Vero Beach

Palm Beach
Population

80,000

43,000

33,000

22,000

13,000
Location

$73 \mathrm{~km} \quad$ SSE

$14 \mathrm{~km} \quad \mathrm{NW}$

$65 \mathrm{~km} \quad \mathrm{SSE}$

$36 \mathrm{~km} \quad \mathrm{NNW}$

$72 \mathrm{~km} \quad$ SSE

\section{SITE-SPECIFIC DATA - AIRBORNE PATHWAYS}

Average Annual State Production

of Crops and Animal Products

In 80-km Radius Circle

Regional Productivity Factor:

Animal Grazing Factor:
Veg: 2.8E7 kilogram

Milk: 1.1 E8 liter

Meat: 7.2E7 kilogram

0.5

1

Meteorology Period of Record: 1 JAN 76 - 31 DEC 76 Recovery: 92\%

Fish:

Invertebrates:

Average Dilution Flow

from Plants: $4,300 \mathrm{ft}^{3} / \mathrm{s}$

Edible Harvest: $2.6 \mathrm{E} 5 \mathrm{~kg} / \mathrm{yr}$

Edible Harvest: $2.7 \mathrm{E} 4 \mathrm{~kg} / \mathrm{yr}$
Dilution Factor: $0.005(\mathrm{a})$

(a) Dilution factors as given in FES (1973). 


\section{POPULATION DOSE-COMMITMENT ESTIMATES AND \\ AVERAGE INDIVIDUAL DOSE-COMMITMENT HISTOGRAM FOR \\ SAINT LUCIE 1 AND 2}

Dose Commitments (person-rem) from Waterborne Pathways

Total Body GI-LII Thyroid Bone Liver

$\begin{array}{llllll}\text { Infant } & 0.0 \mathrm{E}+00 & 0.0 \mathrm{E}+00 & 0.0 \mathrm{E}+00 & 0.0 \mathrm{E}+00 & 0.0 \mathrm{E}+00 \\ \text { Child } & 2.8 \mathrm{E}-05 & 1.7 \mathrm{E}-04 & 1.7 \mathrm{E}-05 & 9.6 \mathrm{E}-05 & 6.5 \mathrm{E}-05 \\ \text { Teen } & 2.2 \mathrm{E}-05 & 3.5 \mathrm{E}-04 & 1.3 \mathrm{E}-05 & 5.5 \mathrm{E}-05 & 5.1 \mathrm{E}-05 \\ \text { Adult } & 1.6 \mathrm{E}-04 & 3.0 \mathrm{E}-03 & 8.6 \mathrm{E}-05 & 3.2 \mathrm{E}-04 & 3.0 \mathrm{E}-04 \\ \text { TOTAL } & 2.1 \mathrm{E}-04 & 3.5 \mathrm{E}-03 & 1.2 \mathrm{E}-04 & 4.7 \mathrm{E}-04 & 4.2 \mathrm{E}-04\end{array}$

Dose Commitments (person-rem) from Airborne Pathways

Total Body GI-LII Thyroid $\underline{\text { Bone }}$ Liver Lung

$\begin{array}{lllllll}\text { Infant } & 4.9 \mathrm{E}-03 & 4.9 \mathrm{E}-03 & 2.1 \mathrm{E}-02 & 4.3 \mathrm{E}-03 & 4.9 \mathrm{E}-03 & 5.1 \mathrm{E}-03 \\ \text { Child } & 5.7 \mathrm{E}-02 & 5.7 \mathrm{E}-02 & 1.5 \mathrm{E}-01 & 4.8 \mathrm{E}-02 & 5.8 \mathrm{E}-02 & 6.1 \mathrm{E}-02 \\ \text { Teen } & 4.2 \mathrm{E}-02 & 4.2 \mathrm{E}-02 & 8.1 \mathrm{E}-02 & 3.5 \mathrm{E}-02 & 4.2 \mathrm{E}-02 & 4.7 \mathrm{E}-02 \\ \text { Adult } & 2.5 \mathrm{E}-01 & 2.5 \mathrm{E}-01 & 3.9 \mathrm{E}-01 & 2.1 \mathrm{E}-01 & 2.5 \mathrm{E}-01 & 2.7 \mathrm{E}-01 \\ \text { TOTAL } & 3.6 \mathrm{E}-01 & 3.6 \mathrm{E}-01 & 6.4 \mathrm{E}-01 & 3.0 \mathrm{E}-01 & 3.6 \mathrm{E}-01 & 3.8 \mathrm{E}-01\end{array}$

Production/Consumption factors:

Produce: $0.10 \quad$ Milk: $0.60 \quad$ Meat: 0.63

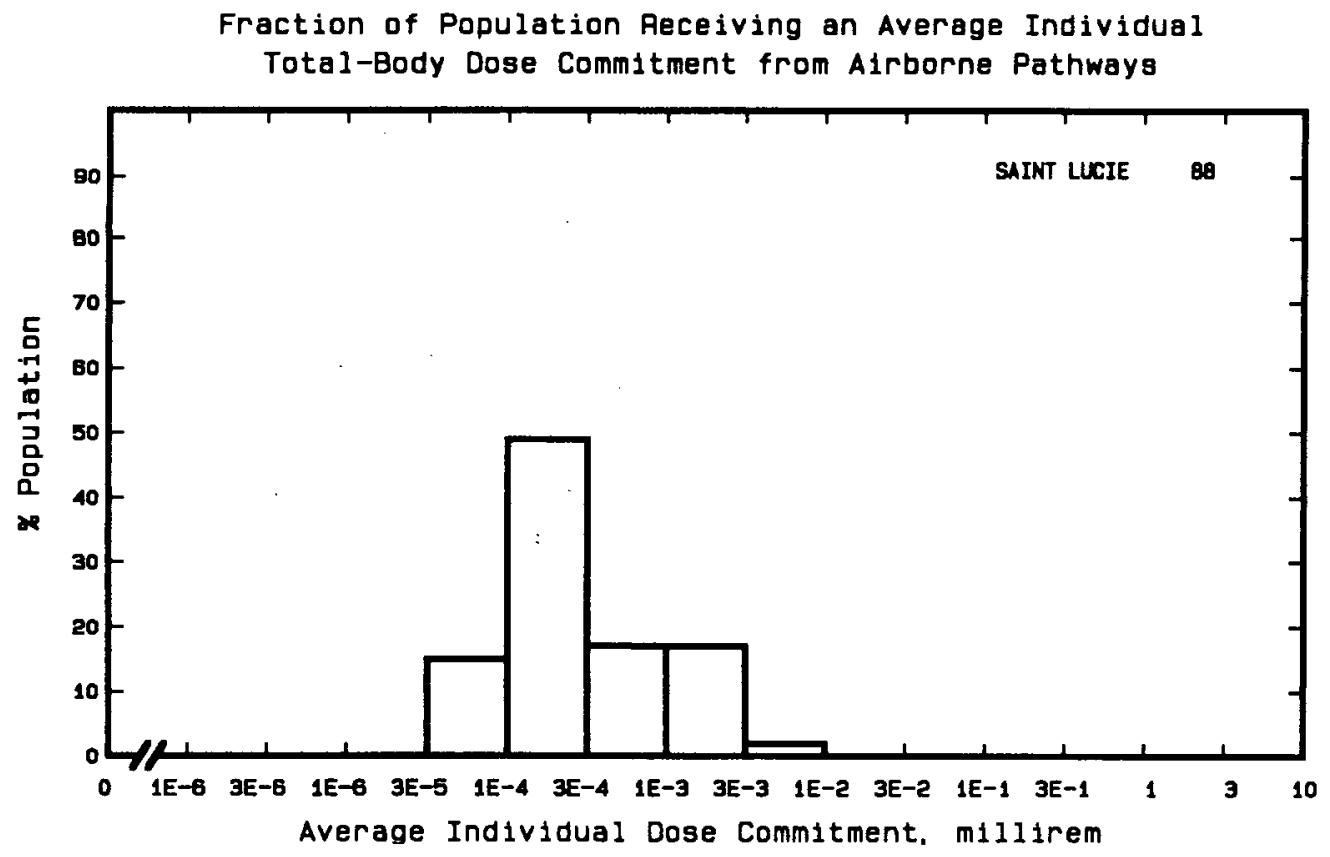


Site: SALEM

SALEM, NEW JERSEY

Location: $\quad$ N $39.4628^{\circ}$ W $75.5358^{\circ}$

POPULATION DATA

Total Population Within 2-to-80-km Region: 4.9E6

Major Metropolitan Centers Within Region:

Center

Philadelphia SMSA

Wilmington SMSA

Vineland-Millville-Bridgeton SMSA

Chester

Norristown
Population

$4,900,000$

420,000

130,000

48,000

36,000
Location

$63 \mathrm{~km} \quad \mathrm{NNE}$

$32 \mathrm{~km} \quad \mathrm{NNW}$

$48 \mathrm{~km} \quad \mathrm{E}$

$45 \mathrm{~km} \quad \mathrm{NNE}$

$74 \mathrm{~km} \quad \mathrm{~N}$

SITE-SPECIFIC DATA - AIRBORNE PATHWAYS

Average Annual State Production

of Crops and Animal Products

In 80-km Radius Circle

Regional Productivity Factor:

Animal Grazing Factor:
Veg: 7.4E7 kilogram

Milk: 2.7E8 liter

Meat: 2.4E7 kilogram

0.9

0.6

Meteorology Period of Record: 1 JUN 70 - 31 MAY 71 Recovery: 95\%

SITE-SPECIFIC DATA - WATERBORNE PATHWAYS via DELAWARE RIVER ESTUARY

Average River Flow

at Site: $16,000 \mathrm{ft}^{3} / \mathrm{s}$

Fish:

Edible Harvest: $3.6 \mathrm{E} 5 \mathrm{~kg} / \mathrm{yr}$

Dilution Factor: 1

Invertebrates: (a)

Edible Harvest: $1.6 \mathrm{E} 5 \mathrm{~kg} / \mathrm{yr}$

Dilution Factor: 1

(a) Environment primarily salt water so invertebrates considered in lieu of drinking water. 


\section{POPULATION DOSE-COMMITMENT ESTIMATES AND \\ AVERAGE INDIVIDUAL DOSE-COMMITMENT HISTOGRAM FOR}

SALEM 1 AND 2

Dose Commitments (person-rem) from Waterborne Pathways

Total Body GI-LII $\underline{\text { Thyroid Bone Liver }}$

$\begin{array}{llllll}\text { Infant } & 0.0 \mathrm{E}+00 & 0.0 \mathrm{E}+00 & 0.0 \mathrm{E}+00 & 0.0 \mathrm{E}+00 & 0.0 \mathrm{E}+00 \\ \text { Child } & 4.5 \mathrm{E}-02 & 7.0 \mathrm{E}-02 & 2.9 \mathrm{E}-02 & 1.5 \mathrm{E}-01 & 9.3 \mathrm{E}-02 \\ \text { Teen } & 3.1 \mathrm{E}-02 & 1.4 \mathrm{E}-01 & 2.1 \mathrm{E}-02 & 8.1 \mathrm{E}-02 & 7.2 \mathrm{E}-02 \\ \text { Adult } & 2.0 \mathrm{E}-01 & 1.2 \mathrm{E}+00 & 1.4 \mathrm{E}-01 & 4.8 \mathrm{E}-01 & 4.2 \mathrm{E}-01 \\ \text { TOTAL } & 2.8 \mathrm{E}-01 & 1.5 \mathrm{E}+00 & 1.9 \mathrm{E}-01 & 7.1 \mathrm{E}-01 & 5.8 \mathrm{E}-01\end{array}$

Dose Commitments (person-rem) from Airborne Pathways

Iotal Body GI-LII Ihyroid Bone Liver Lung

$\begin{array}{lllllll}\text { Infant } & 8.3 \mathrm{E}-03 & 8.3 \mathrm{E}-03 & 1.0 \mathrm{E}-02 & 1.4 \mathrm{E}-03 & 8.3 \mathrm{E}-03 & 8.4 \mathrm{E}-03 \\ \text { Child } & 1.2 \mathrm{E}-01 & 1.2 \mathrm{E}-01 & 1.3 \mathrm{E}-01 & 1.6 \mathrm{E}-02 & 1.2 \mathrm{E}-01 & 1.2 \mathrm{E}-01 \\ \text { Teen } & 8.1 \mathrm{E}-02 & 8.1 \mathrm{E}-02 & 8.6 \mathrm{E}-02 & 1.2 \mathrm{E}-02 & 8.1 \mathrm{E}-02 & 8.4 \mathrm{E}-02 \\ \text { Adult } & 4.5 \mathrm{E}-01 & 4.5 \mathrm{E}-01 & 4.7 \mathrm{E}-01 & 7.1 \mathrm{E}-02 & 4.5 \mathrm{E}-01 & 4.6 \mathrm{E}-01 \\ \text { TOTAL } & 6.6 \mathrm{E}-01 & 6.6 \mathrm{E}-01 & 6.9 \mathrm{E}-01 & 1.0 \mathrm{E}-01 & 6.6 \mathrm{E}-01 & 6.7 \mathrm{E}-01\end{array}$

Production/Consumption factors:

Produce: 0.070 Milk: 0.38 Meat: 0.055

Fraction of Population Receiving an Average Individual

Total-Body Dose Commitment from Airborne Pathways

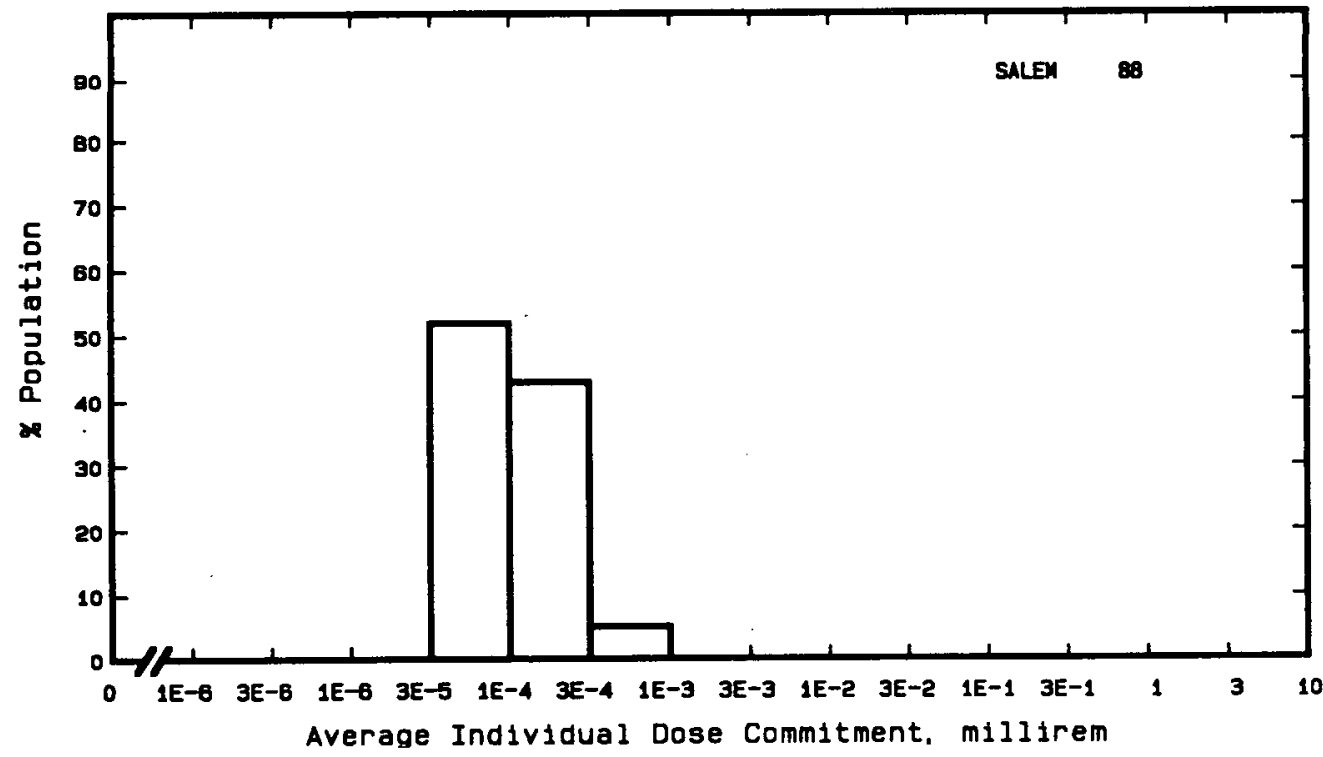


Site: SAN ONOFRE

CAMP PENDLETON, CALIFORNIA

Location: $\quad$ N $33.3703^{\circ} \quad$ W $117.5569^{\circ}$

POPULATION DATA

Total Population Within 2-to-80-km Region: 5.5E6

Major Metropolitan Centers Within Region:

Center

Population

Location

San Diego SMSA

$2,100,000$

Anaheim-Santa Ana-Garden Grove SMSA 2,100,000

Long Beach

Huntington Beach

410,000

180,000

Riverside

180,000

Pomona

110,000

$\begin{array}{lll}68 & \mathrm{~km} & \text { SSE } \\ 62 & \mathrm{~km} & \mathrm{NW} \\ 75 & \mathrm{~km} & \mathrm{NW} \\ 61 & \mathrm{~km} & \mathrm{NW} \\ 68 & \mathrm{~km} & \mathrm{~N} \\ 79 & \mathrm{~km} & \mathrm{NNW}\end{array}$

SITE-SPECIFIC DATA - AIRBORNE PATHWAYS

Average Annual State Production

of Crops and Animal Products

In $80-\mathrm{km}$ Radius Circle

Regional Productivity Factor:

Animal Grazing Factor:
Veg: $4.8 \mathrm{E} 7 \mathrm{kilogram}$

Milk: 2.3E8 liter

Meat: $5.0 \mathrm{E} 7$ kilogram

0.6

1

Meteorology Period of Record: 25 JAN 73 - 24 JAN 76 Recovery: 88\%

SITE-SPECIFIC DATA - WATERBORNE PATHWAYS via PACIFIC OCEAN

Average Dilution Flow

from all 3 Plants: $3,500 \mathrm{ft}^{3} / \mathrm{s}$

Fish:

Edible Harvest: $2.9 \mathrm{E} 4 \mathrm{~kg} / \mathrm{yr}$

Invertebrates:

Dilution Factor: 1 (a)

Edible Harvest: 2 .9E3 $\mathrm{kg} / \mathrm{yr}$
Dilution Factor: 1 (a)

(a) Seafood caught in undiluted effluent according to FES (1973). 
POPULATION DOSE-COMMITMENT ESTIMATES AND

AVERAGE INDIVIDUAL DOSE-COMMITMENT HISTOGRAM FOR

SAN ONOFRE 1,2 , AND 3

Dose Commitments (person-rem) from Waterborne Pathways

Total Body GI-LII Thyroid Bone Liver

$\begin{array}{llllll}\text { Infant } & 0.0 \mathrm{E}+00 & 0.0 \mathrm{E}+00 & 0.0 \mathrm{E}+00 & 0.0 \mathrm{E}+00 & 0.0 \mathrm{E}+00 \\ \text { Child } & 1.5 \mathrm{E}-03 & 3.8 \mathrm{E}-03 & 1.8 \mathrm{E}-02 & 4.6 \mathrm{E}-03 & 5.2 \mathrm{E}-03 \\ \text { Teen } & 2.0 \mathrm{E}-03 & 7.7 \mathrm{E}-03 & 1.3 \mathrm{E}-02 & 2.7 \mathrm{E}-03 & 4.4 \mathrm{E}-03 \\ \text { Adult } & 1.9 \mathrm{E}-02 & 6.7 \mathrm{E}-02 & 8.3 \mathrm{E}-02 & 1.6 \mathrm{E}-02 & 2.7 \mathrm{E}-02 \\ \text { TOTAL } & 2.3 \mathrm{E}-02 & 7.9 \mathrm{E}-02 & 1.1 \mathrm{E}-01 & 2.3 \mathrm{E}-02 & 3.6 \mathrm{E}-02\end{array}$

Dose Commitments (person-rem) from Airborne Pathways

Total Body GI-LII Thyroid Bone Liver $\underline{\text { Lung }}$

$\begin{array}{lllllll}\text { Infant } & 7.0 \mathrm{E}-03 & 6.8 \mathrm{E}-03 & 1.2 \mathrm{E}-01 & 6.9 \mathrm{E}-03 & 7.2 \mathrm{E}-03 & 7.3 \mathrm{E}-03 \\ \text { Child } & 7.8 \mathrm{E}-02 & 7.7 \mathrm{E}-02 & 6.8 \mathrm{E}-01 & 7.5 \mathrm{E}-02 & 7.9 \mathrm{E}-02 & 8.4 \mathrm{E}-02 \\ \text { Teen } & 5.7 \mathrm{E}-02 & 5.6 \mathrm{E}-02 & 3.0 \mathrm{E}-01 & 5.4 \mathrm{E}-02 & 5.7 \mathrm{E}-02 & 6.6 \mathrm{E}-02 \\ \text { Adult } & 3.4 \mathrm{E}-01 & 3.4 \mathrm{E}-01 & 1.2 \mathrm{E}+00 & 3.3 \mathrm{E}-01 & 3.4 \mathrm{E}-01 & 3.7 \mathrm{E}-01 \\ \text { TOTAL } & 4.8 \mathrm{E}-01 & 4.8 \mathrm{E}-01 & 2.3 \mathrm{E}+00 & 4.6 \mathrm{E}-01 & 4.9 \mathrm{E}-01 & 5.3 \mathrm{E}-01\end{array}$

Production/Consumption factors:

Produce: 0.027 Milk: $0.19 \quad$ Meat: 0.067

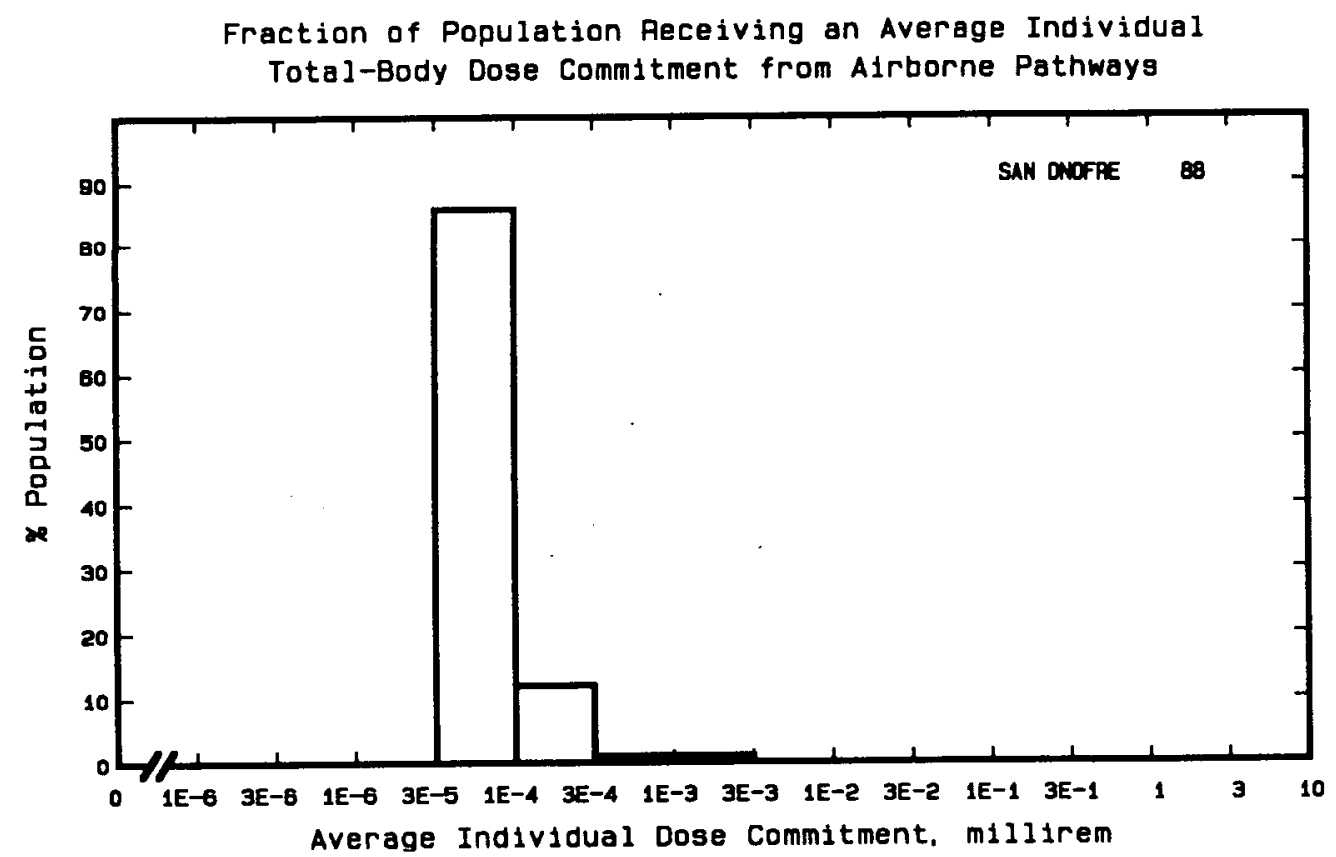


Site: SEQUOYAH

HAMILTON COUNTY, TENNESSEE

Location: $\quad$ N $35.2233^{\circ} \quad$ W $85.0878^{\circ}$

POPULATION DATA

Total Population Within 2-to-80-km Region: $8.8 E 5$

Major Metropolitan Centers Within Region:

Center

Chattanooga SMSA

Cleveland

East Ridge

Dalton

Athens
Population

440,000

26,000

21,000

21,000

12,000
Location

$28 \mathrm{~km} \quad \mathrm{SW}$

$21 \mathrm{~km} \quad \mathrm{SE}$

$27 \mathrm{~km} \quad \mathrm{SSW}$

$50 \mathrm{~km} \mathrm{~S}$

$53 \mathrm{~km}$ ENE

SITE-SPECIFIC DATA - AIRBORNE PATHWAYS

Average Annual State Production

of Crops and Animal Products

In 80-km Radius Circle

Regional Productivity Factor:

Animal Grazing Factor:
Veg: $1.1 \mathrm{E} 7 \mathrm{kilogram}$

Milk: 1.6E8 liter

Meat: $1.2 \mathrm{E} 8$ kilogram

0.25

0.7

Meteorology Period of Record: 1 JAN 72 - 31 DEC 72 Recovery: $93 \%$

\section{SITE-SPECIFIC DATA - WATERBORNE PATHWAYS via TENNESSEE RIVER}

Drinking Water:

Fish:
Average River Flow

at Site: $35,000 \mathrm{ft}^{3} / \mathrm{s}$

Exposed Population: 460,000

Dilution Factor: 1

Edible Harvest: $1.7 \mathrm{E5}$ (a) $\mathrm{kg} / \mathrm{yr}$

Dilution Factor: 1

(a) Catch data given in FES (1974). 
POPULATION DOSE-COMMITMENT ESTIMATES AND
AVERAGE INDIVIDUAL DOSE-COMMITMENT HISTOGRAM FOR

SEQUOYAH 1 AND 2

Dose Commitments (person-rem) from Waterborne Pathways

Total Body GI-LII Ihyroid Bone Liver

$\begin{array}{llllll}\text { Infant } & 2.7 \mathrm{E}-03 & 2.5 \mathrm{E}-03 & 3.0 \mathrm{E}-03 & 2.1 \mathrm{E}-03 & 4.7 \mathrm{E}-03 \\ \text { Child } & 3.5 \mathrm{E}-02 & 3.0 \mathrm{E}-02 & 3.0 \mathrm{E}-02 & 4.4 \mathrm{E}-02 & 6.9 \mathrm{E}-02 \\ \text { Teen } & 2.1 \mathrm{E}-02 & 1.4 \mathrm{E}-02 & 1.1 \mathrm{E}-02 & 1.8 \mathrm{E}-02 & 3.6 \mathrm{E}-02 \\ \text { Adult } & 2.0 \mathrm{E}-01 & 1.3 \mathrm{E}-01 & 9.2 \mathrm{E}-02 & 1.1 \mathrm{E}-01 & 2.4 \mathrm{E}-01 \\ \text { TOTAL } & 2.6 \mathrm{E}-01 & 1.7 \mathrm{E}-01 & 1.4 \mathrm{E}-01 & 1.7 \mathrm{E}-01 & 3.5 \mathrm{E}-01\end{array}$

Dose Commitments (person-rem) from Airborne Pathways

Total Body GI-LII Thyroid Bone Liver Lung

$\begin{array}{lllllll}\text { Infant } & 2.4 \mathrm{E}-04 & 2.4 \mathrm{E}-04 & 2.5 \mathrm{E}-04 & 1.6 \mathrm{E}-04 & 2.4 \mathrm{E}-04 & 2.6 \mathrm{E}-04 \\ \text { Child } & 3.1 \mathrm{E}-03 & 3.1 \mathrm{E}-03 & 3.1 \mathrm{E}-03 & 1.8 \mathrm{E}-03 & 3.1 \mathrm{E}-03 & 3.3 \mathrm{E}-03 \\ \text { Teen } & 2.3 \mathrm{E}-03 & 2.3 \mathrm{E}-03 & 2.3 \mathrm{E}-03 & 1.3 \mathrm{E}-03 & 2.3 \mathrm{E}-03 & 2.6 \mathrm{E}-03 \\ \text { Adult } & 1.4 \mathrm{E}-02 & 1.4 \mathrm{E}-02 & 1.4 \mathrm{E}-02 & 8.0 \mathrm{E}-03 & 1.4 \mathrm{E}-02 & 1.5 \mathrm{E}-02 \\ \text { TOTAL } & 1.9 \mathrm{E}-02 & 1.9 \mathrm{E}-02 & 2.0 \mathrm{E}-02 & 1.1 \mathrm{E}-02 & 1.9 \mathrm{E}-02 & 2.1 \mathrm{E}-02\end{array}$

Production/Consumption factors:

Produce: $0.016 \quad$ Milk: $0.36 \quad$ Meat: 0.42

Fraction of Population Receiving an Average Individual

Total-Body Dose Commitment from Airborne Pathways

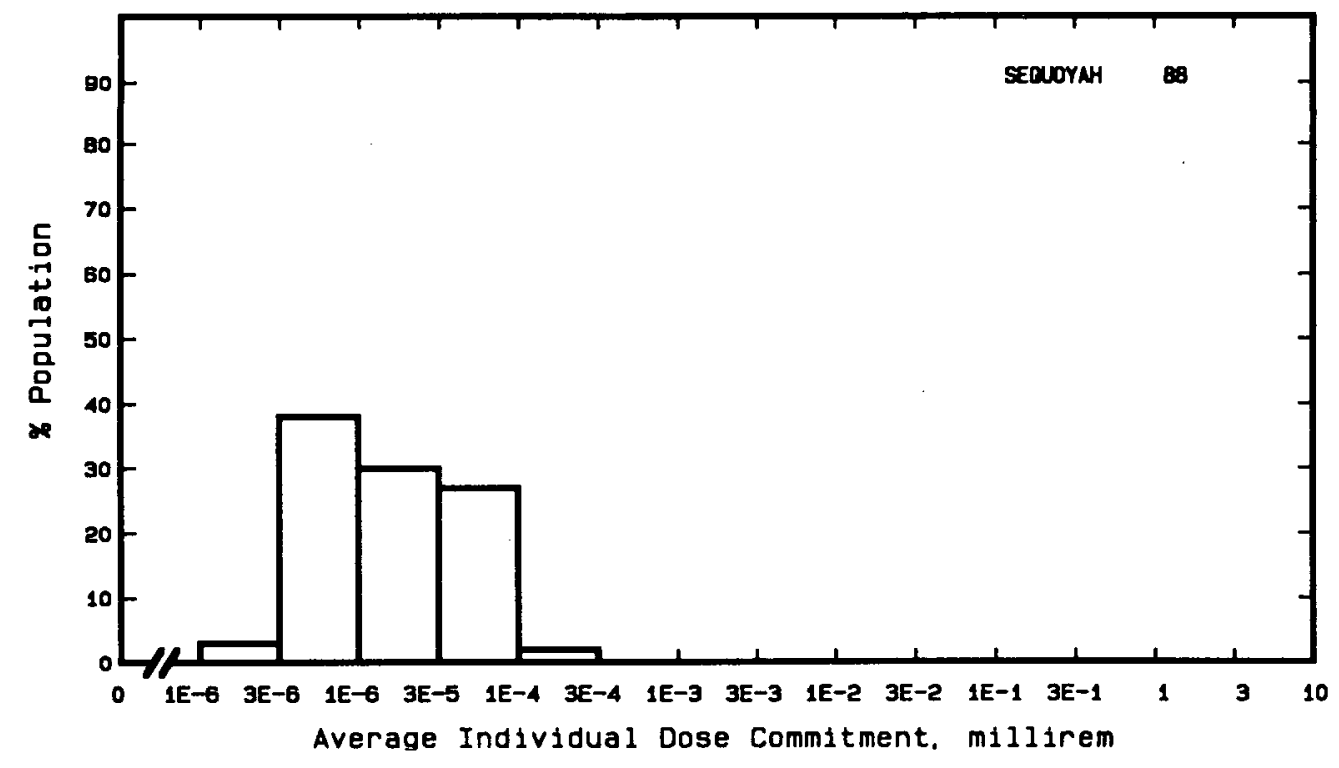


Site: SHOREHAM

BROOKHAVEN, NEW YORK

Location: $\quad N 40.9608^{\circ} \quad$ W $72.8658^{\circ}$

POPULATION DATA

Total Population Within 2-to-80-km Region: 5.2E6

Major Metropolitan Centers Within Region:

Center

Nassau county - Suffolk County SMSA

New Haven - West Haven SMSA

Bridgeport SMSA

Waterbury SMSA

Stamford SMSA

Norwalk

Meriden
Population

$2,600,000$

430,000

400,000

230,000

130,000

80,000

59,000
Location

$50 \mathrm{~km} \quad$ WSW

$38 \mathrm{~km} \quad \mathrm{NW}$

$37 \mathrm{~km} \quad \mathrm{NW}$

$68 \mathrm{~km} \quad \mathrm{NNW}$

$57 \mathrm{~km} \quad$ WNW

$26 \mathrm{~km} \quad W N W$

$68 \mathrm{~km} \mathrm{~N}$

SITE-SPECIFIC DATA - AIRBORNE PATHWAYS

Average Annual State Production

of Crops and Animal Products

In $80-\mathrm{km}$ Radius Circle

Regional Productivity Factor:

Animal Grazing Factor:
Veg: $7.6 E 7$ kilogram

Milk: 7.0E8 liter

Meat: $3.3 \mathrm{E} 7 \mathrm{kilogram}$

0.5

0.6

Meteorology Period of Record: 1 OCT 73 - 30 SEP 74 Recovery: $90 \%$

SITE-SPECIFIC DATA - WATERBORNE PATHWAYS via LONG ISLAND SOUND

Average Dilution Flow

from Plant: $1.0 \mathrm{ft}^{3} / \mathrm{s}$

Fish:

Edible Harvest: $2.955 \mathrm{~kg} / \mathrm{yr}$

Dilution Factor: 0.001

Invertebrates:

Edible Harvest: $3.6 \mathrm{E} 5 \mathrm{~kg} / \mathrm{yr}$

Dilution Factor: 0.1 


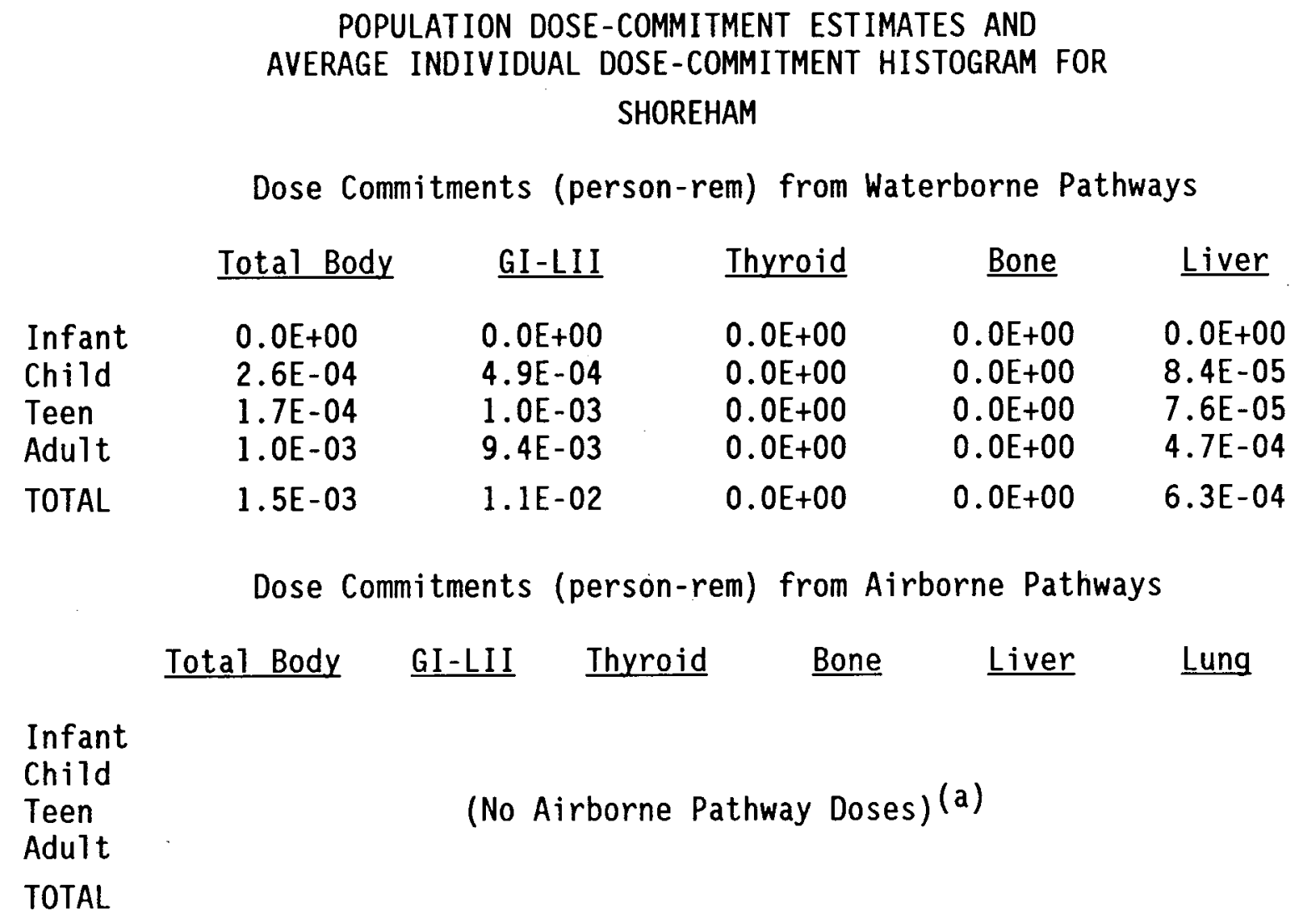

(a) No atmospheric releases reported (Tichler, et al. 1991). 
Site: SOUTH TEXAS

PALACIOS, TEXAS

Location: $\quad$ N $28.7000^{\circ} \quad$ W $96.2133^{\circ}$

POPULATION DATA

Total Population Within 2-to-80-km Region: 8.9E5

Major Metropolitan Centers Within Region:

Center

Bay City Lake Jackson

Freeport

Angleton
Population

21,000

23,000

16,000

16,000
Location

$19 \mathrm{~km} \quad \mathrm{NNE}$

$67 \mathrm{~km} \quad \mathrm{ENE}$

$72 \mathrm{~km}$ ENE

$74 \mathrm{~km} \quad \mathrm{NE}$

SITE-SPECIFIC DATA - AIRBORNE PATHWAYS

Average Annual State Production

of Crops and Animal Products

In $80-\mathrm{km}$ Radius Circle

Regional Productivity Factor:

Animal Grazing Factor:
Veg: $\quad 2.3 E 7$ kilogram

Milk: 4.3E7 liter

Meat: $1.1 \mathrm{E} 8$ kilogram

Meteorology Period of Record: 1 JAN $89-31$ DEC 89 Recovery: N/A

SITE-SPECIFIC DATA - WATERBORNE PATHWAYS via COLORADO RIVER

Average River Flow at Site: $800 \mathrm{ft}^{3} / \mathrm{s}$

Drinking Water:

Exposed Population:

Dilution Factor: 1

Fish:

Edible Harvest: $0.18 \mathrm{~kg} / \mathrm{yr}(\mathrm{a})$

Dilution Factor: 1

Invertebrates:

Edible Harvest: $0.91 \mathrm{~kg} / \mathrm{yr}(\mathrm{b})$

Dilution Factor: 1

(a) Ten percent of population assumed to eat $5 \mathrm{~g} / \mathrm{d}$ of fish used in lieu of catch data.

(b) Average individual consumption rate of $2.5 \mathrm{~g} / \mathrm{d}$ as given in the FES (1972) used in lieu of catch data. 


\section{POPULATION DOSE-COMMITMENT ESTIMATES AND AVERAGE INDIVIDUAL DOSE-COMMITMENT HISTOGRAM FOR \\ SOUTH TEXAS}

Dose Commitments (person-rem) from Waterborne Pathways

Iotal Body GI-LII Thyroid Bone Liver

$\begin{array}{llllll}\text { Infant } & 0.0 \mathrm{E}+00 & 0.0 \mathrm{E}+00 & 0.0 \mathrm{E}+00 & 0.0 \mathrm{E}+00 & 0.0 \mathrm{E}+00 \\ \text { Child } & 2.3 \mathrm{E}-02 & 5.2 \mathrm{E}-02 & 1.8 \mathrm{E}-02 & 7.0 \mathrm{E}-03 & 1.8 \mathrm{E}-02 \\ \text { Teen } & 1.6 \mathrm{E}-02 & 1.1 \mathrm{E}-01 & 1.2 \mathrm{E}-02 & 4.2 \mathrm{E}-03 & 1.6 \mathrm{E}-02 \\ \text { Adult } & 9.7 \mathrm{E}-02 & 9.5 \mathrm{E}-01 & 8.3 \mathrm{E}-02 & 2.5 \mathrm{E}-02 & 9.7 \mathrm{E}-02 \\ \text { TOTAL } & 1.4 \mathrm{E}-01 & 1.1 \mathrm{E}+00 & 1.1 \mathrm{E}-01 & 3.6 \mathrm{E}-02 & 1.3 \mathrm{E}-01\end{array}$

Dose Commitments (person-rem) from Airborne Pathways

Total Body GI-LII Thyroid Bone Liver Lung

$\begin{array}{lllllll}\text { Infant } & 4.0 \mathrm{E}-04 & 4.0 \mathrm{E}-04 & 5.0 \mathrm{E}-04 & 3.9 \mathrm{E}-04 & 4.0 \mathrm{E}-04 & 4.0 \mathrm{E}-04 \\ \text { Child } & 4.5 \mathrm{E}-03 & 4.5 \mathrm{E}-03 & 5.1 \mathrm{E}-03 & 4.3 \mathrm{E}-03 & 4.5 \mathrm{E}-03 & 4.5 \mathrm{E}-03 \\ \text { Teen } & 3.3 \mathrm{E}-03 & 3.3 \mathrm{E}-03 & 3.5 \mathrm{E}-03 & 3.2 \mathrm{E}-03 & 3.3 \mathrm{E}-03 & 3.3 \mathrm{E}-03 \\ \text { Adult } & 2.0 \mathrm{E}-02 & 2.0 \mathrm{E}-02 & 2.1 \mathrm{E}-02 & 1.9 \mathrm{E}-02 & 2.0 \mathrm{E}-02 & 2.0 \mathrm{E}-02 \\ \text { TOTAL } & 2.8 \mathrm{E}-02 & 2.8 \mathrm{E}-02 & 3.0 \mathrm{E}-02 & 2.7 \mathrm{E}-02 & 2.8 \mathrm{E}-02 & 2.8 \mathrm{E}-02\end{array}$

Production/Consumption factors:

Produce: $0.17 \quad$ Milk: $0.48 \quad$ Meat: 1.9

Fraction of Population Aeceiving an Average Individual

Total-Body Dose Commitment from Alrborne Pathways

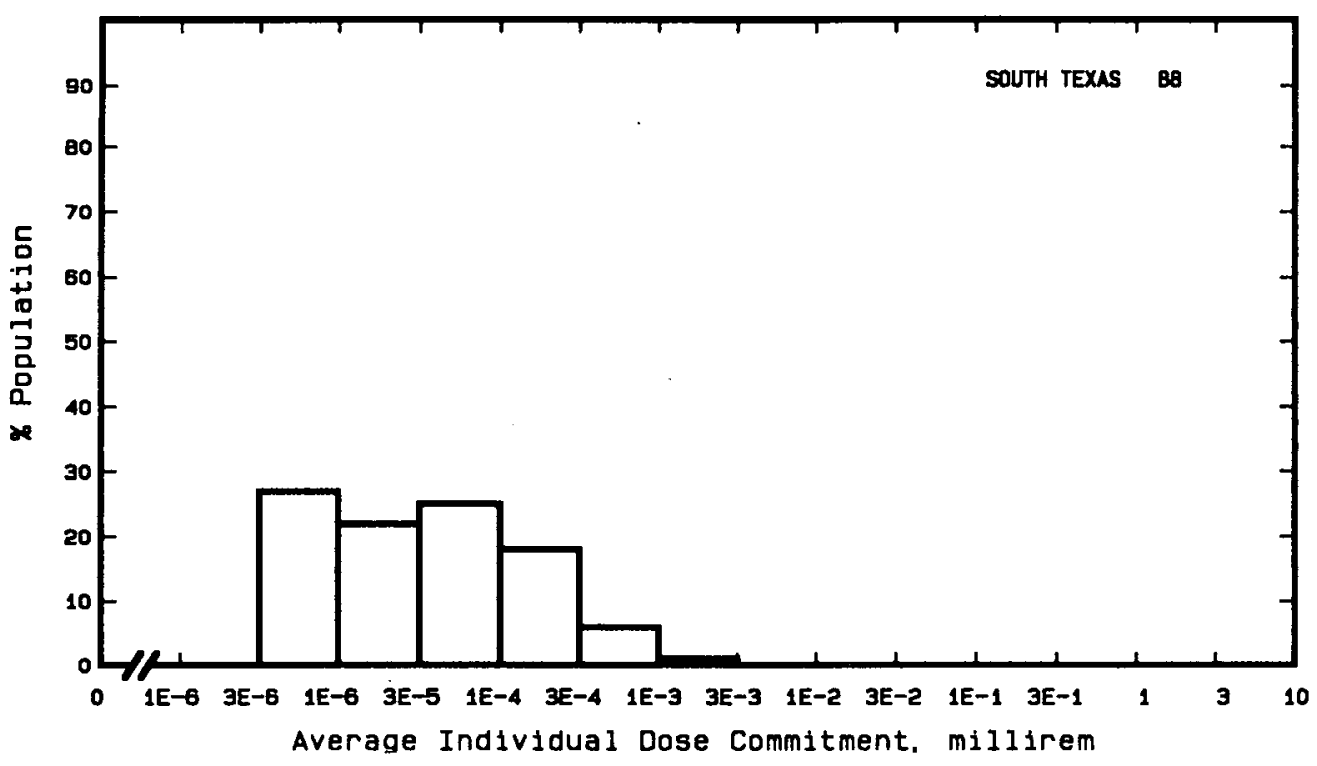


Site: SUMMER

JENKINSVILLE, SOUTH CAROLINA

Location: N $34.2958^{\circ} \quad$ W $81.3203^{\circ}$

POPULATION DATA

Total Population Within 2-to-80-km Region: 9.0 E5

Major Metropolitan Centers Within Region:

Center

Columbia SMSA

Rock Hill

Greenwood

Union

Laurens
Population

460,000

36,000

23,000

11,000

11,000

\section{Location}

$42 \mathrm{~km} \quad \mathrm{SE}$

$75 \mathrm{~km}$ NNE

$78 \mathrm{~km} \quad W$

$54 \mathrm{~km} \quad \mathrm{NNW}$

$68 \mathrm{~km}$ WNW

SITE-SPECIFIC DATA - AIRBORNE PATHWAYS

Average Annual State Production

Veg: $\quad 7.4 \mathrm{E} 6 \mathrm{ki}$ logram

of Crops and Animal Products

Milk: $5.7 E 7$ liter

In 80-km Radius Circle Meat: $5.0 \mathrm{E} 7 \mathrm{kilogram}$

Regional Productivity Factor:

Animal Grazing Factor:

0.9

0.7

Meteorology Period of Record: 1 Jan $75-31$ Dec $75 \quad$ Recovery: $98 \%$

SITE-SPECIFIC DATA - WATERBORNE PATHWAYS via PARR RES, and BROAD RIVER

Average River Flow

at Site: $6,000 \mathrm{ft}^{3} / \mathrm{s}$

Drinking Water:

Exposed Population: 130,000

Dilution Factor: 1

Fish:

Edible Harvest: (a)

Dilution Factor: 1

(a) Average individual consumption rate of $2.2 \mathrm{~kg} / \mathrm{yr}$ as given in the FES (1973) used in lieu of catch data. 
POPULATION DOSE-COMMITMENT ESTIMATES AND

AVERAGE INDIVIDUAL DOSE-COMMITMENT HISTOGRAM FOR

SUMMER

Dose Commitments (person-rem) from Waterborne Pathways

Total Body GI-LII Thyroid Bone Liver

$\begin{array}{llllll}\text { Infant } & 1.5 \mathrm{E}-02 & 1.5 \mathrm{E}-02 & 5.3 \mathrm{E}-02 & 4.8 \mathrm{E}-03 & 2.1 \mathrm{E}-02 \\ \text { Child } & 7.0 \mathrm{E}-01 & 2.7 \mathrm{E}-01 & 4.8 \mathrm{E}-01 & 2.1 \mathrm{E}+00 & 3.0 \mathrm{E}+00 \\ \text { Teen } & 1.1 \mathrm{E}+00 & 2.8 \mathrm{E}-01 & 1.8 \mathrm{E}-01 & 1.3 \mathrm{E}+00 & 2.4 \mathrm{E}+00 \\ \text { Adult } & 1.1 \mathrm{E}+01 & 2.5 \mathrm{E}+00 & 1.3 \mathrm{E}+00 & 7.5 \mathrm{E}+00 & 1.5 \mathrm{E}+01 \\ \text { TOTAL } & 1.3 \mathrm{E}+01 & 3.0 \mathrm{E}+00 & 2.0 \mathrm{E}+00 & 1.1 \mathrm{E}+01 & 2.0 \mathrm{E}+01\end{array}$

Dose Commitments (person-rem) from Airborne Pathways

Total Body GI-LII Thyroid Bone Liver Lung

$\begin{array}{lllllll}\text { Infant } & 1.0 \mathrm{E}-04 & 9.9 \mathrm{E}-05 & 7.3 \mathrm{E}-04 & 9.7 \mathrm{E}-05 & 1.0 \mathrm{E}-04 & 1.1 \mathrm{E}-04 \\ \text { Child } & 1.1 \mathrm{E}-03 & 1.1 \mathrm{E}-03 & 4.9 \mathrm{E}-03 & 1.1 \mathrm{E}-03 & 1.1 \mathrm{E}-03 & 1.2 \mathrm{E}-03 \\ \text { Teen } & 8.2 \mathrm{E}-04 & 8.2 \mathrm{E}-04 & 2.5 \mathrm{E}-03 & 7.8 \mathrm{E}-04 & 8.3 \mathrm{E}-04 & 9.6 \mathrm{E}-04 \\ \text { Adult } & 5.0 \mathrm{E}-03 & 5.0 \mathrm{E}-03 & 1.1 \mathrm{E}-02 & 4.7 \mathrm{E}-03 & 5.0 \mathrm{E}-03 & 5.4 \mathrm{E}-03 \\ \text { TOTAL } & 7.0 \mathrm{E}-03 & 7.0 \mathrm{E}-03 & 1.9 \mathrm{E}-02 & 6.6 \mathrm{E}-03 & 7.1 \mathrm{E}-03 & 7.7 \mathrm{E}-03\end{array}$

Production/Consumption factors:

Produce: $0.038 \quad$ Milk: $0.44 \quad$ Meat: 0.62

Fraction of Population Receiving an Average Individual Total-Body Dose Commitment from Airborne Pathways

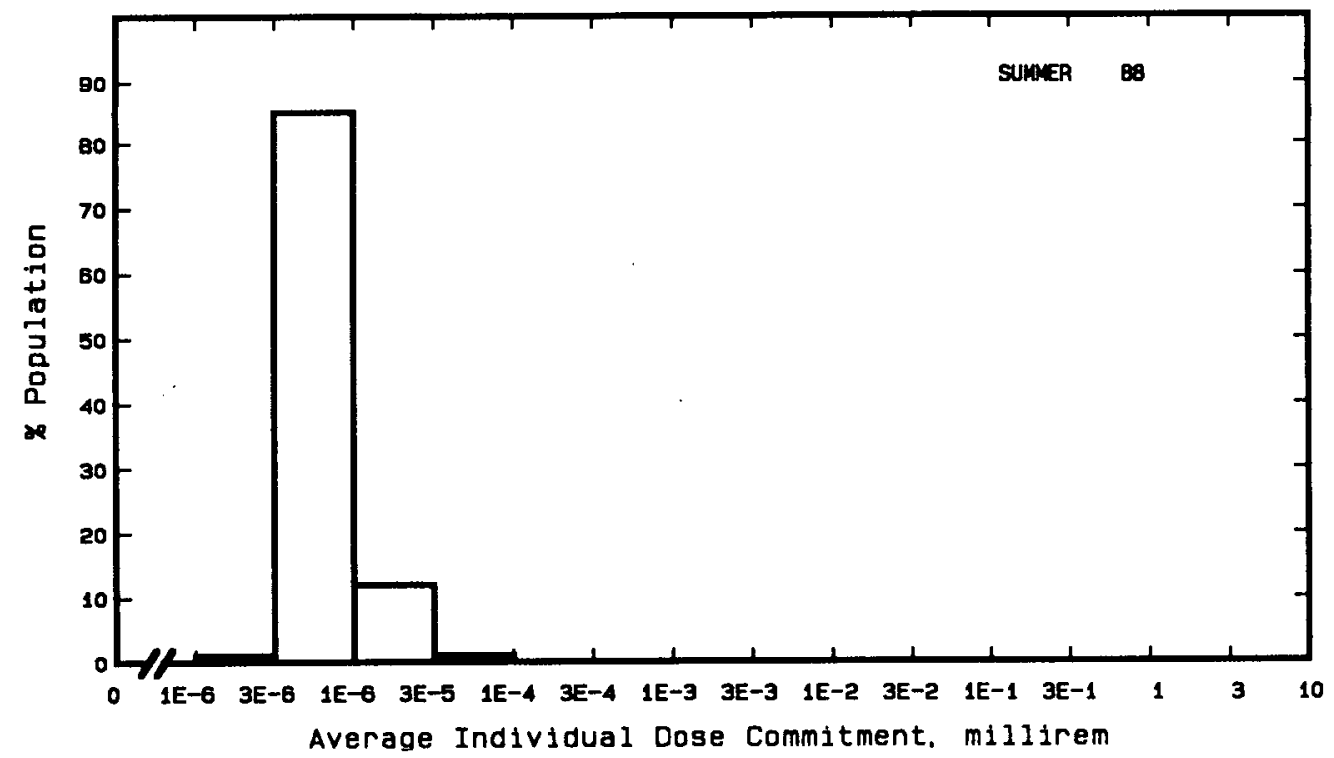


Site: SURRY

SURRY COUNTY, VIRGINIA

Location: $\quad$ N $37.1656^{\circ}$ W $76.6983^{\circ}$

POPULATION DATA

Total Population Within 2-to-80-km Region: 1.9E6

Major Metropolitan Centers Within Region:

Center

Norfolk-Virginia Beach-Portsmouth SMSA Richmond SMSA

Newport News-Hampton SMSA

Petersburg-Colonial Heights-Hopewe11 SMSA

Willi amsburg
Population

850,000

670,000

380,000

130,000

10,000
Location

$50 \mathrm{~km} \quad \mathrm{SE}$

$77 \mathrm{~km}$ WNW

$33 \mathrm{~km} \quad$ ESE

$63 \mathrm{~km} \quad \mathrm{~W}$

$12 \mathrm{~km} \mathrm{~N}$

SITE-SPECIFIC DATA - AIRBORNE PATHWAYS

Average Annual State Production

of Crops and Animal Products

In $80-\mathrm{km}$ Radius Circle

Regional Productivity Factor:

Animal Grazing Factor:
Veg: $3.5 \mathrm{E} 7 \mathrm{kilogram}$

Milk: $1.5 \mathrm{E} 8$ liter

Meat: 7.4E7 kilogram

0.8

0.7

Meteorology Period of Record: 3 MAR 74 - 2 MAR 75 Recovery: $91 \%$

Fish:

Invertebrates: (b)
Average River Flow

$$
\text { at Site: } 25,000 \mathrm{ft}^{3} / \mathrm{s}(\mathrm{a})
$$

Edible Harvest: $6.0 \mathrm{E} 5 \mathrm{~kg} / \mathrm{yr}$

Dilution Factor: 1

Edible Harvest: $1.1 \mathrm{E} 6 \mathrm{~kg} / \mathrm{yr}$

Dilution Factor: 1

(a) Flow includes fresh water river flow and saline "mixing flow" of estuary as given in FES (1972).

(b) Environment primarily salt water so invertebrates considered in lieu of drinking water. 


\section{POPULATION DOSE-COMMITMENT ESTIMATES AND \\ AVERAGE INDIVIDUAL DOSE-COMMITMENT HISTOGRAM FOR \\ SURRY 1 AND 2}

Dose Commitments (person-rem) from Waterborne Pathways

Total Body GI-LII Ihyroid Bone Liver

$\begin{array}{llllll}\text { Infant } & 0.0 \mathrm{E}+00 & 0.0 \mathrm{E}+00 & 0.0 \mathrm{E}+00 & 0.0 \mathrm{E}+00 & 0.0 \mathrm{E}+00 \\ \text { Child } & 7.4 \mathrm{E}-02 & 9.7 \mathrm{E}-02 & 4.5 \mathrm{E}-02 & 2.5 \mathrm{E}-01 & 1.6 \mathrm{E}-01 \\ \text { Teen } & 5.1 \mathrm{E}-02 & 1.9 \mathrm{E}-01 & 3.1 \mathrm{E}-02 & 1.4 \mathrm{E}-01 & 1.3 \mathrm{E}-01 \\ \text { Adult } & 3.5 \mathrm{E}-01 & 1.7 \mathrm{E}+00 & 2.1 \mathrm{E}-01 & 8.1 \mathrm{E}-01 & 7.3 \mathrm{E}-01 \\ \text { TOTAL } & 4.7 \mathrm{E}-01 & 1.9 \mathrm{E}+00 & 2.8 \mathrm{E}-01 & 1.2 \mathrm{E}+00 & 1.0 \mathrm{E}+00\end{array}$

Dose Commitments (person-rem) from Airborne Pathways

Total Body GI-LII Thyroid Bone Liver Lung

$\begin{array}{lllllll}\text { Infant } & 5.2 \mathrm{E}-04 & 4.9 \mathrm{E}-04 & 7.3 \mathrm{E}-03 & 5.0 \mathrm{E}-04 & 7.9 \mathrm{E}-04 & 6.0 \mathrm{E}-04 \\ \text { Child } & 6.7 \mathrm{E}-03 & 6.4 \mathrm{E}-03 & 4.8 \mathrm{E}-02 & 5.0 \mathrm{E}-03 & 8.5 \mathrm{E}-03 & 7.9 \mathrm{E}-03 \\ \text { Teen } & 4.8 \mathrm{E}-03 & 4.6 \mathrm{E}-03 & 2.2 \mathrm{E}-02 & 2.9 \mathrm{E}-03 & 5.4 \mathrm{E}-03 & 5.9 \mathrm{E}-03 \\ \text { Adult } & 2.8 \mathrm{E}-02 & 2.7 \mathrm{E}-02 & 9.1 \mathrm{E}-02 & 1.5 \mathrm{E}-02 & 2.9 \mathrm{E}-02 & 3.2 \mathrm{E}-02 \\ \text { TOTAL } & 4.0 \mathrm{E}-02 & 3.8 \mathrm{E}-02 & 1.7 \mathrm{E}-01 & 2.4 \mathrm{E}-02 & 4.4 \mathrm{E}-02 & 4.6 \mathrm{E}-02\end{array}$

Production/Consumption factors:

Produce: $0.076 \quad$ Milk: $0.48 \quad$ Meat: 0.39

Fraction of Population Receiving an Average Individual

Total-Body Dose Commitment from Alrborne Pathways

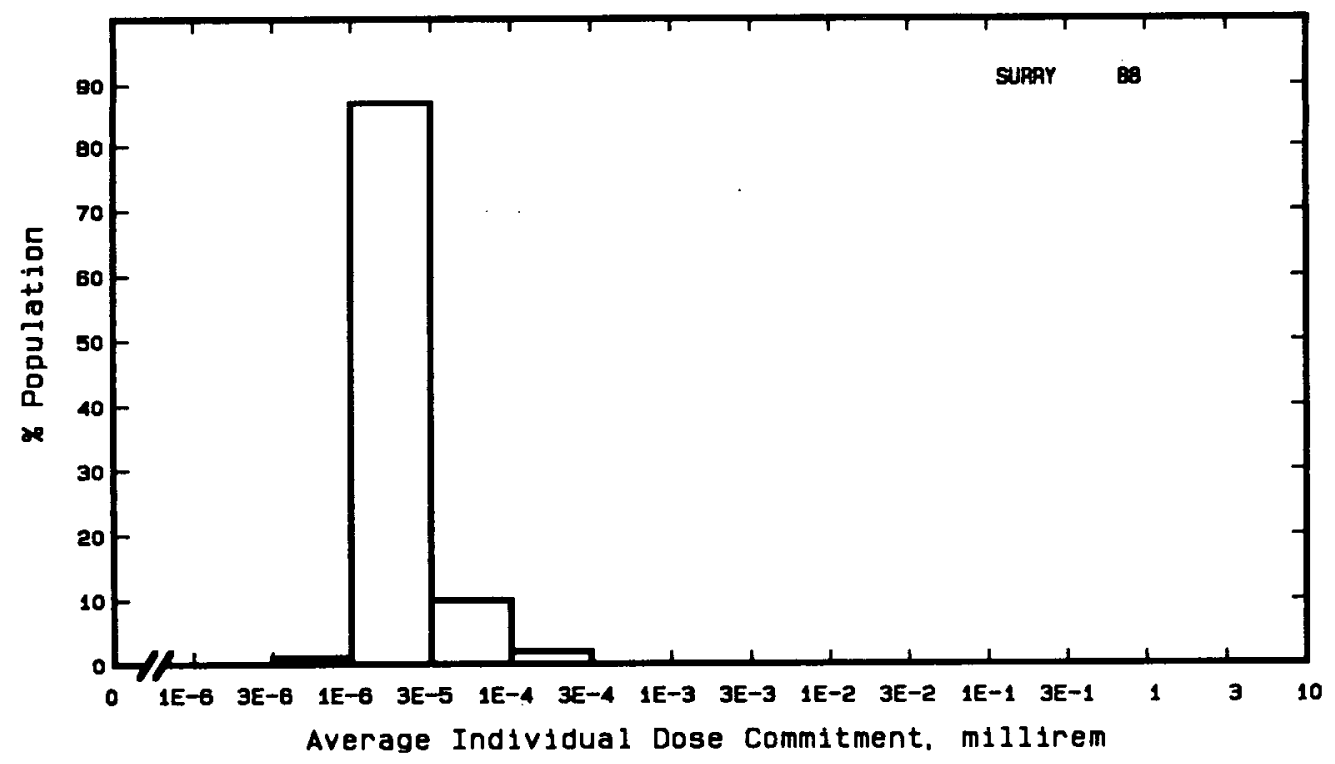


Site: SUSQUEHANNA

Location: $\quad$ N $41.1000^{\circ} \quad$ W $76.1500^{\circ}$

POPULATION DATA

Total Population Within 2-to-80-km Region: 1.5E6

Major Metropolitan Centers Within Region:

Center

Williamsport SMSA

Allentown

Scranton

Wilkes-Barre

Hazleton
Population

118,000

100,000

88,000

52,000

27,000

\section{Location}

$73 \mathrm{~km} \quad$ WNW

$79 \mathrm{~km} \quad \mathrm{SE}$

$53 \mathrm{~km} \quad \mathrm{NE}$

$28 \mathrm{~km} \quad \mathrm{NE}$

$21 \mathrm{~km} \quad \mathrm{SE}$

SITE-SPECIFIC DATA - AIRBORNE PATHWAYS

Average Annual State Production

of Crops and Animal Products

In $80-\mathrm{km}$ Radius Circle

Regional Productivity Factor:

Animal Grazing Factor:
Veg: $\quad 5.3 \mathrm{E} 7 \mathrm{kilogram}$

Milk: 5.3E8 liter

Meat: $5.4 \mathrm{E} 7 \mathrm{kilogram}$

Meteorology Period of Record: 1 Jan 75 - 31 Dec 75 Recovery: 98\%

SITE-SPECIFIC DATA - WATERBORNE PATHWAYS via SUSQUEHANNA RIVER

Average River Flow

at Site: $13,000 \mathrm{ft}^{3} / \mathrm{s}$

Drinking Water:

Exposed Population: 100,000

Dilution Factor: 1

Fish:

Edible Harvest: (a)

Dilution Factor: 0.1 (b)

(a) No fish catch data given in FES (1973), so generic consumption rates used (Table A.1).

(b) Ten percent of population consumes fish from river. 
POPULATION DOSE-COMMITMENT ESTIMATES AND

AVERAGE INDIVIDUAL DOSE-COMMITMENT HISTOGRAM FOR

\section{SUSQUEHANNA 1 AND 2}

Dose Commitments (person-rem) from Waterborne Pathways

Total Body GI-LII Thyroid Bone Liver

$\begin{array}{llllll}\text { Infant } & 1.0 \mathrm{E}-04 & 1.0 \mathrm{E}-04 & 9.3 \mathrm{E}-05 & 1.2 \mathrm{E}-05 & 1.2 \mathrm{E}-04 \\ \text { Child } & 1.9 \mathrm{E}-03 & 1.7 \mathrm{E}-03 & 1.1 \mathrm{E}-03 & 2.3 \mathrm{E}-03 & 4.5 \mathrm{E}-03 \\ \text { Teen } & 1.6 \mathrm{E}-03 & 1.6 \mathrm{E}-03 & 4.1 \mathrm{E}-04 & 1.4 \mathrm{E}-03 & 3.3 \mathrm{E}-03 \\ \text { Adult } & 1.5 \mathrm{E}-02 & 1.5 \mathrm{E}-02 & 3.5 \mathrm{E}-03 & 8.0 \mathrm{E}-03 & 2.1 \mathrm{E}-02 \\ \text { TOTAL } & 1.8 \mathrm{E}-02 & 1.8 \mathrm{E}-02 & 5.1 \mathrm{E}-03 & 1.2 \mathrm{E}-02 & 2.9 \mathrm{E}-02\end{array}$

Dose Commitments (person-rem) from Airborne Pathways

Total Body GI-LII Thyroid Bone Liver Lung

$\begin{array}{lllllll}\text { Infant } & 2.3 \mathrm{E}-04 & 2.2 \mathrm{E}-04 & 1.2 \mathrm{E}-03 & 4.4 \mathrm{E}-05 & 2.3 \mathrm{E}-04 & 2.3 \mathrm{E}-04 \\ \text { Child } & 3.0 \mathrm{E}-03 & 3.0 \mathrm{E}-03 & 8.7 \mathrm{E}-03 & 4.7 \mathrm{E}-04 & 3.0 \mathrm{E}-03 & 3.1 \mathrm{E}-03 \\ \text { Teen } & 2.0 \mathrm{E}-03 & 2.0 \mathrm{E}-03 & 4.2 \mathrm{E}-03 & 3.4 \mathrm{E}-04 & 2.0 \mathrm{E}-03 & 2.1 \mathrm{E}-03 \\ \text { Adult } & 1.1 \mathrm{E}-02 & 1.1 \mathrm{E}-02 & 1.8 \mathrm{E}-02 & 2.0 \mathrm{E}-03 & 1.1 \mathrm{E}-02 & 1.1 \mathrm{E}-02 \\ \text { TOTAL } & 1.6 \mathrm{E}-02 & 1.6 \mathrm{E}-02 & 3.2 \mathrm{E}-02 & 2.9 \mathrm{E}-03 & 1.6 \mathrm{E}-02 & 1.7 \mathrm{E}-02\end{array}$

Production/Consumption factors:

Produce: $0.16 \quad$ Milk: $2.4 \quad$ Meat: 0.41

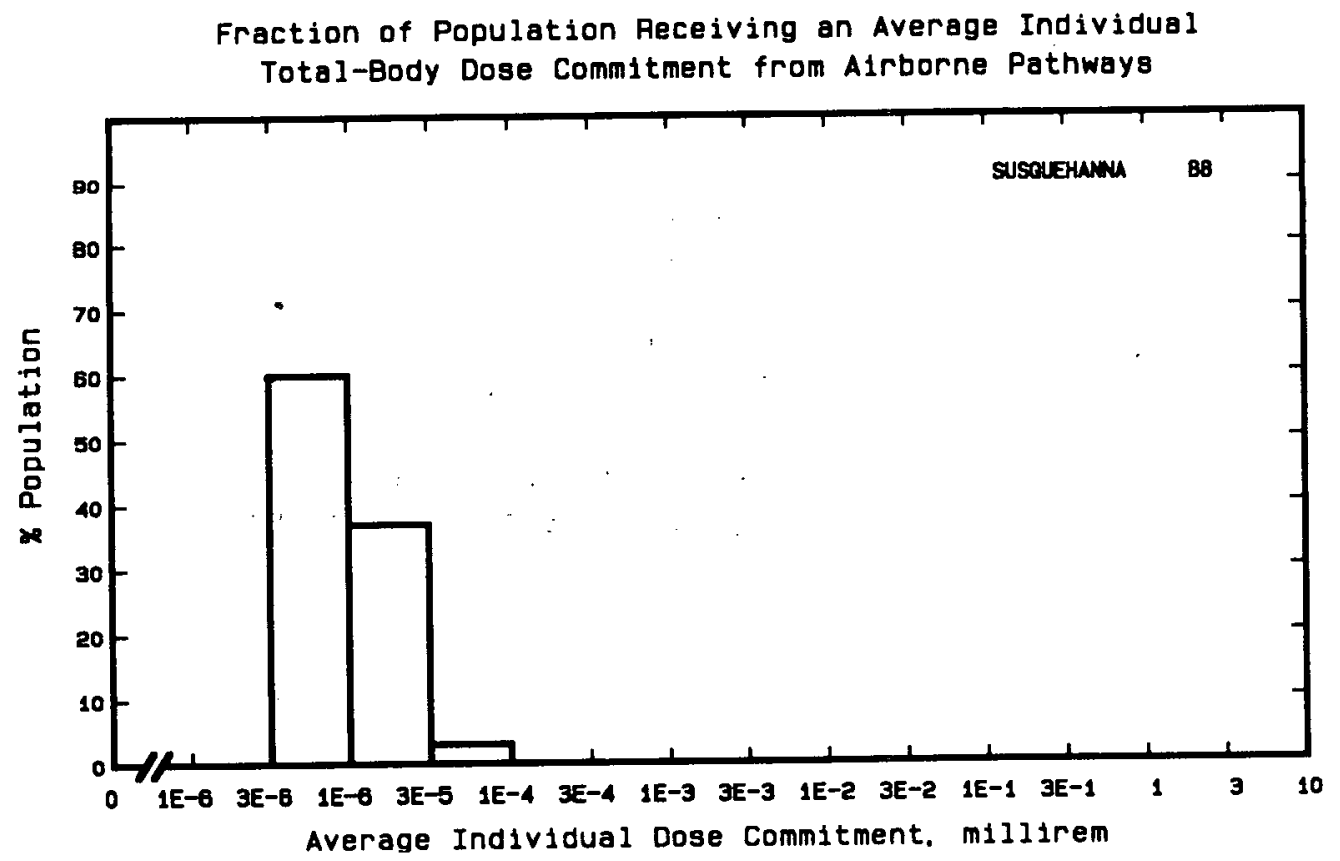


Site: THREE MILE ISLAND

THREE MILE ISLAND, PENNSYLVANIA

Location: $\quad$ N $40.1531^{\circ}$ W $76.7250^{\circ}$

POPULATION DATA

Total Population Within 2-to-80-km Region: 2.1E6

Major Metropolitan Centers Within Region:

Center

Harrisburg SMSA

York SMSA

Lancaster SMSA

Reading SMSA

Lebanon
Population

460,000

390,000

390,000

320,000

26,000
Location

$18 \mathrm{~km} \quad \mathrm{NW}$

$21 \mathrm{~km} \mathrm{~S}$

$38 \mathrm{~km} \quad$ ESE

$71 \mathrm{~km} \quad$ ENE

$33 \mathrm{~km} \quad \mathrm{NE}$

SITE-SPECIFIC DATA - AIRBORNE PATHWAYS

Average Annual State Production

of Crops and Animal Products

In 80-km Radius Circle

Regional Productivity Factor:

Animal Grazing Factor:
Veg: $5.3 \mathrm{E} 7 \mathrm{kilogram}$

Milk: 5.3E8 liter

Meat: 5.4E7 kilogram

1

0.5

Meteorology Period of Record: 1 OCT 72 - 30 SEP 73 Recovery: $80 \%$

SITE-SPECIFIC DATA - WATERBORNE PATHWAYS via SUSQUEHANNA RIVER

Average River Flow

at Site: $34,000 \mathrm{ft}^{3} / \mathrm{s}$

Drinking Water:

Exposed Population: 230,000

Dilution Factor: 1

Fish:

Edible Harvest: (a)

Dilution Factor: 0.025 (b)

(a) No fish catch data given in FES (1972), so generic consumption rates used (Table A-1).

(b) Ten percent of population obtain $25 \%$ of their fish from river according to FES. 
POPULATION DOSE-COMMITMENT ESTIMATES AND AVERAGE INDIVIDUAL DOSE-COMMITMENT HISTOGRAM FOR

THREE MILE ISLAND 1 AND 2

Dose Commitments (person-rem) from Waterborne Pathways (a)

Total Body GI-LII $\underline{\text { Thyroid }}$ Bone Liver

\begin{tabular}{|c|c|c|c|c|c|c|}
\hline $\begin{array}{l}\text { Infant } \\
\text { Child } \\
\text { Teen } \\
\text { Adult }\end{array}$ & $\begin{array}{l}1.8 \mathrm{E}-03 \\
2.1 \mathrm{E}-02 \\
9.3 \mathrm{E}-03 \\
8.3 \mathrm{E}-02\end{array}$ & $\begin{array}{l}1.8 \\
2.8 \\
7.9 \\
6.9\end{array}$ & & $\begin{array}{l}2.2 \mathrm{E}-03 \\
2.3 \mathrm{E}-02 \\
8.5 \mathrm{E}-03 \\
7.1 \mathrm{E}-02\end{array}$ & $\begin{array}{l}3.0 \mathrm{E}-04 \\
8.0 \mathrm{E}-03 \\
3.7 \mathrm{E}-03 \\
2.4 \mathrm{E}-02\end{array}$ & $\begin{array}{l}1.9 \mathrm{E}-03 \\
2.5 \mathrm{E}-02 \\
1.1 \mathrm{E}-02 \\
8.7 \mathrm{E}-02\end{array}$ \\
\hline \multirow[t]{3}{*}{ TOTAL } & $1.2 E-01$ & 9.7 & & $1.0 \mathrm{E}-01$ & $3.6 \mathrm{E}-02$ & $1.2 \mathrm{E}-01$ \\
\hline & \multicolumn{4}{|c|}{ Dose Commitments (person-rem) } & \multicolumn{2}{|c|}{ Pathways (a) } \\
\hline & Total Body & GI-LII & Thyroid & Bor & Liver & Lung \\
\hline $\begin{array}{l}\text { Infant } \\
\text { Child } \\
\text { Teen } \\
\text { Adult }\end{array}$ & $\begin{array}{l}3.2 \mathrm{E}-03 \\
3.6 \mathrm{E}-02 \\
2.6 \mathrm{E}-02 \\
1.6 \mathrm{E}-01\end{array}$ & $\begin{array}{l}3.2 \mathrm{E}-03 \\
3.6 \mathrm{E}-02 \\
2.6 \mathrm{E}-02 \\
1.6 \mathrm{E}-01\end{array}$ & $\begin{array}{l}4.8 \mathrm{E}-03 \\
4.7 \mathrm{E}-02 \\
3.1 \mathrm{E}-02 \\
1.7 \mathrm{E}-01\end{array}$ & $\begin{array}{l}2.8 \mathrm{E} \\
3.1 \mathrm{E} \\
2.3 \mathrm{E} \\
1.4 \mathrm{E}\end{array}$ & $\begin{array}{l}3.2 \mathrm{E}-03 \\
3.6 \mathrm{E}-02 \\
2.6 \mathrm{E}-02 \\
1.6 \mathrm{E}-01\end{array}$ & $\begin{array}{l}3.4 \mathrm{E}-03 \\
4.0 \mathrm{E}-02 \\
3.1 \mathrm{E}-02 \\
1.7 \mathrm{E}-01\end{array}$ \\
\hline TOTAL. & $2.2 \mathrm{E}-01$ & 2. $2 \mathrm{E}-01$ & $2.6 \mathrm{E}-01$ & $1.9 \mathrm{E}$ & $2.2 E-01$ & $2.5 \mathrm{E}-01$ \\
\hline
\end{tabular}

Production/Consumption factors:

Produce: 0.13 Milk: 1.9 Meat: 0.32

Fraction of Population Receiving an Average Individual Total-Body Dose Commitment from Airborne Pathways

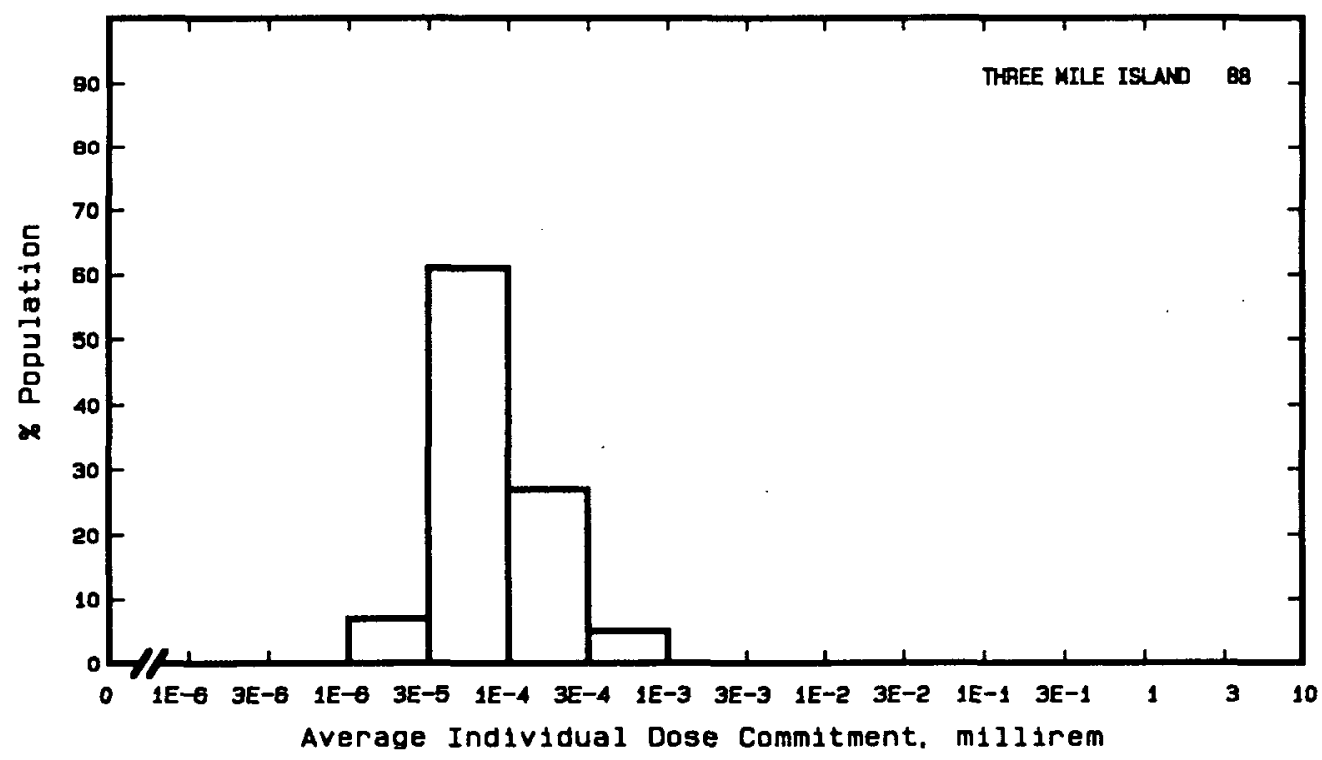

(a) Doses include releases from the TMI 2 / Epicore. 
Site: TROJAN

PRESCOTT, OREGON

Location: $\quad$ N $46.0408^{\circ} \quad$ W $122.8844^{\circ}$

POPULATION DATA

Total Population Within 2-to-80-km Region: 1.5E6

Major Metropolitan Centers Within Region:

Center

Portland-Vancouver SMSA

Longview

Astoria

Forest Grove

Centralia
Population

$1,500,000$

35,000

15,000

14,000

13,000
Location

$60 \mathrm{~km} \quad \mathrm{SSE}$

$12 \mathrm{~km} \quad \mathrm{NNW}$

$72 \mathrm{~km}$ WNW

$58 \mathrm{~km} \quad \mathrm{SSW}$

$75 \mathrm{~km} \mathrm{~N}$

\section{SITE-SPECIFIC DATA - AIRBORNE PATHWAYS}

Average Annual State Production

of Crops and Animal Products

In $80-\mathrm{km}$ Radius Circle

Regional Productivity Factor:

Animal Grazing Factor:
Veg: $6.4 \mathrm{E} 7 \mathrm{kilogram}$

Milk: 3.7E7 liter

Meat: $2.6 \mathrm{E} 7 \mathrm{kilogram}$

0.9

0.75

Meteorology Period of Record: 1 SEP 71 - 31 AUG 74 Recovery: 90\%

\section{SITE-SPECIFIC DATA - WATERBORNE PATHWAYS via COLUMBIA RIVER}

Average River Flow

at Site: $230,000 \mathrm{ft}^{3} / \mathrm{s}$

Drinking Water:

Exposed Population: $560(\mathrm{a})$

Dilution Factor: 1

Fish:

Edible Harvest: $1.0 \mathrm{E} 6 \mathrm{~kg} / \mathrm{yr}$

Dilution Factor: 1

(a) Population of Rainier divided by 4 , since residents only there for $25 \%$ of the year. 
POPULATION DOSE-COMMITMENT ESTIMATES AND AVERAGE INDIVIDUAL DOSE-COMMITMENT HISTOGRAM FOR

TROJAN

Dose Commitments (person-rem) from Waterborne Pathways

Total Body GI-LII Ihyroid Bone Liver

$\begin{array}{llllll}\text { Infant } & 7.9 \mathrm{E}-07 & 8.0 \mathrm{E}-07 & 7.9 \mathrm{E}-07 & 9.6 \mathrm{E}-08 & 7.8 \mathrm{E}-07 \\ \text { Child } & 9.5 \mathrm{E}-05 & 8.1 \mathrm{E}-04 & 3.2 \mathrm{E}-05 & 3.0 \mathrm{E}-04 & 2.6 \mathrm{E}-04 \\ \text { Teen } & 1.1 \mathrm{E}-04 & 1.6 \mathrm{E}-03 & 2.4 \mathrm{E}-05 & 1.9 \mathrm{E}-04 & 2.2 \mathrm{E}-04 \\ \text { Adult } & 1.1 \mathrm{E}-03 & 1.4 \mathrm{E}-02 & 1.9 \mathrm{E}-04 & 1.2 \mathrm{E}-03 & 1.3 \mathrm{E}-03 \\ \text { TOTAL } & 1.3 \mathrm{E}-03 & 1.7 \mathrm{E}-02 & 2.5 \mathrm{E}-04 & 1.7 \mathrm{E}-03 & 1.8 \mathrm{E}-03\end{array}$

Dose Commitments (person-rem) from Airborne Pathways

Total Body GI-LII Thyroid Bone Liver Lung

Infant $\quad 4.5 \mathrm{E}-04 \quad 4.5 \mathrm{E}-04 \quad 1.1 \mathrm{E}-03 \quad 2.1 \mathrm{E}-04 \quad 4.5 \mathrm{E}-04 \quad 4.6 \mathrm{E}-04$

Child 7.5E-03 7.5E-03 $1.4 \mathrm{E}-02 \quad 2.4 \mathrm{E}-03 \quad 7.5 \mathrm{E}-03 \quad 7.8 \mathrm{E}-03$

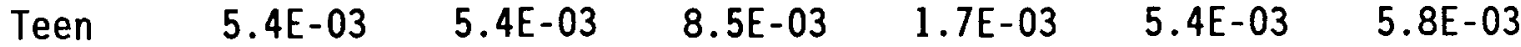

Adult $\quad 3.2 \mathrm{E}-02 \quad 3.2 \mathrm{E}-02 \quad 4.5 \mathrm{E}-02 \quad 1.0 \mathrm{E}-02 \quad 3.2 \mathrm{E}-02 \quad 3.3 \mathrm{E}-02$

TOTAL $4.5 \mathrm{E}-02 \quad 4.5 \mathrm{E}-02 \quad 6.8 \mathrm{E}-02 \quad 1.5 \mathrm{E}-02 \quad 4.5 \mathrm{E}-02 \quad 4.7 \mathrm{E}-02$

Production/Consumption factors:

Produce: 0.20

Milk: 0.17

Meat: 0.20

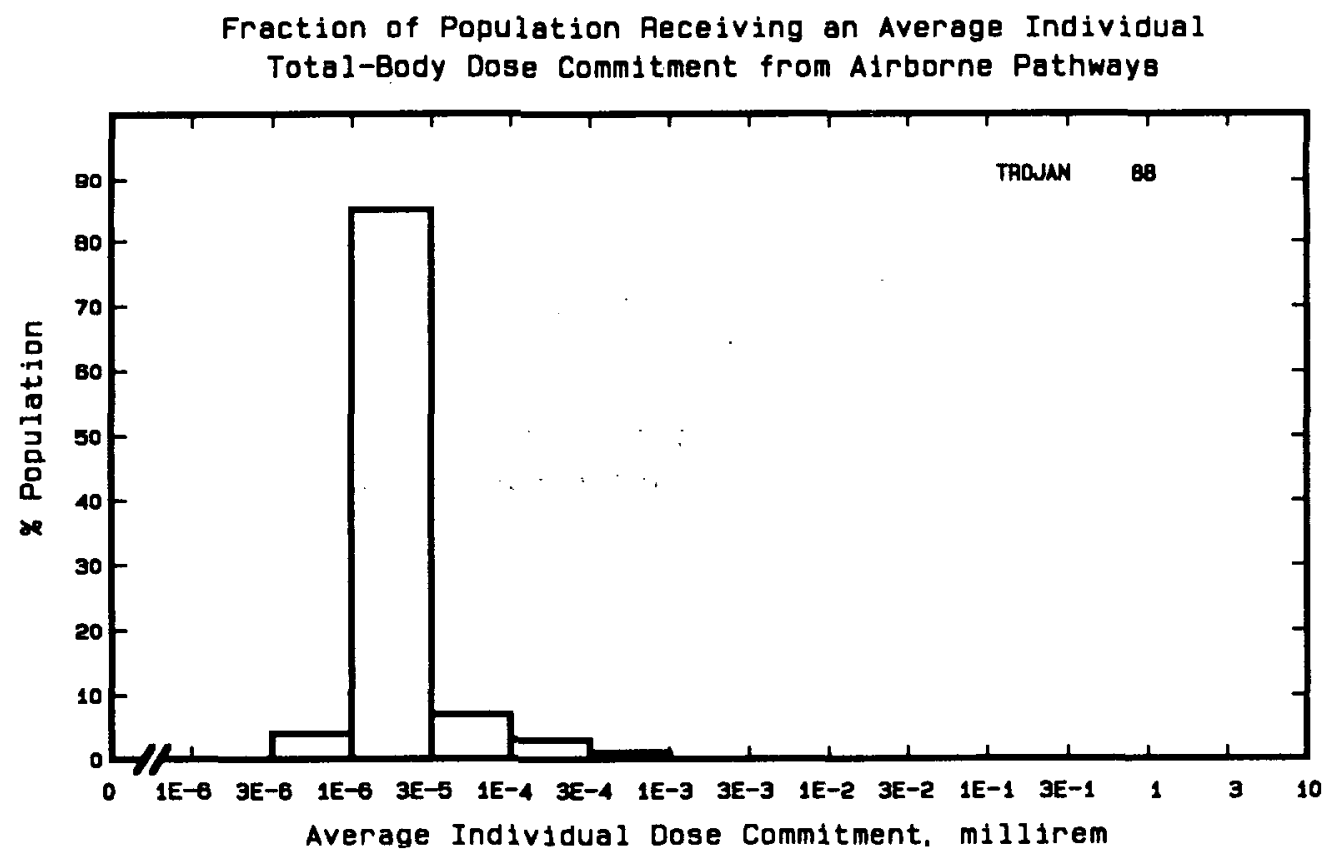


Site: TURKEY POINT

DADE COUNTY, FLORIDA

Location: N $25.4350^{\circ} \quad$ W $80.3314^{\circ}$

POPULATION DATA

Total Population Within 2-to-80-km Region: 2.9E6

Major Metropolitan Centers Within Region:

Center

Miami-Hialeah SMSA

Fort Lauderdale-Hollywood SMSA

Homestead

Upper Keys Division
Population Location

$2,000,000 \quad 41 \mathrm{~km} \quad \mathrm{NNE}$

$1,300,000 \quad 79 \mathrm{~km} \quad \mathrm{NNE}$

$24,000 \quad 16 \mathrm{~km} \quad W$

$18,000 \quad 42 \mathrm{~km} \quad \mathrm{~S}$

\section{SITE-SPECIFIC DATA - AIRBORNE PATHWAYS}

Average Annual State Production

of Crops and Animal Products

In $80-\mathrm{km}$ Radius Circle

Regional Productivity Factor:

Animal Grazing Factor:

Meteorology Period of Record: I JAN 73 - 31 DEC 73 Recovery: 98\%

SITE-SPECIFIC DATA - WATERBORNE PATHWAYS via BISCAYNE BAY

Average Dilution Flow

from Plants: $660 \mathrm{ft}^{3} / \mathrm{s}$

Fish:

Edible Harvest: (a)

Dilution Factor: 0.001

Invertebrates:

Edible Harvest: (a)

Dilution Factor: 0.002

(a) No catch data given in DES (1972), so generic consumption rates used (Table A-1). 
POPULATION DOSE-COMMITMENT ESTIMATES AND

AVERAGE INDIVIDUAL DOSE-COMMITMENT HISTOGRAM FOR

TURKEY POINT 3 AND 4

Dose Commitments (person-rem) from Waterborne Pathways

Total Body GI-LII $\underline{\text { Thyroid }}$ Bone $\underline{\text { Liver }}$

$\begin{array}{llllll}\text { Infant } & 0.0 \mathrm{E}+00 & 0.0 \mathrm{E}+00 & 0.0 \mathrm{E}+00 & 0.0 \mathrm{E}+00 & 0.0 \mathrm{E}+00 \\ \text { Child } & 7.7 \mathrm{E}-03 & 1.2 \mathrm{E}-02 & 7.7 \mathrm{E}-04 & 1.8 \mathrm{E}-02 & 1.2 \mathrm{E}-02 \\ \text { Teen } & 5.2 \mathrm{E}-03 & 2.4 \mathrm{E}-02 & 5.9 \mathrm{E}-04 & 1.0 \mathrm{E}-02 & 9.6 \mathrm{E}-03 \\ \text { Adult } & 3.2 \mathrm{E}-02 & 2.1 \mathrm{E}-01 & 4.2 \mathrm{E}-03 & 5.9 \mathrm{E}-02 & 5.6 \mathrm{E}-02 \\ \text { TOTAL } & 4.5 \mathrm{E}-02 & 2.5 \mathrm{E}-01 & 5.5 \mathrm{E}-03 & 8.6 \mathrm{E}-02 & 7.8 \mathrm{E}-02\end{array}$

Dose Commitments (person-rem) from Airborne Pathways

Total Body GI-LII Thyroid Bone Liver Lung

$\begin{array}{lllllll}\text { Infant } & 1.5 \mathrm{E}-03 & 1.5 \mathrm{E}-03 & 5.8 \mathrm{E}-03 & 8.0 \mathrm{E}-04 & 1.5 \mathrm{E}-03 & 1.6 \mathrm{E}-03 \\ \text { Child } & 2.2 \mathrm{E}-02 & 2.2 \mathrm{E}-02 & 4.6 \mathrm{E}-02 & 8.8 \mathrm{E}-03 & 2.2 \mathrm{E}-02 & 2.2 \mathrm{E}-02 \\ \text { Teen } & 1.6 \mathrm{E}-02 & 1.6 \mathrm{E}-02 & 2.6 \mathrm{E}-02 & 6.4 \mathrm{E}-03 & 1.6 \mathrm{E}-02 & 1.7 \mathrm{E}-02 \\ \text { Adult } & 9.7 \mathrm{E}-02 & 9.7 \mathrm{E}-02 & 1.3 \mathrm{E}-01 & 3.9 \mathrm{E}-02 & 9.8 \mathrm{E}-02 & 1.0 \mathrm{E}-01 \\ \text { TOTAL } & 1.4 \mathrm{E}-01 & 1.4 \mathrm{E}-01 & 2.1 \mathrm{E}-01 & 5.5 \mathrm{E}-02 & 1.4 \mathrm{E}-01 & 1.4 \mathrm{E}-01\end{array}$

Production/Consumption factors:

Produce: $0.020 \quad$ Milk: $0.12 \quad$ Meat: 0.12

Fraction of Population Receiving an Average Individual

Total-Body Dose Commitment from Alroorne Pathways

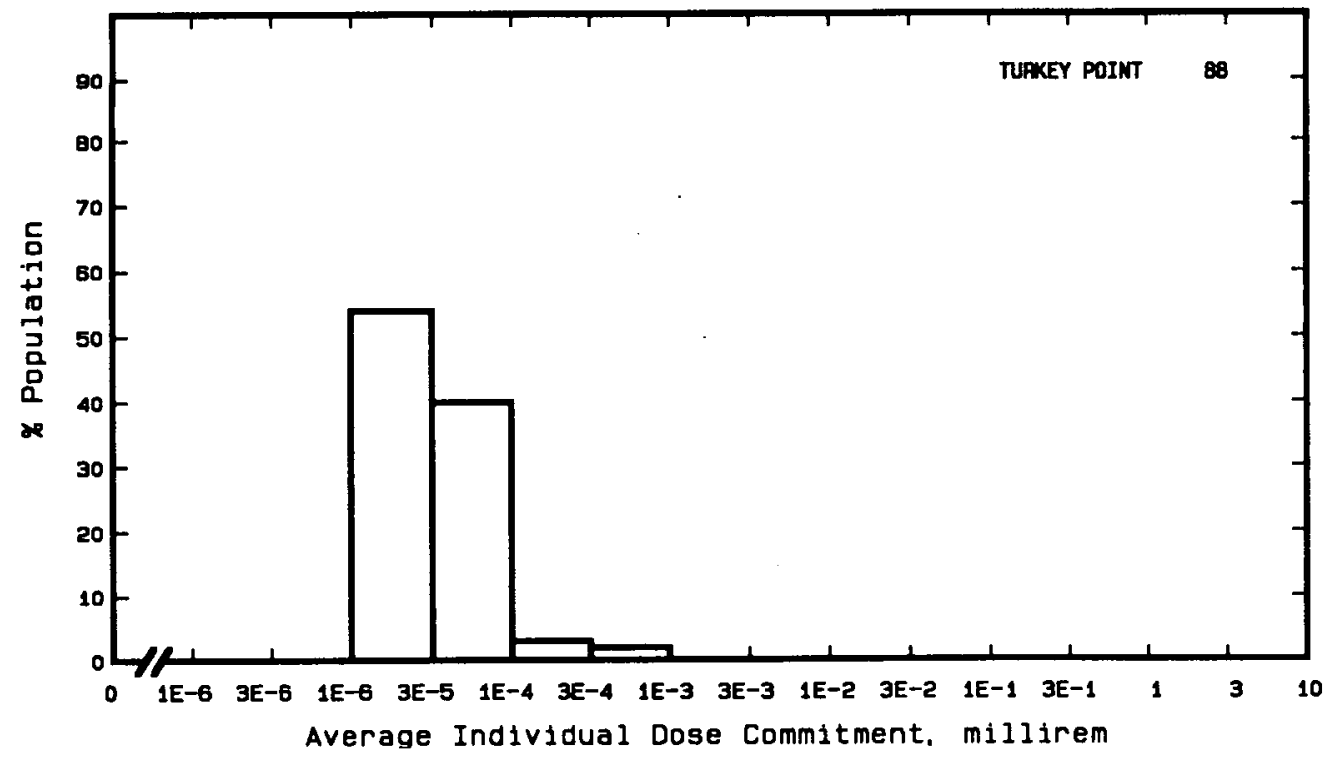


Site: VERMONT YANKEE

VERNON, VERMONT

Location: $\quad$ N $42.7803^{\circ} \quad$ W $72.5158^{\circ}$

POPULATION DATA

Total Population Within 2-to-80-km Region: 1.4E6

Major Metropolitan Centers Within Region:

Center Population Location

Springfield-Chicopee-Holyoke SMSA $570,000 \quad 70 \mathrm{~km} \quad \mathrm{~S}$

Worcester SMSA (1/2) $200,000 \quad 80 \mathrm{~km} \quad$ SE

Pittsfield $\quad 100,000 \quad 71 \mathrm{~km} \quad S W$

Fitchburg $\quad 44,000 \quad 63 \mathrm{~km}$ ESE

Leominster $\quad 39,000 \quad 68 \mathrm{~km}$ ESE

Keene $22,000 \quad 26 \mathrm{~km} \quad \mathrm{NW}$

SITE-SPECIFIC DATA - AIRBORNE PATHWAYS

Average Annual State Production

Veg: 4.4E6 kilogram

of Crops and Animal Products

In $80-\mathrm{km}$ Radius Circle

Milk: $7.3 \mathrm{E} 8$ liter

Meat: $2.7 \mathrm{E} 7$ kilogram

Regional Productivity Factor:

Animal Grazing Factor:

1

0.4

Meteorology Period of Record: 1 APR 75 - 31 MAR 76 Recovery: 97\%

SITE-SPECIFIC DATA - WATERBORNE PATHWAYS via CONNECTICUT RIVER at VERNON

POND

Average River Flow

at Site: $10,000 \mathrm{ft}^{3} / \mathrm{s}$

Drinking Water: None

Fish:

None 


\section{POPULATION DOSE-COMMITMENT ESTIMATES AND \\ AVERAGE INDIVIDUAL DOSE-COMMITMENT HISTOGRAM FOR \\ VERMONT YANKEE \\ Dose Commitments (person-rem) from Waterborne Pathways \\ Total Body GI-LII Thyroid Bone Liver}

Infant

Child

Teen

Adult

(No Waterborne Pathway Doses) (a)

TOTAL

Dose Commitments (person-rem) from Airborne Pathways

Total Body GI-LII Thyroid Bone Liver Lung

$\begin{array}{lllllll}\text { Infant } & 9.3 \mathrm{E}-04 & 9.2 \mathrm{E}-04 & 3.2 \mathrm{E}-03 & 5.9 \mathrm{E}-05 & 9.5 \mathrm{E}-04 & 1.0 \mathrm{E}-03 \\ \text { Child } & 1.1 \mathrm{E}-02 & 1.1 \mathrm{E}-02 & 2.6 \mathrm{E}-02 & 6.1 \mathrm{E}-04 & 1.1 \mathrm{E}-04 & 1.3 \mathrm{E}-02 \\ \text { Teen } & 7.4 \mathrm{E}-03 & 7.4 \mathrm{E}-03 & 1.5 \mathrm{E}-02 & 3.6 \mathrm{E}-04 & 7.4 \mathrm{E}-03 & 9.2 \mathrm{E}-03 \\ \text { Adult } & 3.9 \mathrm{E}-02 & 4.0 \mathrm{E}-02 & 6.9 \mathrm{E}-02 & 1.9 \mathrm{E}-03 & 4.0 \mathrm{E}-02 & 4.7 \mathrm{E}-02 \\ \text { TOTAL } & 5.9 \mathrm{E}-02 & 5.9 \mathrm{E}-02 & 1.1 \mathrm{E}-01 & 2.9 \mathrm{E}-03 & 5.9 \mathrm{E}-02 & 7.1 \mathrm{E}-02\end{array}$

Production/Consumption factors:

Produce: $0.015 \quad$ Milk: $3.8 \quad$ Meat: 0.23

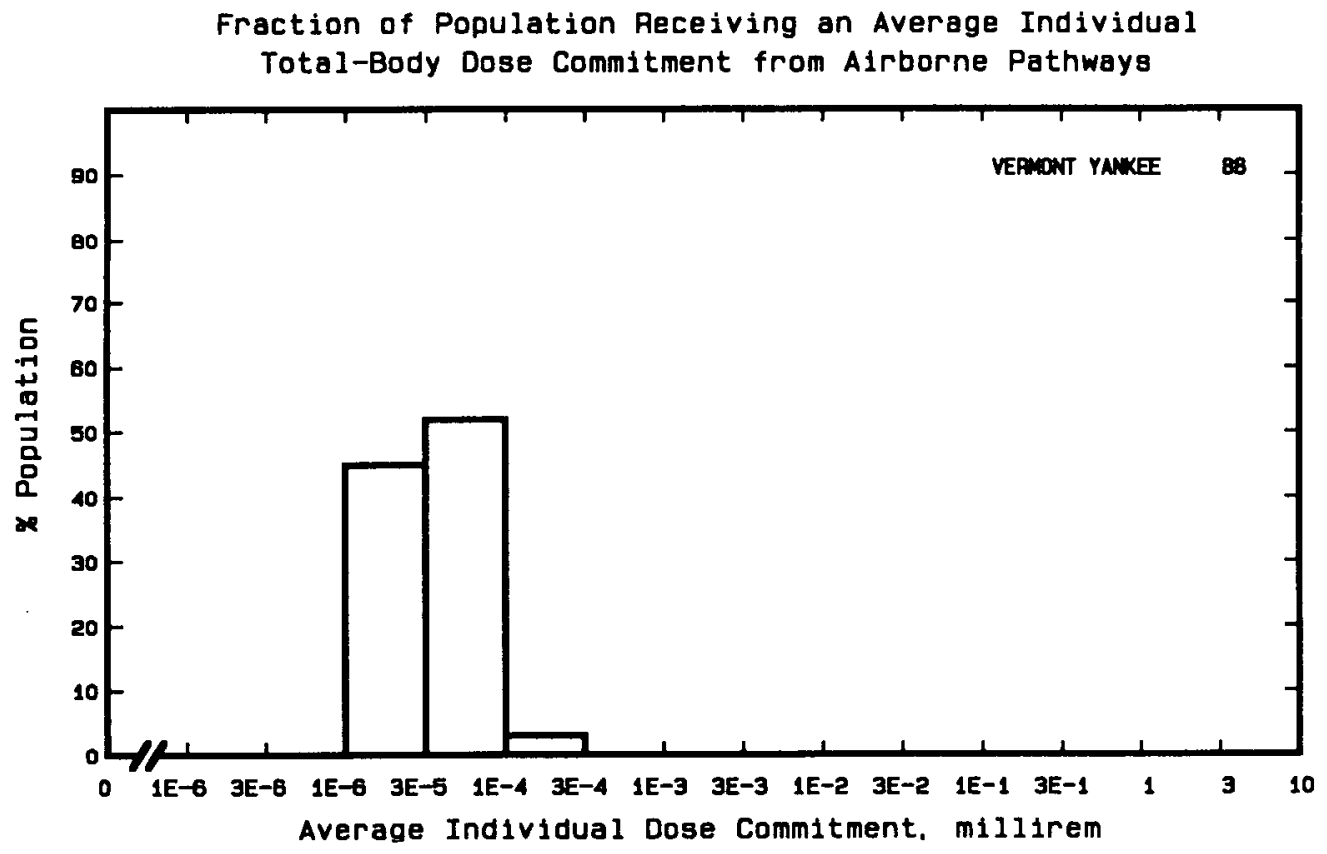

(a) No liquid releases reported (Tichler, et a 1. 1991). 
Site: VOGTLE

WAYNESBORO, GEORGIA

Location: $\quad$ N $33.1419^{\circ} \quad$ W $81.7647^{\circ}$

POPULATION DATA

Total Population Within 2-to-80-km Region: 6.2 E5

Major Metropolitan Centers Within Region:

\section{Center}

Augusta SMSA

Fort Gordon

Aiken

Statesboro
Population

400,000

18,000

17,000

17,000
Location

$40 \mathrm{~km} \quad \mathrm{NNW}$

$48 \mathrm{~km} \quad \mathrm{NW}$

$45 \mathrm{~km} \mathrm{~N}$

$76 \mathrm{~km} \mathrm{~S}$

SITE-SPECIFIC DATA - AIRBORNE PATHWAYS

Average Annual State Production

of Crops and Animal Products

In $80-\mathrm{km}$ Radius Circle

Regional Productivity Factor:

Animal Grazing Factor:
Veg: 8.8E6 kilogram

Milk: 7.0E7 liter

Meat: 8.1E7 kilogram

0.8

0.75

Meteorology Period of Record: 4 APR 77 - 31 MAR 81 Recovery: 93\%

Average River Flow at Site: $10,000 \mathrm{ft}^{3} / \mathrm{s}$

Drinking Water:

None

Fish:

Edible Harvest: $0.18 \mathrm{~kg} / \mathrm{yr}(\mathrm{a})$

Dilution Factor: 1

(a) Ten percent of population are assummed to obta in fish from river. Average individual consumption rate of $5 \mathrm{~g} / \mathrm{d}$ given in FES (1974) used in lieu of catch data. 


\section{POPULATION DOSE-COMMITMENT ESTIMATES AND \\ AVERAGE INDIVIDUAL DOSE-COMMITMENT HISTOGRAM FOR \\ VOGTLE 1}

Dose Commitments (person-rem) from Waterborne Pathways

\begin{tabular}{lccccc} 
& Total Body & GI-LLI & Thyroid & Bone & Liver \\
\cline { 2 - 3 } & $0.0 \mathrm{E}+00$ & $0.0 \mathrm{E}+00$ & $0.0 \mathrm{E}+00$ & $0.0 \mathrm{E}+00$ & $0.0 \mathrm{E}+00$ \\
Infant & $4.3 \mathrm{E}-04$ & $1.1 \mathrm{E}-02$ & $1.5 \mathrm{E}-04$ & $3.3 \mathrm{E}-04$ & $5.8 \mathrm{E}-04$ \\
Child & $3.2 \mathrm{E}-04$ & $2.2 \mathrm{E}-02$ & $1.2 \mathrm{E}-04$ & $1.9 \mathrm{E}-04$ & $5.1 \mathrm{E}-04$ \\
Teen & $2.1 \mathrm{E}-03$ & $1.9 \mathrm{E}-01$ & $8.5 \mathrm{E}-04$ & $1.2 \mathrm{E}-03$ & $3.2 \mathrm{E}-03$ \\
Adult & $2.9 \mathrm{E}-03$ & $2.2 \mathrm{E}-01$ & $1.1 \mathrm{E}-03$ & $1.7 \mathrm{E}-03$ & $4.3 \mathrm{E}-03$ \\
TOTAL & 2.03 & & & &
\end{tabular}

Dose Commitments (person-rem) from Airborne Pathways

Total Body GI-LLI Thyroid Bone Liver Lung

$\begin{array}{lllllll}\text { Infant } & 2.2 \mathrm{E}-04 & 2.2 \mathrm{E}-04 & 2.2 \mathrm{E}-04 & 3.1 \mathrm{E}-05 & 2.2 \mathrm{E}-04 & 2.2 \mathrm{E}-04 \\ \text { Child } & 3.2 \mathrm{E}-03 & 3.2 \mathrm{E}-03 & 3.2 \mathrm{E}-03 & 3.5 \mathrm{E}-04 & 3.2 \mathrm{E}-03 & 3.2 \mathrm{E}-03 \\ \text { Teen } & 2.1 \mathrm{E}-03 & 2.1 \mathrm{E}-03 & 2.1 \mathrm{E}-03 & 2.6 \mathrm{E}-04 & 2.1 \mathrm{E}-03 & 2.2 \mathrm{E}-03 \\ \text { Adult } & 1.2 \mathrm{E}-02 & 1.2 \mathrm{E}-02 & 1.2 \mathrm{E}-02 & 1.5 \mathrm{E}-03 & 1.2 \mathrm{E}-02 & 1.3 \mathrm{E}-02 \\ \text { TOTAL } & 1.8 \mathrm{E}-02 & 1.8 \mathrm{E}-02 & 1.8 \mathrm{E}-02 & 2.2 \mathrm{E}-03 & 1.8 \mathrm{E}-02 & 1.8 \mathrm{E}-02\end{array}$

Production/Consumption factors:

Produce: $0.058 \quad$ Milk: 0.69 Meat: 1.3

Fraction of Population Receiving an Average Individual

Total-Body Dose Commitment from Airborne Pathways

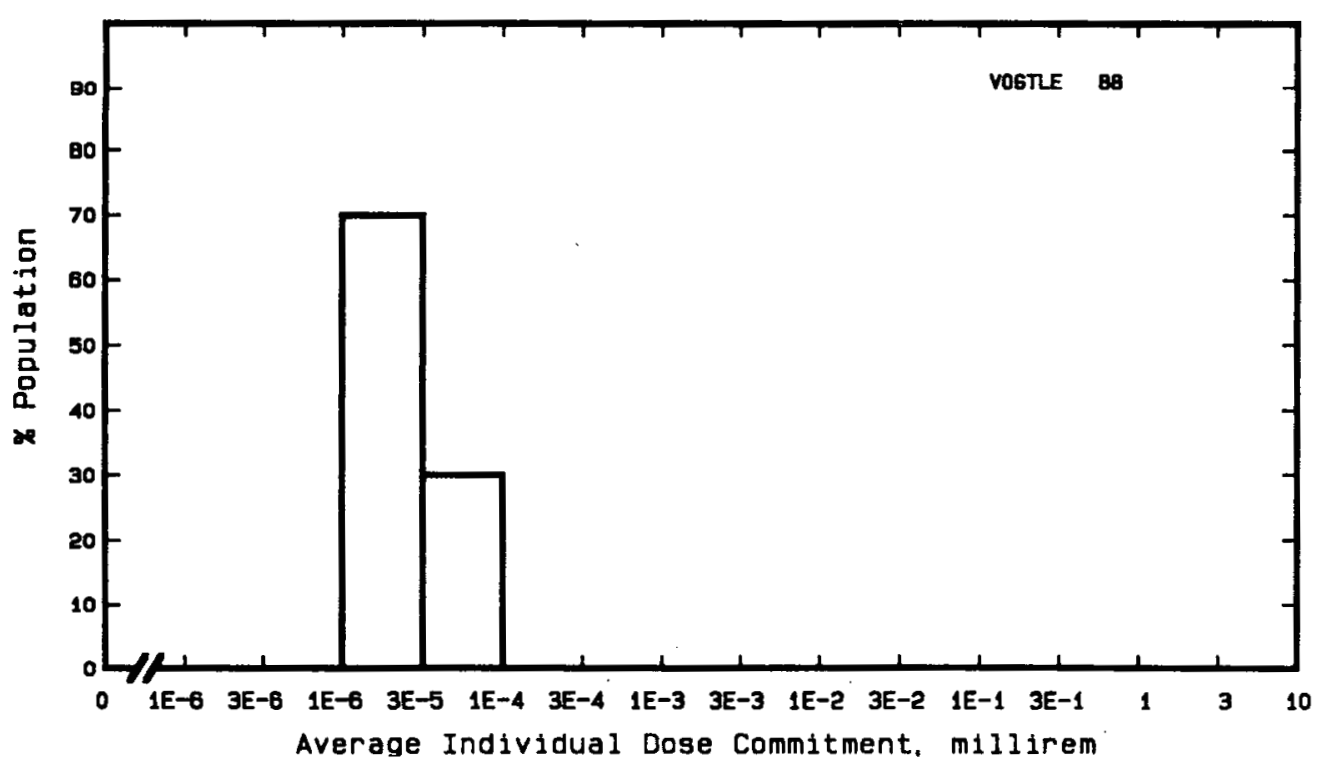


Site: WATERFORD

TAFT, LOUISIANA

Location: $\quad$ N $29.9953^{\circ} \quad$ W $90.4728^{\circ}$

POPULATION DATA

Total Population Within 2-to-80-km Region: $1.8 \mathrm{E} 6$

Major Metropolitan Centers Within Region:

Center

New Orleans MSA

Metaire

Kenner

Marrero

Houma
Population

$1,300,000$

140,000

30,000

29,000

31,000
Location

$32 \mathrm{~km} \quad \mathrm{E}$

$26 \mathrm{~km} \quad \mathrm{E}$

$16 \mathrm{~km} \quad \mathrm{E}$

$32 \mathrm{~km}$ ESE

$51 \mathrm{~km}$ SSW

SITE-SPECIFIC DATA - AIRBORNE PATHWAYS

Average Annual State Production

of Crops and Animal Products

In 80-km Radius Circle

Regional Productivity Factor:

Animal Grazing Factor:
Veg: $1.8 \mathrm{E} 6$ kilogram

Milk: 7.8E7 1iter

Meat: $6.1 \mathrm{E7}$ kilogram

0.6

1.0

Meteorology Period of Record: 2 FEB 77 - 1 FEB 78 Recovery: $89 \%$

SITE-SPECIFIC DATA - WATERBORNE PATHWAYS via MISSISSIPPI RIVER and GULF

Average River Flow

at Site: $490,000 \mathrm{ft}^{3} / \mathrm{s}$

Drinking Water:

Exposed Population: $1,100,000$

Dilution Factor: 1

Fish:

Edible Harvest: $4.5 \mathrm{E} 3 \mathrm{~kg} / \mathrm{yr}$ (River)

Dilution Factor: 1

Edible Harvest: $9.1 E 5 \mathrm{~kg} / \mathrm{yr}$ (Gulf)

Dilution Factor: 0.1

Gulf Invertebrates:

Edible Harvest: $5.1 \mathrm{E} 6 \mathrm{~kg} / \mathrm{yr}$

Dilution Factor: 0.1 


\section{POPULATION DOSE-COMMITMENT ESTIMATES AND \\ AVERAGE INDIVIDUAL DOSE-COMMITMENT HISTOGRAM FOR \\ WATERFORD 3}

Dose Commitments (person-rem) from Waterborne Pathways

Total Body GI-LLI Ihyroid Bone Liver

$\begin{array}{llllll}\text { Infant } & 1.0 \mathrm{E}-03 & 9.9 \mathrm{E}-04 & 8.4 \mathrm{E}-03 & 5.2 \mathrm{E}-04 & 1.6 \mathrm{E}-03 \\ \text { Child } & 1.2 \mathrm{E}-02 & 1.2 \mathrm{E}-02 & 6.2 \mathrm{E}-02 & 6.2 \mathrm{E}-03 & 1.6 \mathrm{E}-02 \\ \text { Teen } & 5.1 \mathrm{E}-03 & 6.2 \mathrm{E}-03 & 2.0 \mathrm{E}-02 & 1.8 \mathrm{E}-03 & 6.5 \mathrm{E}-03 \\ \text { Adult } & 4.5 \mathrm{E}-03 & 5.4 \mathrm{E}-02 & 1.5 \mathrm{E}-01 & 1.2 \mathrm{E}-02 & 5.0 \mathrm{E}-02 \\ \text { TOTAL } & 6.3 \mathrm{E}-02 & 7.3 \mathrm{E}-02 & 2.4 \mathrm{E}-01 & 2.0 \mathrm{E}-02 & 7.6 \mathrm{E}-02\end{array}$

Dose Commitments (person-rem) from Airborne Pathways

Total Body GI-LLI Ihyroid Bone Liver Lung

$\begin{array}{lllllll}\text { Infant } & 6.0 \mathrm{E}-03 & 6.0 \mathrm{E}-03 & 6.4 \mathrm{E}-03 & 4.3 \mathrm{E}-03 & 6.0 \mathrm{E}-03 & 6.4 \mathrm{E}-03 \\ \text { Child } & 7.6 \mathrm{E}-02 & 7.6 \mathrm{E}-02 & 7.8 \mathrm{E}-02 & 4.8 \mathrm{E}-02 & 7.6 \mathrm{E}-02 & 8.1 \mathrm{E}-02 \\ \text { Teen } & 5.6 \mathrm{E}-02 & 5.6 \mathrm{E}-02 & 5.8 \mathrm{E}-02 & 3.5 \mathrm{E}-02 & 5.6 \mathrm{E}-02 & 6.4 \mathrm{E}-02 \\ \text { Adult } & 3.4 \mathrm{E}-01 & 3.4 \mathrm{E}-01 & 3.4 \mathrm{E}-01 & 2.1 \mathrm{E}-01 & 3.4 \mathrm{E}-01 & 3.6 \mathrm{E}-01 \\ \text { TOTAL } & 4.8 \mathrm{E}-01 & 4.8 \mathrm{E}-01 & 4.9 \mathrm{E}-01 & 3.0 \mathrm{E}-01 & 4.8 \mathrm{E}-01 & 5.1 \mathrm{E}-01\end{array}$

Production/Consumption factors:

Produce: $0.0030 \quad$ Milk: 0.20 Meat: 0.25

Fraction of Population Receiving an Average Individual

Total-Body Dose Commitment from Airborne Pathways

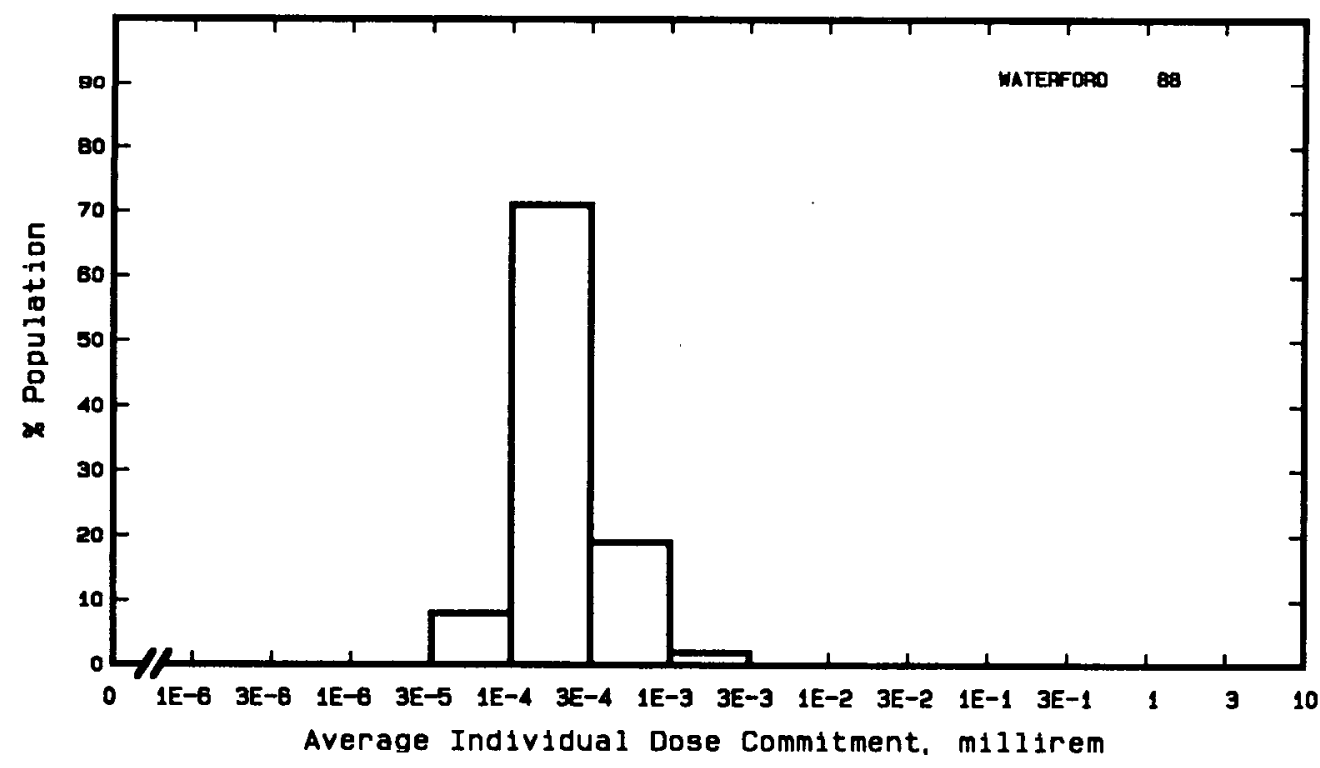


Site: WNP-2

RICHLAND, WASHINGTON

Location: $\quad$ N $46.2833^{\circ} \quad$ W $119.2916^{\circ}$

POPULATION DATA

Total Population Within 2-to-80-km Region: 2.8E5

Major Metropolitan Centers Within Region:

Center Population Location

Richland $\quad 36,000 \quad 14 \mathrm{~km} \quad \mathrm{~S}$

Kennewick $\quad 37,000 \quad 26 \mathrm{~km}$ SSE

$\begin{array}{llll}\text { Pasco } & 19,000 & 28 \mathrm{~km} & \text { SSE }\end{array}$

Moses Lake $\quad 11,000 \quad 73 \mathrm{~km} \mathrm{~N}$

SITE-SPECIFIC DATA - AIRBORNE PATHWAYS

Average Annual State Production

of Crops and Animal Products

In 80-km Radius Circle

Regional Productivity Factor:

Animal Grazing Factor:
Veg: 2.1E8 kilogram

Milk: 1.2E8 liter

Meat: $3.2 E 7$ kilogram

0.8

0.7

Meteorology Period of Record: 1 APR 75 - 31 MAR 76 Recovery: 93\%

SITE-SPECIFIC DATA - WATERBORNE PATHWAYS via COLUMBIA RIVER

Average River Flow

at Site: $115,000 \mathrm{ft}^{3} / \mathrm{s}$

Drinking Water:

Exposed Population: 75,000

Fish:

Edible Harvest: $7.5 \mathrm{E} 3 \mathrm{~kg} / \mathrm{yr}$

Dilution Factor: 1

(a) Effective factor for the cities of Richland, Kennewick, and Pasco accounting for only partial dilution. 


\section{POPULATION DOSE-COMMITMENT ESTIMATES AND \\ AVERAGE INDIVIDUAL DOSE-COMMITMENT HISTOGRAM FOR}

WNP-2

Dose Commitments (person-rem) from Waterborne Pathways

Total Body GI-LII Ihyroid Bone Liver

$\begin{array}{llllll}\text { Infant } & 3.4 \mathrm{E}-06 & 3.6 \mathrm{E}-06 & 1.9 \mathrm{E}-05 & 3.0 \mathrm{E}-06 & 6.1 \mathrm{E}-06 \\ \text { Child } & 4.2 \mathrm{E}-05 & 3.8 \mathrm{E}-05 & 1.4 \mathrm{E}-05 & 3.4 \mathrm{E}-05 & 6.4 \mathrm{E}-05 \\ \text { Teen } & 1.8 \mathrm{E}-05 & 1.9 \mathrm{E}-05 & 4.6 \mathrm{E}-05 & 9.7 \mathrm{E}-06 & 2.5 \mathrm{E}-05 \\ \text { Adult } & 1.6 \mathrm{E}-04 & 1.7 \mathrm{E}-04 & 3.4 \mathrm{E}-04 & 6.3 \mathrm{E}-05 & 1.9 \mathrm{E}-04 \\ \text { TOTAL } & 2.2 \mathrm{E}-04 & 2.3 \mathrm{E}-04 & 5.5 \mathrm{E}-04 & 1.1 \mathrm{E}-04 & 2.8 \mathrm{E}-04\end{array}$

Dose Commitments (person-rem) from Airborne Pathways

Total Body GI-LII Thyroid Bone Liver Lung

$\begin{array}{lllllll}\text { Infant } & 4.8 \mathrm{E}-04 & 5.7 \mathrm{E}-04 & 1.7 \mathrm{E}-02 & 4.4 \mathrm{E}-04 & 6.8 \mathrm{E}-04 & 4.0 \mathrm{E}-04 \\ \text { Child } & 5.8 \mathrm{E}-03 & 4.4 \mathrm{E}-03 & 1.5 \mathrm{E}-01 & 5.1 \mathrm{E}-03 & 7.2 \mathrm{E}-03 & 5.0 \mathrm{E}-03 \\ \text { Teen } & 3.5 \mathrm{E}-03 & 3.5 \mathrm{E}-03 & 6.3 \mathrm{E}-02 & 3.1 \mathrm{E}-03 & 4.4 \mathrm{E}-03 & 3.9 \mathrm{E}-03 \\ \text { Adult } & 1.9 \mathrm{E}-02 & 2.0 \mathrm{E}-02 & 2.4 \mathrm{E}-01 & 1.7 \mathrm{E}-02 & 2.2 \mathrm{E}-02 & 2.1 \mathrm{E}-02 \\ \text { TOTAL } & 2.9 \mathrm{E}-02 & 2.9 \mathrm{E}-02 & 4.7 \mathrm{E}-01 & 2.6 \mathrm{E}-02 & 3.4 \mathrm{E}-02 & 3.0 \mathrm{E}-02\end{array}$

Production/Consumption factors:

Produce: $3.1 \quad$ Milk: $2.7 \quad$ Meat: 1.1

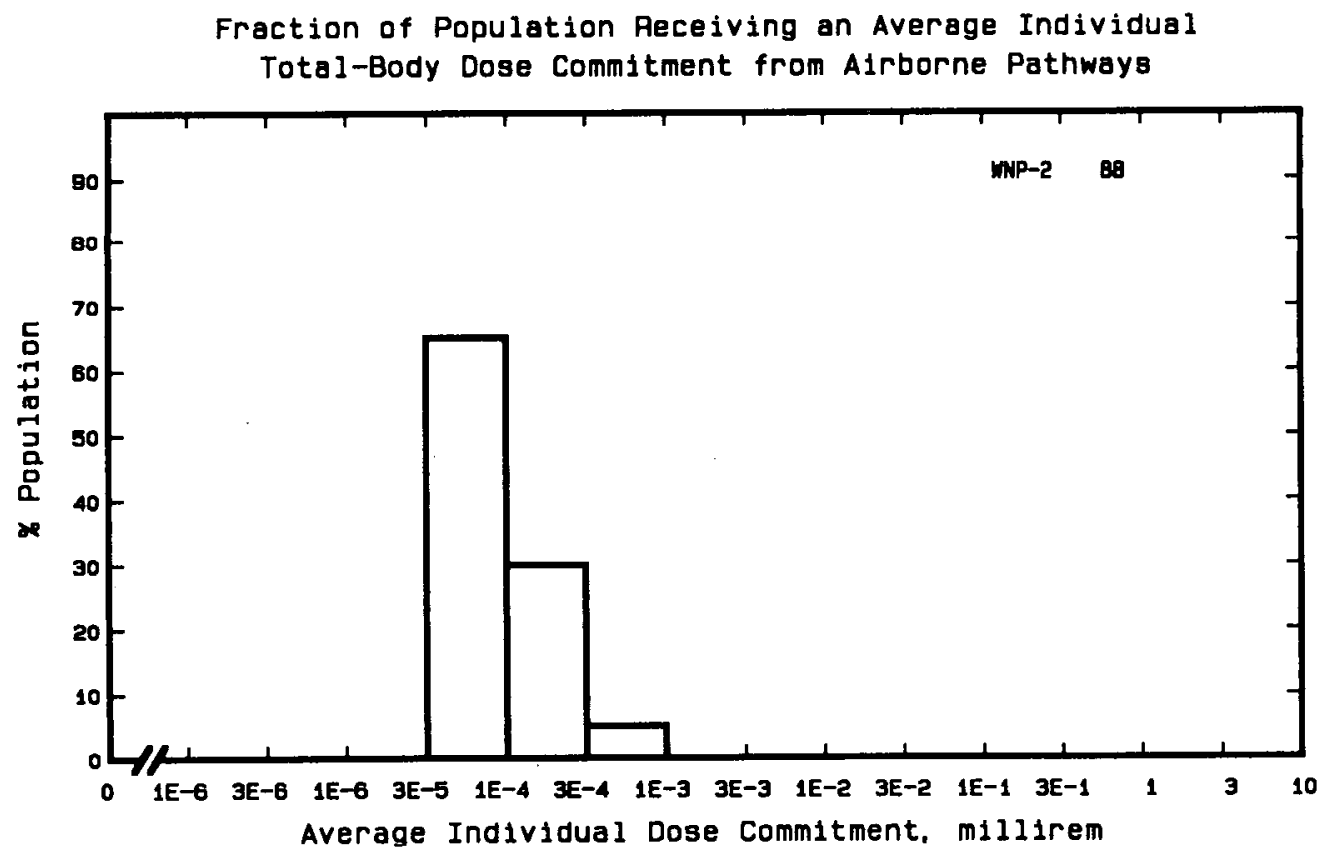


Site: WOLF CREEK

BURLINGTON, KANSAS

Location: $\quad \mathrm{N} 39.0267^{\circ} \quad$ W $84.7233^{\circ}$

POPULATION DATA

Total Population Within 2-to-80-km Region: 1.9E5

Major Metropolitan Centers Within Region:

Center

Chanute

Emporia

Ottawa
Population

11,000

26,000

11,000
Location

$62 \mathrm{~km} \quad$ SSE

$42 \mathrm{~km}$ WNW

$58 \mathrm{~km} \quad \mathrm{NW}$

SITE-SPECIFIC DATA - AIRBORNE PATHWAYS

Average Annual State Production

of Crops and Animal Products

In 80-km Radius Circle

Regional Productivity Factor:

Animal Grazing Factor:
Veg: 2.4E8 kilogram

Milk: $6.5 \mathrm{E} 7$ liter

Meat: $1.6 \mathrm{E} 8$ kilogram

0.95

0.5

Meteorology Period of Record: 3 years

Recovery: $93 \%$

Average River Flow

$$
\text { at Site: } 1,300 \mathrm{ft}^{3} / \mathrm{s}
$$

Drinking Water:

None

Fish:

Edible Harvest: $1.8^{(a)} \mathrm{kg} / \mathrm{yr}$ Dilution Factor: 1

(a) Average individual consumption rate of $5 \mathrm{~g} / \mathrm{d}$ given in FES (1982). 


\section{POPULATION DOSE-COMMITMENT ESTIMATES AND \\ AVERAGE INDIVIDUAL DOSE-COMMITMENT HISTOGRAM FOR \\ WOLF CREEK}

Dose Commitments (person-rem) from Waterborne Pathways

Total Body GI-LLI Thyroid Bone Liver

$\begin{array}{llllll}\text { Infant } & 0.0 \mathrm{E}+00 & 0.0 \mathrm{E}+00 & 0.0 \mathrm{E}+00 & 0.0 \mathrm{E}+00 & 0.0 \mathrm{E}+00 \\ \text { Child } & 1.0 \mathrm{E}-02 & 4.4 \mathrm{E}-02 & 2.4 \mathrm{E}-03 & 3.5 \mathrm{E}-02 & 4.1 \mathrm{E}-02 \\ \text { Teen } & 1.5 \mathrm{E}-02 & 9.1 \mathrm{E}-02 & 2.02 \mathrm{E}-03 & 2.1 \mathrm{E}-02 & 3.5 \mathrm{E}-02 \\ \text { Adult } & 1.5 \mathrm{E}-01 & 7.9 \mathrm{E}-01 & 1.4 \mathrm{E}-02 & 1.2 \mathrm{E}-01 & 2.1 \mathrm{E}-01 \\ \text { TOTAL } & 1.8 \mathrm{E}-01 & 9.2 \mathrm{E}-01 & 1.9 \mathrm{E}-02 & 1.8 \mathrm{E}-01 & 2.9 \mathrm{E}-01\end{array}$

Dose Commitments (person-rem) from Airborne Pathways

Total Body GI-LLI Thyroid Bone Liver Lung

$\begin{array}{lllllll}\text { Infant } & 1.1 \mathrm{E}-04 & 1.1 \mathrm{E}-04 & 1.1 \mathrm{E}-04 & 2.8 \mathrm{E}-05 & 1.1 \mathrm{E}-04 & 1.1 \mathrm{E}-04 \\ \text { Child } & 2.2 \mathrm{E}-03 & 2.2 \mathrm{E}-03 & 2.2 \mathrm{E}-03 & 3.2 \mathrm{E}-04 & 2.2 \mathrm{E}-03 & 2.2 \mathrm{E}-03 \\ \text { Teen } & 1.3 \mathrm{E}-03 & 1.3 \mathrm{E}-03 & 1.3 \mathrm{E}-03 & 2.3 \mathrm{E}-04 & 1.3 \mathrm{E}-03 & 1.3 \mathrm{E}-03 \\ \text { Aduit } & 7.0 \mathrm{E}-03 & 7.0 \mathrm{E}-03 & 7.0 \mathrm{E}-03 & 1.4 \mathrm{E}-03 & 7.0 \mathrm{E}-03 & 7.1 \mathrm{E}-03 \\ \text { TOTAL } & 1.1 \mathrm{E}-02 & 1.1 \mathrm{E}-02 & 1.1 \mathrm{E}-02 & 2.0 \mathrm{E}-03 & 1.1 \mathrm{E}-02 & 1.1 \mathrm{E}-02\end{array}$

Production/Consumption factors:

Produce: $6.3 \quad$ Milk: $2.5 \quad$ Meat: 10

Fraction of Population Receiving an Average Individual

Total-Body Dose Commitment from Alrborne Pathways

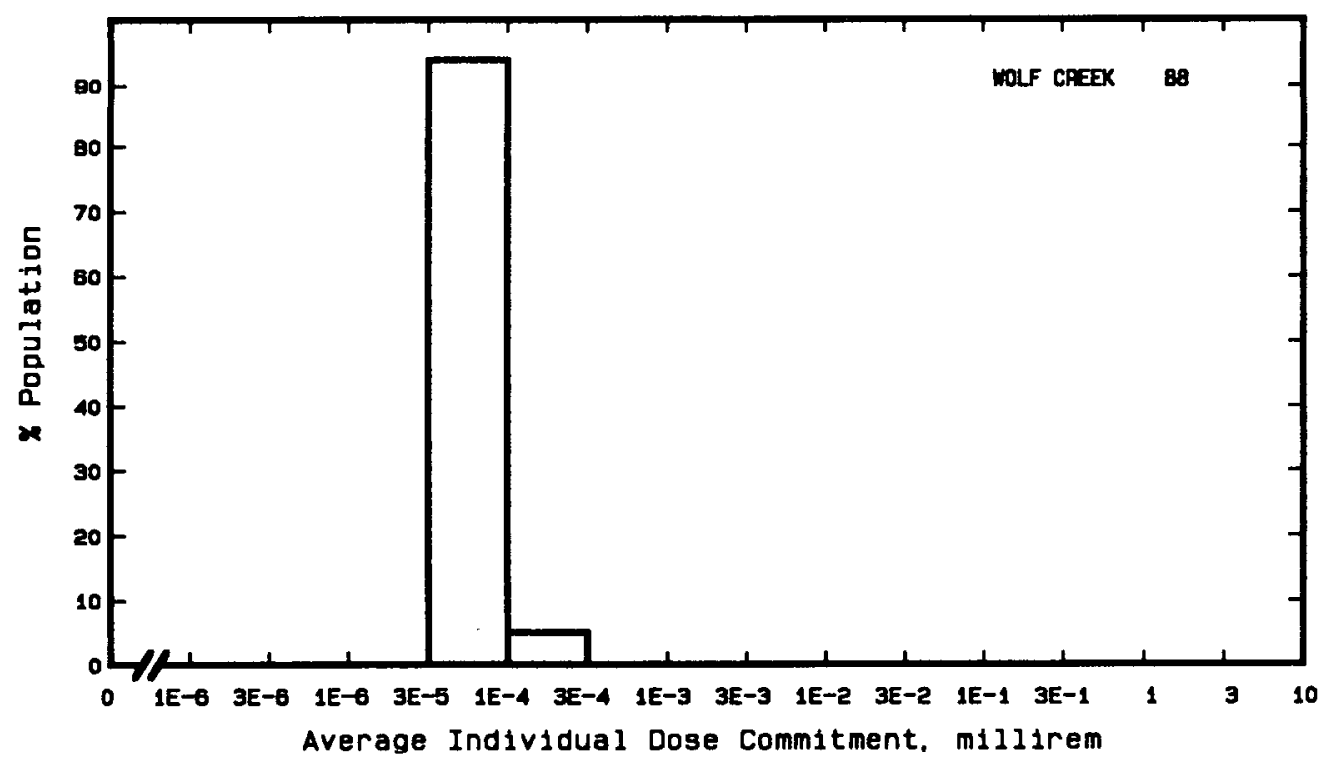


Site: YANKEE ROWE

Location: $\quad$ N $42.7281^{\circ}$
ROWE, MASSACHUSETTS

W $72.9289^{\circ}$

POPULATION DATA

Total Population Within 2-to-80-km Region: 1.6E6

Major Metropolitan Centers Within Region:

Center

Population

Location

Springfield-Chicopee-Holyoke SMSA

Albany

530,000

Pittsfield SMSA

100,000

92,000

Troy

57,000

Amherst

18,000

$74 \mathrm{~km} \quad$ SSE

$68 \mathrm{~km} \quad W$

$41 \mathrm{~km} \quad \mathrm{SW}$

$62 \mathrm{~km} \quad W$

$51 \mathrm{~km} \quad \mathrm{SE}$

\section{SITE-SPECIFIC DATA - AIRBORNE PATHWAYS}

Average Annual State Production

of Crops and Animal Products

In $80-\mathrm{km}$ Radius Circle

Regional Productivity Factor:

Animal Grazing Factor:
Veg: 2.0E7 kilogram

Milk: 2.6E8 liter

Meat: $1.6 \mathrm{E} 7 \mathrm{kilogram}$

1

0.5

Meteorology Period of Record: 1 OCT 71 - 30 SEP 72 Recovery: $94 \%$

SITE-SPECIFIC DATA - WATERBORNE PATHWAYS via DEERFIELD RIVER

Average River Flow

at Site: $570 \mathrm{ft}^{3} / \mathrm{s}$

Drinking Water:

None

Fish:

Edible Harvest: (a)

Dilution Factor: 0.025 (b)

(a) No catch data available, so generic consumption rates used (Table A-1).

(b) Ten percent of population obtain $25 \%$ of their fish from river. 
POPULATION DOSE-COMMITMENT ESTIMATES AND

AVERAGE INDIVIDUAL DOSE-COMMITMENT HISTOGRAM FOR

YANKEE ROWE

Dose Commitments (person-rem) from Waterborne Pathways

Total Body GI-LII $\underline{\text { Thyroid }}$ Bone Liver

$\begin{array}{llllll}\text { Infant } & 0.0 \mathrm{E}+00 & 0.0 \mathrm{E}+00 & 0.0 \mathrm{E}+00 & 0.0 \mathrm{E}+00 & 0.0 \mathrm{E}+00 \\ \text { Child } & 3.7 \mathrm{E}-02 & 2.3 \mathrm{E}-02 & 2.2 \mathrm{E}-02 & 1.7 \mathrm{E}-01 & 9.9 \mathrm{E}-02 \\ \text { Teen } & 4.1 \mathrm{E}-02 & 1.7 \mathrm{E}-02 & 1.3 \mathrm{E}-02 & 1.02 \mathrm{E}-01 & 7.9 \mathrm{E}-02 \\ \text { Adult } & 3.8 \mathrm{E}-01 & 1.1 \mathrm{E}-01 & 7.7 \mathrm{E}-02 & 5.9 \mathrm{E}-01 & 4.7 \mathrm{E}-01 \\ \text { TOTAL } & 4.5 \mathrm{E}-01 & 1.5 \mathrm{E}-01 & 1.1 \mathrm{E}-01 & 8.6 \mathrm{E}-01 & 6.5 \mathrm{E}-01\end{array}$

Dose Commitments (person-rem) from Airborne Pathways

Total Body GI-LII Thyroid Bone Liver Lung

$\begin{array}{lllllll}\text { Infant } & 1.8 \mathrm{E}-03 & 1.8 \mathrm{E}-03 & 1.9 \mathrm{E}-03 & 7.7 \mathrm{E}-03 & 1.8 \mathrm{E}-03 & 1.8 \mathrm{E}-03 \\ \text { Child } & 1.3 \mathrm{E}-02 & 1.3 \mathrm{E}-02 & 1.3 \mathrm{E}-02 & 5.4 \mathrm{E}-02 & 1.3 \mathrm{E}-02 & 1.3 \mathrm{E}-02 \\ \text { Teen } & 4.8 \mathrm{E}-03 & 4.8 \mathrm{E}-03 & 4.9 \mathrm{E}-03 & 1.7 \mathrm{E}-02 & 4.8 \mathrm{E}-03 & 5.0 \mathrm{E}-03 \\ \text { Adult } & 1.9 \mathrm{E}-02 & 1.9 \mathrm{E}-02 & 2.9 \mathrm{E}-02 & 5.3 \mathrm{E}-02 & 1.9 \mathrm{E}-02 & 1.9 \mathrm{E}-02 \\ \text { TOTAL } & 3.8 \mathrm{E}-02 & 3.8 \mathrm{E}-02 & 3.9 \mathrm{E}-02 & 1.3 \mathrm{E}-01 & 3.8 \mathrm{E}-02 & 3.9 \mathrm{E}-02\end{array}$

Production/Consumption factors:

Produce: 0.063

Milk: 1.2

Meat: 0.12

Fraction of Population Receiving an Average Individual

Total-Body Dose Commitment from Airborne Pathways

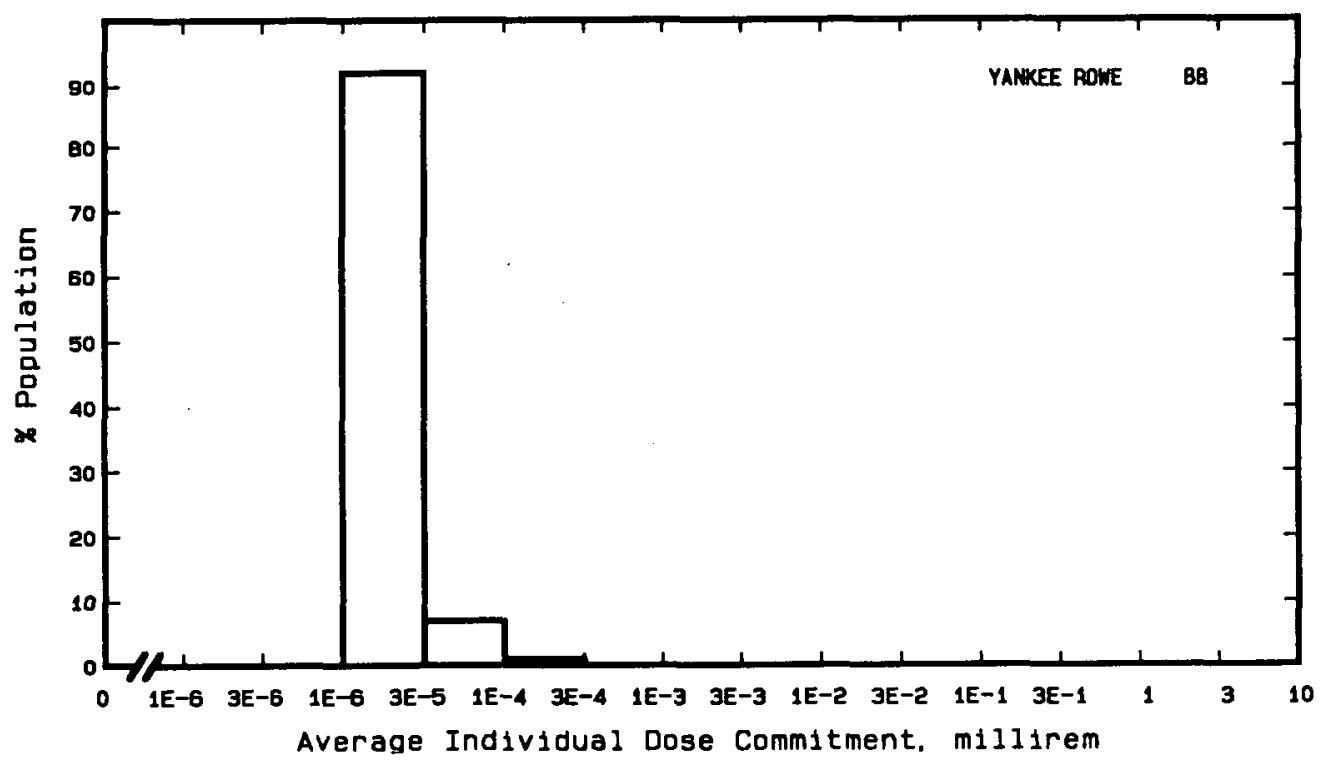


Site: ZION

ZION, ILLINOIS

Location: $\quad N \quad 42.4456^{\circ} \quad$ W $87.8022^{\circ}$

POPULATION DATA

Total Population Within 2-to-80-km Region: 7.3E6

Major Metropolitan Centers Within Region:

Center

Chicago SMSA (2/3)

Milwaukee SMSA

Racine SMSA

Kenosha SMSA

Waukesha
Population

$4,800,000$

$1,400,000$

170,000

120,000

51,000
Location

$66 \mathrm{~km} \mathrm{~S}$

$65 \mathrm{~km} \quad \mathrm{~N}$

$30 \mathrm{~km} \quad \mathrm{~N}$

$14 \mathrm{~km} \quad \mathrm{~N}$

$71 \mathrm{~km} \quad \mathrm{NNW}$

\section{SITE-SPECIFIC DATA - AIRBORNE PATHWAYS}

Average Annual State Production

of Crops and Animal Products

In $80-\mathrm{km}$ Radius Circle

Regional Productivity Factor:

Animal Grazing Factor:
Veg: $1.1 \mathrm{E8}$ kilogram

Mi1k: 1.8E8 1iter

Meat: $1.9 \mathrm{E} 8$ kilogram

0.5

0.5

Meteorology Period of Record: 1 JAN 74 - 31 DEC 75 Recovery: $88 \%$

Average Dilution Flow

from Plant: $2,700 \mathrm{ft}^{3} / \mathrm{s}$

Drinking Water:

Exposed Population: 6,900,000

Dilution Factor: 0.037

Edible Harvest: $5.0 \mathrm{E} 6 \mathrm{~kg} / \mathrm{yr}$

Dilution Factor: $0.01(\mathrm{~b})$

(a) Drinking water dilution factor est imated by averaging dilution factors derived from FES (1972) suitably weighted for population.

(b) Dilution factor derived from FES. 


\section{POPULATION DOSE-COMMITMENT ESTIMATES AND AVERAGE INDIVIDUAL DOSE-COMMITMENT HISTOGRAM FOR}

ZION 1 AND 2

Dose Commitments (person-rem) from Waterborne Pathways

Total Body GI-LII Ihyroid Bone Liver

$\begin{array}{llllll}\text { Infant } & 9.2 \mathrm{E}-02 & 9.2 \mathrm{E}-02 & 1.6 \mathrm{E}-01 & 4.4 \mathrm{E}-02 & 1.4 \mathrm{E}-01 \\ \text { Child } & 1.1 \mathrm{E}+00 & 1.2 \mathrm{E}+00 & 1.4 \mathrm{E}+00 & 7.2 \mathrm{E}-01 & 1.8 \mathrm{E}+00 \\ \text { Teen } & 5.5 \mathrm{E}-01 & 6.3 \mathrm{E}-01 & 5.1 \mathrm{E}-01 & 2.7 \mathrm{E}-01 & 8.0 \mathrm{E}-01 \\ \text { Adult } & 5.1 \mathrm{E}+00 & 5.6 \mathrm{E}+00 & 4.1 \mathrm{E}+00 & 1.6 \mathrm{E}+00 & 5.7 \mathrm{E}+00 \\ \text { TOTAL } & 6.8 \mathrm{E}+00 & 7.5 \mathrm{E}+00 & 6.2 \mathrm{E}+00 & 2.7 \mathrm{E}+00 & 8.4 \mathrm{E}+00\end{array}$

Dose Commitments (person-rem) from Airborne Pathways

Total Body GI-LII Thyroid Bone Liver Lung

$\begin{array}{lllllll}\text { Infant } & 4.3 \mathrm{E}-03 & 4.3 \mathrm{E}-03 & 4.8 \mathrm{E}-03 & 1.7 \mathrm{E}-03 & 4.3 \mathrm{E}-03 & 4.4 \mathrm{E}-03 \\ \text { Child } & 6.9 \mathrm{E}-02 & 6.9 \mathrm{E}-02 & 7.4 \mathrm{E}-02 & 1.8 \mathrm{E}-02 & 6.9 \mathrm{E}-02 & 7.1 \mathrm{E}-02 \\ \text { Teen } & 5.2 \mathrm{E}-02 & 5.2 \mathrm{E}-02 & 5.4 \mathrm{E}-02 & 1.3 \mathrm{E}-02 & 5.2 \mathrm{E}-02 & 5.5 \mathrm{E}-02 \\ \text { Adult } & 3.1 \mathrm{E}-01 & 3.1 \mathrm{E}-01 & 3.2 \mathrm{E}-01 & 8.1 \mathrm{E}-02 & 3.1 \mathrm{E}-01 & 3.2 \mathrm{E}-01 \\ \text { TOTAL } & 4.4 \mathrm{E}-01 & 4.4 \mathrm{E}-01 & 4.5 \mathrm{E}-01 & 1.1 \mathrm{E}-01 & 4.4 \mathrm{E}-01 & 4.5 \mathrm{E}-01\end{array}$

Production/Consumption factors:

Produce: $0.040 \quad$ Milk: 0.092 Meat: 0.16

Fraction of Population Receiving an Average Individual

Total-Body Dose Commitment from Airborne Pathways

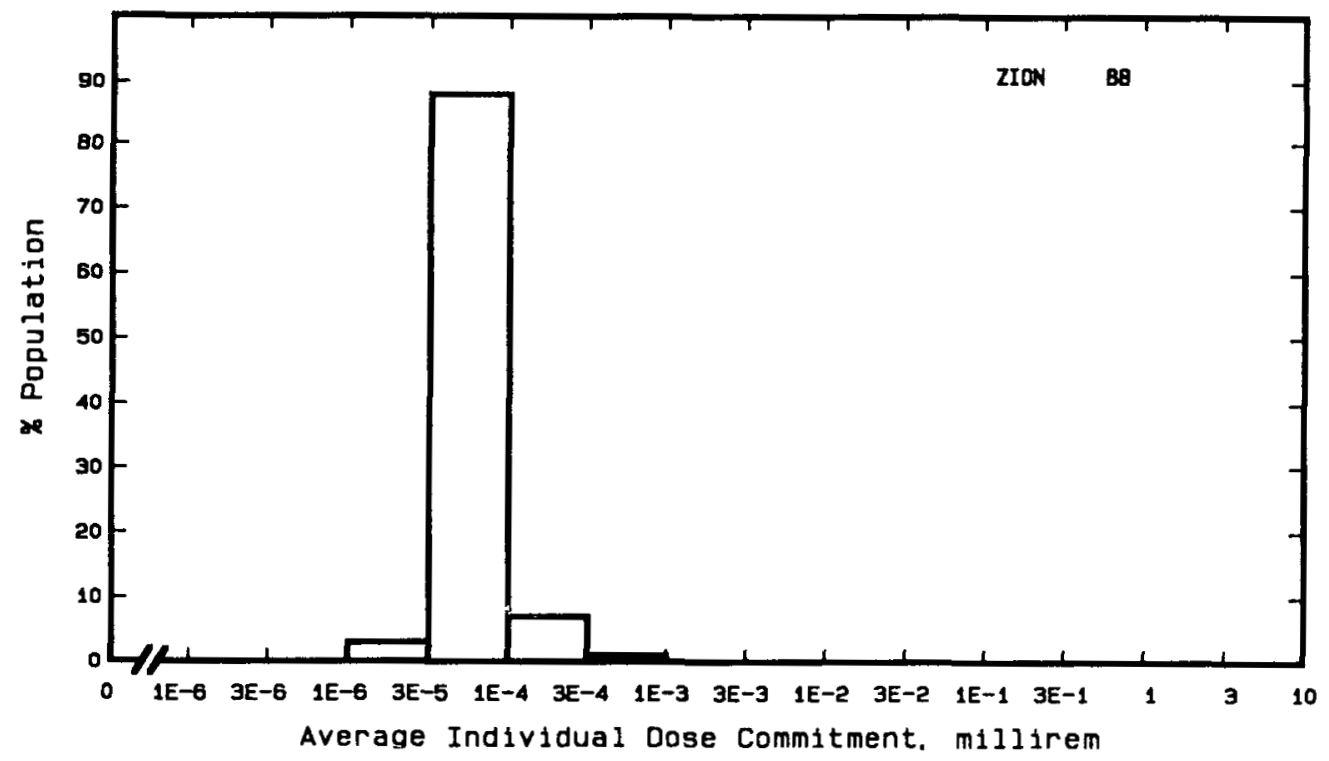




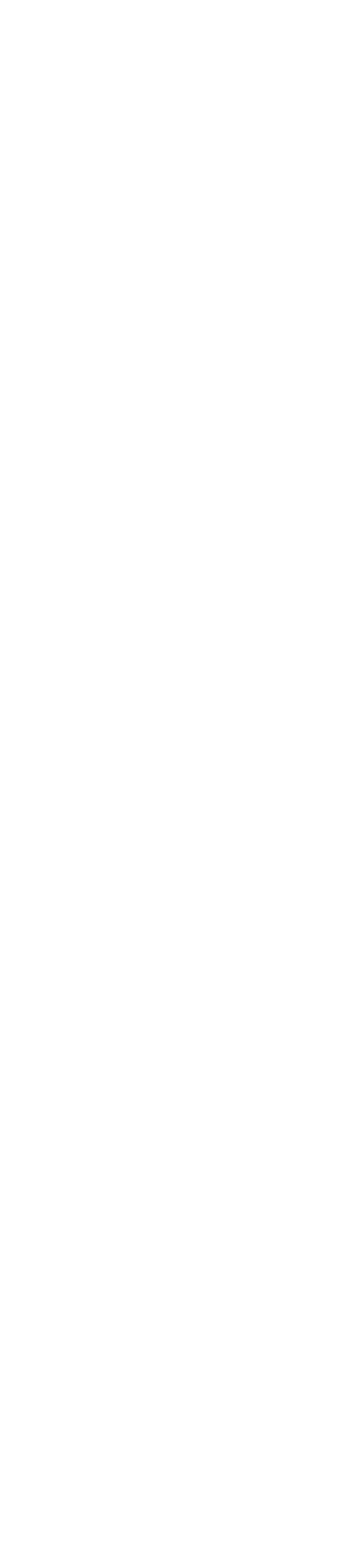




\section{REFERENCES}

Final Environmental Statement Concerning Proposed Rule-Making Action: Numerical Guides for Design Objectives and Limiting Conditions for Operation to Meet the Criterion "As Low As Practicable" for Radioactive Material in Light-Water-Cooled Nuclear Power Reactor Effluents. 1973. WASH-1258, Vol. 1, Directorate of Regulatory Standards, U.S. Atomic Energy Commission, Washington, D.C.

Tichler, J., K. Norden, and J. Congemi. 1991. Radioactive Materials Released from Nuclear Power Plants, Annual Report 1988. NUREG/CR-2907, BNL-NUREG-51581, Vo1. 9, U.S. Nuclear Regulatory Commission, Washington, D.C. 


\section{APPENDIX}

\section{MODELS AND GENERIC PARAMETERS}

The calculational models used were primarily those given in the Nuclear Regulatory Commission's Regulatory Guide 1.109, Rev. 1 (NRC 1977). Computer programs were written to use these models to generate population dose commitments for four age groups. The percentages of the population comprising the four age groups were $1.44 \%$, infant; $16.0 \%$, child; $11.7 \%$, teenager; and $70.9 \%$, adult (Population Estimates and Projections 1975). Where possible, the site- dependent parameters were taken from the environmental statements issued for each reactor (see Table 3). The generic parameters used for this study such as consumption rates, occupancy factors and holdup times are given in Tables A-1 and A-2 below. It should be noted that generic consumption rates for aquatic foods and inhalation rates are taken from Regulatory Guide 1.109 (NRC 1977). Bioaccumulation factors and terrestrial food transfer factors were taken from Regulatory Guide 1.109 (NRC 1977). Dose commitment factors for the four age groups were taken from Hoenes and Soldat (1977).

TABLE A-1. Generic Consumption Rates and Occupancy Factors Used for the Study of Average Members of the Population(a)

\begin{tabular}{|c|c|c|c|c|}
\hline Pathway & Infant & Child & Teen-ager & Adult \\
\hline $\begin{array}{l}\text { Fruits, vegetables and grain } \\
(\mathrm{kg} / \mathrm{yr})\end{array}$ & 0 & 200 & 240 & 190 \\
\hline Milk $(L / y r)$ & 170 & 170 & 200 & 110 \\
\hline Meat and poultry $(\mathrm{kg} / \mathrm{yr})$ & 0 & 37 & 59 & 95 \\
\hline Fish $(\mathrm{kg} / \mathrm{yr})^{(\mathrm{b})}$ & 0 & 2.2 & 5.2 & 6.9 \\
\hline Invertebrates $(\mathrm{kg} / \mathrm{yr})$ & 0 & 0.33 & 0.75 & 1.0 \\
\hline Drinking water $(\mathrm{L} / \mathrm{yr})$ & $170^{(c)}$ & 260 & 260 & 370 \\
\hline Inhalation $\left(\mathrm{m}^{3} / \mathrm{yr}\right)$ & 1400 (d) & 3700 & 8000 & 8000 \\
\hline $\begin{array}{l}\text { Air submersion and ground } \\
\text { irradiation occupancy } \\
\text { factor }\end{array}$ & 0.5 & 0.5 & 0.5 & 0.5 \\
\hline
\end{tabular}

(a) Regulatory Guide 1.109 (NRC 1977).

(b) Both fresh and salt water.

(c) Assumed to be equal to milk consumption.

(d) Same as for maximum individual. 
IABLE A-2. Holdup Times Between Harvest and Consumption of Foods (a)

\section{Food}

Fruits, grains and vegetables

$\operatorname{Milk}(b)$

Meat $(b)$

Aquatic foods (fish and invertebrates)

Drinking Water
Holdup Time (days)

14

4

20

7

1

(a) Regulatory Guide 1.109 (NRC 1977)

(b) Value given is time after milking or slaughter. For the portion of the time animals were fed stored feed, an additional 90 days was added to the holdup time

\section{SOURCE TERMS}

The doses were estimated using the measured releases as reported by the site operators for 1988 (Tichler, et al. 1991). (a) These releases include all radionuclides specified by the NRC to be measured and reported by the operators of all commercial nuclear power plants. Radionuclides given as a combination of parent-daughter isotopes such as $\mathrm{Y} / \mathrm{Sr}-90, \mathrm{Zr} / \mathrm{Nb}-95, \mathrm{Ba} / \mathrm{La}-140$, $\mathrm{I} / \mathrm{Xe}-133$ and $\mathrm{Pr} / \mathrm{Ce}-144$ were divided evenly between the parent and daughter.

The radionuclides used in this study, along with their decay constants, are given in Table A-3. Note that the "+D" after some of the nuclides indicates that the decay energy of the daughter is included with the parent. Thus, whenever a parent nuclide release is specified, the result of the dose calculation is as though an additional equilibrium amount of the daughter nuclide is specified. The daughter nuclide itself is included separately if it can be released independently of the parent and/or if it has a relatively long half-life.

\section{METEOROLOGY}

When more than one set of meteorological (joint frequency) data was available for a site, the one which appeared to be the most reliable was used to generate atmospheric transport factors. Factors were calculated for 16 compass points and for ten radii from 2 to $80 \mathrm{~km}$ (see Table A-4) using the NRC computer program XOQDOQ (Sagendorf and Goll 1977).

(a) Very shortlived isotopes such as $\mathrm{Kr}-90,91,93,94, \mathrm{Xe}-139,140,141,143$ and Rb-88M; those not likely to be produced; and those that were daughters whose decay energies were accounted for in the dose factor for the parent were not included in the dose. 
IABLE A-3. Radionuclides Considered in This Study

\begin{tabular}{|c|c|c|c|c|c|}
\hline No. & Nuclide & $\begin{array}{l}\text { Decay Constant } \\
(1 / \mathrm{sec})\end{array}$ & No. & Nuclide & $\begin{array}{l}\text { Decay Constant } \\
(1 / \mathrm{sec})\end{array}$ \\
\hline 1 & $H-3$ & $1.78 \mathrm{E}-09$ & 43 & $\mathrm{Nb}-97$ & $1.57 \mathrm{E}-04$ \\
\hline 2 & $\mathrm{Be}-10$ & $1.37 \mathrm{E}-14$ & 44 & Mo-99+D & $2.92 \mathrm{E}-06$ \\
\hline 3 & $C-14$ & $3.83 \mathrm{E}-12$ & 45 & Tc-99M & $3.19 \mathrm{E}-05$ \\
\hline 4 & $N-13$ & $1.16 \mathrm{E}-03$ & 46 & $R u-103+D$ & $2.02 \mathrm{E}-07$ \\
\hline 5 & $F-18$ & $1.05 \mathrm{E}-04$ & 47 & $R u-106+D$ & $2.17 E-08$ \\
\hline 6 & $\mathrm{Na}-22$ & $8.44 E-09$ & 48 & $A g-110 M+D$ & $3.19 E-08$ \\
\hline 7 & $\mathrm{Na}-24$ & $1.28 \mathrm{E}-05$ & 49 & $C d-115 M$ & $1.80 \mathrm{E}-07$ \\
\hline 8 & $A r-41$ & $1.05 \mathrm{E}-04$ & 50 & $C d-115$ & $3.60 \mathrm{E}-06$ \\
\hline 9 & Sc- -46 & $9.58 \mathrm{E}-08$ & 51 & $S n-125+D$ & $8.31 \mathrm{E}-07$ \\
\hline 10 & $\mathrm{Cr}-51$ & $2.89 \mathrm{E}-07$ & 52 & $S b-124$ & $1.33 \mathrm{E}-07$ \\
\hline 11 & $M n-54$ & $2.57 \mathrm{E}-08$ & 53 & Sb- $125+D$ & $8.06 E-09$ \\
\hline 12 & $M n-56$ & $7.47 \mathrm{E}-05$ & 54 & $\mathrm{Te}-132+\mathrm{D}$ & $2.47 \mathrm{E}-06$ \\
\hline 13 & $\mathrm{Fe}-55$ & $8.14 \mathrm{E}-09$ & 55 & $\mathrm{Te}-133 \mathrm{M}+\mathrm{D}$ & $2.09 \mathrm{E}-04$ \\
\hline 14 & $\mathrm{Fe}-59$ & $1.80 \mathrm{E}-07$ & 56 & $I-131+D$ & $9.97 \mathrm{E}-07$ \\
\hline 15 & Co-57 & $2.97 E-08$ & 57 & I -132 & $8.42 E-05$ \\
\hline 16 & Co-58 & $1.12 \mathrm{E}-07$ & 58 & I-133+D & $9.25 E-06$ \\
\hline 17 & Co-60 & 4.17E-09 & 59 & I - 134 & $2.20 \mathrm{E}-04$ \\
\hline 18 & $\mathrm{Ni}-57$ & $5.35 E-06$ & 60 & $I-135+D$ & $2.92 \mathrm{E}-05$ \\
\hline 19 & $\mathrm{Ni}-63$ & $2.20 \mathrm{E}-10$ & 61 & $\mathrm{Xe}-131 \mathrm{M}$ & $6.69 \mathrm{E}-07$ \\
\hline 20 & $\mathrm{Ni}-65$ & $7.64 \mathrm{E}-05$ & 62 & $X e-133 M$ & $3.61 \mathrm{E}-06$ \\
\hline 21 & $\mathrm{Cu}-64$ & $1.52 \mathrm{E}-05$ & 63 & $\mathrm{Xe}-133$ & $1.52 \mathrm{E}-06$ \\
\hline 22 & $2 n-65$ & $3.31 E-08$ & 64 & $X e-135 M$ & $7.56 \mathrm{E}-04$ \\
\hline 23 & $Z n-69 M+D$ & $1.39 E-05$ & 65 & $X e-135$ & $2.10 E-05$ \\
\hline 24 & As -76 & $7.32 \mathrm{E}-06$ & 66 & Xe-137 & $3.01 \mathrm{E}-03$ \\
\hline 25 & $\mathrm{Br}-82$ & $5.44 \mathrm{E}-06$ & 67 & $X e-138+D$ & $8.14 \mathrm{E}-04$ \\
\hline 26 & $\mathrm{Kr}-83 \mathrm{M}$ & $1.04 E-04$ & 68 & Cs -134 & $1.07 \mathrm{E}-08$ \\
\hline 27 & $\mathrm{Kr}-85 \mathrm{M}$ & $4.31 E-05$ & 69 & Cs -136 & $6.17 \mathrm{E}-07$ \\
\hline 28 & $\mathrm{Kr}-85$ & $2.05 E-09$ & 70 & $C s-137+D$ & $7.31 \mathrm{E}-10$ \\
\hline 29 & $\mathrm{Kr}-87$ & $1.52 E-04$ & 71 & Cs -138 & $3.58 \mathrm{E}-04$ \\
\hline 30 & $\mathrm{Kr}-88+\mathrm{D}$ & $6.89 \mathrm{E}-05$ & 72 & $C_{s}-139+D$ & $1.24 \mathrm{E}-03$ \\
\hline 31 & $\mathrm{Kr}-89$ & $3.64 E-03$ & 73 & $\mathrm{Ba}-139$ & $1.39 \mathrm{E}-04$ \\
\hline 32 & $\mathrm{Rb}-88$ & $6.53 \mathrm{E}-04$ & 74 & $\mathrm{Ba}-140+\mathrm{D}$ & $6.28 E-07$ \\
\hline 33 & $\mathrm{Rb}-89+\mathrm{D}$ & $7.61 \mathrm{E}-04$ & 75 & La-140 & $4.78 \mathrm{E}-06$ \\
\hline 34 & $\mathrm{Sr}-89+\mathrm{D}$ & $1.59 \mathrm{E}-07$ & 76 & La-141 & $4.97 \mathrm{E}-05$ \\
\hline 35 & $S r-90+D$ & $7.58 \mathrm{E}-10$ & 77 & $\mathrm{Ce}-141$ & $2.47 \mathrm{E}-07$ \\
\hline 36 & $S r-91+D$ & $2.03 E-05$ & 78 & $\mathrm{Ce}-144+\mathrm{D}$ & $2.83 E-08$ \\
\hline 37 & $S r-92+D$ & $7.11 \mathrm{E}-05$ & 79 & Eu-152 & $1.69 \mathrm{E}-09$ \\
\hline 38 & $Y-90$ & $3.01 \mathrm{E}-06$ & 80 & $E u-154$ & $2.55 \mathrm{E}-09$ \\
\hline 39 & $Y-91 M+D$ & $2.32 E-04$ & 81 & $W-187$ & $8.06 \mathrm{E}-06$ \\
\hline 40 & $Z r-95+D$ & $1.22 \mathrm{E}-07$ & 82 & Th-232+D & $1.57 E-18$ \\
\hline 41 & $Z r-97+D$ & $1.14 \mathrm{E}-05$ & 83 & $\mathrm{~Np}-239$ & $3.42 E-06$ \\
\hline 42 & $\mathrm{Nb}-95$ & $2.29 \mathrm{~F}-07$ & & & \\
\hline
\end{tabular}


IABLE A-4. Radius Intervals and Midpoints for Airborne Dose Calculations $(\mathrm{km})$

$\begin{array}{cc}\text { Interval } & \text { Midpoint } \\ 2-3 & \\ 3-4 & 2.5 \\ 4-6 & 3.5 \\ 6-9 & 5 \\ 9-14 & 7.5 \\ 14-20 & 11.5 \\ 20-30 & 17 \\ 30-40 & 25 \\ 40-60 & 35 \\ 60-80 & 50 \\ 0\end{array}$

The XOQDOQ program generates four sets of atmospheric transport factors:

- average annual atmospheric dilution factors, which are not corrected for cloud depletion or radioactive decay

- dilution factors that are only corrected for decay assuming a 2.26-day half-life

- dilution factors that are corrected for depletion and for decay assuming an 8-day half-1ife

- relative deposition per unit area.

These factors were used to estimate the dose from airborne releases using methods similar to the NRC GASPAR program (Eckerman et al. 1980). The transport factors used this year were the same as those used for the previous estimates. The assumptions used in the calculation of these transport factors were as follows:

- 50-m source height with no correction for plume rise or building wake effects

- semi-infinite cloud model with sector-average, Gaussian-plume dispersion no correction for terrain height variation.

Since information about height and locations at each site for the releases given in Tichler, et al. (1991) was unavailable, a single generic height of $50 \mathrm{~m}$ was used at each site for the release point. Because the heights and locations of releases are uncertain, estimates of dose to persons 
living within $2 \mathrm{~km}$ of the site could be in serious error; only persons living between 2 to $80 \mathrm{~km}$ from the site were included in the dose estimates.

\section{POPULATION}

The population distribution within 2 to $80 \mathrm{~km}$ around each site was determined from information supplied by the NRC from an updated reduction of 1980 census data (Sinisgalli 1982). Also the NRC supplied updated estimates of the number of people residing in major metropolitan centers within the $80-\mathrm{km}$ region around each site (Brauner 1982) and D. P. Cleary. (a) Population variations between 1980 and 1986 (population factors) were derived from census data (U. S. Bureau of the Census 1988).

\section{FOOD PRODUCTION VERSUS FOOD CONSUMPTION}

The total food production for the region within $80 \mathrm{~km}$ around each site was the product of the NRC state-wide productivity figure for each state and a site productivity factor. At some sites this total production may be more or less than the total consumption; i.e., population times average individual consumption (see Table A-1 for generic consumption rates). When production was more than consumption for a site, it was assumed that all persons in the 2-to-80-km region ate contaminated food; when production was less than consumption, it was assumed that dilution would occur because uncontaminated food would be shipped into the area from outside. Thus, the calculated doses for a particular food type were reduced in proportion to the ratio of pro-duction divided by consumption (production/consumption less than 1).

The dose to persons outside the $80-\mathrm{km} 1 \mathrm{imit}$ from food shipped out of the region, in the case of production being greater than consumption, is not included in this report because we are concerned only with the dose within the $80-\mathrm{km}$ radius. These production/consumption factors are given for reference as footnotes to the tables showing airborne dose commitment in the site Summaries section.

\section{DRINKING WATER}

The population between 2 and $80 \mathrm{~km}$ distance from each $\mathrm{plant}$ site exposed to drinking water contaminated with released radionuclides was generally obtained from the environmental statement (ES) for the plant. For all sites located on salt water, it was assumed that no dose was received from drinking water. The generic consumption rates used for drinking water are given in Table A-1.

(a) Letter from D. P. Cleary, U.S. Nuclear Regulatory Commission, to D. A. Baker, Pacific Northwest Laboratory, June 1987. 
The radionuclide concentration in the drinking water consumed by a population downstream from a site was usually estimated assuming $100 \%$ mixing of the plant effluent with the river. For lakes, an overall dilution factor was estimated from dilution factors given in the ES for each population center along the shore (within $80 \mathrm{~km}$ ) that consumes the contaminated lake water. These individual factors were weighted by population and averaged to obtain an effective dilution factor for the total population exposed to contaminated drinking water.

\section{AQUATIC FOOD}

Wherever possible, the fish-catch data from the plant ES were used to estimate aquatic food consumption rates for the population living within the region. When these data were not found in the ES or were considered unrealistic, the generic values of Table A-1 were used.

The average radionuclide concentration of the waters in which this food was harvested was estimated assuming an additional dilution over the effluent flow from the reactor. For rivers, it was assumed that the fish were caught in waters in which the plant effluent was completely diluted. For lakes, an additional factor as given in the ES was used; when none was given in the ES, a generic value of 0.01 was used. For ocean and bay sites, generic values of 0.001 and 0.002 were used for fish and invertebrates, respectively, if the ES yielded no values for these parameters. Invertebrates were not assumed to be caught in sufficient quantity at freshwater sites (river and lake) to affect the population dose and therefore were not included in the dose calculation. Any exceptions to these general guidelines are explained in the footnotes to the individual site summaries. 


\section{APPENDIX REFERENCES}

Baker, D. A., J. K. Soldat and E. C. Watson. 1977. Population Dose Commitments Due to Radioactive Releases from Nuclear Power Plant Sites in 1975. PNL-2439, Pacific Northwest Laboratory, Richland, Washington.

Brauner, A. 1982. Population Estimates, Nuclear Power Plant Nearby Population Concentrations. U.S. Nuclear Regulatory Commission, Washington, D.C.

Eckerman, K. F., F. J. Congel, A. K. Roecklein and W. J. Pasciak. 1980. Users Guide to GASPAR Code. NUREG-0597, U.S. Nuclear Regulatory Commission, Washington, D.C.

Hoenes, G. R., and J. K. Soldat. 1977. Age-Specific Radiation Dose Commitment Factors for a One-Year Chronic Intake. NUREG-0172, U.S. Nuclear Regulatory Commission, Washington, D.C.

Population Estimates and Projections, Current Population Reports. 1975. Series P-25, No. 541. U.S. Department of Commerce, Social and Economic Statistics Administration, Bureau of the Census.

Sagendorf, J. F., J. T. Goll, and W. F. Sandusky. 1982. X0QD0Q: Computer Program for the Meteorological Evaluation of Routine Effluent Releases at Nuclear Power Stations. NUREG/CR-2919, PNL-4380, U.S. Nuclear Regulatory Commission, Washington, D.C.

Sinisgalli, A. 1982. 1980 Residential Population Estimates, 0-80 Kilometers for Nuclear Power Plants. U. S. Nuclear Regulatory Commission, Washington, D.C.

Tichler, J., K. Norden, and J. Congemi. 1991. Radioactive Materials Released from Nuclear Power Plants, Annual Report 1988. NUREG-CR-2907, BNL-NUREG-51581, Vo1. 9, U.S. Nuclear Regulatory Commission, Washington, D.C.

U.S. Bureau of the Census. 1990. Statistical Abstract of the United States: 1990, 110th edition. U.S. Bureau of the Census, Washington, D.C.

U.S. Nuclear Regulatory Commission. 1977. Calculation of Annual Doses to Man from Routine Releases of Reactor Effluents for the Purpose of Evaluating Compliance with 10 CFR Part 50, Appendix I, Revision 1. Regulatory Guide 1.109, Washington, D.C. 
NUREG/CR-2850

PNL-4221

Vol. 10

\section{DISTRIBUTION}

No. of

Copies

\section{OFFSITE}

U.S. Nuclear Regulatory Commission

Division of Technical Information and Document Control

7920 Norfolk Avenue

Bethesda, MD 20014

Steve Baker

ICF Kaiser Engineers

601 Williams

Richland, WA 99352

Cathy Behling

263 01d Limekiln Road

Chalsont, PA 18914

W. E. Bolch

University of Florida

Department of Environmental

Engineering Sciences

AP Black Hall

Gainesville, FL 32611

F. J. Borst

Fort St. Vrain Nuclear Generating Station

16805 Weld County Road 19 1/2

Platteville, CO 80651

K. R. Bräutigam

Kernsforschungszentrum

Karl sruhe

7514 Eggenstein - Leopoldshafen

Karlsruhe

Federal Republic of Germany

H. D. Brenk

Zentralabteilung

Strahlenschultz der

Kernforschungsanlage Jül ich GmbH

Postfach 1913 - 5170 Jülich 1

Federal Republic of Germany
No. of

Copies

J. T. Brennen, M.D.

Radiation Management Corp.

3508 Market Street

Philadelphia, PA 19104

A. J. Colli

Office of Air and Radiation

U.S. Environmental Protection Agency

Washington, DC 20460

R. M. DeNulman

Instituto Nacional De Investigaciones Nucleares

Benjamin Frank lin 161

MEXCIO 11, D. F.

T. Dipierro

Building 475

Brookhaven National Laboratory Upton, NY 11973

P. J. Dostie State of Maine

Department of Human Services

Augusta, ME 04333

D. E. Dunning, Jr.

Evaluation Research Corporation

800 Oak Ridge Turnpike Suite

501

Oak Ridge, TN 37830

K. F. Eckerman

Oak Ridge National Laboratory

P.0. Box X

Oak Ridge, TN 37830

Ecological Sciences Information Center

Oak Ridge National Laboratory

Oak Ridge, TN 37830 
NUREG/CR-2850

PNL-4221

Vol. 10

No. of Copies

T. H. Essig

Office of Nuclear Reactor

Regulation

U.S. Nuclear Regulatory

Commission

Washington, DC 20555

R. J. Feinberg

Knol1s Atomic Power Laboratory

General Electric Company

Box 1072

Schenectady, NY 12301

\section{B. Feldman}

Energy Systems Reserach Group

$120 \mathrm{Milk}$ Street

Boston, MA 02107

J. W. Ferman

Division of Water Quality

Minnesota Pollution Control Agency

1935 West County Road B-2

Roseville, MN 55113

J. F. Greene

Mecklenburg County Department of Environmental Health

1200 Blythe Boulevard

Charlotte, NC 28203

10 R. A. Hartfield

Office of Administration and Resources Management

U.S. Nuclear Regulatory Commission

Washington, DC 20555

J. Hennigan

Division of Radiation Health

Bureau of Environmenal Health

Michigan Department of Public Health

3500 North Logan

Lansing, MI 48906

E. Hewitt

FMC Corporation

Box 41111

Pocatello, ID 83202
No. of

Copies

F. 0. Hoffman

Oak Ridge National Laboratory

P.0. Box X

Oak Ridge, TN 37830

Mark Howard

Congressional Info. Service

4520 EW Highway

Suite 800

Bethesda, MD 20814-3389

L. Huebner

Hazleton Environmental Sciences 1500 Frontage Road

Northbrook, IL 60062

A. P. Hull

Brookhaven National Laboratory Upton, NY 11973

J. Iacovino

Nuclear Safety and Licensing

Westinghouse Electric Corporation

Box 355

Pittsburg, PA 15230

M. L. Joshi

Government of India

Department of Atomic Energy

Rajasthan Atomic Power Project

Nuclear Training Centre

INDIA

B. Kahn

Environmental Resources Center

Georgia Institute of Technology

Atlanta, GA 30332

M. Laraia

C.N.E.N., Disp/Centr

00198 Roma

Viale Regina Margherita, 125

ITALY

L. Lewis

Duke Power Company

422 S. Church Street

Charlotte, NC 28242 
No. of

Copies

S. Mattsson

Lunds Universitet

Radiofysiska Institutionen

Lasarettet, S-221 85 Lund

SWEDEN

H. R. Meyer

Oak Ridge National Laboratory

Oak Ridge, TN 37830

J. Nagy

Building 475

Brookhaven National Laboratory

Upton, NY 11973

S. Morris

Building 475

Brookhaven National Laboratory

Upton, NY 11973

L. Namestek, Ing.

Prager Gasse 11/5

A-3002 Purkersdorf

AUSTRIA

K. K. Narayanan

Bhabha Atomic Research Centre

Bombay - 400085

INDIA

\section{W. Ogg}

PSD CRBRP

P.0. Box U

Oak Ridge, TN 37830

G. V. Oldfield

WPPSS

Mail Drop 1020

P. 0. Box 968

Richland, WA 99352

K. C. Pillai

Health Physics Division

Bhabha Atomic Research Centre

Bombay - 400085

INDIA
No. of

Copies

\author{
C. Pomroy \\ Health and Welfare Canada \\ Health Protection Branch \\ Radiation Protection Bureau \\ ottawa, KlA 1Cl \\ CANADA
}

G. Poretti

ABT. F. MEDIZ. Strahlenphysik

Insel spital

$\mathrm{CH}-3010$ BERN

SWITZERLAND

J. W. Poston

School of Nuclear Engineering

Georgia Institute of Technology

Atlanta, GA 30332

R. R. Roselius

Consumers Power Company

Midland Plant Operations

P.0. Box 1593

Midland, MI 48640

P. Ross

Congressional Information

Service

4520 East West Highway

Washington, DC 20014

V. Sailor

Building 701

Brookhaven National Laboratory

Upton, NY 11973

Lel and Schneider

American Nuclear Insurers

The Exchange, Suite 245

270 Farmington Avenue

Farmington, CT 06032

J. Tadmor

Advisory Committee for Nuclear Safety

Soreq Nuclear Research Centre

Yavne

ISRAEL 
No. of

Copies

T. Thomas

National Institutes of Health

Landow Building, Room 3C16

9000 Rockville Pike

Bethesda, MD 20014

J. Tichler

Building 51

Brookhaven National Laboratory

Upton, NY 11973

A. N. Tshaeche

Mai 1682

General Electric Company

175 Curtner Avenue

San Jose, CA 95125

F. Valerio

Universita Di Genova

Instituto Di Oncologia

16132 Genova

Viale Benedetto XV N.10

ITALY

\section{P. Vasudev \\ Environment Canada \\ Place Vincent Massey \\ 45th Floor \\ Ottawa, K1A 1 C8 \\ CANADA}

G. H. Whipple

3301 Rutland Loop

Tallahassee, FL 32312

E. I. White

9139 S. Bentham Avenue

Sandy, UT 84093

C. A. Willis

Office of Nuclear Reactor

Regulation

U.S. Nuclear Regulatory

Commission

Washington, DC 20555

F. E. Yost

Utility Data Institute, Inc.

2011 I Street, NW, Suite 700

Washington, DC 20006
No of

Copies

\section{$\underline{\text { ONSITE }}$}

2 Westinghouse Hanford Company
H. R. Brager
HO-36
P. R. Prevo
N1 -73

22 Pacific Northwest Laboratory

W. J. Bair K1-50

D. A. Baker (10) K3-54

T. L. Brown

$\mathrm{K} 1-35$

J. R. Johnson

K3 -53

R. L. Moffitt K6-46

P. S. Stansbury K3-53

Health Physics Department Library

Publishing Coordination

Technical Report Files 